\title{
CHINESE UNDERGRADUATE STUDENTS' MOTIVATION PROFILES: \\ IDENTIFICATION AND SIGNIFICANCE
}

BY

JIE GAO

\begin{abstract}
A thesis
submitted to Victoria University of Wellington

in fulfilment of the requirements for the degree of

Doctor of Philosophy
\end{abstract}

Victoria University of Wellington

2019 



\begin{abstract}
This research sought to investigate the motivation of Chinese undergraduate students. It drew on Higgins' (2012) conceptualization of motivation, which defined motivation as involving individuals' simultaneous strivings for value, truth, and control effectiveness. Promotion, prevention, assessment, and locomotion are key general motivation tendencies that measure these three ways of strivings for effectiveness; these motivation tendencies interact to shape motivation effects (Higgins, 2012). This research examined the number and nature of the motivation configurations (i.e., motivation profiles) that mapped the interrelationships among promotion, prevention, assessment, and locomotion for a sample of Chinese undergraduate students. To this end, a mixed methods approach was adopted. First, quantitative data were collected from 886 Chinese undergraduate students at a Chinese university. The quantitative phase identified the motivation profiles and evaluated similarities/differences among the profiles with regard to important motivation factors pertaining to grit, theories of intelligence, critical thinking, effort regulation, and perceptions/evaluations of academic success. Then, the qualitative phase consisted of a multiple-case study of 19 interview participants who were purposefully selected from each of the motivation profiles identified in the quantitative phase.
\end{abstract}

Analyses of quantitative and qualitative data revealed important findings. Specifically, five distinct motivation profiles were determined in the quantitative phase. The first profile (i.e., C1) was characterized by having high/very high and comparable levels of all four motivation orientations. The $\mathrm{C} 2$ profile encompassed very strong prevention and average and comparable levels of the remaining orientations. The C3 profile was characterized by strong/very strong and comparable assessment, locomotion, and promotion, but very weak prevention. The $\mathrm{C} 4$ profile was characterized by having low/very low levels of assessment, locomotion, and promotion and below average levels of prevention. The $\mathrm{C} 5$ profile encompassed below average and comparable levels of assessment, locomotion, promotion, and somewhat stronger prevention. Subsequent 
analyses showed that there were statistically significant differences among the profiles with regard to most of the motivation factors investigated. Some of these differences were of medium to large/very large magnitude. The qualitative phase of this research provided an in-depth understanding of the different strategic approaches that students who have a certain motivation profile reported employing in their learning. The qualitative findings showed that the approaches to learning and learning related tasks/activities that were reported by the interview participants were generally in line with the characteristics of their motivation profiles.

Identifying and discussing Chinese undergraduate students' motivation profiles contribute to understanding how the four general motivation tendencies (i.e., promotion, prevention, assessment, and locomotion) interact and how these interactions shape other motivation factors for this population. Moreover, findings from this research provide salient information regarding the motivation factors that undergird students' academic success. This knowledge can help teachers/educators better understand students' motivation and support productive outcomes in learning settings. In conclusion, this research makes important contributions to the existing motivation literature and educational practices. It also opens up novel directions for future research. Keywords: motivation, strivings for value, truth, and control effectiveness, promotion, prevention, assessment, locomotion, motivation profiles 


\section{Acknowledgements}

This thesis is my beautiful dream come true. The dream would not have been possible without the help and support of many people all the way through my challenging $\mathrm{PhD}$ journey.

Most importantly, I am profoundly indebted to my supervisors. Associate Professor Flaviu Hodis has supported me in each step of the research process and encouraged me to strive for the best. Dr. Carolyn Tait has inspired me by her wit and knowledge. It is under their demanding and inspiring supervision that I have been able to complete my thesis.

My special thanks go to all the participants in this study for their time and contributions. I also owe gratitude to my friends and colleagues in China, who helped me contact potential participants and collect data.

The research for my thesis has been financially supported by Victoria Doctoral Scholarships, Victoria Doctoral Submission Scholarships, and research grants from Victoria University of Wellington. I am grateful for the financial assistance.

Finally, but not least, I would like to extend my deep gratitude to my whole family. Many thanks to my parents for their unconditional love and support. To my husband, Tao, who has walked me through darkness during my doctoral studies. To my lovely daughter, Sunshine, whose beautiful smiles make my day. Thank you all for supporting and encouraging me to pursue my dream. 


\section{Table of Contents}

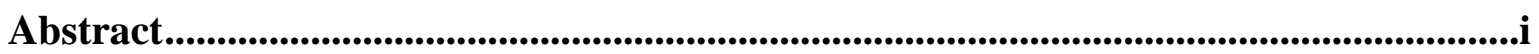

Acknowledgements ......................................................................................................................................... iii

Table of Contents.........................................................................

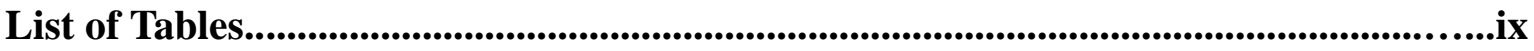

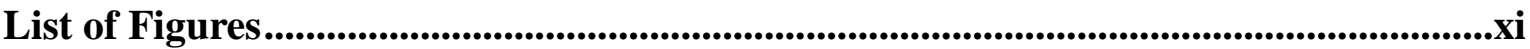

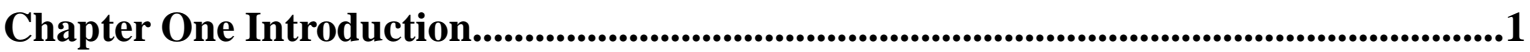

Aim of the Research and Theoretical Framework....................................................... 1

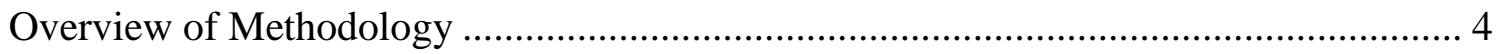

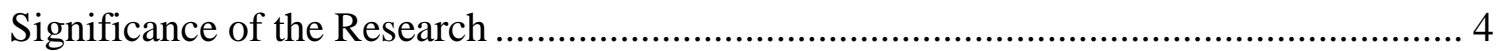

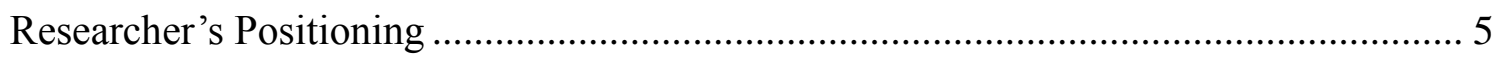

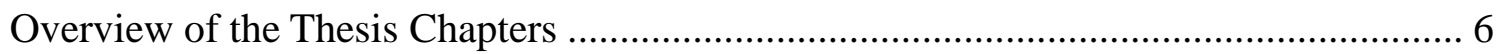

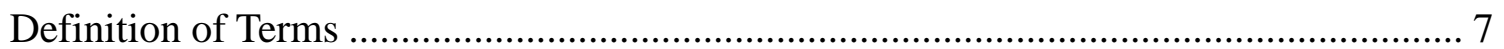

Chapter Two Literature Review ............................................................................................ 11

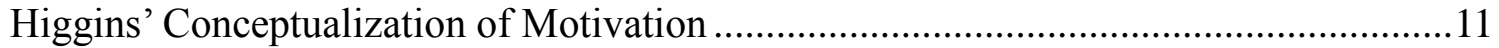

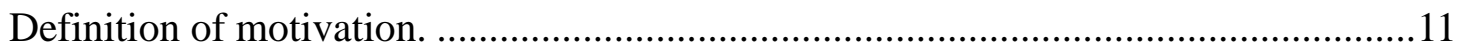

Distinctions among value, truth, and control effectiveness. .................................... 15

Interrelationships among value, truth, and control effectiveness............................... 16

Measuring strivings for value, truth, and control effectiveness................................. 18

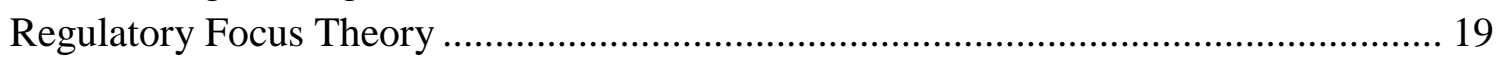

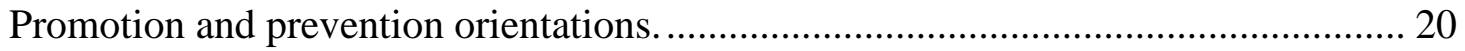

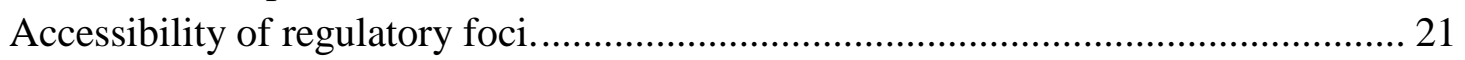

Differences between promotion and prevention orientations. .................................... 22

Relationships between promotion and prevention orientations. ................................. 26

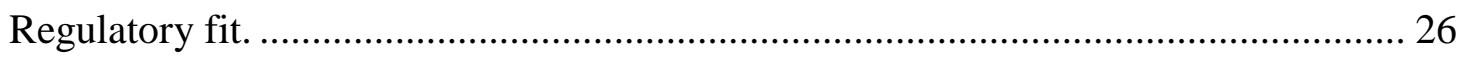

Importance of promotion and prevention in motivation regulation.......................... 27

Distinguishing regulatory focus from approach and avoidance motivations............... 28

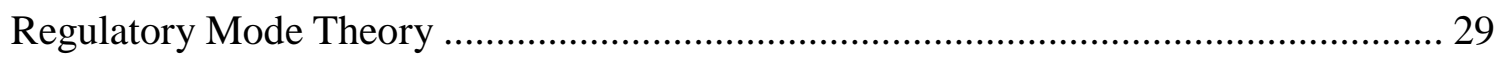

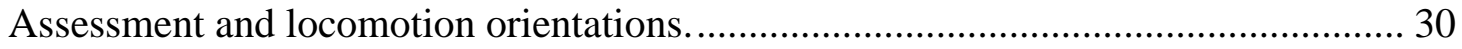

Precursors of assessment and locomotion orientations........................................... 30

Motivational characteristics of assessment and locomotion orientations................... 31

Relationships between assessment and locomotion orientations............................... 33

Interrelationships between Promotion, Prevention, Assessment, and Locomotion

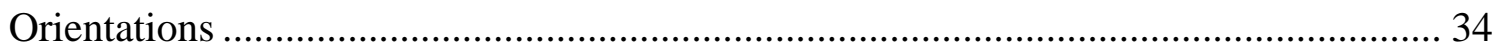

Motivation profiles defined by promotion, prevention, assessment, and locomotion. 36

Relationships between Motivation Orientations and Key Outcomes................................ 38

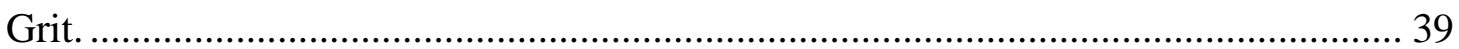

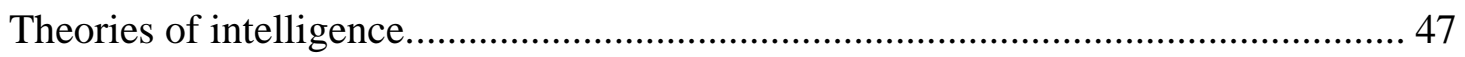

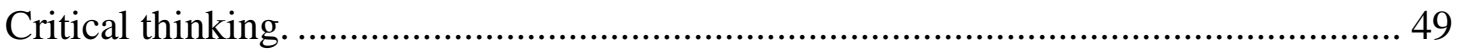

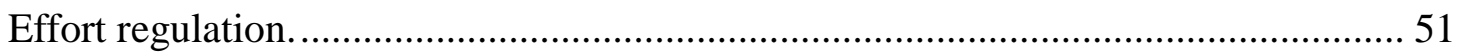

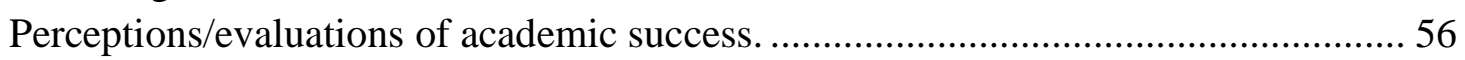




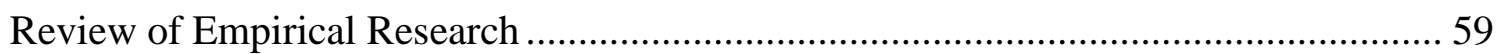

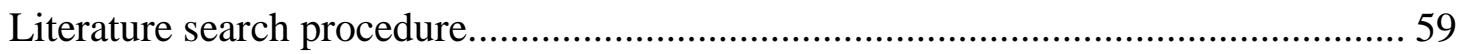

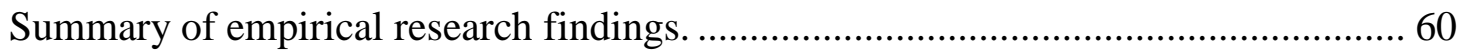

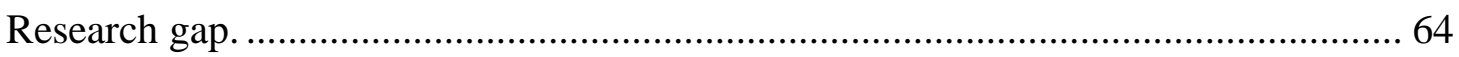

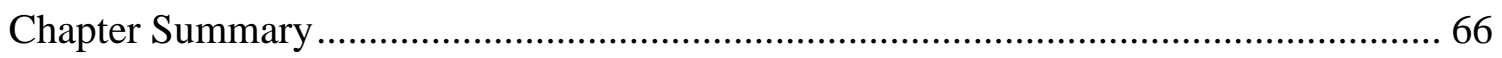

Chapter Three Methodology ...............................................................................................................67

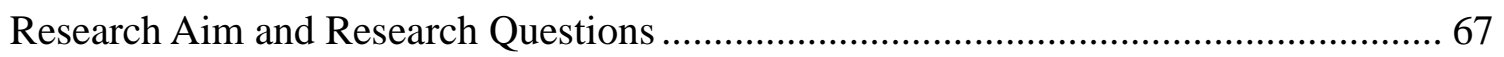

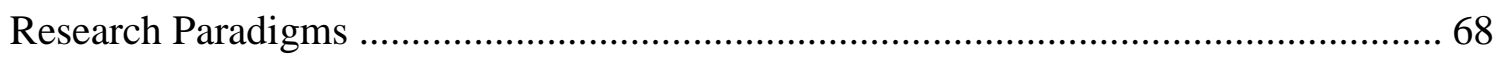

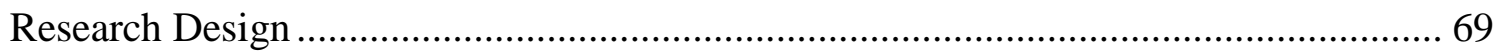

Phase One: Quantitative Data Collection and Analysis ............................................ 72

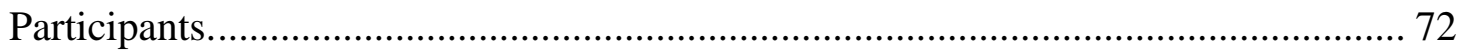

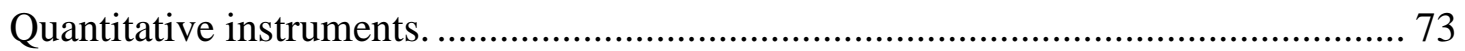

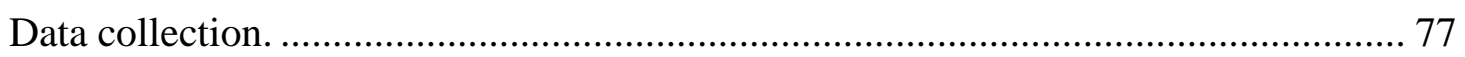

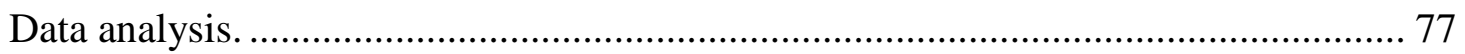

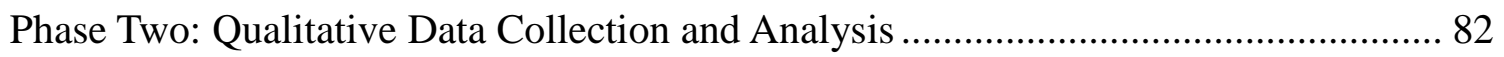

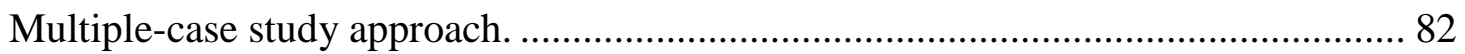

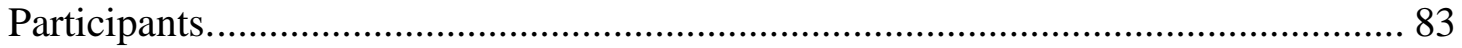

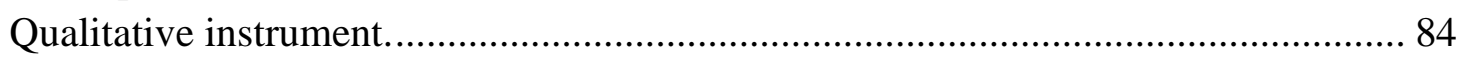

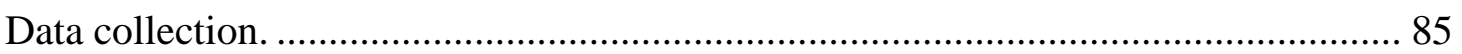

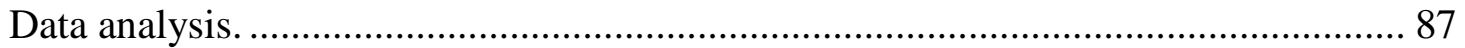

Validity and Trustworthiness of the Research .......................................................... 89

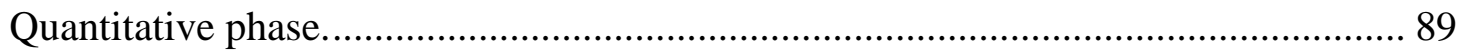

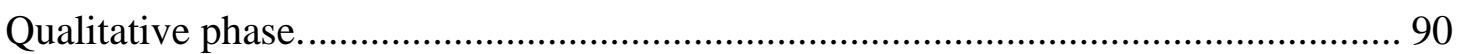

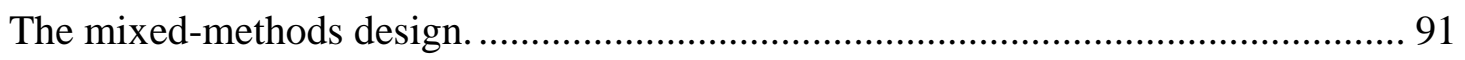

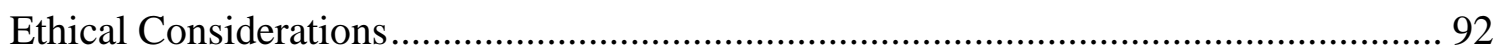

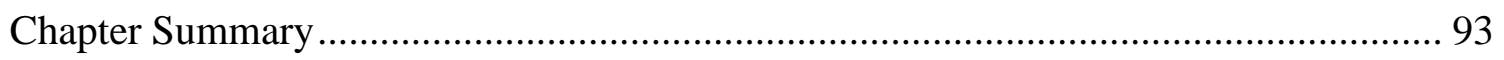

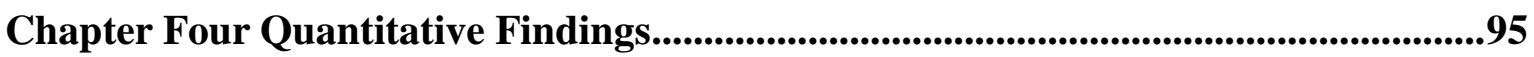

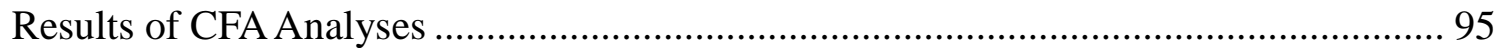

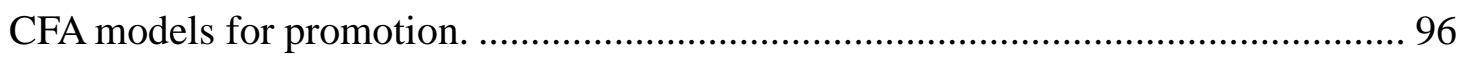

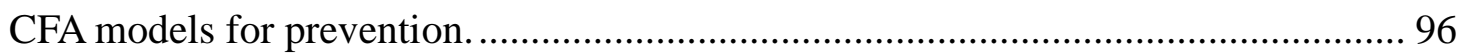

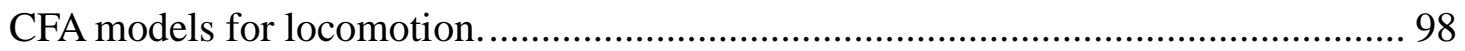

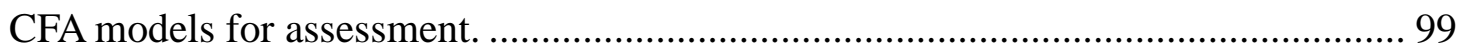

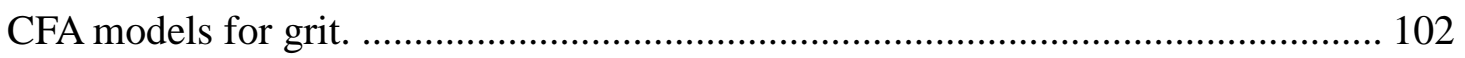

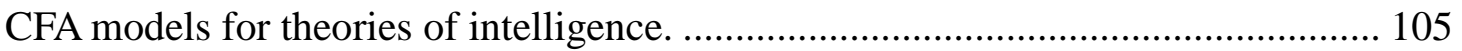

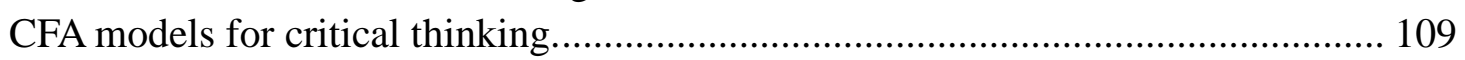

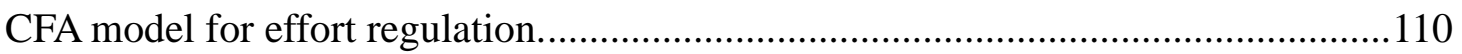

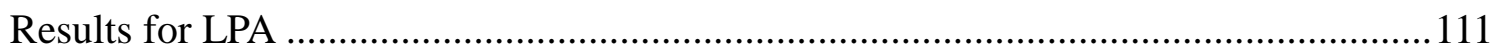

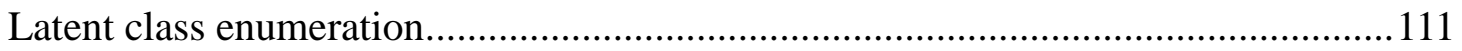

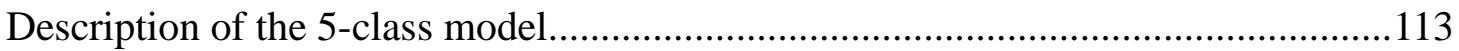

Comparison of Motivation Profiles on Criteria of Interest ....................................... 121

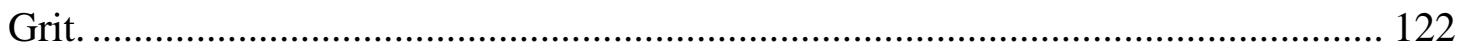

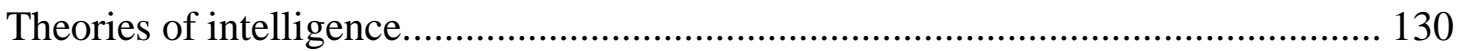

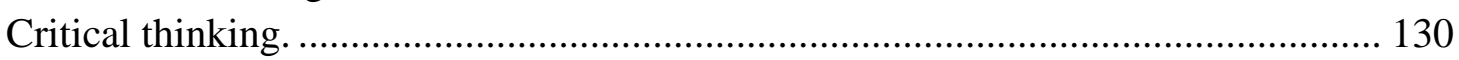




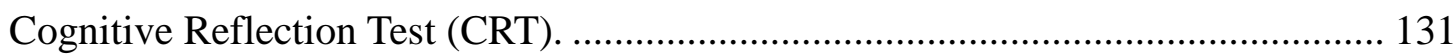

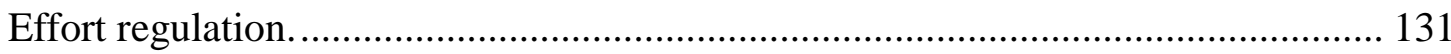

Perceptions/evaluations of academic success. ...................................................... 132

Summary of Characteristics of the Five Motivation Profiles ....................................... 138

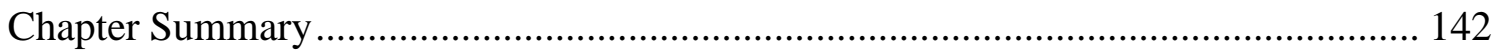

Chapter Five Qualitative Findings .......................................................................................143

Case 1: Participants with the C1 Profile...................................................................... 143

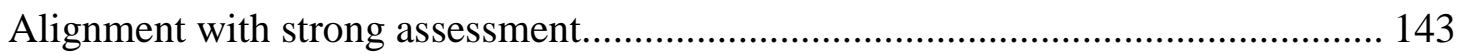

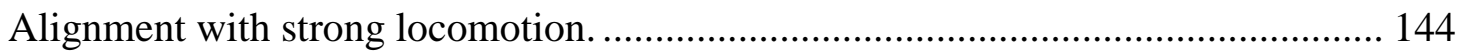

Alignment with dual strong promotion and prevention............................................ 146

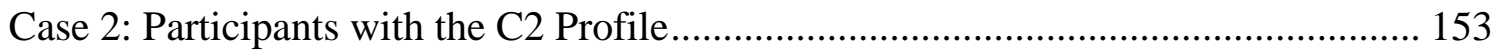

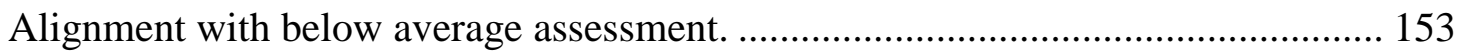

Alignment with below average locomotion............................................................. 154

Alignment with below average promotion. .......................................................... 155

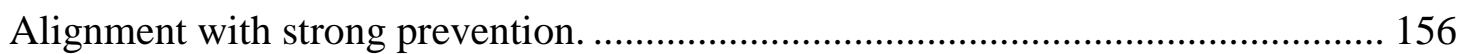

Case 3: Participants with the C3 Profile................................................................... 162

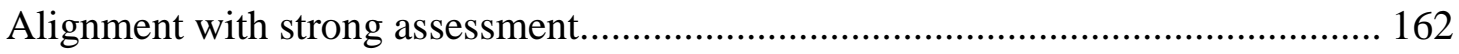

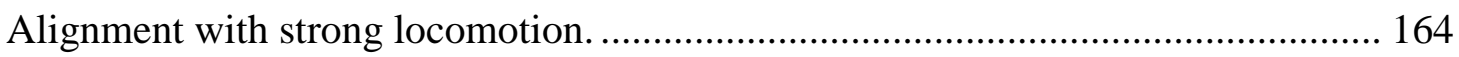

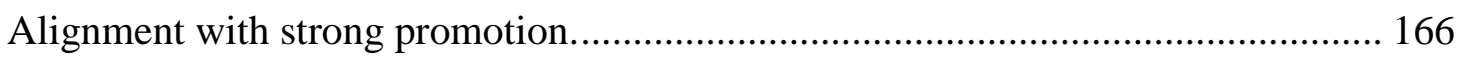

Alignment with weak prevention....................................................................... 168

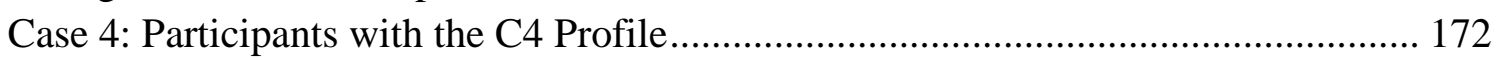

Alignment with weak assessment.................................................................... 172

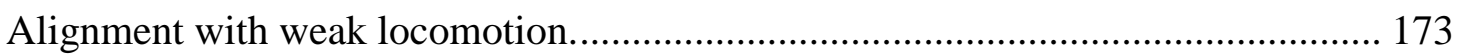

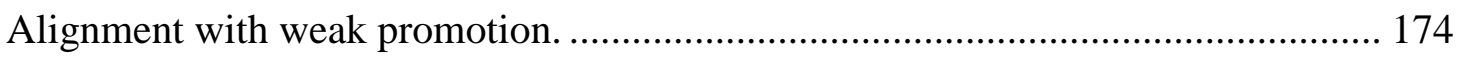

Alignment with below average prevention........................................................ 175

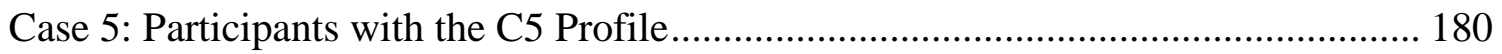

Alignment with below average assessment. ....................................................... 180

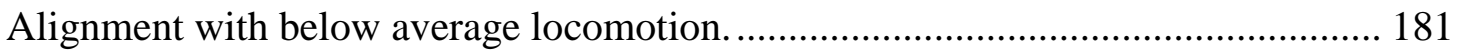

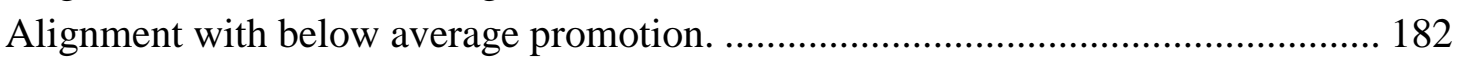

Alignment with below average prevention.......................................................... 183

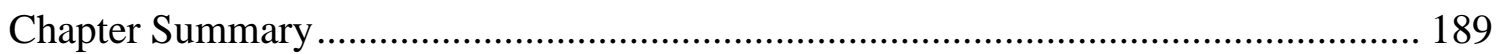

Chapter Six Discussion ................................................................................................................191

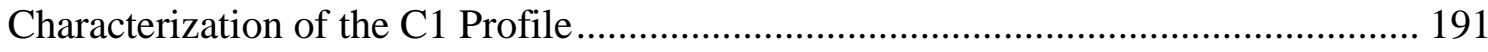

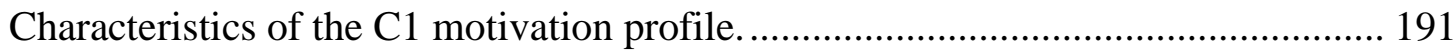

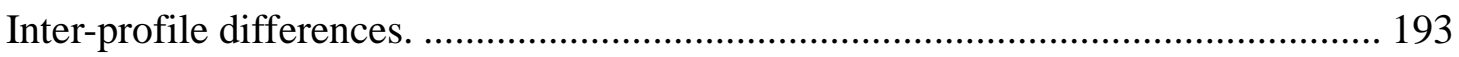

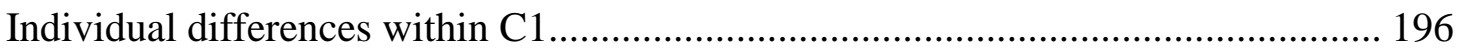

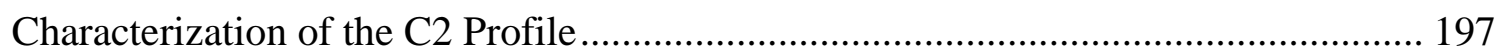

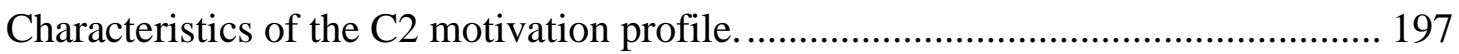

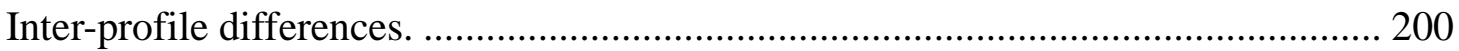

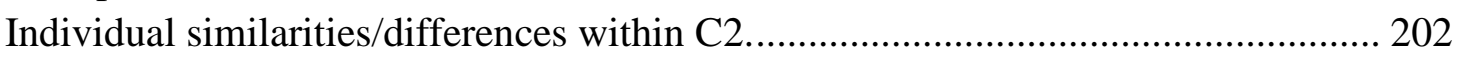

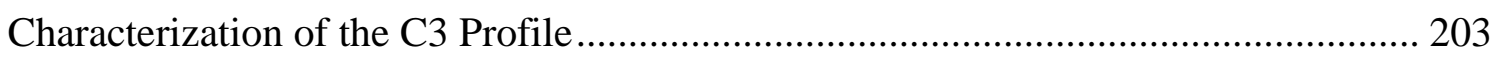

Characteristics of the $\mathrm{C} 3$ motivation profile............................................................ 203

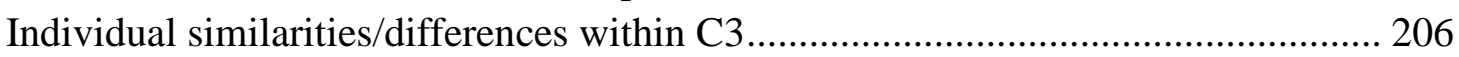




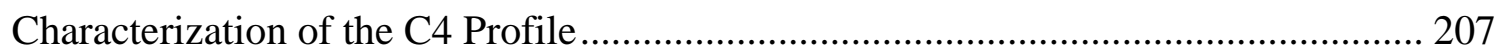

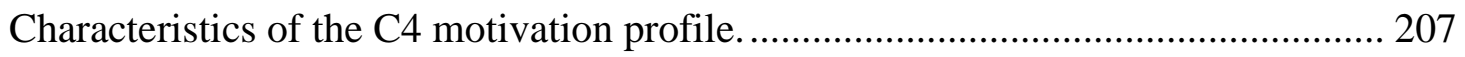

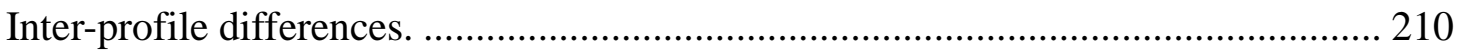

Individual similarities/differences within C4 .................................................... 212

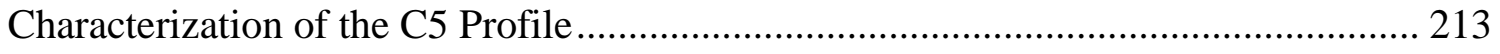

Characteristics of the C5 motivation profile............................................................. 213

Individual similarities/differences within C5 ..................................................... 215

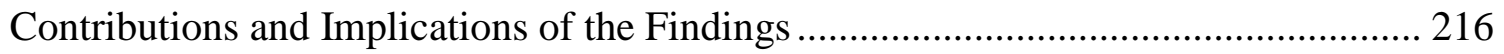

Similarity and variability with regard to motivation profiles.......................217

Supporting Higgins' conceptualization of motivation........................................ 220

Highlighting the important motivational trade-offs among the motivation orientations.

Mapping motivational manifestations associated with different motivation profiles. 228

Motivation profiles and key motivation factors/outcomes. .................................... 231

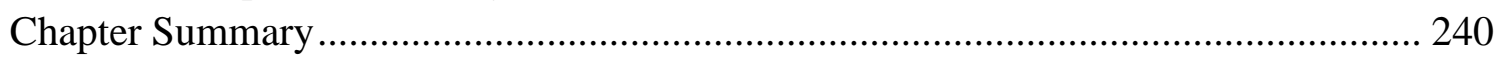

Chapter Seven Conclusion ....................................................................................................................2241

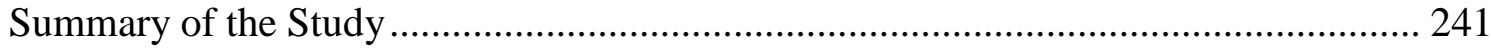

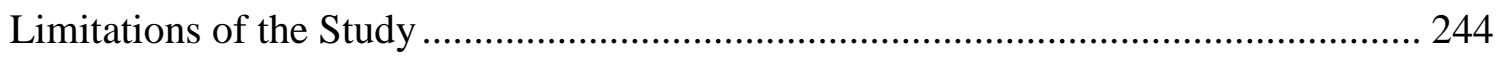

Recommendations for Future Research ............................................................... 245

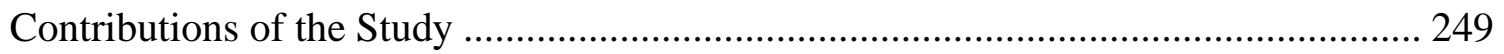

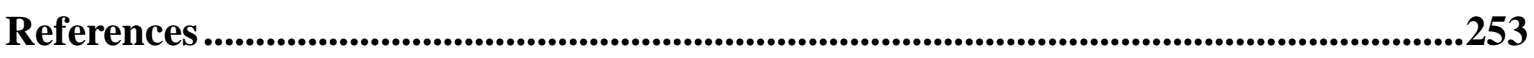

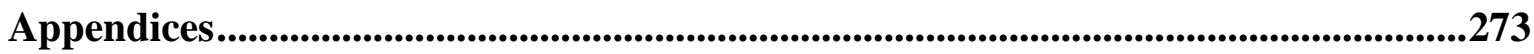

Appendix A: Information Sheet for the Questionnaire Participants............................. 273

Appendix B: Modifications of the Questionnaire Items ............................................ 276

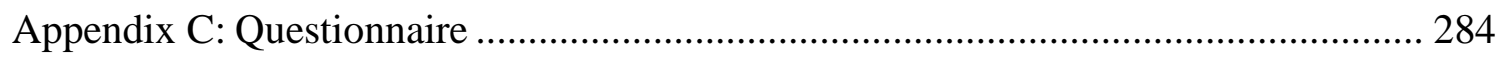

Appendix D: Information Sheet for Interview Participants ........................................ 290

Appendix E: Consent Form for Interview Participants ............................................. 293

Appendix F: Samples of Interview Questions........................................................... 295 


\section{List of Tables}

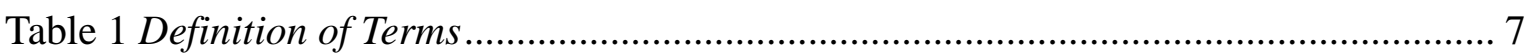

Table 2 Motivational Characteristics of Assessment and Locomotion Orientations............32

Table 3 Demographic Information of Questionnaire Participants ..................................... 73

Table 4 Description of the Measures Used in the Quantitative Phase .................................76

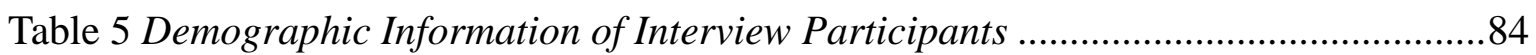

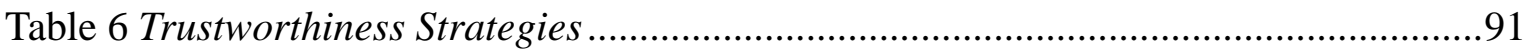

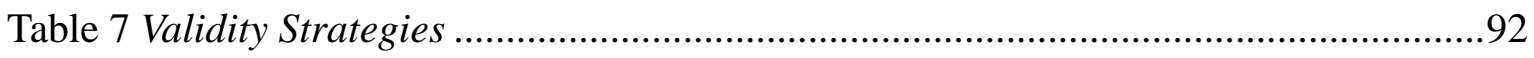

Table 8 Maximum-Likelihood Estimates of Descriptive Statistics and 1-Factor CFA

Standardized Loadings and Standard Errors of Promotion Items ..................................... 96

Table 9 Maximum-Likelihood Estimates of Descriptive Statistics and 1-Factor CFA

Standardized Loadings and Standard Errors of Prevention Items .................................... 97

Table 10 Maximum-Likelihood Estimates of 2-Factor CFA Standardized Loadings and

Standard Errors of Promotion and Prevention Items ...................................................... 98

Table 11 Maximum-Likelihood Estimates of Descriptive Statistics and 1-Factor CFA

Standardized Loadings and Standard Errors of Locomotion Items .................................. 99

Table 12 Maximum-Likelihood Estimates of Descriptive Statistics and 1-Factor CFA

Standardized Loadings and Standard Errors of Assessment Items ................................... 100

Table 13 Maximum-Likelihood Estimates of 2-Factor CFA Standardized Loadings and

Standard Errors of Locomotion and Assessment Items .................................................. 102

Table 14 Maximum-Likelihood Estimates of Descriptive Statistics and 1-Factor

Standardized Loadings and Standard Errors of Consistency of Interest Items ................ 103

Table 15 Maximum-Likelihood Estimates of Descriptive Statistics and 1-Factor CFA

Standardized Loadings and Standard Errors of Perseverance of Effort Items ................. 103

Table 16 Maximum-Likelihood Estimates of Descriptive Statistics and 1-Factor CFA

Standardized Loadings and Standard Errors of Grit Items (i.e., Both Consistency of

Interest and Perseverance of Effort Items) ................................................................... 104

Table 17 Maximum-Likelihood Estimates of 2-Factor CFA Standardized Loadings and

Standard Errors of Consistency of Interest and Perseverance of Effort Items ................. 105

Table 18 Maximum-Likelihood Estimates of Descriptive Statistics and 1-Factor CFA

Standardized Loadings and Standard Errors of Entity Theory of Intelligence Items....... 106

Table 19 Maximum-Likelihood Estimates of Descriptive Statistics and 1-Factor CFA

Standardized Loadings and Standard Errors of Incremental Theory of Intelligence

Items

Table 20 Maximum-Likelihood Estimates of Descriptive Statistics and 1-Factor CFA Standardized Loadings and Standard Errors of the Theory of Intelligence Items (i.e.,

Both Entity and Incremental Theory of Intelligence Items).....

Table 21 Maximum-Likelihood Estimates of 2-Factor CFA Standardized Loadings and

Standard Errors of Entity Theory and Incremental Theory of Intelligence Items

Table 22 Maximum-Likelihood Estimates of Descriptive Statistics and 1-Factor CFA

Standardized Loadings and Standard Errors of Critical Thinking Items ...........................110

Table 23 Maximum-Likelihood Estimates of Descriptive Statistics and 1-Factor CFA 
Standardized Loadings and Standard Errors of Effort Regulation Items. 110

Table 24 Statistical Criteria Associated with Latent Class Enumeration

Table 25 Descriptive Statistics for Classes and Total Samples of the Five-class Model..118

Table 26 Descriptive Statistics and the Results of the Tests of Significance for Differences among Classes with regard to Grit and Theories of Intelligence

Table 27 Descriptive Statistics and the Results of the Tests of Significance for

Differences among Classes with regard to Critical Thinking, Effort Regulation, and

Perceptions/Evaluations of Academic Success

Table 28 Cohen's d Effect Sizes Associated with Mean Differences between Classes with regard to Consistency of Interest

Table 29 Cohen's d Effect Sizes Associated with Mean Differences between Classes with regard to Perseverance of Effort.

Table 30 Cohen's d Effect Sizes Associated with Mean Differences between Classes with regard to Incremental Theory of Intelligence

Table 31 Cohen's d Effect Sizes Associated with Mean Differences between Classes with regard to Entity Theory of Intelligence

Table 32 Cohen's d Effect Sizes Associated with Mean Differences between Classes with regard to Critical Thinking

Table 33 Cohen's d Effect Sizes Associated with Mean Differences between Classes with regard to Cognitive Reflection Test (CRT).....

Table 34 Cohen's d Effect Sizes Associated with Mean Differences between Classes with regard to Effort Regulation

Table 35 Cohen's d Effect Sizes Associated with Mean Differences between Classes with regard to Perceptions/Evaluations of Academic Success

Table 36 Summary of the Four Participants'Data for Each Theme (C1) 151

Table 37 Summary of the Four Participants'Data for Each Theme (C2)

Table 38 Summary of the Three Participants'Data for Each Theme (C3) 170

Table 39 Summary of the Three Participants'Data for Each Theme (C4) 178

Table 40 Summary of the Five Participants' Data for Each Theme (C5)..... 186

Table 41 A Summary of the Characteristics of the Five Motivation Profiles .242 


\section{List of Figures}

Figure 1. Placing Higgins' (2012) conceptualization of motivation in the field of motivation

Figure 2. Schematic representation of Higgins' (2012) model of motivation.

Figure 3. Schematic representation of the conceptualization of promotion and prevention orientations. 25

Figure 4. The mixed methods explanatory sequential design procedures. ........................ 71

Figure 5. The model with five latent classes (i.e., five motivation profiles).....................116

Figure 6. The model with six latent classes (i.e., six motivation profiles)........................117

Figure 7. Average values of consistency of interest across the five classes......................133

Figure 8. Average values of perseverance of effort across the five classes.....................133

Figure 9. Average values of incremental theory of intelligence across the five classes....134

Figure 10. Average values of entity theory of intelligence across the five classes. ..........134

Figure 11. Average values of critical thinking across the five classes.............................135

Figure 12. Average values of Cognitive Reflection Test (CRT) scores across the five classes.

Figure 13. Average values of effort regulation across the five classes.

Figure 14. Average values of perceptions/evaluations of academic success across the five classes.

Figure 15. Summary of class-specific mean values of six criteria 


\section{Chapter One Introduction}

Higgins (2012) defined motivation as wanting to be effective in goal pursuits with regard to achieving valued outcomes, finding the truth about oneself and her/his experiences, and having control over all aspects of goal pursuits. Motivation is a critical factor that influences students' engagement with school and their performance in learning settings (Eccles \& Wigfield, 2002; Molden \& Rosenzweig, 2016; Wigfield, Muenks, \& Rosenzweig, 2015). This research investigates the motivation of Chinese undergraduate students and interindividual differences in attendant motivation effects pertaining to grit, theories of intelligence, critical thinking, effort regulation, and perceptions/evaluations of academic success. A brief definition of the key terms used in this study is provided in Table 1 at the end of this chapter.

In this chapter, I introduce the aim, the theoretical framework, and research questions of the study. Moreover, I provide a brief overview of the methodology adopted in the study. I also highlight the significance of the research and the researcher's positioning and describe the structure of the thesis.

\section{Aim of the Research and Theoretical Framework}

Motivation has been of great interest to researchers and practitioners in psychology, education, and other disciplines (Eccles \& Wigfield, 2002; Schunk, Pintrich, \& Meece, 2008). Molden and Rosenzweig (2016) argued that

...the type of motivation that guides students' academic pursuits can substantially alter the strategies they use for these pursuits, how they process information about the progress they are making, and how they respond to their short-term successes or failures, all of which influence their long-term performance (p. 477).

Empirical findings provide evidence of a strong relationship between motivation and key aspects of learning. For example, motivated learners tend to show greater interest in learning activities, are willing to expend more effort in learning, persist longer at tasks, and 
employ appropriate learning strategies (Eccles \& Wigfield, 2002; Wigfield et al., 2015). Motivational beliefs (e.g., utility value of learning) also influence students' choice of academic activities such as selecting a course or major (Musu-Gillette, Wigfield, Harring, \& Eccles, 2015; Simpkins, Davis-Kean, \& Eccles, 2006).

Moreover, motivation has been identified as an important contributor to academic achievement (Meyer, McClure, Walkey, Weir, \& McKenzie, 2009; Wigfield \& Cambria, 2010; Wigfield et al., 2015). For instance, students' patterns of motivation are predictive of different achievement trajectories: students who strive for the highest marks in an academic assessment are likely to attain acceptable/good grades, whereas students who set low goals for achievement and aim to attain the lowest passing marks have high probabilities of academic failure (Hodis, Meyer, McClure, Weir, \& Walkey, 2011). Notably, a lack of motivation is not only associated with decreased interest in learning and disengagement from school, but also with underachievement and school dropout (Janosz, Archambault, Morizot, \& Pagani, 2008; Scheel, Madabhushi, \& Backhaus, 2009).

Given the importance of motivation in students' learning, this research seeks to provide a comprehensive understanding of Chinese undergraduate students' motivation and attendant motivation effects. It builds on a motivation framework proposed by Higgins (2012). This framework captures the multidimensional nature of motivation. Specifically, Higgins (2012) posited that being motivated involves strivings for being effective with regard to (a) attaining valued outcomes and avoiding undesired ones (i.e., having value effectiveness), (b) understanding what is real and correct about one's experiences (i.e., having truth effectiveness), and (c) exerting influence with regard to the means and strategies one employs to attain a given goal (i.e., having control effectiveness; Higgins, 2012). Four general motivation tendencies/orientations can be used to capture the three types of effectiveness strivings. Specifically, promotion and prevention gauge value effectiveness; assessment measures truth effectiveness; locomotion pertains to control effectiveness (Higgins, 2012; Hodis, 2018a, 2018b; Hodis, Hattie, \& Hodis, 2017). These motivation orientations work together to influence behaviors, cognitive states, and emotional reactions to success and failure (Higgins, 2012). The roles and functioning of each motivation orientation depend on the strengths of the other orientations; therefore, they interact to shape motivation effects 
(Higgins, 2012; Hodis, 2018a). The interrelationships among promotion, prevention, assessment, and locomotion can be examined by means of motivation profiles, which summarize both the absolute and relative strengths of each of the four orientations (Higgins, 2012; Hodis, 2018a; Hodis et al., 2017; Pierro, Chernikova, Lo Destro, Higgins, \& Kruglanski, 2018).

This research has three important goals. The first is to identify the motivation profiles that map the interrelationships among the pivotal motivation tendencies (i.e., promotion, prevention, assessment, and locomotion that gauge value, truth, and control effectiveness) in a sample of Chinese undergraduate students. The second is to evaluate whether there are significant similarities/differences among the profiles uncovered with regard to key motivation factors that influence students' learning and academic performance. The third is to examine the extent to which students' reported approaches to learning are consistent with the characteristics of their motivation profiles. The following research questions guide this study:

1. How many motivation configurations (i.e., motivation profiles that measure the absolute and relative magnitudes of promotion, prevention, assessment, and locomotion orientations) account parsimoniously for variability in the interrelationships among these key motivation orientations in a sample of mainland Chinese undergraduate students?

2. Are there differences among the motivation profiles identified in this research with regard to students' levels of grit?

3. Are there differences among the motivation profiles identified in this research with regard to students' theories of intelligence?

4. Are there differences among the motivation profiles identified in this research with regard to students' use of critical thinking?

5. Are there differences among the motivation profiles identified in this research with regard to students' effort regulation?

6. Are there differences among the motivation profiles identified in this research with regard to students' perceptions/evaluations of academic success?

7. How do approaches to study reported by the participants align with their motivation profiles? 


\section{Overview of Methodology}

This research used an explanatory mixed methods design. The first six research questions were investigated using the quantitative methods; the last question was explored using qualitative methods. In the quantitative phase of the study, paper questionnaires were used to collect data from undergraduate students in a Chinese university. The quantitative analyses (a) enabled the identification of the distinct motivation profiles undergirding students' different types of effectiveness strivings and (b) helped highlight similarities/differences among profiles with regard to key motivation factors (i.e., grit, theories of intelligence, critical thinking, effort regulation, and perceptions/evaluations of academic success). Qualitative data and their analysis shed additional light on the quantitative findings by exploring participants' personal experiences and subjective views with regard to their strategic approaches to learning.

\section{Significance of the Research}

This study contributes to new and important knowledge of motivation. First, identifying Chinese undergraduate students' motivation profiles and exploring betweenprofile differences in key motivation factors could expand the literature of motivation in general. The concept of motivation profiles (i.e., patterns of associations among promotion, prevention, assessment, and locomotion) has just begun to be investigated (Higgins, 2012; Hodis, 2018a; Pierro et al., 2018). Moreover, current research has yet to examine whether motivation profiles uncovered in western cultures (e.g., New Zealand; Harring \& Hodis, 2016; Hodis et al., 2017) are similar to, or different from, motivation profiles found in eastern cultures (e.g., China). Second, findings regarding between-profile differences with respect to multiple outcomes investigated in the study could advance understanding of how distinct combinations of absolute and relative strengths of promotion, prevention, assessment, and locomotion account for inter-individual differences in important motivation factors.

Third, findings from this study could extend knowledge of Chinese undergraduate students' motivation. Identifying and discussing Chinese undergraduate students' motivation profiles will shed fresh light on how promotion, prevention, assessment, and locomotion 
interact to shape motivation manifestations/factors for members of this population. Finally, it is anticipated that the findings of the study will provide potentially important information regarding the motivation factors that support academic success of Chinese undergraduate students. This information could contribute to teaching and learning in Chinese universities by helping teachers to better understand their students' motivation and assisting them in identifying pedagogical strategies that optimally match their motivation profiles.

\section{Researcher's Positioning}

The researcher's personal experiences may shape her research topic and interpretation (Creswell, 2014). I studied as an English major for seven years at a Chinese university and obtained a Bachelor's degree and a Master's degree. After graduation, I taught English at the undergraduate level for ten years. The experience of studying and teaching at Chinese universities has offered me opportunities to observe students' engagement with learningrelated tasks/activities and enabled me to communicate with students about their motivation and learning experiences. Therefore, I can position myself as an insider who is familiar with learning and teaching contexts at Chinese universities. My knowledge of teaching and learning processes in the target university may also complement my understanding of theoretical aspects and support my interpretation of the data.

My professional experiences aroused my research interest in students' motivation. During years of teaching, I noticed that a portion of Chinese university students seemed to simply want to pass examinations and obtain a degree; they did not engage strongly in learning. In contrast, some other students appeared to have strong motivation and worked hard to achieve their learning goals. As a teacher, I wanted to know what motivated my students to learn. This prompted me to explore the extent to which students' general motivation tendencies related to motivation factors that influence learning and academic performance. As a teacher and researcher, I could use this knowledge to improve my teaching and support students in their academic learning. 


\section{Overview of the Thesis Chapters}

There are seven chapters in the thesis. In Chapter 1, I outline the aim, the research questions, and the significance of the study. In addition, I address how my educational and professional backgrounds have shaped my positioning in conducting the research. Moreover, I present brief overviews of the theoretical framework, the methodology, and the structure of the thesis.

In Chapter 2, I review the literature in relation to Higgins' (2012) conceptualization of motivation, the regulatory focus theory (Higgins, 1997, 2012), and the regulatory mode theory (Kruglanski et al., 2000). I also present the potential links between the four pivotal motivation tendencies (i.e., promotion, prevention, assessment, and locomotion) and other motivation factors that influence students' learning and/or performance in learning settings (i.e., grit, theories of intelligence, critical thinking, effort regulation, and perceptions/evaluations of academic success). Finally, in Chapter 2, I review the empirical studies that examined the regulatory focus/mode orientations of Chinese/Asian learners and identify the gaps that highlight the need for further research that focuses on Chinese learners.

In Chapter 3, I describe the methodology used in this research. Specifically, I introduce the research paradigms that underpin the mixed-methods design of this study and provide a brief overview of the research design. Then, I delineate the details of the quantitative and qualitative phases separately with regard to participant selection, instruments, methods, and procedures of data collection and analysis. Lastly, I address validity and trustworthiness issues and ethical considerations.

I present the quantitative findings in Chapter 4. Specifically, I report the number and nature of the motivation profiles uncovered in the quantitative phase. In addition, I compare and evaluate similarities and differences among these profiles with regard to important motivation factors pertaining to grit, theories of intelligence, critical thinking, effort regulation, and perceptions/evaluations of academic success.

In Chapter 5, I report the qualitative findings. For each profile, I present approaches to learning reported by interview participants selected from a particular profile and evaluate the extent to which these reported approaches aligned to characteristics of a given motivation 
profile.

In Chapter 6, I integrate the findings from the quantitative and qualitative phases to provide in-depth insights into the characteristics of each of the five motivation profiles identified. In addition, I discuss the key contributions of the study and the important theoretical and practical implications of the findings.

In the final chapter, I provide a summary of the study and reflect on its limitations. Moreover, I delineate possible directions for future research.

\section{Definition of Terms}

A brief definition of the key terms in the study is presented in Table 1. The literature related to these terms will be reviewed in the next chapter.

Table 1

Definition of Terms

Key term Definition

Motivation Motivation refers to having preferences that direct choices aimed at being effective in life pursuits (Higgins, 2012).

Value effectiveness Being effective at attaining valued outcomes and avoiding undesired ones (Higgins, 2012).

Truth effectiveness Being effective at understanding what is real and correct about oneself and one's experiences (Higgins, 2012).

Control Being effective at exerting influence over means and strategies effectiveness used in goal pursuits (Higgins, 2012)

Promotion A promotion focus is a general motivation tendency that pertains to value effectiveness (Higgins, 2012). High promotion is associated with paying close attention to presence or absence of positive outcomes and being motivated to achieve ideals and aspirations (Higgins, 1997, 2012).

Prevention A prevention focus is a general motivation tendency that pertains to value effectiveness (Higgins, 2012). High prevention is associated with being sensitive to presence or absence of negative outcomes and striving to maintain security and fulfil duties and responsibilities (Higgins, 1997, 2012). 
Key term Definition

Assessment Assessment is a general motivation tendency that measures strivings for truth-effectiveness (Higgins, 2012). Assessment "constitutes the comparative aspect of self-regulation concerned with critically evaluating entities or states, such as goals or means, in relation to alternatives in order to judge relative quality" (Kruglanski et al., 2000, p. 794).

Locomotion

Locomotion is a general motivation tendency that reflects strivings for control effectiveness (Higgins, 2012). Locomotion "constitutes the aspect of self-regulation concerned with movement from state to state and with committing the psychological resources that will initiate and maintain goalrelated movement in a straightforward and direct manner, without undue distraction or delays" (Kruglanski et al., 2000, p. 794).

Motivation profiles Motivation profiles reflect different patterns of interrelationships among promotion, prevention, assessment, and locomotion. A motivation profile measures the strength of each tendency, and, thus, provides information on both the absolute and relative strengths of the four tendencies. For example, an individual could have a motivation profile that is characterized by high levels of promotion, high levels of assessment, high levels of locomotion, and low levels of prevention (Hodis, 2018a).

Grit

Grit reflects an individual's "perseverance and passion for longterm goals" (Duckworth, Peterson, Matthews, \& Kelly, 2007, p. 1087). This construct consists of two facets: perseverance of effort and consistency of interest (Duckworth et al., 2007; Duckworth \& Quinn, 2009). The former refers to the tendency to work hard despite obstacles, challenges, and failures; the latter describes the tendency to focus on the same long-term goal or interest for a long period of time (e.g., months and years).

Theories of Theories of intelligence refer to people's different beliefs about intelligence the nature of their intelligence. Specifically, an entity theory of intelligence refers to individuals' beliefs that intelligence is a fixed trait that cannot be changed. In contrast, an incremental theory of intelligence refers to individuals' beliefs that intelligence can be changed and developed through effort (Dweck, 1999). 


\begin{tabular}{|c|c|}
\hline Key term & Definition \\
\hline Critical thinking & $\begin{array}{l}\text { Critical thinking is a cognitive process that involves deliberate } \\
\text { and effortful reflection, analysis and evaluation of extant } \\
\text { evidence such as observation, experience, and communications } \\
\text { with others (Byrnes \& Dunbar, 2014). }\end{array}$ \\
\hline Effort regulation & $\begin{array}{l}\text { Effort regulation refers to individuals' ability to devote effort and } \\
\text { maintain attention in the face of challenging or uninteresting } \\
\text { tasks, and other distractions in learning environments (Pintrich \& } \\
\text { De Groot, 1990; Pintrich, Smith, Garcia, \& McKeachie, 1991). }\end{array}$ \\
\hline $\begin{array}{l}\text { Perceptions/ } \\
\text { evaluations of } \\
\text { academic success }\end{array}$ & $\begin{array}{l}\text { Perceptions/evaluations of academic success reflect students' self- } \\
\text { reported evaluations of their previous academic achievements at } \\
\text { university. }\end{array}$ \\
\hline $\begin{array}{l}\text { Motivation } \\
\text { regulation }\end{array}$ & $\begin{array}{l}\text { Motivation regulation is the process by which people monitor and } \\
\text { control the level (i.e., low vs. high) and the type (e.g., promotion } \\
\text { vs. prevention) of their motivation to achieve valued goals (Miele } \\
\& \text { Scholer, 2018). }\end{array}$ \\
\hline Self-regulation & $\begin{array}{l}\text { "Self-regulation is a process in which people organize and } \\
\text { manage their capacities - that is, their thoughts (e.g., competence } \\
\text { beliefs), emotions (e.g., interest), behaviors (e.g., engagement } \\
\text { with learning activities), and social-contextual surroundings (e.g., } \\
\text { select a quiet, comfortable place to study) - in the service of } \\
\text { attaining some desired future state" (Reeve, Ryan, Deci, \& Jang, } \\
\text { 2008, p. 223). }\end{array}$ \\
\hline
\end{tabular}

Note. In the subsequent chapters, the terms "tendency" and "orientation" are used interchangeably when they refer to promotion, prevention, assessment, and locomotion. 


\section{Chapter Two Literature Review}

Within this chapter the motivation literature relevant to the study is reviewed. It begins by introducing Higgins' (2012) theorizing of strivings for value, truth, and control effectiveness in goal pursuits. Then, promotion, prevention, assessment, and locomotion, the key motivation tendencies that measure these effectiveness strivings, are described. Furthermore, the interrelationships among these four motivation orientations and their associations with other motivation factors (i.e., grit, theories of intelligence, critical thinking, effort regulation, and perceptions/evaluations of academic success) are discussed. Finally, empirical research on Chinese and other Asian learners' motivation orientations is reviewed and research gaps in the existing literature are also discussed.

\section{Higgins' Conceptualization of Motivation}

Definition of motivation. As a complex psychological phenomenon, motivation has been defined in a variety of ways; in addition, much disagreement exists about the precise nature of motivation (Schunk et al., 2008). For example, motivation has been traditionally regarded as approaching pleasure and avoiding pain (Higgins, 1997, 2012). Higgins (2012) defined motivation as having preferences that direct choices aimed at being effective in life pursuits. This conceptualization of motivation as striving for effectiveness encompasses all essential aspects of goal pursuit (Franks \& Higgins, 2012; Higgins \& Scholer, 2015). Specifically, motivated individuals strive to be effective with regard to value, truth, and control. According to Higgins (2012), value effectiveness describes the motivation to attain the desired outcomes, namely, having "success in ending with benefits versus costs, pleasure versus pain, biological needs satisfied versus unsatisfied”' (Higgins, 2012, p. 49). Individuals' strivings for truth effectiveness involve trying to find and establish what is real or correct; they do so by exploring and attempting to comprehend what happens and why things happen and by creating a shared reality with others (Higgins, 2012, 2013). The concept of shared reality refers to sharing with others inner states, such as beliefs, feelings, and attitudes about the world (Higgins, 2012). By creating a shared reality with others, people could transform their subjective experience to reliable and valid objective truth (Higgins, 2012). Notably, 
individuals are motivated not only to be accurate, but also to experience themselves as "being successful in having understandings, beliefs and knowledge which are the truth" (Higgins, 2013, p. 100).

The third component of Higgins' conceptualization of motivation, namely, control effectiveness, refers to being successful at managing the required procedures, competencies, and resources needed to make positive things happen and negative things not happen (Higgins, 2012). Generally, individuals want to exert influence over their actions so as to bring about desirable outcomes; nevertheless, in some cases they are motivated to strive for control effectiveness for its own sake.

Higgins' conceptualization of motivation captures the multidimensional nature of motivation. Value effectiveness, that is, striving to have desired results, is critical to human development and has received much attention in the motivation literature (e.g., drive theories, the hedonic principle, and goal theories; Higgins, 2012, 2013; Higgins \& Scholer, 2015). Nevertheless, the other two fundamental strivings (i.e., for truth and control effectiveness) also play key roles in motivation and self-regulation. These two dimensions are independent of and distinct from striving to achieve valued outcomes (Franks \& Higgins, 2012; Higgins, 2012). Without truth effectiveness, individuals will feel confused and doubtful; without control effectiveness, individuals will feel helpless and incompetent (Higgins, 2012). Clearly, individuals are motivated to pursue valued outcomes. At the same time, they strive to establish the truth concerning themselves and their experiences (e.g., making accurate selfjudgments); these strivings for truth effectiveness influence how they compare and evaluate goals and means to achieve goals (Higgins, 2012; Molden \& Higgins, 2012). For instance, people who have strong truth concerns tend to spend a lot of time evaluating the benefits and costs of all options and pay more attention to accuracy than speed (Higgins, Kruglanski, \& Pierro, 2003). Individuals also attempt to exert control over their actions (e.g., they prefer to use certain means to attain goals). These strategic preferences could affect how individuals engage in goal pursuits (e.g., preference for information processing strategies that prioritize speed vs. accuracy; Molden, 2012). Therefore, it is important to take into consideration all these three ways of being effective when analyzing motivation and motivation effects.

Higgins' model of motivation shares conceptual overlaps with some major motivation 
theories (see Figure 1). A strand of motivation literature has focused on goals/values people desire or intend to achieve when they engage in different activities. Goals are cognitive representations of positive outcomes that individuals are committed to attain (Mann, de Ridder, \& Fujita, 2013). Achievement goals are specific goals people want to attain in their engagement of achievement-related activities.

The achievement goal theory differentiates two types of achievement goals, that is, mastery and performance goals (Elliott \& Dweck, 1988; Grant \& Dweck, 2003, Senko, Hulleman, \& Harackiewicz, 2011). People who adopt mastery goals are motivated to acquire or develop their competence. By contrast, people who pursue performance goals are motivated by the desire to display their competence or outperform the others (Senko et al., 2011). Apart from being motivated to achieve goals, people are driven by perceived value for doing a task (Eccles \& Wigfield, 2002). They are inclined to engage in tasks that they value positively and avoid engaging in tasks that they value negatively. The expectancy-value theory defines three important types of task value (i.e., importance/value of doing a given task; Eccles \& Wigfield, 2002; Wigfield, Tonks, \& Klauda, 2009). Specifically, attainment value refers to the personal importance individuals attach to a task because engagement in this task is consistent to who one truly is (i.e., one's self-image; Eccles \& Wigfield, 2002; Miele \& Scholer, 2018). For example, a student who identifies herself as an athlete will have high attainment value for doing a major in sports because doing well in this major might affirm her self-identity. Utility value pertains to usefulness of a task or how a task is related to one's current and future goals. People tend to engage in a task when doing well on this task is useful to attain some long-term goal(s) that they value. For instance, a student will have a high level of utility value with regard to doing well in high school mathematics courses because studying mathematics will help her undertake university studies and explore more advanced areas such as economics. Intrinsic value is the enjoyment an individual obtains from engaging in a task (Eccles \& Wigfield, 2002). Individuals have strong intrinsic value for a task when they are interested in the task and enjoy doing it. In sum, these motivation theories examine people's strivings for valued goals or outcomes. According to these theories, individuals have preferences regarding the types of outcomes they try to attain (e.g., mastery vs. performance goals; different types of task values). These preferences are conceptually 
parallel to strivings for value effectiveness in Higgins' (2012) conceptualization of motivation. That is, people are motivated to attain positive/valued outcomes and avoid negative/undesired outcomes.

The attribution theory explores how people perceive causes of success and/or failure (Eccles \& Wigfield, 2002). In achievement contexts, students may attribute their achievement outcomes to causes such as ability, effort, task difficulty, and luck (Weiner, 1985, 2010). The attribution theory is based on the fundamental concept that people seek to understand their environment through explaining causes for their and other people's behaviors (Weiner, 1979). This concept overlaps with strivings for truth effectiveness in Higgins' (2012) model of motivation; that is, people want to establish what is real and to make sense of their circumstances. Individuals' causal attributions for achievement outcomes may influence their strivings for truth effectiveness. For example, if a student attributes her achievement failure to her lack of ability, she may tend to see herself as being incompetent in learning and believe that she is unlikely to achieve academic success in the future.

The self-determination theory proposes that people have basic psychological needs for autonomy, competence, and relatedness; these three basic psychological needs are essential to human well-being (Ryan \& Deci, 2017). Particularly relevant to this thesis, competence reflects individuals' need to feel effective at interacting with their environment and in important aspects of their lives (e.g., learning at school; Ryan \& Deci, 2017). They want to feel able to take on and master challenges (Ryan \& Moller, 2017). This need corresponds to strivings for control effectiveness in Higgins' (2012) theorizing of motivation; that is, being successful at exerting control over goal pursuit to make positive things happen and negative things not happen.

As I discussed previously, there are conceptual overlaps between Higgins' (2012) conceptualization of motivation and major motivation theories. However, what makes Higgins' motivation theorizing unique is that Higgins argued that value, truth, and control effectiveness are all key dimensions of motivation. Hence, in his view, being motivated encompasses strivings to be effective with regard to achieving valued outcomes and avoiding undesirable ones (i.e., having value effectiveness), finding out the truth about oneself and one's experiences (i.e., attaining truth effectiveness), and having control over one's goal 
pursuits (i.e., possessing control effectiveness; Higgins, 2012). More importantly, the core of Higgins' conceptualization of motivation is that these three types of effectiveness strivings interact with one another to shape motivation and motivation effects. In contrast, the other motivation theories focus only on one dimension of motivation. Specifically, as noted above, achievement goal theory and expectancy-value theory stress that people are motivated to attain valued goals/outcomes (i.e., having value effectiveness); attribution theory highlights people's strivings to understand themselves and their environments by causal attributions of success/failure (i.e., having truth effectiveness); self-determination theory proposes that people seek to feel competent in their environments (i.e., having control effectiveness).

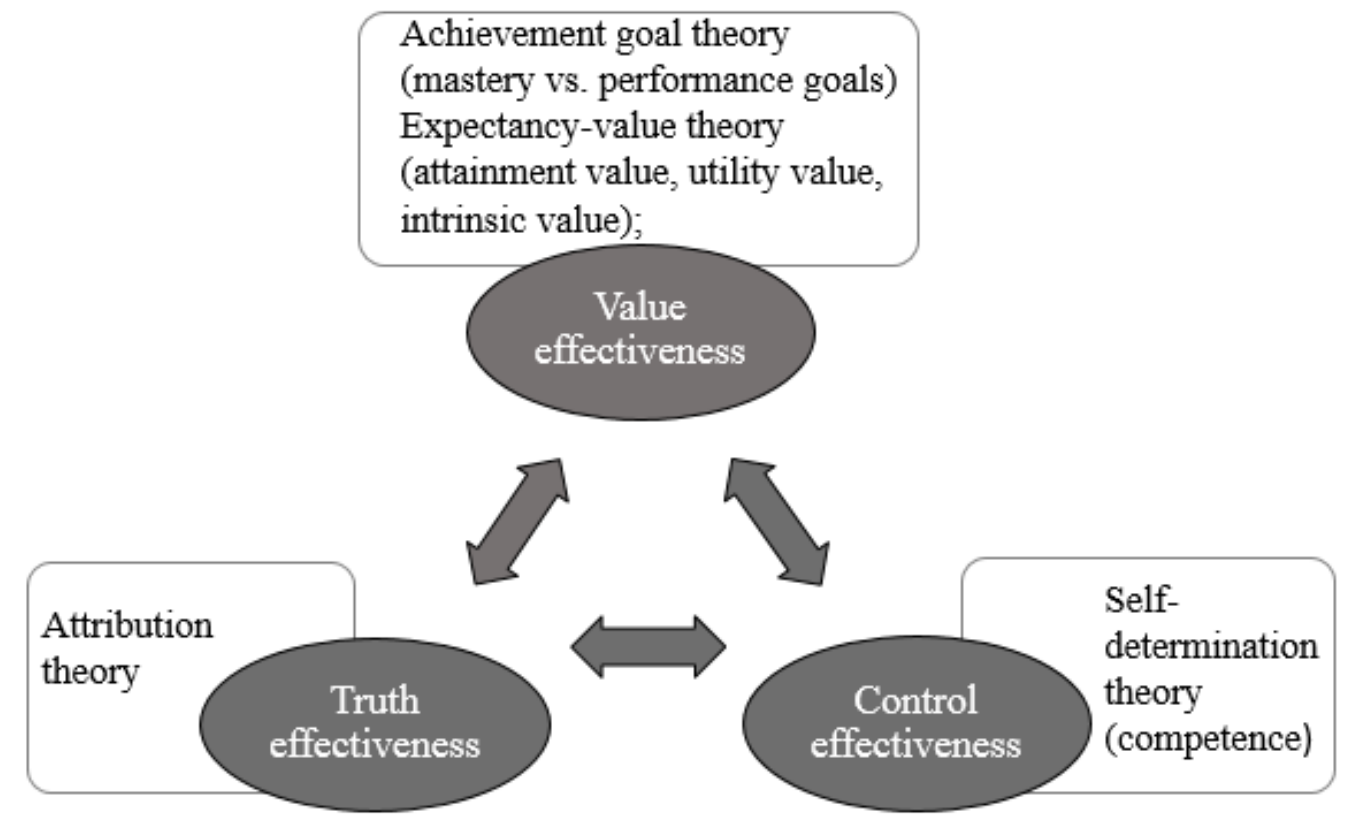

Figure 1. Placing Higgins' (2012) conceptualization of motivation in the field of motivation.

Distinctions among value, truth, and control effectiveness. Value, truth, and control effectiveness are distinct from one another. While value effectiveness is concerned with outcomes (e.g., benefits vs. costs), truth effectiveness is related to reality (e.g., real vs. imaginary, correct vs. false) and control effectiveness pertains to strength (e.g., strong vs. weak control over one's action; Higgins, 2012). What is important in terms of value effectiveness is ending with positive outcomes rather than how these outcomes are attained (Higgins, 2012, 2013). By contrast, truth and control effectiveness reflect the process of outcome attainment. For example, individuals constantly evaluate what they are doing during the goal pursuit and adopt different strategies to attain the goals. Additionally, value, truth, 
and control are associated with different motivational experiences (Higgins, 2012). Individuals feel cheerful and encouraged when achieving ideals and aspirations (i.e., when they attain promotion value) and feel upset and discouraged when failing to do so. In contrast, individuals feel quiescent and relaxed when they maintain safety and/or fulfill their responsibilities (i.e., when they achieve prevention value) and feel worried and anxious when they are unsuccessful in these undertakings (Higgins, 2012). Experiences related to truth and control success or failure are different from one another and from experiences of success/failure regarding value (Higgins, 2012). When individuals are effective in establishing the truth, they feel certain and confident; otherwise, they feel bewildered and suspicious. In contrast, when individuals succeed in having control effectiveness, they feel competent and powerful; otherwise, they feel helpless and incompetent (Higgins, 2012). Hence, individuals exhibit distinct feelings when experiencing success and failure with regards to having value, truth, and control effectiveness.

\section{Interrelationships among value, truth, and control effectiveness. Importantly,} strivings for value, truth, and control effectiveness do not exert isolated effects on motivation; on the contrary, they interact with one another to shape motivation effects (Higgins, 2012). The important motivation consequences of the interrelationships among value, truth, and control effectiveness are illustrated in the following example. A student wants to get an A in a course and regards this aim as an opportunity to advance (i.e., she strives for promotion value). She also believes that it is highly likely to attain an A because of her academic success history (i.e., she has high truth effectiveness) and works very hard by reading beyond the course requirement and seeking help from teachers and classmates (i.e., she exerts eager control). In this case, the eager control combined with the promotion value makes the student feel right and enhances the engagement in what she/he is doing (Higgins, 2012). This experience of feeling right includes an element of being true and correct (Higgins, 2012). Hence, this student may experience an increased truth effectiveness and feel more confident of the future success. Likelihood of success could function as reality preparation (Higgins, 2012). Preparing for future success (e.g., securing her dream job), this student will be motivated to be even more committed and to expend more effort to study. Therefore, she is likely to experience intensified control effectiveness. In turn, this strengthening of 
engagement and commitment may increase the value of getting an A. This example illustrates that motivation effects related to value, truth, and control effectiveness are shaped by the interrelationships among them (i.e., organization of motivation; Higgins, 2012).

As an organization of motivations, value, truth, and control effectiveness function not only independently but also concurrently because people are motivated to be effective with respect to all these motivational dimensions (Higgins, 2012). On the one hand, as noted above, these three types of effectiveness strivings play distinct motivation roles in shaping people's behaviors, cognition, and affective reactions. On the other hand, as each striving for effectiveness has its potential benefits and costs, they could either support or disrupt one another (Higgins, 2012; Higgins, Cornwell, \& Franks, 2014). In the example above, the means of pursuing an A (i.e. eager control) entail opportunities to maximize gains and, thus, sustains the goal of advancement (i.e. promotion value). In some other situations, effectiveness strivings could optimally constrain drawbacks associated with other types of effectiveness strivings. For instance, on one hand, strong truth motivation may provide appropriate direction for strivings for control effectiveness so as to make something desired happen (and something undesired not happen). On the other hand, strong control effectiveness may put a limit on potential downsides of truth motivation (e.g., getting stuck in excessive reflection and delaying in action) and motivate people to take action and manage what is needed to attain a given goal (Higgins, 2012; Higgins et al., 2014). Hence, these two ways of being effective could complement each other to motivate people to advance towards the most valuable outcome by means of the most effective strategies (Higgins, 2012).

Value, truth, and control can receive distinct emphases from different individuals in different situations and, thus, produce different motivation effects (Higgins, 2012). For instance, some students have a strong desire to get an A in an important course (i.e., have high value effectiveness). They also work hard to achieve their goal (i.e., have high control effectiveness). However, when the course is difficult, some students may perceive that they have a low likelihood of success (i.e., have low truth effectiveness). As long as success does not appear impossible, this low likelihood can function as an interference that obstructs goal attainment (Higgins, 2012). To succeed, students need to work harder to oppose the interfering force. When they do work harder, the low truth effectiveness along with high 
value and control effectiveness helps strengthen students' engagement in learning-related tasks (Higgins, 2012).

In sum, Higgins (2012) theorized that motivation is driven by the interrelationships among the three types of striving for effectiveness - the organization of motivations. Value, truth, and control work together to shape motivation effects. This perspective highlights that no motivation dimension can be considered in isolation; instead, the role of each motivation dimension depends on the strength of the other dimensions (Higgins, 2012). Therefore, to understand motivation and motivation effects, it is necessary to examine strivings for value, truth, control effectiveness, as well as their interrelationships (Higgins, 2012; Higgins et al., 2014).

Measuring strivings for value, truth, and control effectiveness. Regulatory focus theory (Higgins, 1997, 2012) distinguished between two motivation orientations (promotion and prevention), which are both associated with value effectiveness. Promotion orientation focuses on the value of advancement and accomplishment, the value of advancing from a neutral status quo ("0" or non-gain) to positive outcomes (“+1" or gain); prevention orientation concerns the value of safety and security, the value of maintaining a status quo (“0” or non-loss) and avoiding negative outcomes (“-1" or loss) (Higgins, 2012). In turn, regulatory mode theory (Kruglanski et al., 2000) differentiated two motivation modes (assessment and locomotion). The assessment orientation is related to strivings for truth effectiveness, because it gauges the extent to which individuals engage in making critical comparisons and evaluations to seek the truth. In contrast, the locomotion orientation is associated with strivings for control effectiveness, because it focuses on an individual's effectiveness with regarding to initiating and sustaining smooth and uninterrupted movement from the present circumstance/situation (Higgins, 2012). Higgins (2012) proposed that promotion, prevention, assessment, and locomotion are motivation tendencies that are at play in every individual and culture; together, these four orientations measure strivings for value, truth, and control effectiveness. Moreover, Higgins (2012) argued that promotion, prevention, assessment, and locomotion work together as an organization of motivations and, therefore, affect how people perceive and deal with the world. More details about these four orientations are included in the following sections. 
To sum up, this section highlighted Higgins' (2012) conceptualization of motivation as interacting strivings to be effective in life pursuits. As illustrated in Figure 2, this conceptualization includes the three types of effectiveness strivings (denoted with circles): value effectiveness (having desired outcomes), truth effectiveness (establishing the truth), and control effectiveness (having an impact, exerting an influence). These strivings are distinct from one another and work together to shape motivation effects. In this diagram, the interactions among value, truth, and control effectiveness are indicated by the two-way arrows, which suggest the interplay of the three components of motivation. Any motivation effect is driven by the interrelationships among value, truth, and control effectiveness (i.e., by the organization of motivation presented in the center of the diagram). Promotion, prevention, assessment, and locomotion, which are displayed in ovals, are key motivation orientations that measure these three ways of being effective.

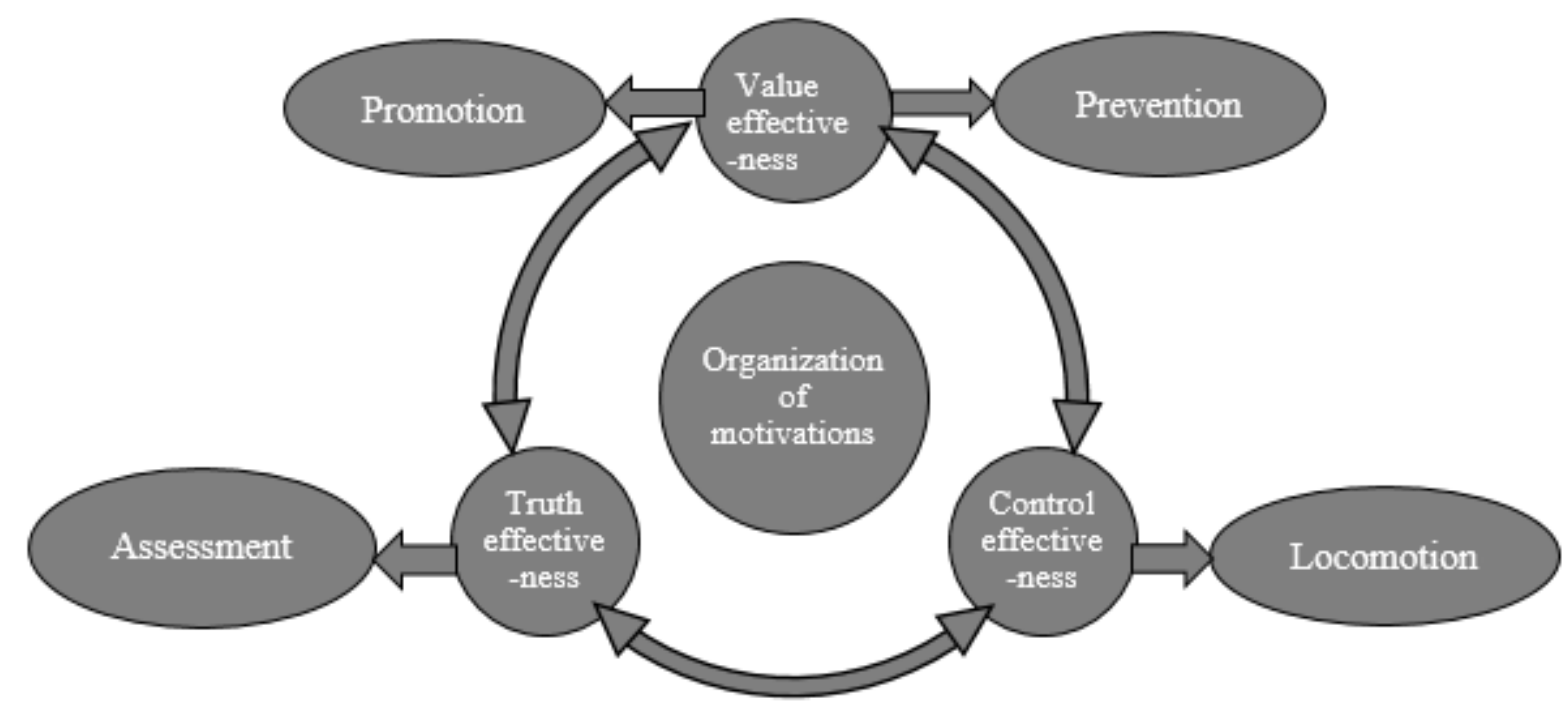

Figure 2. Schematic representation of Higgins' (2012) model of motivation.

\section{Regulatory Focus Theory}

This section presents an overview of Higgins' (1997) regulatory focus theory. In this theory, Higgins differentiated two motivation orientations (i.e., promotion focus and prevention focus). Promotion and prevention are shaped by early socialization experiences and cultural influences, but could also be activated temporarily by situational factors. People 
having a primarily promotion (vs. prevention) orientation prefer to use different strategies (i.e., eagerness vs. vigilance) to achieve their goals and experience different emotions when they are successful/unsuccessful in their goal pursuits (i.e., elation-dejection vs. quiescenceagitation).

Promotion and prevention orientations. Higgins (1997) postulated that promotion and prevention foci are distinct motivation tendencies that serve two fundamental survival needs, that is, nurturance (e.g., nourishing, growth, development) and security (i.e., protection, safety), respectively. The promotion focus is the motivation orientation related to nurturance needs. Individuals with a promotion orientation strive for growth, advancement, and accomplishment. In contrast, the prevention focus is the motivation orientation related to security needs. Individuals with a prevention orientation strive for safety and are motivated to fulfil duties, obligations, and responsibilities.

Regulatory focus theory is rooted in self-discrepancy theory (Higgins, 1987). Higgins (1987) identified three different self-representations that play important roles in selfevaluation and self-regulation: the actual self-states, the ideal self-states, and the ought selfstates. The actual self-states refer to the representations of attributes that an individual thinks she/he actually has. By contrast, the ideal self-states consist of attributes an individual believes she/he ideally has (i.e., representations of hopes, wishes, and aspirations); the ought self-states are attributes an individual thinks she/he ought to have (i.e., representations of duties, obligations, and responsibilities). According to Higgins (1987), the ideal and ought self-states are self-guides, that is, "self-directive standards or acquired guides for being" (Higgins, 1987, p. 321). Individuals evaluate their actual self-states against their ideal or ought self-guides and regulate their behaviors accordingly. In so doing, people develop two different self-regulatory systems: the ideal self-regulatory system (i.e., promotion focus) and the ought self-regulatory system (i.e., prevention focus). The former is related to an individual's ideal self-guide and is focused on the presence and absence of positive outcomes (i.e., the discrepancy vs. congruence between the actual self and the ideal self-guide). The latter is related to an individual's ought self-guide and is concerned with the presence and absence of negative outcomes (i.e., the discrepancy vs. congruence between their actual self and the ought self-guide). 
Accessibility of regulatory foci. Accessibility is "the ease with which an available mental representation (i.e., a structured set of information already encoded in memory) can be activated by external simulation" (Eitam, Miele, \& Higgins, 2013, p. 463). As noted above, promotion and prevention are apparent for every individual due to survival value of these two tendencies. The sources of promotion and prevention orientations could be either chronic accessibility (personality differences) or temporary accessibility (situational cues) (Scholer \& Higgins, 2011).

The chronic regulatory focus is a stable disposition that is, at least in part, shaped by socialization experiences with significant others and cultural contexts (Higgins, 2012; Molden \& Miele, 2008; Molden \& Rosenzweig, 2016). For example, when parental practices consistently involve reward and punishment in terms of the presence and absence of positive outcomes (e.g., parents give a hug for good behavior vs. parents show disappointment for bad behavior), children are likely to adopt an ideal self-guide and to become sensitive to gains; in turn, these aspects are likely to contribute to the development of a chronic promotion orientation. In contrast, when children's upbringing experiences often involve reward and punishment in terms of the presence and absence of negative outcomes (e.g., being criticized for bad behavior vs. avoiding punishment for good behavior), they are likely to adopt an ought self-guide and to become sensitive to losses; in turn, these aspects are likely to contribute to the development of a chronic prevention orientation (Higgins, 1997, 2012; Miele \& Wigfield, 2014). In cultural contexts where individuality and independence are highlighted (e.g., in the U.S), individuals may be more likely to be concerned with advancement and accomplishment and, thus, tend to be predominantly promotion focused. By contrast, in cultural contexts where interdependence and collective achievement are emphasized (e.g. in Japan), people may be more inclined to focus on security and responsibility and tend to be predominantly prevention focused (Higgins, 2012; Molden \& Miele, 2008; Molden \& Rosenzweig, 2016).

Individuals' regulatory focus orientations could also be induced temporarily by situational factors (Higgins, 1997; Molden, Lee, \& Higgins, 2008; Molden \& Rosenzweig, 2016). Characteristics of momentary situations (e.g., external incentives) may evoke temporarily either a promotion or prevention orientation. For instance, Higgins (1997) 
proposed that teachers' feedback or task instructions that highlight potential gains or positive outcomes tend to temporarily induce promotion orientations in students, whereas situational cues that highlight potential losses or negative outcomes tend to prime prevention orientations.

Differences between promotion and prevention orientations. As discussed previously, promotion and prevention orientations are both related to strivings for value effectiveness (i.e., having desired outcomes). However, the two motivation orientations differ at the system, strategic, and tactical levels of self-regulation and are associated with distinct emotional experiences for success and failure.

Motivational concerns. The system level refers to individuals' motivational concerns or goals (Scholer \& Higgins, 2011). At the system level, both promotion and prevention orientations involve approaching desired end-states (e.g., advancement vs. security) and avoiding undesired end-states (e.g., lack of fulfillment vs. threat); however, they differ in terms of the particular motivational concerns that are emphasized. Specifically, promotionfocused individuals pay particular attention to the presence and absence of positive outcomes (i.e., gains and non-gains). They represent goals as ideals, hopes, wishes, or aspirations and are motivated towards growth, advancement, and accomplishment. In contrast, preventionfocused individuals tend to be sensitive to the absence and presence of negative outcomes (i.e., non-losses and losses). They strive for ought goals (i.e., to fulfil duties, obligations, and responsibilities) and try to maintain safety. For instance, two students could have the same desired end-state (e.g., graduating from university). A promotion-focused student regards graduation as an accomplishment that advances personal growth, which is a gain to her. In contrast, a prevention-focused student considers this aim as a responsibility to secure a future career, which is a non-loss to her.

It should be noted that a neutral status quo (i.e., "0”) has a different valence for promotion and prevention orientations (Higgins, 2012, 2014). In the case of promotion orientation, attaining a positive outcome is a gain and thus has positive valence (i.e., "+1", a positive deviation from the status quo), while maintaining a status quo " 0 " has negative valence, because it involves no gains. By contrast, for prevention-focused individuals, maintaining a status quo "0" (i.e., the absence of negative outcomes, non-loss) has positive 
valence while failing to maintain a status quo " 0 " has negative valence (i.e., "-1" or loss).

Eagerness versus vigilance. Promotion and prevention orientations also differ at the strategic and tactical levels. Strategies are the general plans or means used to pursue goals, while tactics refer to the specific ways a strategy is implemented in a particular situation (Scholer \& Higgins, 2010). The same strategy can be implemented by different tactics in different contexts (Scholer \& Higgins, 2011). For instance, individuals could adopt either approach or avoidance tactics in the service of approach strategies.

At the strategic level, promotion and prevention orientations involve different preferences for goal pursuit strategies. Individuals with a history of promotion success tend to adopt eager approach strategies, whilst people with a history of prevention success tend to use vigilant avoidance strategies (Higgins et al., 2001). Eager approach strategies involve approaching matches to desired end-states and mismatches to undesired end-states. The eagerness is consistent with promotion-focused individuals' motivational concern with gains and their sensitivity to the difference between " 0 " and " +1 ". Therefore, promotion-focused individuals prefer eager approach strategies to achieve gains and strive for advancement and accomplishment (Higgins, 1997, 2012).

By contrast, vigilant avoidance strategies involve avoiding matches to undesired endstates and mismatches to desired end-states. The vigilance is related to prevention-focused individuals' motivational concern with losses and their sensitivity to the difference between " 0 " and " 1 ". Therefore, as long as they are in a neutral or satisfactory state, preventionfocused individuals prefer vigilant avoidance strategies to avoid mistakes and to protect against losses (Higgins, 1997, 2012). For example, a promotion-focused student regards getting an A in a course as an ideal goal and, therefore, may seek eagerly every possible opportunity to maximize gains (e.g., reading extra materials, receiving tutoring, and participating in study groups) even at the expense of making mistakes and facing losses. In contrast, a prevention-focused student regards getting an A in a course as an ought goal, and therefore, may avoid vigilantly any possible mistakes to minimize losses (e.g., following the course requirement, resisting distractions) even at the expense of missing opportunities for potential gains.

Different tactics can be adopted to support eagerness or vigilance in different 
situations. Generally, promotion-focused individuals are more inclined to use approach tactics and have a risky bias, while prevention-focused individuals tend to employ avoidance tactics and have a conservative bias (Crowe \& Higgins, 1997; Friedman \& Förster, 2001). For example, when faced with failure, promotion-focused individuals tend to increase their expectancy of future success to sustain eagerness, while prevention-focused individuals tend to lower their expectancy of future success to maintain vigilance (Higgins, 2012). However, when individuals in a prevention focus are in a negative condition (i.e., “-1”, negative deviation from the positive outcome) and conservative or avoidance tactics cannot ensure a return to the satisfactory state (i.e., "0"), it is more likely that they will adopt risky or approach tactics to restore the satisfactory non-loss (Higgins, 2012, 2014; Scholer \& Higgins, 2008). For example, in a study on stock investment, participants with a prevention orientation in a condition of loss were more willing to choose the risky stock to get back to the non-loss (i.e., break-even) situation (Scholer \& Higgins, 2008).

Emotional sensitivities. According to regulatory focus theory, promotion and prevention orientations could sensitize individuals to different emotions. Moreover, the emotions being experienced vary in intensity (Higgins, 1997; Molden et al., 2008; Molden \& Miele, 2008). When individuals attain promotion success (e.g., achieve ideal goals), they experience elation-related feelings of high intensity such as happiness and cheerfulness. When individuals achieve prevention success (e.g., fulfil duties and responsibilities), they have quiescence-related emotions of low intensity such as calmness or relaxation. In contrast, when individuals fail in pursuing promotion concerns, they experience less intense dejectionrelated feelings such as being disappointed or sad. When individuals fail to attain prevention goals, they experience more intense agitation-related emotions such as fear or anxiety (Higgins, 1997, 2012).

In sum, promotion and prevention orientations have different characteristics. As shown in Figure 3, these two orientations both involve approaching desired end-states (illustrated in the right part of the diagram) and avoiding undesired end-states (illustrated in the left part of the diagram), but they are associated with different concerns, preferences for using distinct types of strategies and tactics for goal pursuit as well as experiencing distinct emotions for success and failure. The top half of Figure 3 illustrates the characteristics of a promotion 
orientation. Rooted in the nurturance need, a promotion orientation is sensitive to gains vs. non-gains. Promotion-focused individuals strive for ideal goals and prefer to use eagerness to maximize gains and avoid non-gains. Moreover, promotion success (i.e., gains) elicits elation, while promotion failure (i.e., non-gains) elicits dejection. The bottom half of this diagram shows the characteristics of a prevention orientation. Rooted in the security need, a prevention orientation is sensitive to losses and non-losses. Prevention-focused individuals strive for ought goals and prefer to use vigilance to minimize losses. Moreover, prevention success (i.e., non-losses) elicits quiescence, while prevention failure (i.e., losses) elicits agitation.

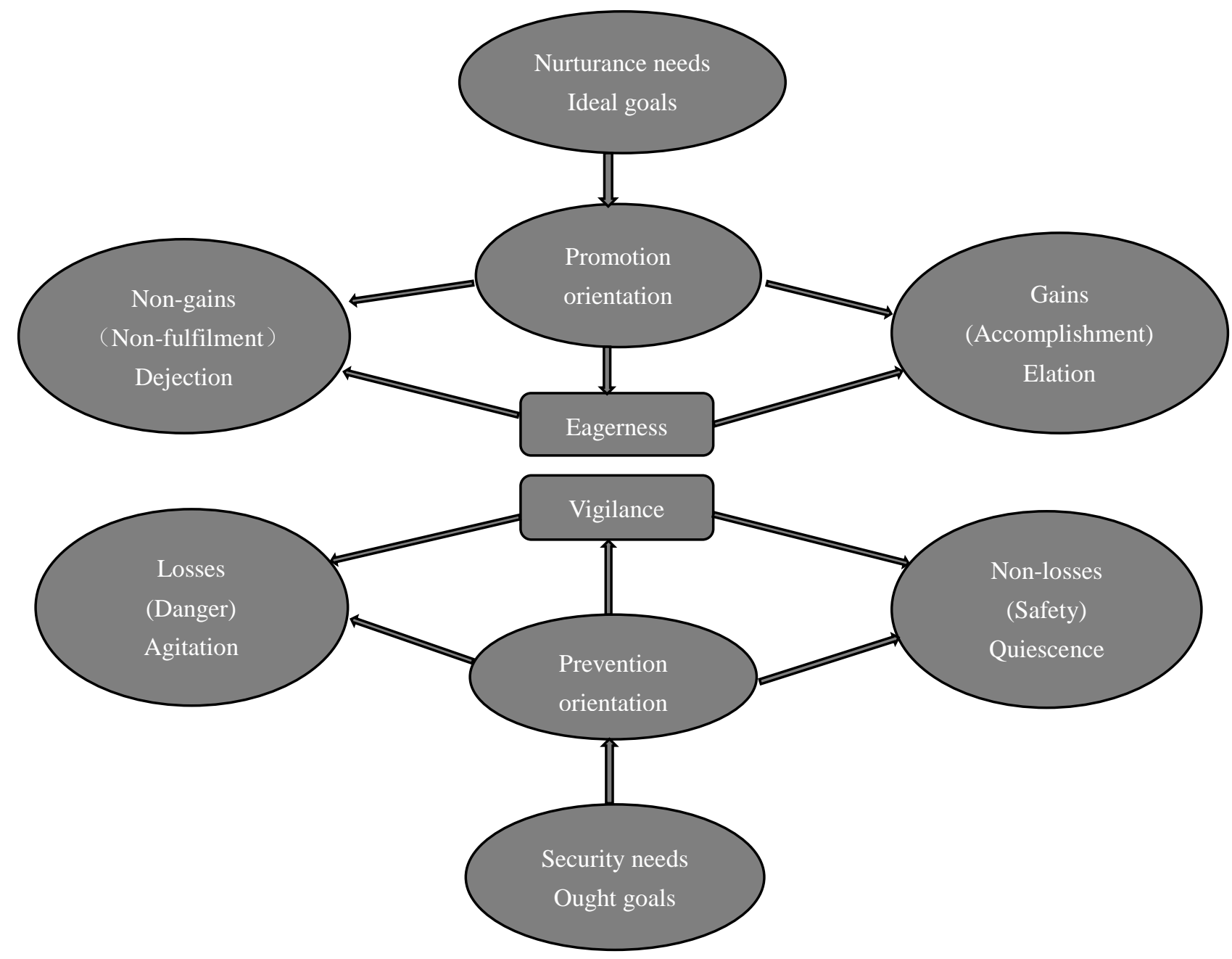

Figure 3. Schematic representation of the conceptualization of promotion and prevention orientations.

Neither orientation is more adaptive than the other; both have strengths and costs 
(Molden et al., 2008; Molden \& Rosenzweig, 2016; Scholer \& Higgins, 2010). For example, because promotion-focused individuals strive to maximize gains, they tend to consider a larger number of alternative hypotheses in decision making and propose more alternative solutions in problem solving. In contrast, motivated to minimize losses, prevention-focused individuals prefer to narrow down the alternative hypotheses in making decisions. Therefore, individuals with promotion concerns are more open-minded and creative than preventionfocused individuals (Crowe \& Higgins, 1997). However, promotion-focused individuals' openness to different alternatives could also result in uncertainty and indecision, while prevention- focused people's cautious analysis could lead them to certainty and accuracy (Molden et al., 2008). Therefore, in the light of the trade-offs of promotion and prevention orientations, it is important to consider which orientation and strategy best fit the task demands (Molden et al., 2008) and whether the two orientations could complement each other to facilitate success (Molden \& Miele, 2008; Molden \& Rosenzweig, 2016). The relationships between promotion and prevention orientations are discussed next.

Relationships between promotion and prevention orientations. Higgins (2012, 2014) suggested that promotion and prevention orientations exist in every individual, but vary in strength. They are independent variables rather than two separate ends of a continuum (Scholer \& Higgins, 2008). Therefore, for an individual, both of the orientations could be strong, or both could be weak, or one could be strong and the other weak (Higgins, 2012; Shu \& Lam, 2011). Higgins (2014) suggested that when both promotion and prevention orientations are activated in an individual, the two orientations can function together effectively, each compensating for the downside effects of the other. For example, individuals who have strong focus on both orientations are fast and accurate in information processing (Förster, Higgins, \& Bianco, 2003).

Regulatory fit. Higgins (2012) posited that individuals experience regulatory fit when the strategies they use to pursue their goals match their regulatory focus orientations. For instance, there is a fit between eager (vigilant) strategies and the promotion (prevention) orientation. As discussed above, the promotion orientation focuses on the presence or absence of positive outcomes. As eager strategies ensure gains and guard against non-gains, eager strategies sustain a promotion focus (Higgins, 2012). Similarly, vigilant strategies sustain a 
prevention orientation. When people experience regulatory fit, they feel right, are more engaged in what they are doing and experience the task in which they are engaged as more valuable or important (Higgins, 2012).

As previously discussed, promotion and prevention orientations are both concerned with value. Eager and vigilant strategies are associated with strivings for control. Therefore, regulatory fit between the promotion (prevention) value and eager (vigilant) control provides a good example of motivations working together during goal pursuit (Higgins, 2012).

Importance of promotion and prevention in motivation regulation. Motivation regulation is the process by which individuals monitor and control the level (i.e., low vs. high) and the type (e.g., promotion vs. prevention, intrinsic vs. extrinsic) of their motivation to attain important goals (Miele \& Scholer, 2018; Scholer, Miele, Murayama, \& Fujita, 2018). Promotion and prevention orientations are "fundamental for understanding how people regulate motivation” (Miele \& Scholer, 2018, p. 7).

As noted before, the regulatory focus orientations fulfil basic survival needs (i.e., growth vs. security) and shape the types of goals people set (i.e., ideal vs. ought goals) and the strategies they adopt during goal pursuits (i.e., eagerness vs. vigilance). Hence, each of these orientations may be adaptive to different kinds of tasks (Higgins, 2012). For example, when a task demands creativity (e.g., brainstorming ideas for an essay), a promotion focus may lead to optimal performance as it motivates individuals to be more creative and generate a broad set of new ideas (Molden \& Rosenzweig, 2016). In contrast, when a task requires accuracy (i.e., writing an argument), a prevention focus may be more adaptive as it motivates people to be more careful against mistakes and possibly craft logically coherent arguments (Molden \& Rosenzweig, 2016).

One important aspect of effective motivation regulation entails identifying and deploying in real time the type of motivation orientation that matches the particular demands of a task at hand (Miele \& Scholer, 2018; Scholer et al., 2018). For example, during task engagement, if a student realizes that her promotion focus does not suit well the demands of writing a logical argumentation, she needs to regulate her motivation by shifting into a prevention orientation (e.g., inducing a prevention focus by thinking about the consequences that ensue if she fails). In doing so, there will be a regulatory fit between the type of 
motivation she is experiencing and the demands of the current task. In turn, this fit will help strengthen her task engagement and enhance her performance (Higgins, 2012; Miele \& Scholer, 2018).

Another effective way of regulating motivation is to monitor metamotivational feelings (i.e., particular feelings associated with distinct types of motivations) in order to understand the type and strength of motivation experienced (Miele \& Scholer, 2018). For example, feelings of hope and cheerfulness may signal having a strong promotion focus, whilst feelings of tension and anxiety may indicate having a high level of prevention orientation (Higgins, 1997, 2012).

Taken together, promotion and prevention orientations are important motivation components that individuals can monitor and adjust during task engagement (Miele \& Scholer, 2018). Hence, knowledge of these two regulatory foci is critical for understanding key aspects that optimally support motivation regulation.

\section{Distinguishing regulatory focus from approach and avoidance motivations.}

Promotion and prevention orientations are not equivalent to approach and avoidance motivations (Miele \& Wigfield, 2014; Molden \& Miele, 2008). An approach motivation is focused on attaining positive end-states and an avoidance motivation is concerned with avoiding negative end-states (Elliot \& Covington, 2001). In contrast, at the system level, both promotion and prevention orientations involve approaching desired (i.e., positive) and avoiding undesired (i.e., negative) end-states. Both approach and avoidance goals can be pursued by either a promotion focus or a prevention focus. An example is the case of students who have the same desired end-state (e.g., to graduate from university). Promotion-focused students perceive this goal as an accomplishment that they aspire to attain, while preventionfocused students treat it as a responsibility that they ought to fulfil. Similarly, students who try to avoid an undesired end-state (e.g., fail to graduate from college) could, with a promotion focus, regard this failure as an unseized opportunity for advancement; in contrast, with a prevention focus, students could perceive this goal as avoiding an important threat to their future careers.

At the strategic and tactical levels, both promotion-focused and prevention-focused individuals can adopt approach or avoidance strategies and tactics to pursue approach 
(avoidance) goals. Promotion-focused individuals prefer using eager approach strategies, but nothing stops them from using vigilant strategies or tactics (Scholer \& Higgins, 2008). Prevention-focused individuals are motivated to employ vigilant avoidance strategies; however, in negative circumstances they will use approach tactics to return to safety. By contrast, avoidance motivation is associated with only avoidance behaviors (Elliot \& Covington, 2001). Moreover, whilst approach and avoidance motivations are related to positive and negative affect respectively, promotion and prevention orientations are associated with both positive and negative emotions (Elliot \& Covington, 2001; Higgins, 2012).

To conclude, regulatory focus theory extends approach and avoidance motivation models by specifying different types of positive and negative outcomes (e.g., pertaining to aspirations and duties, respectively) and different ways to approach positive outcomes and avoid negative ones. In so doing, regulatory focus theory distinguishes between prevention and promotion orientations. Each orientation is characterized by different motivational concerns (gains vs. losses), emotional experiences at success/failure (elation-dejection vs. quiescence-agitation), and preferred strategies (eager approach vs. vigilant avoidance) for goal pursuit (Higgins, 2012). Together, promotion and prevention orientations index value effectiveness.

\section{Regulatory Mode Theory}

This section presents an overview of regulatory mode theory (Kruglanski et al., 2000). Regulatory mode theory differentiated two motivation orientations (i.e., assessment and locomotion) as distinct and independent self-regulatory modes that underlie most goal pursuit activities. According to traditional control models, individuals need to assess alternative goals and means in order to locomote (i.e., move) towards their desired goals (Carver \& Scheier, 1981). This is why these theories conceptualize assessment and locomotion as inseparable and interdependent facets of self-regulation (Kruglanski et al., 2000). In contrast, regulatory mode theory posited that assessment and locomotion orientations are independent of each other; moreover, individuals can perceive assessment and locomotion as ends in themselves 
(Higgins et al., 2003; Kruglanski et al., 2000). Assessment and locomotion orientations represent chronic individual differences; nevertheless, each orientation could be induced momentarily by situational factors (Kruglanski, Orehek, Higgins, Pierro, \& Shalev, 2010). These two motivation orientations are associated with different motivational characteristics and exert independent effects on goal setting and goal pursuit (Higgins et al., 2003; Kruglanski et al., 2000; Pierro et al., 2018).

Assessment and locomotion orientations. Assessment is defined as "the comparative aspect of self-regulation concerned with critically evaluating entities or states, such as goals or means, in relation to alternatives in order to judge relative quality" (Kruglanski et al., 2000, p. 794). Having an assessment orientation is associated with strivings for truth effectiveness (Higgins, 2012). When assessment is strong, making comparisons is valued as an end in itself. The essential motivation for assessors is to 'do the right things' (Kruglanski et al., 2000); thus, they are concerned with making critical comparisons and evaluations of alternative goals and means as well as of their competencies and performance to ensure that they make the correct choice (Higgins et al., 2003).

Locomotion is defined as "the self-regulatory aspect concerned with movement from state to state and with committing the psychological resources that will initiate and maintain goal directed progress in a straightforward manner, without undue distractions or delays" (Kruglanski et al., 2000, p. 794). Having a locomotion orientation is related to strivings for control effectiveness (Higgins, 2012). The essential motivation for locomotors is to 'just do it' (Kruglanski et al., 2000). As locomotion involves initiating and maintaining smooth and uninterrupted movement, this orientation illustrates people's strivings to exert control over their actions and the environment. Locomotors can be motivated to move towards a desired end-state in some cases, but they can value moving from current states as an end in itself and strive to avoid immobility (Higgins et al., 2003).

Precursors of assessment and locomotion orientations. Regulatory mode theory proposed that assessment and locomotion orientations are motivational states that vary across individuals and situations (Higgins, 2012). Individuals have chronic differences in the strength and predominance of assessment and locomotion tendencies; these differences are rooted in their temperaments and socialization experiences (Kruglanski et al., 2010). For 
instance, children's interactions with their significant others create a shared reality concerning how much assessment or locomotion should be emphasized in self-regulation. If this shared reality often involves emphasizing the importance of doing 'the right things', children tend to be predominantly assessment oriented; in contrast, if this shared reality is centered on 'doing things rather than doing nothing', children are inclined to be predominantly locomotion oriented (Higgins, 2008). Moreover, situational factors could induce either assessment or locomotion to become the preferred motivational orientation in goal pursuit (Higgins et al., 2003). For example, activities such as problem solving and idea generation, which involve critical comparisons and evaluations, could induce an assessment orientation to these tasks; activities such as playing sports, which involves movement, could induce a locomotion orientation (Higgins, 2008).

\section{Motivational characteristics of assessment and locomotion orientations. As}

discussed previously, individuals having a strong assessment orientation are fundamentally concerned with making critical comparisons, while individuals with a strong locomotion orientation pay particular attention to movement from state to state. Therefore, these two orientations are related to distinct motivational characteristics.

Motivational characteristics of assessment. Because assessment involves making comparisons, having a strong assessment orientation is characterized by elevated selfevaluative concerns (Higgins et al., 2003). In addition, individuals having a strong assessment orientation focus on comparing and evaluating their actual self-states against multiple sets of standards including social norms and standards. Moreover, they tend to compare their performance with others' and thus have a performance orientation. Due to their concerns with the evaluative consequences of their performance, assessors are generally driven by extrinsic motivations (Higgins et al., 2003; Kruglanski et al., 2000). The self-evaluation concerns of individuals who have a strong assessment orientation are associated with low levels of selfesteem and optimism and make these individuals vulnerable to negative affect (e.g., anxiety and depression) and emotional instability (Higgins et al., 2003; Kruglanski et al., 2000). Furthermore, strong assessment is associated with reduced decisiveness in decision making (Kruglanski et al., 2000) and with preference for exhaustive evaluation strategies (i.e., comparing and evaluating all available options on various dimensions of interest at the same 
time; Higgins, 2012; Molden, 2012). This is the case because high levels of assessment prompt tendencies to expend longer time and more effort to compare, evaluate, and choose optimal goals and effective means (Higgins et al., 2003; Kruglanski et al., 2000).

Motivational characteristics of locomotion. Locomotion orientation focuses psychological resources on initiating and maintaining smooth movement (i.e., flow) and, thus, is characterized by activity flow concerns (Higgins et al., 2003). Having a strong locomotion orientation is associated with a mastery orientation because mastering or learning new knowledge or skills involves forward movement and change (Higgins et al., 2003). Locomotors also tend to be intrinsically motivated because their activity flow concerns enhance their experiential involvement in tasks and make them feel autonomous (Higgins et al., 2003; Kruglanski et al., 2000). To initiate movement, locomotors are decisive and open to change so as to make action proceed; to sustain steady movement, locomotion is associated with persistence and vitality (Higgins et al., 2003). As a consequence, individuals having strong locomotion orientation generally experience positive affect, high self-esteem, and optimism (Higgins et al., 2003; Kruglanski et al., 2000). Due to concerns with progress, locomotors tend to prefer strategies of progressive elimination and decisive action (Higgins, 2012; Molden, 2012). For instance, high locomotors are adroit at narrowing down the number of alternatives and making a quick selection of means to start a task or an activity (Kruglanski et al., 2000).

Table 2

Motivational Characteristics of Assessment and Locomotion Orientations

\begin{tabular}{lllllll}
\hline $\begin{array}{l}\text { Regulatory } \\
\text { mode }\end{array}$ & \multicolumn{5}{c}{ Motivational characteristics } \\
\hline Assessment & $\begin{array}{l}\text { Self- } \\
\text { evaluation } \\
\text { concerns }\end{array}$ & $\begin{array}{l}\text { Performance } \\
\text { orientation }\end{array}$ & $\begin{array}{l}\text { Extrinsic } \\
\text { motivation }\end{array}$ & $\begin{array}{l}\text { Less } \\
\text { decisive- } \\
\text { ness }\end{array}$ & $\begin{array}{l}\text { Negative affect, } \\
\text { low self-esteem, } \\
\text { and optimism }\end{array}$ & $\begin{array}{l}\text { Strategies of } \\
\text { exhaustive } \\
\text { comparisons }\end{array}$ \\
& Activity & Mastery & Intrinsic & High & Positive affect, & $\begin{array}{l}\text { Strategies of } \\
\text { Locomotion } \\
\end{array}$ \\
& flow & orientation & motivation & decisive- & high self- & progressive \\
& concerns & & & ness & esteem, and & eliminations \\
& & & & & optimism & \\
\hline
\end{tabular}

The main motivational characteristics of assessment and locomotion are summarized in Table 2. Similar to promotion and prevention orientations, assessment and locomotion 
orientations also have trade-offs. Having high levels of assessment may prompt both adaptive and maladaptive tendencies. For example, on one hand, engagement in critical selfevaluations may enable high assessors to obtain an accurate knowledge of their strengths and weaknesses, which could help them identify areas that need improving (Higgins et al., 2003). On the other hand, high assessors' preoccupations with excessive comparisons and evaluations could make them overthink, feel remorse or uncertainty, and delay taking action (Higgins, 2012; Pierro et al., 2018).

Similarly, locomotion is associated with both potential benefits and downsides. Specifically, strong locomotion may motivate people to engage in goal-related action quickly instead of procrastinating (Higgins, 2012; Pierro, Giacomantonio, Pica, Kruglanski, \& Higgins, 2011). However, locomotion focuses on movement itself and not on attaining any particular outcomes (Higgins, 2012). When the motivation to move from the current state becomes overly strong (and is not constrained by tendencies associated with other motivation orientations), individuals are likely to spring into action too fast without carefully considering whether they are moving toward the right direction and may end up in a worse condition (Higgins, 2012). Therefore, it is important to consider if the two orientations could complement each other to facilitate optimal performance (Higgins, 2012; Pierro et al., 2018). The relationships between assessment and locomotion orientations are discussed next.

Relationships between assessment and locomotion orientations. As noted earlier, assessment and locomotion orientations are motivational states that vary across individuals and situations. Therefore, individuals place different emphases on these two orientations (Higgins et al., 2003). Although assessment and locomotion play different roles in selfregulation and could even have opposite effects on goal pursuit, these two orientations can work together by complementing each other to achieve optimal performance (Higgins, 2012; Kruglanski et al., 2010). Specifically, careful assessment of the best goal to pursue or the most appropriate means to achieve the goal could contribute to goal attainment by ensuring that moving (i.e., locomotion) is not without direction. Likewise, locomotion could lead people to action and stop them being lost in thought (i.e., put a limit on assessing too much). The complementary role of these two orientations in goal pursuits was illustrated by findings indicating that students having high levels of both of these orientations were more likely to 
achieve better academic outcomes than those who had high levels of only one or neither orientation (Kruglanski et al., 2000). Successful goal pursuit that involves both assessment (i.e., truth effectiveness) and locomotion (i.e., control effectiveness) provides an additional example of these two tendencies working together effectively (Higgins, 2012).

\section{Interrelationships between Promotion, Prevention, Assessment, and Locomotion Orientations}

Being effective in life pursuits does not mean striving to maximize value, truth, and control effectiveness respectively (Higgins et al., 2014). As I will discuss shortly, effectiveness in life pursuits involves value, truth, and control working together to facilitate optimal self-regulation and effective goal pursuit (Higgins, 2012). Higgins (2012) proposed that promotion, prevention, assessment, and locomotion are key motivation orientations that measure value, truth, and control effectiveness, respectively. Although each of these orientations has distinct effects on motivation and the effectiveness of goal pursuit, promotion, prevention, assessment, and locomotion work together to shape motivation effects (Higgins, 2012). For example, during information processing, motivated by their concern with maximizing gains, individuals who have a strong promotion orientation attempt to consider all relevant information that appears correct and, thus, risk including incorrect information among the aspects they evaluate. In line with their dominant regulatory mode orientation, when examining this information, these individuals could adopt either an assessment-focused strategy (based on thorough comparison and analysis of each piece of information) or a locomotion-focused strategy (comprising fast progressive elimination of successive pieces of information that appear blatantly incorrect). Therefore, individuals having high levels of promotion and stronger assessment than locomotion are likely to spend a large amount of time critically comparing and evaluating at once the entire corpus of all the available information in order not to miss any possible correct information. In contrast, individuals having high levels of promotion and stronger locomotion than assessment are inclined to pursue the same goal (i.e., not to miss correct information) by processing all the information sequentially and at a high pace. This example illustrates how promotion, 
assessment, and locomotion simultaneously and interdependently work together to shape individuals' motivations to process information (Molden, 2012).

Higgins and his colleagues have argued that promotion, prevention, assessment, and locomotion interact (i.e., work together) to shape motivation effects (Cornwell, Franks, \& Higgins, 2015; Higgins, 2012). One way in which these four motivation orientations interact is by supporting and sustaining one another. For example, regulatory fit involving a strong locomotion orientation could strengthen the promotion or prevention value individuals attribute to a task (Higgins, 2012). Specifically, if locomotors are engaged in a task in a way that ensures fast and steady progress, this strategy sustains (i.e., fits) their locomotion concerns. According to the principles of regulatory fit theory (Higgins, 2012), regulatory fit is associated with increased engagement and enhanced valuation of the task/ its associated outcomes. Importantly, this increased value could be characterized as either promotion value or prevention value, depending on which of the two regulatory focus orientations is stronger.

Because each of these four motivation orientations has both potential benefits and costs, another way in which they could interact is by constraining or compensating for the downsides of the other orientations. When this process of compensating or constraining is effective, it contributes to optimal self-regulation (Cornwell et al., 2015; Higgins, 2012). For instance, when people perceive that there is a low likelihood of success in a task, promotion concerns with advancement might motivate them to abandon the original important goal (related to the attainment of the given task) and strive for new goals (Molden et al., 2008). In this situation, a strong prevention orientation, which is concerned with the fulfilment of responsibilities, could keep in check individuals' tendency to abandon important goals and motivate them to be more committed to the original goal pursuit (Molden et al., 2008).

Importantly, to facilitate optimal self-regulation by means of supporting the strengths of the other orientations and constraining their drawbacks, each orientation needs to have a certain strength (Cornwell et al., 2015). When one orientation is too weak or too strong, (either in absolute terms or relative to the magnitudes of the other three motivation orientations), it can neither fulfil its role in self-regulation nor compensate for the strengths of the other orientations. In these situations, individuals could experience motivational dysfunction (Cornwell et al., 2015). For instance, if locomotion is too weak, it fails to initiate 
or maintain psychological movement needed to drive or support change after failure. In addition, low locomotion cannot constrain the downsides of excessively strong assessment (e.g., getting lost in thought, delaying taking any action, and ruminating). As another example, individuals with disproportionately strong assessment concerns could be overly self-critical. When a dominant assessment is associated with a strong promotion orientation, this could lead to individuals having high actual-ideal discrepancies. In cases of systematic or catastrophic failures in their promotion goals, these individuals could experience depression, lose confidence, expect generalized failure, and become disengaged from activities that could lead to desired outcomes. Similarly, pairing a dominant assessment with a strong prevention orientation could lead to individuals having high actual-ought discrepancies. In circumstances of systematic or devastating failures in their prevention goals, these individuals could experience anxiety and become overly vigilant. On the other hand, when locomotion is overly strong and dwarfs assessment, individuals could move forward without going in the right direction (Higgins, 2012). As a result, they may move to any state that facilitate change, even if that change is not in the service of promotion or prevention goals (Cornwell et al., 2015).

In sum, promotion, prevention, assessment, and locomotion are fundamental survival factors that are represented in each individual's motivation (Higgins, 2008, 2012). These motivation orientations play different roles in self-regulation and goal pursuit and work together to shape motivation effects (Higgins, 2012). Therefore, to understand motivation and motivation effects, it is necessary to investigate promotion, prevention, assessment, and locomotion as well as their interrelationships (Cornwell et al., 2015).

\section{Motivation profiles defined by promotion, prevention, assessment, and}

locomotion. One effective way to understand the complex interrelationships among these four motivation orientations and their attendant motivation consequences is to analyze the motivation profiles that can be identified in a given population. A motivation profile gauges the magnitude of each orientation, and, thus, summarizes both the absolute and relative strengths of promotion, prevention, assessment, and locomotion. For example, an individual's motivation profile could comprise very strong promotion, assessment, and locomotion, and as well as very weak prevention (Hodis, 2018a). Investigating motivation profiles could help enhance the understanding of how these motivation orientations interact (i.e., work together) 
to shape self-regulation (Harring \& Hodis, 2016; Hodis, 2018a; Hodis et al., 2017; Pierro et al., 2018). In the case of the above-mentioned profile, the prevention orientation would be too weak to contribute to the self-regulation, let alone to compensate for the strengths of the remaining tendencies. Therefore, motivation effects associated with this motivational profile are most likely to be shaped by is the combination of very strong promotion, assessment, and locomotion (Hodis et al., 2017). Specifically, high levels of promotion may motivate this individual to eagerly strive for ideal goals. Her strong locomotion may counterbalance some of the self-regulatory downsides associated with having high assessment tendencies (e.g., excessively comparing and evaluating) and motivate her to initiate timely action to pursue a goal. However, she is likely to abandon an original goal when she faces difficulties or obstacles during a goal pursuit. This is the case because her strong promotion may make her switch to other goals if other goals appear more conducive for advancement. This lack of commitment to an original goal can be exacerbated by her strong locomotion. When she experiences no progress, her high level of locomotion is likely to motivate her to pursue other goals that could facilitate smooth movement.

In light of these considerations, motivation profiles are important for investigating the interrelationships among promotion, prevention, assessment, and locomotion. In addition, examining the motivation profiles identified in a target population could provide new information on how these orientations interact to shape inter-individual differences with regard to other motivation factors (e.g., grit, theory of intelligence, critical thinking, effort regulation, and perceptions/evaluations of academic success). Specifically, it can be analyzed whether distinct profiles are associated with different average levels on some (or all) of these constructs. These aspects will be examined in this thesis.

A hypothetical example will illustrate some important benefits that derive from conducting these analyses. For instance, student A might have a motivation profile that comprises high/very levels of promotion, assessment, and locomotion and very low levels of prevention (Hodis, 2018a). Results may show that she has a relatively strong incremental view of intelligence, does not display very high levels of grit, and engages strongly in critical thinking in her studies (see definitions of these motivation factors in Table 1). In contrast, student B might have a motivation profile that consists of low/very low levels of promotion, 
assessment, locomotion, and below average levels of prevention (Hodis, 2018a). Findings may show that she holds a stronger entity view of intelligence (compared to her incremental view of intelligence) and shows low levels of grit and critical thinking. Exploring differences between the motivation profiles of these two individuals sheds light on how promotion, prevention, assessment, and locomotion interact to shape motivation effects. For instance, it is likely that student A, motivated by her strong promotion concerns for growth and strong locomotion concerns for progress, believes her intelligence can be improved through effort. However, when facing obstacles, she is unlikely to be very gritty in pursuing her goals because strong promotion and locomotion tendencies may motivate her to give up the original goals (especially when facing difficulties) and select other goals that could offer better opportunities for advancement and uninterrupted movement. In addition, having high levels of assessment, she may spend a lot of time evaluating the knowledge she has learned in her courses and, thus, have high levels of critical thinking. By contrast, in the case of student $\mathrm{B}$, as all four motivation tendencies are weak or below average, she is likely to be at risk for amotivation and disengagement from studies (Hodis, 2018a; Hodis et al., 2017). Hence, she is not likely to be gritty and engage in critical thinking in her studies. Moreover, because her prevention is slightly higher than the other three tendencies, it is likely that she believes that her intelligence is by and large fixed.

To conclude, identifying and examining individuals' motivation profiles defined promotion, prevention, assessment, and locomotion contribute to understanding how these motivation orientations interact and how these interactions shape self-regulation, other motivation factors, and motivation effects. In turn, this new knowledge helps advance existing knowledge of motivation (Hodis, 2018a; Hodis et al., 2017; Pierro et al., 2018).

\section{Relationships between Motivation Orientations and Key Outcomes}

In this section, I review the literature on the key motivation factors (outcomes) explored in this study (i.e., grit, theories of intelligence, critical thinking, effort regulation, and perceptions/evaluations of academic success). Informed by the regulatory focus theory (Higgins, 1997, 2012) and the regulatory mode theory (Kruglanski et al., 2000), I propose 
some hypotheses regarding the relationships between promotion, prevention, locomotion, and assessment (on the one hand) and these outcomes (on the other). In addition, I use these hypotheses to make predictions regarding the extent to which distinct patterns of associations among the four orientations (i.e., different motivation profiles) identified in recent research are likely to be more (vs. less) supportive of grit and effort regulation. However, I am unable to make predictions with respect to theories of intelligence, critical thinking, and perceptions/evaluations of academic success, as few substantive studies provided empirical support for hypothesizing precise relationships between patterns of associations among the four orientations (on the one hand) and these constructs (on the other).

Grit. Grit is a relatively new construct in the psychological literature (Duckworth et al., 2007; Duckworth \& Quinn, 2009). Duckworth et al. (2007) proposed that grit reflects an individual's "perseverance and passion for long-term goals" (p. 1087). Some researchers have studied grit as a unitary construct, whilst some others have examined it as a construct composed of two facets, namely, perseverance of effort and consistency of interest (Duckworth et al., 2007; Duckworth \& Quinn, 2009). The former refers to the tendency to work hard in spite of obstacles, challenges, and failures; the latter describes the tendency to focus on the same long-term goal or interest.

Recent studies on the factor structure of grit have provided some empirical support for the second conceptualization of grit (Muenks, Wigfield, Yang, \& O’Neal, 2017; Steinmayr, Weidinger, \& Wigfield, 2018). For example, Muenks and her colleagues (2017) examined three measurement models of grit, namely, a one factor model in which grit is a unitary construct, a two correlated-factor model in which the perseverance of effort and consistency of interest are separate but correlated constructs, and a bi-factor model in which grit is a single construct that consists of two facets. These researchers reported that the bi-factor model fit best for the sample of university students (Muenks et al., 2017). These findings suggest that perseverance of effort and consistency of interest are two facets (i.e., subcomponents) of grit, as conceptualized by Duckworth and her colleagues (2007).

Grit has been found to be different from some other constructs related to success in school and in life. For instance, results reported by Duckworth and Quinn (2009) revealed that grit was positively associated with conscientiousness, but differed in its emphasis on 
perseverant pursuit of long-term goals (Duckworth et al., 2007). Grit is also different from resiliency in that the latter focuses on individuals' responses to adversity and stress, but does not require consistency of interest (Robertson-Kraft \& Duckworth, 2014). Moreover, Duckworth and Gross (2014) differentiated between grit and self-control. Specifically, they proposed that self-control is needed to resolve the conflict between lower-level and shortterm goals in daily life; by contrast, grit is required to pursue higher-level and long-term goals over time in spite of setbacks and failure (Duckworth \& Gross, 2014).

In contrast, recent empirical studies suggest that grit is very similar conceptually and operationally to some important constructs studied in personality, self-regulation, and engagement research (Credé, 2018). For instance, Credé, Tynan, and Harms (2017) conducted a meta-analytic review of the grit literature and found that grit had a very high correlation with conscientiousness $(\rho=.84)$. This finding raises the important question of whether these two constructs are empirically distinguishable. In addition, results reported by Muenks and her colleagues (2017) revealed that the two facets of grit exhibited significant overlaps with various constructs. Specifically, in their sample of university students, the perseverance of effort facet of grit was found to overlap most with self-control and conscientiousness ( $r$ 's $=.81$ and .77 , respectively); the consistency of interest facet of grit had the highest overlaps with effort regulation and behavioral engagement ( $r$ 's $=.97$ and .86 , respectively). These findings suggest that grit is likely to be indistinguishable empirically from these constructs.

Some studies have shown that grit is an important contributor to success and performance in academic and vocational settings. For instance, high levels of grit were found to be associated with attainment of higher education levels and fewer career changes (Duckworth et al., 2007). Similarly, university students enrolled at an elite university who had higher levels of grit were found to attain higher GPA than students who had lower levels of grit (Duckworth et al., 2007). Grittier competitors in the National Spelling Bee were inclined to spend more time in studying and practicing of spelling and advanced to further rounds in the final competition than the less gritty students (Duckworth et al., 2007; Duckworth \& Quinn, 2009). Grit has also predicted retention in academic and vocational settings. For example, gritty high school junior students were more likely to graduate in their 
senior year (Eskreis-Winkler, Shulman, Beal, \& Duckworth, 2014). Similarly, gritty novice teachers were more likely to stay in the teaching through the school year and had a better performance than those who were less gritty (Robertson-Kraft \& Duckworth, 2014).

However, other empirical findings are inconsistent with regard to the relationships between grit and academic achievement (Credé, 2018). For example, in their meta-analysis of grit literature, Credé and his colleagues (2017) reported that grit had a moderate positive association with overall academic performance $(\rho=.18)$. Muenks et al. (2017) showed that grit was not related to students' grades when personality, self-regulation, and engagement constructs were controlled for. In addition, some studies, which examined the two facets of grit separately, found either that (i) the perseverance of effort facet of grit was more strongly related to academic achievement than the consistency of interest facet; or that (ii) only perseverance of effort predicted achievement outcomes when controlling for some other demographic or motivation variables (Credé et al., 2017; Muenks et al., 2017; Muenks, Yang, \& Wigfield, 2018; Steinmayr et al., 2018).

In research, grit has been measured by the Grit Scale (Grit-O; Duckworth et al., 2007) and the Short Grit Scale (Grit-S; Duckworth \& Quinn, 2009). The Grit-O is a self-report measure developed by Duckworth et al. (2007), which comprises 6 items for perseverance of effort and 6 items for consistency of interest. Items are rated on a 5-point Likert-style scale from 1 (not at all like me) to 5 (very much like me). Higher scores are associated with higher levels of grit. Duckworth and Quinn (2009) further validated and refined the Grit-O, which resulted in the development of Grit-S, which consists of 4 items for perseverance of effort and 4 items for consistency for interest. Importantly, recent studies (e.g., Muenks et al., 2017; Muenks et al., 2018) argued that the current grit scales do not measure the long-term goals that are at the center of the conceptualization of grit. Specifically, in the Grit-S, only one item (i.e., "I have difficulty maintaining my focus on projects that take more than a few months to complete"; reverse scored) states a specific length of time; other items refer to a long-term goal only by using the word "later". In these researchers' views, these items do not capture well this important theoretical aspect of grit that distinguishes grit and other constructs such as conscientiousness and self-control (Muenks et al., 2018).

Although grit has received widespread attention, very little is known about 
motivational antecedents of grit (Eskreis-Winkler et al., 2014). Promotion, prevention, assessment, and locomotion are important motivation predispositions that shape what goals individuals set and how they pursue their goals (Higgins, 2012; Hodis, 2018a, 2018b). It is likely that these four motivation orientations influence the extent to which individuals are persistent when they face obstacles and maintain commitment to important long-term goals. For example, Mueller, Wolfe, and Syed (2017) reported that locomotion was a positive and strong predictor of grit $(\beta=.43)$, whilst assessment was a negative and moderately strong predictor of grit $(\beta=-.19)$. Similarly, the results reported by Pierro et al. (2011) showed that locomotion had positive relationships with perseverance of effort and consistency of interest. In contrast, assessment was found to be negatively associated with the two dimensions of grit; however, the correlation between assessment and perseverance of effort was not statistically significant (Pierro et al., 2011). Given these considerations, it is important to examine the relationships between the four orientations, on the one hand, and grit, on the other.

The perseverance of effort dimension of grit, which entails relentless pursuit of longterm goals (Duckworth et al., 2007; Duckworth \& Quinn, 2009), is likely to support the attainment of both promotion and prevention goals. Specifically, if a long-term goal involves advancing from a current status quo and making progress, being gritty in the process of attaining this goal sustains a promotion orientation. Moreover, promotion-focused individuals who are gritty are likely to strive eagerly to maximize goal-related gains. If a long-term goal involves maintaining the current status quo and guarding against any potential loss, being gritty in the pursuit of this goal sustains a prevention orientation. This is why, preventionfocused individuals who are gritty will vigilantly avoid making mistakes to ensure non-loss regarding their long-term prevention goals.

In contrast, the consistency of interest dimension of grit, which relates to maintaining (as opposed to frequently changing) goals or interests, is likely to be positively associated with a prevention rather than a promotion orientation. Prevention-focused individuals represent their goals as duties and responsibilities they must fulfil. The more valuable the goal is, the more committed they are to the goal (Higgins, 2012). Therefore, preventionfocused individuals are inclined to hold on to their original (important) goal or interest even 
in the face of obstacles and failures. In contrast, promotion-focused individuals strive to maximize gains; they do so by choosing goals that are high in both value and expectancy of success (Higgins, 2012). If they encounter obstacles that make success hard or unlikely, promotion-oriented individuals tend to abandon the original goal and strive for new goals of advancement (Higgins, 2012; Scholer \& Higgins, 2012). Therefore, promotion-focused individuals are likely to be less committed to the goal in the face of adversity (Higgins, 2012; Scholer \& Higgins, 2012). This is why, it is likely that promotion is negatively related to the consistency of interest facet of grit.

Locomotion is likely to be positively related to the two facets of grit. Both grit and locomotion emphasize making progress towards the goal. Locomotors tend to have a task orientation, that is, maintain control over attention to a task and persist conscientiously until the task is completed (Kruglanski et al., 2000). Therefore, it is likely that locomotion is positively related to the perseverance of effort facet of grit. In addition, individuals having high locomotion levels are likely to exhibit intrinsic motivations (Kruglanski et al., 2000). As a result, locomotors are engaged in what they are doing, resist distractions, and move toward their goal with focused attention (Higgins et al., 2003). Hence, locomotion is likely to be positively related to the consistency of interest facet of grit. These hypotheses are congruent with the results reported by Pierro et al. (2011). Specifically, the findings in Study 6 of this research showed that locomotion was a positive and strong predictor of the perseverance of effort dimension of grit $(\beta=.45)$ and a positive and moderately strong predictor of the consistency of interest facet of grit $(\beta=.22$; Pierro et al., 2011).

These aspects notwithstanding, there are some circumstances/situations in which locomotion may be negatively related to the consistency of interest facet of grit. Specifically, locomotion is primarily focused on movement and change, whereas grit entails consistency of goals even when progress is not apparent. Importantly, individuals with a strong locomotion orientation are more concerned with the likelihood of goal attainment than with the value outcomes associated with their movement (Higgins, 2012; Kruglanski et al., 2000). As a consequence, when obstacles or setbacks disrupt smooth movement, high locomotors are likely to be less committed to goals requiring them to overcome these obstacles. Moreover, locomotion is not necessarily concerned with movement toward any particular end-state, let 
alone a long-term goal (Cornwell et al., 2015). This is an additional reason to hypothesize that, in some circumstances, locomotion may be negatively associated with the consistency of interest dimension of grit. These aspects suggest that more research is needed to examine the relationships between locomotion and the consistency of interest facet of grit.

Assessment is likely to be negatively related to grit. Assessment is primarily concerned with comparisons and critical evaluations of alternative options in the service of identifying the best goal and/or the best means to attain the goal (Higgins, 2012). Hence, assessors are not committed to any goal until they feel that they know the truth about all alternatives (Cornwell et al., 2015). This is why, it is likely that assessment is negatively associated with the consistency of interest dimension of grit. Moreover, constant comparisons and critical evaluations could make assessors self-critical of the discrepancy between the present state and the desired state and, thus, have negative affect, low self-esteem and low optimism (Kruglanski et al., 2000). Hence, individuals having a strong assessment orientation tend to perceive achieving their goals as less likely (Higgins, 2012). This negative outlook could prevent them from working hard to attain a goal when encountering obstacles and failures. Therefore, it is likely that assessment is negatively related to the perseverance of effort facet of grit. The findings of Mueller et al. (2017) and Pierro et al. (2011) support this contention, as they showed negative relationships between assessment and grit/the two facets of grit.

In the following, I use the above-mentioned hypotheses to make predictions pertaining to grit in relation to distinct patterns of associations of the four orientations (i.e., motivation profiles) identified in recent research. In particular, I hypothesize which motivation profiles are likely to be more (vs. less) conducive to the two facets of grit. To do so, I use the weighted motivation profiles presented in Hodis (2018a), which summarize the motivation profiles of promotion, prevention, assessment, and locomotion identified in the existing literature. These weighted motivation profiles are $\mathrm{C} 1-\mathrm{C} 7$, outlined below.

The motivation profile underlying $\mathrm{C} 1$ is characterized by average and relatively comparable mean levels on all four orientations. Having average levels of promotion and prevention, these individuals are unlikely to be strongly motivated to work hard towards either ideal or ought goals. In addition, having average levels of locomotion, these individuals are less likely to maintain effort in the face of difficulties or obstacles. This is why these 
individuals may not display very strong perseverance of effort. At the same time, having average levels of prevention and locomotion, they are not very likely to be committed to a goal when meeting challenges. Therefore, these individuals may have moderate to low levels of consistency of interest.

The C2 profile includes low/very low levels of assessment, locomotion, and promotion and below average levels of prevention. Considering that all four orientations are weak, individuals having this type of motivation outlook are unlikely to be strongly motivated and devote effort to pursue any goal (Higgins, 2012; Hodis, 2018a). Thus, it is most likely that they give up effort when they encounter setbacks and are not strongly committed to any goal, let alone to a long-term goal. This is why, they may have low/very low levels of both facets of grit.

The C3 profile is characterized by low and comparable mean levels of assessment and locomotion, below average levels of promotion and above-average levels of prevention. In this profile, assessment and locomotion are likely to be too weak to play their self-regulatory roles. Therefore, average levels of promotion and prevention are likely to be the main determinants of motivation effects associated with this profile. These individuals are likely to have moderate to low levels of perseverance of effort. This is the case because average levels of promotion and prevention do not strongly motivate them to strive for ideal/ought goals; thus, they are less likely to devote extra effort when facing difficulties in their goal pursuits. Likewise, these individuals may not display strong consistency of interest because having average levels of prevention, they are not very likely to maintain an important goal for a long time.

The C4 profile is characterized by very strong assessment, locomotion, and promotion, as well as very weak prevention. In this profile, prevention would be too weak to contribute meaningfully to self-regulation. Thus, it is most likely that motivation effects associated with this motivational profile are shaped by the combination of strong assessment, locomotion, and promotion. Strong locomotion could constrain the self-regulatory drawbacks associated with strong assessment (e.g., excessively evaluating and comparing) and motivate these individuals to be perseverant the face of difficulties or setbacks. In addition, strong promotion may motivate them to eagerly strive for ideal goals. Taken together, these individuals are 
likely to have strong/very strong perseverance of effort. However, when facing obstacles, individuals who have a $\mathrm{C} 4$ type of motivation profile are likely to have relatively low consistency of interest. In this scenario, their dominant strong promotion may make them switch to other goals for advancement. At the same time, when these individuals experience little or no progress, their strong locomotion may motivate them to pursue other goals that could offer better opportunities for smooth progress (Higgins, 2012; Kruglanski et al., 2000). Therefore, these individuals are less likely to maintain commitment to original goals for a long time.

The C5 profile encompasses very high/high levels of assessment and locomotion, above-average levels of promotion, and very low levels of prevention. Within this profile, locomotion and promotion, which are comparable in magnitude, are somewhat stronger than assessment; prevention is too weak to play a meaningful self-regulatory role. Therefore, it is most likely that motivation effects associated with this motivational profile are shaped by high assessment and equally strong locomotion and promotion. Following the line of thought discussed in the previous paragraph, individuals who exhibit the C5 profile are likely to display relatively strong perseverance of effort and somewhat weak consistency of interest.

The C6 profile is characterized by average levels of assessment and strong/very strong levels of the remaining orientations. As they have average levels of assessment, individuals exhibiting this motivation profile may not spend a lot of time comparing and evaluating alternative goals and means. Therefore, motivation effects associated with this motivational profile are most likely to be shaped by a combination of strong locomotion, promotion, and prevention. As hypothesized above, promotion, locomotion, and prevention are likely to be positively associated with perseverance of effort. Hence, these individuals are likely to have strong/very strong perseverance of effort. Meanwhile, strong prevention could strengthen their commitment to important goals and constrain the tendency to switch goals in the face of obstacles, which are associated with strong promotion and locomotion. Hence, it is likely that the C6 profile is associated with high/very high levels of consistency of interest.

The C7 profile is characterized by strong assessment, below average levels of locomotion and promotion, and very weak prevention. Within this profile, assessment is stronger than the other three orientations and, therefore, it is most likely to shape motivation 
effects associated with this profile. As hypothesized above, assessment is likely to negatively relate to the two facets of grit. Therefore, it is possible that individuals who exhibit the C7 profile have low/very low levels of grit.

Theories of intelligence. Theories of intelligence (Dweck \& Leggett, 1988) have been an important construct in motivation research. These theories refer to two different beliefs individuals have about the nature of their intelligence. Specifically, an entity theory of intelligence comprises individuals' beliefs that intelligence is a fixed or static trait that cannot be changed throughout their lifetime. By contrast, an incremental theory of intelligence refers to individuals' beliefs that intelligence is malleable, and, thus, can be developed through effort (Dweck, 1999; Yeager \& Dweck, 2012). Endorsement of these theories motivates individuals in different ways. Importantly, when individuals hold an entity theory, they tend to try to validate their competence; in contrast, those holding an incremental theory seek learning opportunities to improve their abilities (Mathur, Chun, \& Maheswaran, 2016).

These two different beliefs are the core of individuals' self-systems and can affect individuals' motivation and academic performance (Dweck, 1999). For instance, theories of intelligence can determine the types of goals that individuals tend to pursue (Dweck, 1999; Dweck, Chiu, \& Hong, 1995; Dweck \& Leggett, 1988; Yeager \& Dweck, 2012). Specifically, individuals who hold an entity theory tend to pursue performance goals because they attempt to demonstrate their intelligence and gain positive judgments of their intelligence. In contrast, those who believe in an incremental theory of intelligence are likely to adopt learning or mastery goals because they try to learn new things and skills to improve their intelligence. Theories of intelligence also influence individuals' resilience in academic settings (Yeager \& Dweck, 2012). Specifically, these two beliefs about intelligence predict individuals' different responses to negative events and outcomes and strategies they adopt to deal with challenges or setbacks (Dweck, 1999; Hong, Chiu, Dweck, Lin, \& Wan, 1999). For example, individuals with an entity view of intelligence tend to ascribe success and failure to their inherent intelligence. Therefore, they view failure or negative feedback as a reflection of their intellectual inadequacy, are likely to show negative affect (e.g., helplessness), and tend to give up when facing challenges or setbacks (Hong et al., 1999). Conversely, individuals having an incremental view of intelligence attribute success and failure to their effort. Thus, 
they view failure and setbacks as indicators of insufficient efforts, are likely to exhibit positive affect, and tend to adopt mastery-orientated strategies (e.g., trying harder) when encountering setbacks or challenges (Hong et al., 1999). Existing research has provided consistent evidence that students' theories of intelligence affect their academic performance. In particular, incremental beliefs of intelligence were found to be associated with higher academic achievement than entity beliefs (Greene, Costa, Robertson, Pan, \& Deekens, 2010; Jones, Wilkins, Long, \& Wang, 2012).

Individuals' theories of intelligence can be measured by the Theories of Intelligence Scale (Dweck, 1999). The scale is composed of two subscales, namely, four entity theory statements (e.g., "You have a certain amount of intelligence, and you can't really do much to change it") and four incremental theory statements (e.g., "You can always substantially change how intelligent you are"). Items are rated on a 6-point Likert-style scale from 1 (strongly agree) to 6 (strongly disagree). Respondents indicate their degree of agreement or disagreement with each of the item statements. Higher final scores indicate an incremental theory of intelligence and lower final scores indicate an entity theory of intelligence.

An incremental theory of intelligence and a promotion orientation overlap in that both emphasize growth (Mathur et al., 2016). Individuals holding an incremental view of intelligence are concerned about learning and growth and regard challenges and setbacks as opportunities to learn and improve (Yeager \& Dweck, 2012). This incremental view sustains the promotion concerns with advancement and growth. Promotion-focused individuals strive to reduce the discrepancy between their actual and ideal selves (Higgins, 1997). Therefore, they are willing to expend effort to improve their weaknesses, persist in the face of obstacles and embrace challenges when there are opportunities for goal-related gains (Higgins, 2012). Therefore, it is likely that a promotion orientation is positively related to an incremental theory of intelligence.

There are some similarities between holding an entity theory of intelligence and having a prevention focus. Holding an entity belief of intelligence renders challenges, effort, and failure as indicators of lack of competence and, thus, "creates a psychological world of threats and defenses" (Yeager \& Dweck, 2012, p. 304). These perceptions of threat sustain vigilance, which is at the core of the prevention concerns with safety and security. 
Specifically, prevention-focused individuals tend to be vigilant against making mistakes to avoid potential losses and to maintain the status quo (Higgins, 1997). For example, they may avoid challenges that might threaten their motivation to validate their competence. Therefore, it is likely that a prevention orientation is positively related to an entity theory of intelligence. Notably, an entity theory of intelligence is not always identical to a prevention orientation. For instance, individuals holding an entity theory of intelligence may become helpless when encountering failure, while prevention-focused individuals are motivated to make more effort when facing or experiencing failure (Higgins, 2012).

An assessment orientation is associated with self-evaluative concerns; thus, high assessors are inclined to prove their competence and have better performance than others (Higgins et al., 2003). This tendency parallels those instigated by an entity theory of intelligence. Moreover, existing research suggests that high assessors are generally likely to believe that they cannot change the kind of person they are (Higgins et al., 2003). Individuals having high assessment levels also tend to have a critical perception of themselves due to their constant comparisons and evaluations of their performance (Higgins et al., 2003). These negative emotions could hinder their adaptive responses to negative feedbacks and failure. This is why, it is likely that assessment is positively related to an entity theory of intelligence.

Individuals holding an incremental theory of intelligence believe that intelligence can be improved through effort (Dweck, 1999). The malleability of intelligence involves the progress and change processes that are at the core of a locomotion orientation. Locomotors are likely to be motivated to make effort in learning and improve their competence because learning provides them with opportunities to progress (Higgins et al., 2003). In addition, their optimistic outlook will motivate them to respond to obstacles with persistence and vitality (Higgins et al., 2003). This is why, locomotion is likely to be positively related to an incremental theory of intelligence.

Critical thinking. Critical thinking is a cognitive process that involves deliberate and effortful reflection, analysis, and evaluation of extant evidence such as observation, experience, and communications with others (Byrnes \& Dunbar, 2014). Critical thinking also requires individuals to overcome their own biases in understanding and evaluating evidence and arguments (West, Toplak, \& Stanovich, 2008). While thinking critically, individuals tend 
to engage in deep processing of information and use complex cognitive strategies (Miele \& Wigfiled, 2014). Hence, critical thinking contributes to individuals' ability to collect and interpret information, acquire knowledge, and make sense of the world. Critical thinking is thus a desirable characteristic that students should develop in academic settings (Manalo, Kusumi, Koyasu, Michita, \& Tanaka, 2013).

Miele and Wigfiled (2014) proposed that critical thinking is an effortful form of information processing. According to these authors, when individuals think critically, they process information thoroughly and use complex cognitive strategies that involve high levels of effort and require cognitive engagement. Critical thinking can be characterized by a convergent (i.e., analytic) rather than divergent (i.e., associative) thinking mode (Miele \& Wigfiled, 2014). Convergent thinking is focused on finding the single best or correct answer to a problem by making logical connections between concepts. In contrast, divergent thinking is experienced as a low effort thinking, which involves generating multiple answers to a problem by combining and transforming ideas (e.g., brainstorming; Miele \& Wigfiled, 2014).

Engagement in critical thinking could serve a prevention orientation (Miele \& Wigfiled, 2014). According to regulatory focus theory (Higgins, 2012), the strategies individuals prefer to use to regulate their goal pursuits are shaped by their promotion or prevention orientations. More precisely, individuals having a strong promotion orientation tend to adopt associative (i.e., divergent) thinking (Miele \& Wigfiled, 2014). This is the case because, to maximize gains, promotion-focused individuals eagerly consider all available information, generate many ideas or hypotheses regarding a problem and prioritize speed over accuracy in information processing (Molden, 2012). These strategies are all consistent with divergent thinking. In contrast, prevention-focused individuals prefer analytical (i.e., convergent) thinking. This is the case because, to ensure against any potential losses, they try to vigilantly exclude incorrect information, narrow the options to find the best solution, and prioritize accuracy over speed in information processing to minimize errors (Molden, 2012). In turn, these strategies are all consistent with the convergent thinking that characterizes critical thinking. Taking all these aspects into consideration, it follows that critical thinking could be used in the service of a prevention orientation. In particular, during goal pursuits, the deliberate, careful and vigilant strategies that comprise critical thinking could help 
prevention-focused individuals to protect against losses (Miele \& Wigfiled, 2014).

According to the regulatory mode theory (Kruglanski et al., 2000), assessment involves comparing and evaluating alternative options to identify the best or correct choice. These comparisons and critical evaluations are essential attributes of critical thinking. Thus, individuals having high levels of assessment are likely to expend time and effort to engage in critical thinking, (e.g., weighing alternative options and synthesizing past and present experiences). Therefore, assessment is likely to be positively related to critical thinking.

Locomotion is likely to have a positive association with critical thinking. Locomotion has been found to be positively related to having a learning (i.e., mastery) orientation. Learning new knowledge or skills involves movement and change, which individuals high on locomotion are concerned with (Higgins et al., 2003). When they pursue a mastery goal, high locomotors are likely to engage in deep processing (e.g., relating what they are learning to previous knowledge, evaluating all available information sequentially and analytically), which characterizes critical thinking (Miele \& Wigfiled, 2014). This engagement could contribute to steady progress in knowledge and skill development, which fits a locomotion orientation. This is why, high locomotors are likely to use critical thinking.

The findings of Manalo et al. (2013) provide support for the afore-noted hypotheses regarding the relationships between regulatory mode orientations and critical thinking. More specifically, these authors reported that both assessment and locomotion were positively related to critical thinking; the association was weak for assessment and moderate for locomotion.

Effort regulation. Reeve and colleagues (2008) defined self-regulation as a process in which people organize and manage their capacities - that is, their thoughts (e.g., competence beliefs), emotions (e.g., interest), behaviors (e.g., engagement with learning activities), and socialcontextual surroundings (e.g., select a quiet, comfortable place to study) - in the service of attaining some desired future state (p. 223).

Self-regulation is a critical aspect of learning that is linked to positive outcomes in academic settings. For example, students who engage in effective self-regulation tend to have high levels of self-efficacy, be strongly interested in and value the learning tasks they work on, and 
have better academic performance (Pintrich \& De Groot, 1990; Yip, 2007; Zimmerman \& Schunk, 2008).

One important component of self-regulated learning is effort regulation (Pintrich \& De Groot, 1990). Pintrich and his colleagues defined effort regulation as individuals' capacity to exert effort and maintain attention when they face challenging or uninteresting tasks and other distractions in learning environments (Pintrich \& De Groot, 1990; Pintrich et al., 1991). Individuals who are effective at effort regulation are more likely to persist on a task until they achieve their study goal. By contrast, in similar situations, individuals who have weak effort regulation tend to quit before completing a task (Pintrich, 2004; Pintrich et al., 1991).

Effort regulation has been found to be associated with key motivation and personality factors (Komarraju \& Nadler, 2013; Pintrich, 1999; Richardson, Abraham, \& Bond, 2012; Sungur, 2007). For instance, effort regulation was positively related to self-efficacy and intrinsic value (i.e., viewing an academic task as intrinsically interesting and important; Pintrich \& De Groot, 1990) and with the Big Five personality traits of conscientiousness, intellect and agreeableness (Bidjerano \& Dai, 2007). Moreover, effort regulation was shown to be negatively associated with procrastination (Ziegler \& Opdenakker, 2018).

A large corpus of research has revealed that effort regulation is a key determinant of academic success (e.g., Credé \& Phillips; 2011; Komarraju \& Nadler, 2013; Pintrich \& De Groot, 1990; Richardson et al., 2012; Schwinger, Steinmayr, \& Spinath, 2009). For example, Pintrich and De Groot (1990) found that effort regulation positively related to middle school students' academic performance in actual classroom tasks and assignments (e.g., quizzes, tests, essays, and reports). Credé and Phillips (2011) conducted a meta-analysis that investigated the relationships between self-regulatory strategies and academic performance among university students. These authors reported that effort regulation was a robust positive predictor of both course grades and overall GPA. Notably, the effect sizes associated with effort regulation were found to be similar to those for some of the traditional influential predictors of academic achievement (e.g., grades on admission exams, previous academic performance, and study skills and study habits). Similarly, Muenks and colleagues (2017) also revealed that, for both high school and university students, effort regulation was a stronger positive predictor of students' grades than other important constructs such as grit, 
self-control, and conscientiousness.

Apart from being a predictor of academic success, effort regulation plays an important role as a link between motivation and academic achievement (Komarraju \& Nadler, 2013; Schwinger et al., 2009; Sungur, 2007). For instance, Komarraju and Nadler (2013) showed that, for university students, effort regulation mediated the relationship between self-efficacy and GPA. This finding suggests that students having strong self-efficacy are more likely to achieve better academic performance because they are more capable of maintaining their motivation and regulating their efforts in the face of challenges or distractions (Komarraju \& Nadler, 2013).

In research, effort regulation has been measured using four items from the effort regulation subscale of the Motivated Strategies for Learning Questionnaire (MSLQ; Pintrich et al., 1991). Sample items include "When course work is difficult, I gave up or only study the easy parts" (reverse scored) and "Even when course materials are dull and uninteresting, I manage to keep working until I finish". Items are usually rated on a 7-point Likert-style scale from 1 (not at all true of me) to 7 (very true of me). Higher scale scores indicate stronger effort regulation. This instrument has shown adequate reliability in previous empirical studies (Credé \& Phillips; 2011; Pintrich, Smith, Garcia, \& McKeachie, 1993; Richardson et al., 2012).

Promotion, prevention, assessment, and locomotion are basic self-regulatory orientations (Higgins, 2008). They play significant roles in shaping individuals' selfregulation of their goal pursuits (e.g., setting and selecting goals and adopting means to pursue goals; Higgins, 2012; Kruglanski et al., 2000). As noted above, effort regulation is an essential aspect of self-regulation. These four motivation orientations are likely to influence how individuals regulate their effort in the face of challenges or distractions. In this light, it is important to examine whether these motivation orientations are related to effort regulation in meaningful ways. Below I make predictions regarding the relationships between promotion, prevention, locomotion, and assessment, on the one hand, and effort regulation, on the other.

Promotion is likely to be negatively related to effort regulation. Specifically, a promotion focus motivates individuals to strive for gains and advancement. Individuals having a strong promotion focus are likely to be more engaged in achieving a new goal and 
less committed to maintaining a goal in the face of difficulties that make success unlikely (Higgins, 2012; Scholer \& Higgins, 2012). In turn, a lack of commitment associated with strong promotion could make people give up a difficult or boring task and switch to new tasks that facilitate advancement.

It is likely that prevention is positively associated with effort regulation. This is the case because a strong focus on fulfilling responsibilities, duties, and obligations could strengthen individuals' commitment to important goals (Higgins, 2012; Scholer \& Higgins, 2012). Therefore, prevention-oriented individuals tend to exert more effort on an important task and persist until they complete it even if they encounter difficulties or distractions.

Consistent with the tenets of the regulatory mode theory (Kruglanski et al., 2000; Higgins et al., 2003), locomotion is likely to be positively related to effort regulation. Specifically, individuals having strong locomotion tend to have a task orientation (i.e., attending to a task and persisting until completion; Kruglanski et al., 2000; Higgins et al., 2003). Moreover, locomotion is positively related to the intention to invest effort in goal pursuits (Pierro, Kruglanski, \& Higgins, 2006). Hence, when engaging in difficult or boring tasks, individuals high in locomotion are likely to devote more effort in what they are doing, resist distractions, and move toward their goal with focused attention. This is why, high locomotors are likely to have strong effort regulation.

Assessment is concerned with critical comparisons of alternatives and identifying the optimal one (Higgins, 2012; Kruglanski et al., 2000). In light of this proclivity, during task engagement, high assessors tend to stop to evaluate what they are doing, and thus, are less likely to maintain focus on the task at hand (Higgins et al., 2003; Pierro et al., 2006). Moreover, due to engaging in excessive comparisons and evaluations, high assessors tend not to be too optimistic with regard to achieving their goals (especially when they encounter obstacles) (Higgins et al., 2003). This negative outlook could prevent them from persisting on a challenging task. Therefore, these negative tendencies engendered by strong assessment could hamper effort regulation. This is why assessment is likely to be negatively related to effort regulation.

Below, based on the above-mentioned hypotheses, I make predictions with respect to effort regulation in relation to different patterns of interrelationships among the four 
orientations (i.e., motivation profiles) identified in recent research. Once again, I use the weighted motivation profiles presented in Hodis (2018a) to hypothesize which of these types of motivation profiles are likely to be more (vs. less) supportive of effort regulation.

As discussed previously, motivation effects associated with $\mathrm{C} 1$ are shaped by average and relatively comparable mean levels on all four motivation orientations. Having average levels of locomotion, these individuals are not likely to be very persistent when engaging in a difficult/uninteresting task. Similarly, having average levels of prevention, they may not be very motivated to fulfil responsibilities; therefore they are less likely to be committed to a goal when they encounter difficulties. Taken together, these individuals are not likely to have high levels of effort regulation.

Because individuals having the $\mathrm{C} 2$ profile have low/very low levels of assessment, locomotion, and promotion and below average levels of prevention, they are likely to be at risk for lack of motivation and disengagement from learning tasks (Higgins, 2012; Hodis, 2018a; Hodis et al., 2017). Therefore, they are likely to have (very) low effort regulation. Motivation effects associated with the $\mathrm{C} 3$ profile are most likely to be shaped by average and comparable levels of promotion and prevention. As hypothesized above, promotion is positively associated with a lack of commitment to original goals, and, therefore, is likely to motivate individuals to give up effort in face of difficulties (Higgins, 2012; Scholer \& Higgins, 2012). Having average levels of prevention, these individuals are not very likely to be motivated to fulfil obligations and duties; hence, they are not likely to be very committed to important goals. Taken together, these individuals may display relatively low levels of effort regulation.

Motivation effects associated with C4 are likely to be influenced by a combination of strong assessment, locomotion, and promotion. Strong locomotion could motivate these individuals to take action and stop them from excessive critical evaluations of goals and means (i.e., constrain excessive self-regulatory effects of high assessment; Higgins, 2012). At the same time, strong locomotion is likely to motivate these individuals to invest effort and maintain focus in their goal pursuits (Higgins et al., 2003; Pierro et al., 2006). This tendency could offset a lack of commitment associated with strong promotion. As a result, individuals having this profile are likely to persist until the completion of a task and, thus, display strong 
effort regulation.

Similar to the case of $\mathrm{C} 4$, high assessment and equally strong locomotion and promotion are critical determinants of motivation effects associated with the C5 motivation profile. As hypothesized previously, locomotion is likely to be positively associated with effort regulation. In addition, similar to the case of the $\mathrm{C} 4$ profile, strong locomotion can be effective in mitigating the potential negative effects that strong assessment and promotion may have on effort regulation. Hence, it is likely that these individuals display relatively strong effort regulation. Notably, as these three orientations are somewhat lower in C5 than in $\mathrm{C} 4$, it is likely that C5 is associated with lower levels of effort regulation than C4.

Motivation effects associated with C6 are most likely shaped by a combination of strong locomotion, promotion, and prevention. Both locomotion and prevention are likely to relate positively to effort regulation. Strong locomotion could engender tendencies that may weaken the potential negative association between promotion and effort regulation. Given these considerations, it is likely that the $\mathrm{C} 6$ profile is associated with average to high levels of effort regulation.

Within the $\mathrm{C} 7$ profile, as assessment is stronger than the remaining orientations; hence, motivation effects associated with this profile are most likely to be shaped by strong assessment. As is noted above, assessment is hypothesized to be negatively related to effort regulation. In this light, it follows that individuals exhibiting the $\mathrm{C} 7$ profile are likely to have low/very low levels of effort regulation.

Perceptions/evaluations of academic success. The construct of expectancy of success has been a focal one in the motivation literature (Eccles \& Wigfield, 2002; Gorges \& Göke; 2015; Hodis, 2018b). Expectancy of success is defined as an individual's belief about how successfully she/he will perform on a future task (Eccles \& Wigfield, 2002). Eccles and her colleagues (1983) differentiated between expectancy of success and other ability beliefs. Specifically, expectancy of success pertains to one's prospective assessment about the likelihood of success on a specific future task, whilst ability beliefs are individuals' evaluations of their current competence in different domains (Eccles \& Wigfield, 2002). Importantly, Eccles and Wigfield (2002) argued that in actual achievement settings, these constructs are empirically indistinguishable and can be used interchangeably. 
Empirical studies have shown that expectancy of success is "among the strongest psychological predictors of performance" (Wigfield et al., 2009, p. 59). For example, when students have higher expectancies of success, they devote more effort on a specific learning task, are more persistent in the face of difficulties, choose challenging courses/majors, and have better academic achievements (Conley, 2012; Eccles \& Wigfield, 2002; Perez, Cromley, \& Kaplan, 2014; Watt et al., 2012; Wigfield et al., 2015).

The expectancy-value model (Eccles \& Wigfield, 2002; Wigfield \& Eccles, 2000) posited that expectancies of success could be shaped by a set of individual and social factors (e.g., individuals' general motivation tendencies and their perceptions of socializers' expectations, beliefs, and attitudes towards them; Eccles \& Wigfield, 2002; Wigfield et al., 2009). Moreover, recent empirical studies explored the relationships between the key motivation orientations (i.e., promotion, prevention, locomotion, and assessment) on the one hand, and expectancy of success in mathematics, on the other (Hodis, 2018b; Hodis \& Hodis, 2015). The results of these studies revealed that promotion was a positive and strong predictor of expectancy of success; in contrast, the other three motivation orientations had generally weak or non-significant associations with expectancy of success (Hodis, 2018b; Hodis \& Hodis, 2015).

Perceptions of academic success examined in this study are similar to expectancies of success in the sense that they both are self-reported evaluations of success. However, the key difference between these two constructs is that expectancies of success are individuals' beliefs regarding the likelihood of attaining success in the future, whilst perceptions of academic success are students' evaluations of their academic achievements in the past years at university. No previous research has provided empirical evidence on the relationships between regulatory focus/mode orientations and individuals' evaluations of their previous academic success. As such, I chose to focus on perceptions of academic success to provide insight into the extent to which distinct motivation profiles may account for individual differences in this construct. Research has shown that self-reported academic achievements (e.g., school grades) are appropriate measures of students' academic achievements (Hennan, Dornbusch, Herron, \& Herting, 1997; Soenens \& Vansteenkiste, 2005; Taylor et al., 2014). Hence, in the following I overview briefly the literature on the relationships between the four 
motivation orientations (on the one hand) and actual academic achievements (on the other). This discussion provides a useful framework for investigating the interrelationships between perceptions/evaluations of academic success and regulatory focus/mode.

Rosenzweig and Miele (2016) examined the relationships between regulatory focus and students' performance in academic settings (e.g., in standardized tests and university course exams). Findings from this research suggest that prevention (vs. promotion) may play an adaptive role in students' performance in academic tests when there is minimal or no time pressure. Specifically, results reported by these authors indicated that when preventionfocused university students had adequate time to complete the academic tests, they scored higher on these tests than did their counterparts who had strong promotion orientations (Rosenzweig \& Miele, 2016). This study also found that in the math part of an academic test, prevention-focused students revisited more frequently the questions they had completed and answered more questions correctly than their promotion-focused counterparts (Rosenzweig \& Miele, 2016). Rosenzweig and Miele (2016) discussed that vigilant strategies (e.g., revisiting the previous questions) may be conducive to students' test performance. Specifically, individuals having a strong prevention orientation are motivated to ensure against any potential losses. Consequently, they prefer using vigilant strategies. When they have ample time, they are more likely to revisit the questions frequently to reduce the chances of making mistakes. In turn, this vigilance may contribute to better performance in an academic test promRosenzweig \& Miele, 2016).

Other empirical research explored the relationships between regulatory mode and individuals' actual academic achievements. For instance, Kruglanski et al. (2000; Study 7) reported that both locomotion and the interaction between locomotion and assessment had positive associations with grade point averages (GPA) at university $(\beta=.12$ and $\beta=.07$, respectively). Specifically, students having stronger locomotion were likely to have higher GPA than students with weaker locomotion. In addition, the effect of locomotion on GPA depended on the strength of assessment. In particular, strong locomotion predicted high GPA only when assessment was relatively high (Kruglanski et al., 2000). These findings suggest that optimal performance is supported by having high levels of both locomotion and assessment (Higgins, 2012; Kruglanski et al., 2000; Pierro et al., 2018). 


\section{Review of Empirical Research}

This section reviews the empirical studies on the four motivation orientations in Chinese/Asian contexts. First, it describes the literature search procedure. Then, it summarizes the empirical findings and identifies research gaps in the existing literature.

Literature search procedure. The search of the literature aimed to identify all studies that investigated the regulatory focus or regulatory mode of Chinese or Asian learners. The term "Chinese learner" has been used to refer to a subgroup of Asian learners from collectivist culture or Confucius Heritage Culture (Chan, 1999; Ho, Holmes, \& Cooper, 2004). Feng (2011) defined Chinese learners as those who either speak Mandarin Chinese "as their mother tongue or use it as an official language" (p. 2), including Chinese from Mainland China, Hong Kong, and Taiwan. However, this conceptualization of Chinese learners is problematic, because Chinese learners it defined come from a diverse geographic region with diverse Chinese cultures. For example, although people in Hong Kong are ethnic Chinese and share a lot of similarities in culture with mainland Chinese, the Hong Kong context has some differences in politics, economy, education and other cultural aspects from mainland China (Cheung, 2013). Hong Kong used to be the British colony and people in Hong Kong are more likely to have been exposed to Western cultural influences (Kurman \& Hui, 2012). Thus, these regional differences among Chinese learners should be noted.

This search was carried out through the Google Scholar search engine and four databases, namely, ProQuest, PsycINFO, Eric, and Education Source. The following search terms were used: ("regulatory focus" OR "regulatory mode"), (Chin* OR Asia* OR Hong Kong OR Taiwan), (student* OR learner* OR school* OR universit* OR college*), ("promotion" AND "prevention"), ("assessment" OR "locomotion"). Other inclusion/exclusion criteria (e.g., document type, language, and publication date) were also set to narrow the search results. Specifically, peer-reviewed original journal articles, book chapters, and theses were included; reports, book reviews, and news articles were excluded. Studies published from 1997 to 2018 were included because Higgins proposed the regulatory focus theory in 1997 and Kruglanski et al. put forward the regulatory mode theory in 2000.

Only articles, book chapters, and theses written in English were considered. Titles and 
abstracts in the search results were read to decide whether a piece of literature was relevant to this research and to what extent. With the use of the above mentioned search terms, 83 articles were found in the ProQuest database, 115 in PsycINFO, two articles in ERIC and 32 in Education Source. However, only nine articles were considered to be relevant to this research. The others needed to be excluded mainly because they were not based on the regulatory focus theory (Higgins, 1997) or the regulatory mode theory (Kruglanski et al., 2000 ), or because they investigated the regulatory focus or regulatory mode orientations in work settings rather than in an educational context (e.g., Akhtar \& Lee, 2014; Chen, Wen, \& Ye, 2017).

The citations of some key articles were also searched in order to source other relevant literature. For example, Google Scholar provided 5918 citations of Higgins (1997). Searching within these cited results for the search terms of China OR Chinese OR Asia OR Asians identified 1500 citations; however, only additional four articles were found to be relevant to this research. Similar search procedures were conducted with regards to Kruglanski et al. (2000). Among the 505 citations identified, two additional articles were found to be relevant to this research. In total, there were 15 articles concerning Chinese or Asian learners' regulatory focus or regulatory mode orientations.

Summary of empirical research findings. Recently, researchers have begun to explore regulatory foci and regulatory modes in the Chinese or Asian context (Kurman \& Hui, 2012; Lee, Aaker, \& Gardner, 2000; Liu \& Yao, 2018; Shu \& Lam, 2011, 2016; Zhang et al., 2015). One strand of research has examined the relationships between regulatory focus/regulatory mode and self-construal (Jin, Wang, \& Dong, 2016; Kurman, Liem, Ivancovsky, Morio, \& Lee, 2015; Lee et al., 2000; Lockwood, Marshall, \& Sadler, 2005; Manalo et al., 2013). Self-construal refers to individuals' perceptions of the relationships between the self and their environment (Markus \& Kitayama, 1991). Individuals having an independent self-construal regard themselves as independent and unique from others; in contrast, individuals having an interdependent self-construal put emphasis on the interdependence and harmony between themselves and others. The findings of Jin et al. (2016), Kurman et al. (2015) and Lockwood et al. (2005) showed that individuals from western and eastern cultures differed in their self-construal and regulatory focus. Specifically, 
individuals from Western (i.e., individual) cultures (e.g., U.S. Americans) tended to have an independent self-construal, which, in turn, was positively associated with a promotion focus. In contrast, individuals from East Asian (i.e., collectivist) cultures (e.g., Chinese) were more likely to have an interdependent self-construal, which positively related to a prevention focus. In addition, Lee et al. (2000) found that promotion vs. prevention focus could be primed by having chronically accessible or situationally activated independent vs. interdependent selfconstrual. For example, promotion-framed information was regarded as more important in comparison to prevention-framed information by the American participants who had independent self-construals. In contrast, prevention-framed information was considered as more important by the Chinese students who had interdependent self-construals (Lee et al., 2000). Manalo et al. (2013) focused on university students from Japan and New Zealand and examined the relationships between independent vs. interdependent self-construal and regulatory modes. These authors found that independent self-construal was positively related to locomotion; this association was of moderate magnitude. In contrast, interdependent selfconstrual was positively associated with assessment; this association was of weak to moderate strength.

Research conducted in this population has indicated that regulatory focus orientations could shape individuals' responses to success and failure (Kurman \& Hui, 2012; Lockwood et al., 2005; Shu \& Lam, 2011, 2016). For instance, Kurman and Hui (2012) found that people with different regulatory focus orientations used different strategies to cope with failure. In particular, participants with a high promotion focus (i.e., Israeli Jews and Hong Kong Chinese) preferred considering more potential solutions to deal with initial failure. In contrast, participants with a high prevention focus (i.e., Hong Kong Chinese and Israeli Arabs) persisted longer than those with low prevention (Kurman \& Hui, 2012). In a similar vein, Shu and Lam (2016) found that Hong Kong Chinese university students having a promotion focus were motivated by success feedback, while those exhibiting a prevention focus were motivated by failure feedback. Moreover, after receiving success feedback, promotion-focused students were found to perform better in a visual search test and to be more willing to make efforts to improve their visual search abilities than their preventionfocused counterparts. By contrast, after receiving failure feedback, prevention-focused 
students had a better performance in the test and displayed more willingness to make efforts to improve their visual search abilities than promotion-focused participants.

Some other studies examined the effect of regulatory focus on achievement-related behaviors such as creativity (Jin et al., 2016; Kurman et al., 2015; Lam \& Chiu, 2002). For example, Kurman et al. (2015) investigated the extent to which regulatory focus accounted for cultural differences between Jewish and Japanese university students in divergent thinking, which is an antecedent of creativity. Their findings indicated that promotion focus was positively related to divergent thinking, while prevention negatively predicted divergent thinking. This pattern of interrelationships may undergird the fact that the Japanese sample, which exhibited low promotion and high prevention, also had low levels of divergent thinking (Kurman et al., 2015). Consistent with this hypothesis, Lam and Chiu (2002) found that Hong Kong Chinese undergraduate students having high promotion focus generated more ideas in a creative task than their counterparts having low promotion focus; in contrast, prevention focus was not related to the number of ideas generated.

Other studies investigated regulatory focus in academic settings (Kurman et al., 2015; Li \& Wu, 2011; Zhang, 2016). Kurman et al. (2015) explored the relationships between regulatory focus and mastery goal of Swiss, Mexican, and Indonesian university students. These authors found that students' promotion (prevention) focus was a positive (negative) predictor of mastery orientation. $\mathrm{Li}$ and $\mathrm{Wu}$ (2011) examined regulatory focus and optimism of university students in Taiwan. They found that students with a high promotion level scored significantly higher on optimism than did students with a low promotion level; in contrast, students with a high prevention level were significantly less optimistic than students with a low prevention level.

Another strand of the existing literature examined the motivation configurations (i.e., profiles) that emerge when investigating the two regulatory focus orientations. For instance, Liu and Yao (2018) identified four distinct regulatory focus profiles for a sample of Chinese preadolescents and adolescents. Specifically, the first of these profiles, which they named the "High regulatory focus" profile, was characterized by high levels of both promotion and prevention. The second profile, named the "Moderate regulatory focus" profile, was characterized by moderately high and comparable levels of promotion and prevention. The 
third profile, titled the "Low regulatory focus" profile, encompassed low levels of both promotion and prevention. The fourth profile, namely the "Primarily promotion-oriented" profile, was characterized by strong promotion and weak prevention.

In addition, Liu and Yao (2018) examined the relationships between the Big-Five personality traits (Barrick \& Mount 1991; Costa \& McCrae, 1992) and the regulatory focus profiles identified in their study. The findings showed that participants who reported higher conscientiousness and openness were more likely to exhibit either the motivation profile that was characterized by strong promotion and weak prevention or the profile where both promotion and prevention were strong. Participants who reported higher neuroticism were more likely to display the motivation outlook that was characterized by high levels on both orientations.

Moreover, the findings in this study revealed that there were similarities/differences among the motivation profiles uncovered with regard to psychological outcomes (i.e., selfesteem, loneliness, and life satisfaction). Specifically, the "Primarily promotion-oriented" profile was associated with the strongest self-esteem and life satisfaction and weakest loneliness among the four profiles. In contrast, the "Low regulatory focus" profile was related to the lowest self-esteem and life satisfaction and the highest loneliness. The "High regulatory focus" profile was associated with similar levels of self-esteem and loneliness to, and somewhat stronger life satisfaction than, the "Moderate regulatory focus" profile.

Very few studies have examined the regulatory mode of Chinese or other Asian students (for exceptions see Hong, Tan, \& Chang, 2004; Manalo et al., 2013; Zhang et al., 2015). Hong et al. (2004) investigated the relationships between regulatory modes and subjective well-being of ethnically Chinese undergraduate students in Singapore. These authors found that subjective well-being was lowest when individuals were high on assessment and low on locomotion but highest when individuals were low on assessment and high on locomotion. Zhang et al. (2015) explored the role of regulatory mode in academic engagement and academic burnout of university students in mainland China. They found that locomotion was positively associated with academic engagement and negatively with academic burnout; the association was of strong magnitude for academic engagement and of moderate strength for academic burnout. In contrast, assessment was positively related to 
academic burnout and negatively to academic engagement; these associations were both of moderate magnitude (Zhang et al., 2015). Manalo et al. (2013) investigated the relationships between regulatory mode and critical thinking of university students from two cities in Japan and from New Zealand. Their findings indicated that both locomotion and assessment were positively related to critical thinking in all samples investigated; this association was of moderate strength for locomotion and weak for assessment.

Research gap. There is a dearth of research concerning regulatory focus and regulatory mode in Chinese or Asian contexts. There were only 15 relevant articles concerning Chinese or other Asian learners' regulatory focus or regulatory mode among the 5918 citations Google Scholar provided for Higgins (1997) and the 505 citations it provided for Kruglanski et al. (2000). Moreover, the existing literature rarely examined interactions even between any two of the four key orientations that are at the center of this research (i.e., promotion, prevention, assessment, and locomotion), let alone among all the four orientations. This is an important limitation given Higgins' (2012) theorizing that promotion and prevention value work together with assessment (i.e., truth) and locomotion (i.e., control) to shape self-regulation and motivation effects. Thus, the theory suggests that none of these four orientations exerts isolated effects on motivation; on the contrary, all motivation effects are shaped by the interactions among the four orientations. To overcome this limitation and advance understanding of the antecedents of motivation effects, research needs to identify the motivation profiles (i.e., the distinct and motivationally relevant combinations of promotion, prevention, assessment, and locomotion) that characterize individuals in this population.

Another limitation of the present literature concerns the fact that for most relevant studies identified, participants who provided data were recruited outside mainland China. Among the eleven articles concerning Chinese learners' regulatory focus and regulatory mode orientations, only four (i.e., Jin et al., 2016; Liu \& Yao, 2018; Zhang et al., 2015; Zhang, 2016) collected data from participants from mainland China. The other studies mentioned above involved Chinese or ethically Chinese students in Hong Kong, Taiwan, and Singapore. As discussed earlier, it should be noted that there might be some regional differences within the Chinese society. More specifically, students' motivations may differ due to economic, political, and other contextual factors that vary across geographical areas (Chen \& Wong, 
2015). Thus, to bridge the knowledge gap, research needs to examine specific motivation profiles that characterize mainland Chinese students and to explore the extent to which interindividual differences in key motivation factors/effects (e.g., high vs. low levels of grit, high vs. low effort regulation) are associated with distinct motivational profiles.

A final important limitation of the literature pertaining to regulatory focus and regulatory mode in Chinese or Asian contexts is that all existing relevant studies adopted quantitative methodologies or used experimental designs. As a consequence, most of these studies measured the constructs of regulatory focus and regulatory mode by means of selfreports. For instance, the Regulatory Focus Questionnaire (Higgins et al., 2001) was used by Jin et al. (2016), Kurman and Hui (2012), and Lam and Chiu (2002). The General Regulatory Focus Measure (Lockwood, Jordan, \& Kunda, 2002) was used by Kurman et al. (2015), Li and Wu (2011), Liu and Yao (2018), and Lockwood et al. (2005). The Regulatory Mode Scales (Kruglanski et al., 2000) were employed by Hong et al. (2004), Manalo et al. (2013) and Zhang et al. (2015). In some other studies (Lam \& Chiu, 2002; Shu \& Lam, 2016), the constructs of regulatory focus were manipulated experimentally (e.g., by priming). Although quantitative methods have important strengths (e.g., generating representative data, collecting a large quantity of data within a relatively short period of time; Robson, 2011), collecting data by means of surveys or questionnaires is not without limitations. For example, participants might misunderstand a question and provide an irrelevant response, which, in turn, might weaken the validity of data (Kelle, 2006).

Creswell and Plano Clark (2011) propose that a mixed-methods approach (i.e., the combination of qualitative and quantitative approaches) can provide benefits that complement the weaknesses of either approach and contribute to a better understanding of a research problem. For instance, this approach enables the researcher to obtain access to multiple sources of data by using all the available tools of data collection (e.g., questionnaires, interviews). Moreover, the researcher could triangulate the multiple data to arrive at conclusions with more confidence (Creswell \& Plano Clark, 2011). By using a mixed-method framework for this research, the quantitative phase could enable the researcher to obtain data from a larger number of individuals and identify participants' motivation profiles. In addition, this phase could help evaluate similarities and differences among profiles with regard to 
important motivation factors (i.e., grit, theory of intelligence, critical thinking, effort regulation, and perceptions/evaluations of academic success). In addition, quantitative data could allow the purposive selection of a particular group of participants for the qualitative phase. Furthermore, the existing literature focuses on aspects associated with having high (vs. low) levels of promotion, prevention, assessment and locomotion (Harring \& Hodis, 2016). As a result, current knowledge cannot be used to predict motivation effects/differences for participants whose motivation profile is characterized by average and comparable levels of promotion, prevention, assessment, and locomotion. Therefore, obtaining qualitative data on the motivation of these individuals would be helpful to understand motivation effects.

Qualitative designs enable researchers to explore in more details individuals' choices and behaviors (Johnson \& Christensen, 2014). Therefore, the qualitative approach, which was used in the second phase of this research, could help advance understanding of how regulatory focus and regulatory mode orientations interact to shape important motivation factors/effects. For example, qualitative analyses could provide an in-depth understanding of different approaches to learning that particular students exhibiting distinct motivation profiles report (e.g., being motivated by success vs. failure, using strategies that prioritize speed vs. accuracy).

\section{Chapter Summary}

In this chapter, I overviewed the conceptual and substantive literature that is relevant to the study. First, I outlined Higgins' (2012) conceptualization of motivation, regulatory focus theory (Higgins, 1997, 2012), and regulatory mode theory (Kruglanski et al., 2000). Then, I introduced the key motivation orientations (i.e., promotion, prevention, assessment, and locomotion) investigated in the research. Moreover, I highlighted potential relationships between these motivation orientations and important motivation factors/outcomes in learning settings. In the final section of the chapter, I reviewed the empirical studies that investigated the regulatory focus/mode orientations of Chinese/Asian learner and identified the research gaps. In the next chapter, I will describe the methodology adopted in this study. 


\section{Chapter Three Methodology}

Having reviewed the relevant literature and identified the potential gaps, this chapter focuses on the methodology. A mixed methods approach was adopted in this research. Mixed methods research requires that the researcher collect, analyse, and integrate both qualitative and quantitative data in a single study or in multiple phases of a programme of research (Creswell \& Plano Clark, 2011) to contribute to a more comprehensive understanding of the research questions than either method in itself (Creswell, 2014).

Within the chapter, I first introduce the research aim and research questions. Then, I justify the use of multiple paradigms, which underpin the mixed-methods design in this research, and provide an overview of the research design. I also present the details of each phase of the research with its particular participants, instruments, methods and procedures of data collection and data analysis. Lastly, I discuss the validity and trustworthiness of this mixed-methods research and ethical considerations.

\section{Research Aim and Research Questions}

This research aimed to (i) identify the motivation profiles that map the interrelationships among promotion, prevention, assessment, and locomotion in a sample of undergraduate students from mainland Chinese, (ii) to evaluate whether there are significant similarities/differences among the profiles uncovered with respect to a set of motivation factors that influence students' learning and academic performance, and (iii) examine the extent to which students' reported approaches to learning are consistent with the characteristics of their motivation profiles.

The following research questions were addressed:

1. How many motivation profiles account parsimoniously for variability in the interrelationships among the four motivation orientations in a sample of mainland Chinese undergraduate students?

2. Are there differences among the motivation profiles identified in this research with regard to students' levels of grit?

3. Are there differences among the motivation profiles identified in this research 
with regard to students' theories of intelligence?

4. Are there differences among the motivation profiles identified in this research with regard to students' use of critical thinking?

5. Are there differences among the motivation profiles identified in this research with regard to students' effort regulation?

6. Are there differences among the motivation profiles identified in this research with regard to students' perceptions/evaluations of academic success?

7. How do approaches to study reported by the participants align with their motivation profiles?

\section{Research Paradigms}

Researchers bring their philosophical assumptions to their studies, which influence what questions they choose to address and how they conduct their research (Creswell \& Plano Clark, 2011; Morgan, 2007). These assumptions are paradigms or worldviews. Creswell (2008) defined paradigms as "a general orientation about the world and the nature of research that a researcher holds" (p. 6). This orientation could "influence the kinds of knowledge researchers seek and how they interpret the evidence they collect" (Morgan, 2007, p. 50).

The selection of a paradigmatic stance is shaped by the researcher's beliefs and research experiences (Creswell, 2008). This study derived from my belief as a researcher that students' motivation is a complex phenomenon. Motivation can be measured by using quantitative scales. However, motivation-related beliefs and values exist cognitively and, thus, are hard to access directly. Therefore, multiple methods and research instruments were needed to capture students' motivation and its effects. This approach necessitated a mixed methods design incorporating multiple paradigms (Creswell \& Plano Clark, 2011). Tashakkori and Creswell (2007) defined mixed methods research as "research in which the investigator collects and analyses data, integrates findings, and draws inferences using both qualitative and quantitative approaches or methods in a single study or a programme of inquiry" (p.4). 
Each of the two distinct approaches within a study reflects a different way of seeing, understanding, and valuing the world (Creswell \& Plano Clark, 2011). First, a quantitative approach (e.g., using questionnaires based on theories) was adopted within a post positivist paradigm, which assumes that the world is governed by laws and theories. Within this framework, a researcher can test or verify these laws and theories through scientific methods (e.g., measurement of the objective reality; Creswell, 2014). This quantitative approach allowed me to identify different motivation profiles and evaluate the similarities and differences among the profiles based on data provided by a large sample. To do so, I employed validated instruments and used appropriate statistical procedures. Following this, qualitative interviews were conducted within a constructivist paradigm. Adopting this stance, my role as a researcher was to understand and interpret participants' perceptions of their experiences and value their subjective knowledge (Creswell, 2014). A constructive perspective could balance the information in the quantitative phase by exploring the subjective meanings of motivation of the participants. Embracing the two distinct paradigms allowed me to be open to different research methods and forms of data collection and analysis.

\section{Research Design}

This research used the mixed methods explanatory sequential design (Creswell, 2014). This research design comprised two distinct phases, with a quantitative study followed up by a qualitative study (i.e., QUAN $\rightarrow$ qual; Creswell \& Plano Clark, 2011). The conceptual map of the research design is illustrated in Figure 4. Specifically, I first collected and analyzed the quantitative data provided by the questionnaires. Five distinct motivation profiles were identified that mapped the interrelationships among the motivation orientations. Then, I conducted a multiple-case study by collecting and analyzing data from five cases (Stake, 1995; Yin, 2009); each case consisted of interview participants having a similar motivation profile identified in the research. I collected qualitative data through semi-structured interviews and analyzed these data to further explain the quantitative findings from the first phrase. The quantitative and qualitative phases were linked when selecting participants for 
the qualitative interviews. Specifically, I selected three to five participants from each motivation profile identified in the first phase. This sequential sampling enabled me to obtain "complementary databases that include information that has both depth and breadth regarding the phenomenon under study" (Teddlie \& Yu, 2007, p. 85). The findings of the quantitative and qualitative phases were integrated in the discussion section.

Lochmiller and Lester (2015) explained that the mixed methods research "draws upon the strengths of qualitative and quantitative research methods to wholly describe and study the research topic, phenomenon, or experience" (p. 450). In addition, combining numerical and in-depth data could increase credibility of research findings (Hesse-Biber, 2010). 


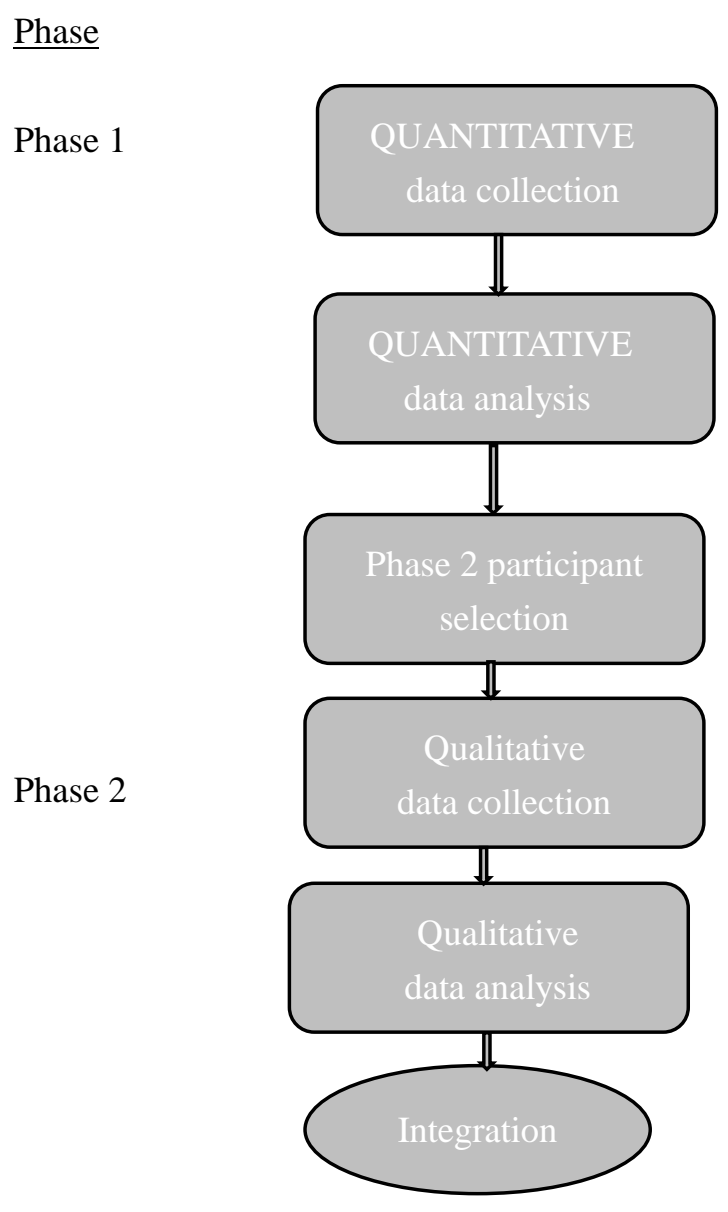

$\underline{\text { Research questions }}$

- $\quad$ Questions 1-6

$\underline{\text { Procedure }}$

- Administering of questionnaires

$>$ Paper survey in mainland China

$$
(N=886)
$$

- Confirmatory factor analysis (CFA)

- Latent profile analysis (LPA)

- Comparing motivation profiles on eight criteria of interest

- Purposeful sampling for Phase 2

- Question 7

- Multiple-case study:

$>$ Semi-structured interviews

- Coding and thematic analysis

- Integrating quantitative and qualitative findings
Product

- Numerical data

- Types of motivation profile

- Similarities and differences among profiles with regard to the key motivation factors/outcomes

- Number of interviewees (35 for each profile)

- Discourse data

- Codes, categories, and themes

- Discussion

- Implications

- Limitations

Figure 4. The mixed methods explanatory sequential design procedures. Adapted from Designing and conducting mixed method research (2nd ed.), by J. W. Creswell, and V. L. Plano Clark, 2011, p. 121. 


\section{Phase One: Quantitative Data Collection and Analysis}

This section discusses the participants, the quantitative instruments, data collection, and data analysis in the quantitative phase.

Participants. Convenience sampling was adopted to recruit participants. I selected one public provincial university in my home city, a provincial capital city of mainland China. The target university has a wide range of academic disciplines, such as economics, management, literature, law, science, engineering, education, and fine arts. It boasts 61 four-year undergraduate programmes and enrolls approximately 31,000 full-time undergraduate students located on four campuses. Participants were recruited from eligible undergraduate students from seven schools (i.e., Computer Science and Technology, Finance, Foreign Studies, International Education, International Trade and Economics, Management Science and Engineering, and Public Administration and Policy) in this university.

During April and May 2017, I first sought approval from the heads of schools in the university to contact lecturers who were teaching undergraduate students. After gaining approval, I approached the lecturers by phone and asked for permission to visit their classes and distribute information sheets (See Appendix A) to their students. I then distributed the questionnaires in class to the students who agreed to participate in the research.

Table 3 summarizes the demographic characteristics of the participants including gender, years at university, and major. As the table indicates, more females $(610 ; 68.8 \%)$ than males $(265 ; 29.9 \%)$ participated in completing the questionnaires, whereas five participants $(0.6 \%)$ chose "other" and six students $(0.7 \%)$ did not provide information on their gender. The gender imbalance in the sample was due to the high proportion of female students in the schools where I recruited participants. In addition, the majority of the participants were in the second and third years. Specifically, 30.4\% $(N=269)$ of the sample spent less than one year in a degree programme, $34.2 \%(N=303)$ between one and two years, and 35.4\% $(N=314)$ between two and three years. Finally, the largest group of participants were enrolled in undergraduate programmes of International Commerce and Economics. 
Table 3

Demographic Information of Questionnaire Participants

\begin{tabular}{lllc}
\hline Characteristics & Category & Number & $\begin{array}{c}\% \text { Total } \\
N=886\end{array}$ \\
\hline Gender & Female & 610 & 68.8 \\
& Male & 265 & 29.9 \\
& Other & 5 & 0.6 \\
& Not disclosing gender & 6 & 0.7 \\
Years at university & Less than 1 year & 269 & 30.4 \\
& 1-2 years & 303 & 34.2 \\
2-3 years & 314 & 35.4 \\
& Accounting (Sino-New Zealand) & 46 & 5.2 \\
& Business English & 69 & 7.8 \\
& Computer Science and Technology & 133 & 15.0 \\
& Electronic Commerce & 75 & 8.5 \\
& Financial Engineering & 23 & 2.6 \\
& Information Management & 38 & 4.3 \\
& International Business & 50 & 5.6 \\
& International Commerce and & 197 & 22.2 \\
& Economics & & \\
& Internet Engineering & 37 & 4.2 \\
Investment & 17 & 1.9 \\
& Labour and Social Security & 92 & 10.4 \\
Teaching Chinese to Speakers of & 109 & 12.3 \\
& Other Languages & & \\
\hline
\end{tabular}

Quantitative instruments. For this phase of the research, questionnaires were used to collect data from a large sample (Merten, 2006; Robson, 2011) within a relatively short period of time efficiently and economically (Creswell, 2014; Johnson \& Christensen, 2017). At the same time, this method of data collection ensured anonymity and confidentiality for participants, this allowing them to answer questions with frankness (Robson, 2011).

Six validated scales were used to collect data. These instruments are summarized in Table 4. All of these instruments have been found to measure their respective constructs in reliable and valid ways across studies (e.g., Higgins et al., 2001; Hodis, 2015, 2018b; Hodis et al., 2017; Hodis \& Hodis, 2017; Kruglanski et al., 2000). Moreover, previous research using Chinese samples show that Regulatory Focus Questionnaire (RFQ; Higgins et al., 2001) and Regulatory Mode Questionnaire (RMQ; Kruglanski et al., 2000) had good reliability. For instance, Kurman and Hui (2012) reported that Cronbach's alpha for the 
promotion items was 0.67 and the Cronbach's alpha for the prevention items was 0.73 . In the study conducted by Zhang and his colleagues (2015), the Cronbach's alpha was 0.70 for the locomotion items and 0.72 for the assessment items. In this study, the internal consistency of the set of items used to gauge promotion, prevention, assessment, locomotion, and other motivation constructs (measured by Cronbach's Alpha) is reported in Table 4.

The questionnaire was administered in English. All participants were learning English as part of their degree programmes. To make the items more understandable to the participants, I made some minor changes to the original wording of some items. For example, I rewrote the negative wording (e.g., "I don't spend much time") by using their positive equivalents (i.e., "I spend a lot of time"). I also rewrote the questions as statements for greater clarity. For example, I replaced "Growing up, would you ever 'cross the line' by doing things that your parents would not tolerate?" with "Growing up, I often 'crossed the line' by doing things that my parents would not tolerate". The modifications to the original wording were reviewed by my supervisors. Further information about the modifications is shown in Appendix B. Previous research (e.g., Hodis, 2018b; Hodis et al., 2017; Hodis \& Hodis, 2017) showed that these minor alterations were unlikely to influence the conceptual meaning of the original items; in addition, after slightly modifying the original wording, the instrument measured the constructs in a valid and reliable way.

The questionnaire comprised three parts (see Appendix C). To assist the participants to clearly understand what to do, clear and concise instructions in both English and Chinese were provided for each section (Creswell, 2014). Part one of the questionnaire included 60 items, which were recorded on a 7-point Likert-style scale ranging from 1 (strongly disagree) to 7 (strongly agree). Using the Likert scale enabled the respondents to clearly indicate their levels of agreement with each item (Johnson \& Christensen, 2017). Part two captured participants' demographic information such as gender, years at university, major, and selfevaluation of their academic success at university, which was rated on a 1-100 scale. Part three consisted of three puzzle questions based on the Cognitive Reflection Test (Frederick, 2005) to obtain the participants' actual performance data with respect to critical thinking. At the end of the questionnaire, participants were given the opportunity to record their contact details such as personal email addresses and QQ or Wechat (social media APPs) numbers if 
they were interested in being contacted about participating in a follow-up individual interview. 
Table 4

Description of the Measures Used in the Quantitative Phase

\begin{tabular}{|c|c|c|c|c|}
\hline Construct & $\begin{array}{l}\text { Number } \\
\text { of items }\end{array}$ & Instrument & $\begin{array}{l}\text { Cronbach's } \\
\text { Alpha }\end{array}$ & Examples of Items \\
\hline Promotion & 6 & $\begin{array}{l}\text { Regulatory Focus Questionnaire (RFQ; } \\
\text { Higgins et al., 2001) }\end{array}$ & 0.663 & Accomplishing things motivates me to work even harder. \\
\hline Prevention & 5 & RFQ (Higgins et al., 2001) & 0.842 & $\begin{array}{l}\text { I usually obeyed rules and regulations that were } \\
\text { established by my parents. }\end{array}$ \\
\hline Assessment & 12 & $\begin{array}{l}\text { Regulatory Mode Questionnaire (RMQ; } \\
\text { Kruglanski et al., 2000) }\end{array}$ & 0.773 & I often compare myself with other people. \\
\hline Locomotion & 12 & RMQ (Kruglanski et al., 2000) & 0.791 & When I decide to do something, I can't wait to get started. \\
\hline $\begin{array}{l}\text { Grit- Perseverance } \\
\text { of effort }\end{array}$ & 4 & $\begin{array}{l}\text { Short Grit Scale (Grit-S; Duckworth \& } \\
\text { Quinn, 2009) }\end{array}$ & 0.629 & I am a hard worker. \\
\hline $\begin{array}{l}\text { Grit-Consistency } \\
\text { of interest }\end{array}$ & 4 & Grit-S (Duckworth \& Quinn, 2009) & 0.585 & $\begin{array}{l}\text { I often set a goal but later choose to pursue a different one } \\
\text { (reverse scored). }\end{array}$ \\
\hline $\begin{array}{l}\text { Entity theory of } \\
\text { intelligence }\end{array}$ & 4 & $\begin{array}{l}\text { Theories of Intelligence Scale (Dweck, } \\
\text { 1999) }\end{array}$ & 0.899 & $\begin{array}{l}\text { I have a certain amount of intelligence, and I can't really } \\
\text { do much to change it. }\end{array}$ \\
\hline $\begin{array}{l}\text { Incremental theory } \\
\text { of intelligence }\end{array}$ & 4 & $\begin{array}{l}\text { Theories of Intelligence Scale (Dweck, } \\
\text { 1999) }\end{array}$ & 0.899 & I can always substantially change how intelligent I am. \\
\hline Critical thinking & 5 & $\begin{array}{l}\text { Critical Thinking Scale of the Motivated } \\
\text { Strategies for Learning Questionnaire } \\
\text { (MSLQ; Pintrich et al., 1991) }\end{array}$ & 0.741 & $\begin{array}{l}\text { I treat the course material as a starting point and try to } \\
\text { develop my own ideas about it. }\end{array}$ \\
\hline Effort regulation & 4 & $\begin{array}{l}\text { The Effort Regulation Scale of MSLQ } \\
\text { (Pintrich et al., 1991) }\end{array}$ & 0.646 & $\begin{array}{l}\text { Even when course materials are dull and uninteresting, I } \\
\text { manage to keep working until I finish. }\end{array}$ \\
\hline Total items & 60 & & & \\
\hline
\end{tabular}


Data collection. The questionnaire was pilot tested with a group of Chinese undergraduate students. Subsequently, I administered in person the questionnaires in class.

Pilot study. I pilot tested the questionnaire with nine Chinese undergraduate students whose levels of English proficiency were similar to those of the potential participants. These students were excluded from the final quantitative sample. I emailed the draft questionnaires to the pilot participants and asked them to provide feedback on the clarity of the questionnaire items. Some participants reported that they were not sure of the meanings of several words in the questionnaire items (e.g., "go-getter"). Based on their feedback, I refined the questionnaire by adding Chinese translations in brackets to the words that might be unclear to Chinese students (See Appendix C).

Administration of questionnaires. To achieve a satisfactory response rate, I consulted with several lecturers at the target university about the most effective way of administering the questionnaires. They recommended using paper questionnaires, for the internet was not readily available on some campuses and students did not regularly check their emails for notices.

The administration of the questionnaires took place between May and June 2017. I visited 25 classes on four campuses and briefed the students on the nature of the research. Participation was voluntary, with no incentives offered. I administered the questionnaires during regular class sessions. My presence in the classrooms allowed me to answer questions concerning instructions and questionnaire items raised by the participants (Cohen, Manion, \& Morrison, 2007). Participants appeared interested in completing the questionnaires and spent 20 to 30 minutes doing so. Finally, 886 valid questionnaires were collected. Data obtained from the questionnaires were entered manually into SPSS for data analysis.

Data analysis. Quantitative data from the questionnaires were analyzed using confirmatory factor analysis (CFA) and latent profile analysis (LPA).

$\boldsymbol{C F A}$. CFA is one of the most widely used modeling approaches to investigate the hypothesized relationships between a particular construct and its measured variables (Brown, 2006; Byrne, 2011). In social sciences, hypothetical constructs (e.g., motivation orientations) are latent variables or factors that cannot be observed and measured directly. Hence, researchers can only gauge some observed variables (e.g., questionnaire items) that serve as indicators of a latent factor. Interpretation of any latent variable (construct) is given by the fact that "an individual's standing on this unobserved dimension can be indicated by various proxies of the dimension" (Raykov \& Marcoulides, 2006, p. 116), which are called indicators. Given that indicators "are directly measureable manifestations of the underlying 
latent dimension" (Raykov \& Marcoulides, 2006, p. 116), CFA is often used to evaluate the extent to which a given set of indicators provides a valid and reliable measurement of corresponding latent factor(s) in a target population (Brown, 2006; Byrne, 2011; Geiser, 2013). An important benefit of measuring latent variables by means of indicators is that it "tends to reduce the effect of measurement error in any individual indicator on the accuracy of the results" (Kline, 2005, p. 165).

The key steps of CFA include model specification, identification, estimation, and evaluation (Brown, 2006; Wang \& Wang, 2012). CFA is theory or hypothesis driven. Based on theories and/or previous empirical research, CFA specifies a priori key aspects of a factor model including the number of factors and the relationships between the indicators and the underlying latent factors that they are hypothesized to measure. For example, in all CFAs conducted in this research each indicator was hypothesized to be influenced only by one latent factor, namely the construct it was hypothesized to measure. In a CFA model, the links between the indicators and an underlying factor are represented by factor loadings, which are the regression coefficients for predicting the indicators from their correspondent factor. Residuals (i.e., measurement errors) are also freely estimated. These residuals incorporate the variability in the given indicator that remains unaccounted for by the underlying factor or is caused by measurement error (Raykov \& Marcoulides, 2006).

Model identification determines whether it is possible to obtain a unique solution for all the freely estimated parameters in the hypothesized model (Brown, 2006; Wang \& Wang, 2012). For a model to be identified, measurement scales must be defined for latent factors in the model by either selecting or specifying marker indicators (e.g., by fixing the given factor loading) or by fixing the factor variance (Brown, 2006). To ensure identification, the number of known information in the sample variance-covariance matrix (i.e., indicators' variances and covariances) must not be less than the number of freely estimated parameters in the model (i.e., factor loadings, residual variances, inter-factor correlations; Brown, 2006).

Model estimation calculates model parameters by means of a fitting function that minimizes the discrepancy between the sample variance-covariance matrix and the model estimated matrix (Wang \& Wang, 2012). The most commonly used estimation method in CFA is maximum likelihood (ML) estimation (Brown, 2006; Byrne, 2011). "The underlying principles of ML estimation in CFA is to find the model parameters estimates that maximize the probability of observing the available data if the data were collected from the population again" (Brown, 2006, p. 73). Importantly, assumptions of ML are that the indicators are continuous variables and multivariate normal in distribution. Full information maximum 
likelihood (FIML; Arbuckle, 1996) is a robust estimator that is appropriate to use in case of small and medium violations of multivariate normality (Fan \& Wang, 1998). FIML uses all available information in the sample data (i.e., it does not purge cases having incomplete information), and is more efficient and less biased in dealing with missing data than the traditional approaches (e.g., listwise deletion, pairwise deletion) (Arbuckle, 1996; Wang \& Wang, 2012). FIML, as implemented in the software employed to analyze the data (i.e., Mplus; Muthén \& Muthén, 2017), was used in this research.

After obtaining model parameter estimates, researchers need to evaluate how well the model fits the data. To this end, they examine the overall goodness of fit, local areas of misfit (e.g., modification indices) and parameter estimates (Brown, 2006; Byrne, 2011; Geiser, 2013). The most commonly used goodness of fit indices include the $\chi^{2}$ statistic (Jöreskog, 1969) , the comparative fit index (CFI; Bentler, 1990), the Tucker-Lewis index (TLI; Tucker \& Lewis, 1973), the root-mean-square-error-of approximation (RMSEA; Steiger, 1990), and the standardized root mean square residual (SRMR; Bentler, 1995). Insignificant $\chi^{2}$ values, values of 0.950 and larger, for CFI and TLI, and 0.050 and smaller, for RMSEA and SRMR, are indicative of well-fitting models (Brown, 2006; Byrne, 2011; Geiser, 2013). Notably, for large samples (such as the sample I collected in this research), significant $\chi^{2}$ values are to be expected and do not suggest that the model does not fit well the data (for more extensive discussions of this aspect see Hodis \& Hodis, 2013 and references therein). An important strategy for checking local areas of model misfit is to examine the modification indices (MIs; Sörbom, 1989). A high MI value shows that the corresponding fixed parameter in the model should be freely estimated to improve model fit. However, it should be noted that any posthoc model modification must be justified on a substantive basis (Brown, 2006).

Finally, parameter estimates (e.g., their interpretability, magnitudes, and statistical significance) should be assessed. For instance, it is necessary to check whether there are improper parameter estimates (e.g., negative factor variances, correlations lower than -1 or higher than 1). The strengths of parameter estimates must be consistent with the substantive literature. For example, the magnitudes of the standardized factor loadings should be examined to evaluate whether the indicators provide appropriate measurements of the factor they are hypothesized to measure. Methodological research suggests that observed variables that have standardized factor loadings of 0.300 and higher are adequate/strong indicators of their underlying factors (Brown, 2006; Hodis, 2015, 2017; Hodis et al., 2017; Hodis \& Hodis, 2017).

LPA. LPA is a finite mixture modeling technique that identifies "a set of discrete, 
exhaustive, and non-overlapping latent classes of individuals based on individual responses to a set of indicators" (Tein, Coxe, \& Cham, 2013, p. 641) measuring the constructs of interest. LPA has been used successfully in prior research to identify groups of individuals with similar motivation profiles defined by promotion, prevention, assessment, and locomotion (Harring \& Hodis, 2016; Hodis et al., 2017). In these previous studies, LPA helped shed light on the complex interrelationships characterizing the interplay among promotion, prevention, assessment, and locomotion. In particular, Harring and Hodis (2016) and Hodis et al. (2017) employed LPA to identify subgroups of individuals who have similar patterns of endorsing promotion, prevention, assessment and locomotion. Therefore, when motivation profiles defined by promotion, prevention, assessment, and locomotion are studied, the grouping of students into distinct latent classes (i.e., groups that cannot be identified before conducting the analyses) is informed by their scores on items measuring each of the four motivation orientations. As an example, students classified (grouped) in a latent class may be characterized by having high levels of promotion and locomotion, low levels of prevention, and average levels of assessment.

LPA starts with obtaining profile indicators for constructs of interest (e.g., promotion, prevention, assessment, and locomotion) respectively (Harring \& Hodis, 2016; Hodis et al., 2017). In previous research (e.g., Harring \& Hodis, 2016; Hodis et al., 2017), these indicators were obtained by averaging corresponding item scores on promotion, prevention, assessment, and locomotion, respectively. In turn, these items are provided by validated scales such as Regulatory Focus Questionnaire (Higgins et al., 2001; for promotion and prevention) and Regulatory Mode Questionnaire (Kruglanski et al., 2000; for assessment and locomotion). In the next step, these four profile indicators are used to identify the motivation profiles that map the complex interrelationships among the four constructs (Harring \& Hodis, 2016; Hodis et al., 2017).

The most important and challenging step in LPA is latent class enumeration, that is, determining the number of latent classes that describes parsimoniously and effectively the heterogeneity of these interrelationships (Harring \& Hodis, 2016; Hodis et al., 2017). To do so, a combination of statistical indices, theoretical expectations, and substantive considerations are used to compare models with different numbers of classes (Nylund, Asparouhov, \& Muthén, 2007; Pastor \& Gagné, 2013; Peugh \& Fan, 2013; Tein et al., 2013).

The most commonly used statistical indices in latent class enumeration are information criteria, likelihood ratio statistical tests (LRTs), and classification-based approaches (e.g., entropy). Information criteria such as Akaike's Information Criterion (AIC; Akaike, 1973), 
Bayesian Information Criterion (BIC; Schwarz, 1978), and sample-size adjusted BIC (SABIC; Sclove, 1987) are descriptive indices for model comparisons that assist in selecting the most parsimonious and well-fitting model. For these indices, lower values indicate a better model fit.

Statistical tests that are often used to help inform the enumeration step in LPA include Lo-Mendell-Rubin likelihood ratio test (LMR; Lo, Mendall, \& Rubin, 2001) and bootstrap likelihood ratio test (BLRT; McLachlan \& Peel, 2000). Both of these tests evaluate the degree to which the specified model has a better fit than a model with one less class. For instance, if, in a BLRT, the $p$-value associated with the 5-class model is below the significance level (i.e., 0.05), this test suggests that the 5-class model fits better than the 4-class model.

Entropy is an index measuring classification accuracy. Larger values of entropy indicate a clearer separation of the classes (i.e., classes are significantly different from one another) and, thus, a better model fit. Jung and Wickrama (2008) suggested that entropy values above .70 show acceptable classification accuracy. Besides the fit indices, the sample sizes of the latent classes identified should also be taken into account (Ning \& Downing, 2015; Pastor \& Gagné, 2013). Specifically, because parameter estimates could be unstable in small classes (Pastor \& Gagné, 2013), recent research argued that any model including one or more classes having small sample sizes (i.e., smaller than 15) is unacceptable (Harring \& Hodis, 2016; Hodis et al., 2017).

Although statistical information plays an important role in latent class enumeration, the decision regarding the number of classes that account for the complex interrelationships among indicators also takes theoretical and substantive research into account. Specifically, plausible competing models are examined with regard to theoretical relevance and consistence with relevant substantive findings (Pastor \& Gagné, 2013). In addition, the graphic representations of the profiles identified by each of the competing models and their descriptive statistics should be checked for similarities/differences of latent classes within and across these models (Harring \& Hodis, 2016; Hodis et al., 2017; Pastor \& Gagné, 2013). In this way, researchers ensure that additional classes that are identified represent new patterns of interrelationships that are not well described by previously extracted classes. For example, in Study 1 of Hodis et al. (2017), an examination of the graphic representations of the profiles and descriptive statistics for each latent class in the two competing models (i.e., the four- and six-class models) showed that the model with four classes collated profiles that appeared as qualitatively distinct (i.e., having different patterns of absolute and relative strengths of the four indicators) in the 6-class model. Therefore, enumerating six classes 
offered an optimal representation of the data.

Once an appropriate solution has been selected, the last step in the analysis is to describe the identified latent classes (Harring \& Hodis, 2016; Hodis et al., 2017). To do so, the patterns of endorsing profile indicators (i.e., promotion, prevention, assessment, and locomotion) are compared and contrasted both within and across classes by means of analyzing the absolute and relative magnitudes of each orientation. The significance of the identified latent classes is highlighted by (a) examining differences across classes in criteria of interest and (b) mapping the motivation effects that are likely to be associated with each motivation profile (e.g., by discussing key characteristics of these profiles as indicated by existing theoretical and substantive research; Hodis et al., 2017).

In the last step of the quantitative phase, the motivation profiles identified were compared with regard to mean levels of grit, theory of intelligence, critical thinking, effort regulation, and perceptions/evaluations of academic success. For each criterion, tests of significance for the equality of means across these profiles were conducted.

\section{Phase Two: Qualitative Data Collection and Analysis}

This section presents the second phase of data collection and analysis which was done through a multiple-case study approach. The participants, data collection, and data analysis are discussed in the following.

Multiple-case study approach. The qualitative phase of this research used a multiplecase study approach to enable the in-depth investigation of a bounded entity such as a group within a real-life situation (Merriam, 1998; Yin, 2009). Yin (2009) recommended a multiplecase study as a suitable approach to answer 'how' and 'why' research questions. In this research, the case study was used to examine how the approaches to learning or learningrelated tasks/activities reported by the interview participants aligned with the nature of their respective motivation profiles (as identified in the quantitative phase). A group of participants having a common motivation profile was seen as a case.

A multiple-case study involves collecting and analyzing data from more than one case (Stake, 1995; Yin, 2009). Since five motivation profiles with distinct characteristics were identified in the quantitative phase, a multiple-case study was considered a suitable approach in this study. Stake (2006) viewed a multiple-case study as appropriate to investigate how a phenomenon performs in different contexts; each case has its own stories and contributes to a full understanding of the phenomenon. In this research, the phenomenon under investigation 
was how the characteristics of a given motivation profile identified in the quantitative phase, which was a snapshot of the interactions among the motivation orientations at the moment when the questionnaires were completed, were reflected in participants' approaches to learning or learning-related tasks/activities. Importantly, each of the five motivation profiles could be understood as a representation of a particular psychological context, which shaped the outcomes a student (who exhibited the given profile) tried to attain, the kinds of strategies and tactics she/he used to achieve the outcomes, and the way she/he tried to make sense of her/his experiences. The multiple-case study approach enabled me to understand how different individuals enacted the psychological context of their motivation profile with regard to university studies and related settings/topics. A variety of cases could provide more compelling evidence, which added to the robustness of the overall study (Yin, 2009). Moreover, the cross-case analysis allowed me to identify the similarities and differences among the motivation profiles.

Participants. Purposeful sampling was used to select eligible participants for the qualitative phase. The criteria for the participant recruitment were that they completed the questionnaires and expressed an interest in being contacted for the second phase of this research. After analyzing the quantitative data and identifying the motivation profiles in the first phase, I selected potential participants at random from all students who were classified as having a given motivation profile. I planned to recruit three to five participants per profile. However, when I contacted the potential participants, the majority of them did not respond to my invitations. In addition, some were no longer willing to take part in the research. Luckily, the students who agreed to participate recommended to me their classmates who participated in the questionnaire and expressed an interest in taking part in the interviews. I contacted them via Wechat and recruited 21 participants who met the criteria. However, two students suddenly withdrew before their interview times. Finally, a total of 19 students participated in the qualitative phase. Participants were provided with information sheets as well as consent forms to sign (See Appendices D and E). Table 5 presents the demographic information of the participants. In addition, for each participant, Table 5 records the probability with which the participant was classified in the corresponding latent class. The closer a probability to 1.0 , the stronger the confidence that a given participant's response profile can be classified unambiguously in the corresponding latent class.

I was aware that this method of recruiting interview participants had potential bias. Specifically, all participants were volunteers who were willing to talk about their learning experiences, which could suggest that they might be more motivated students. In addition, 
only three students reported academic achievement as being below 60 out of 100, which would suggest that the more academically successful students were more likely to participate in the qualitative phase. Moreover, more females volunteered for the qualitative interviews than males. Therefore, it was likely that these participants did not fully represent the sample in the quantitative phase.

Table 5

Demographic Information of Interview Participants

\begin{tabular}{|c|c|c|c|c|c|}
\hline $\begin{array}{l}\text { Motivation } \\
\text { profile }\end{array}$ & Pseudonyms & Gender & $\begin{array}{c}\text { Years at } \\
\text { university }\end{array}$ & Major & $\begin{array}{l}\text { Probability for } \\
\text { being } \\
\text { assigned to the } \\
\text { class }\end{array}$ \\
\hline \multirow[t]{4}{*}{ Class 1} & Lemon & Female & 2 & $\begin{array}{l}\text { Teaching Chinese to Speakers } \\
\text { of Other Languages }\end{array}$ & 0.979 \\
\hline & Lily & Female & 3 & International Business & 0.890 \\
\hline & Southerly & Female & 3 & International Business & 0.933 \\
\hline & Wang & Male & 3 & Labour and Social Security & 0.957 \\
\hline \multirow[t]{4}{*}{ Class 2} & Jasmine & Female & 2 & $\begin{array}{l}\text { Teaching Chinese to Speakers } \\
\text { of Other Languages }\end{array}$ & 0.904 \\
\hline & Linnaeus & Female & 2 & $\begin{array}{l}\text { Teaching Chinese to Speakers } \\
\text { of Other Languages }\end{array}$ & 0.958 \\
\hline & Qian & Female & 3 & Labour and Social Security & 0.820 \\
\hline & Sharon & Female & 2 & $\begin{array}{l}\text { Teaching Chinese to Speakers } \\
\text { of Other Languages }\end{array}$ & 0.940 \\
\hline \multirow[t]{3}{*}{ Class 3} & Quan & Male & 3 & Labour and Social Security & 0.997 \\
\hline & Vivian & Female & 3 & Labour and Social Security & 0.670 \\
\hline & $\mathrm{Zi}$ & Female & 2 & $\begin{array}{l}\text { Teaching Chinese to Speakers } \\
\text { of Other Languages }\end{array}$ & 0.934 \\
\hline \multirow[t]{3}{*}{ Class 4} & Kiva & Female & 1 & $\begin{array}{l}\text { Accounting (Sino-New } \\
\text { Zealand) }\end{array}$ & 0.510 \\
\hline & Mona & Female & 2 & $\begin{array}{l}\text { Teaching Chinese to Speakers } \\
\text { of Other Languages }\end{array}$ & 0.888 \\
\hline & Tian & Female & 3 & International Business & 1.000 \\
\hline \multirow[t]{5}{*}{ Class 5} & Della & Female & 3 & Labour and Social Security & 0.789 \\
\hline & Ivy & Female & 1 & $\begin{array}{l}\text { Accounting (Sino-New } \\
\text { Zealand) }\end{array}$ & 0.954 \\
\hline & Jacky & Male & 3 & International Business & 0.876 \\
\hline & Jenny & Female & 1 & $\begin{array}{l}\text { Teaching Chinese to Speakers } \\
\text { of Other Languages }\end{array}$ & 0.841 \\
\hline & $\mathrm{Wu}$ & Female & 2 & $\begin{array}{l}\text { Teaching Chinese to Speakers } \\
\text { of Other Languages }\end{array}$ & 0.625 \\
\hline
\end{tabular}

Qualitative instrument. Semi-structured individual interviews were used to collect 
qualitative data in the second phase. The main purpose of interviewing was to obtain an indepth understanding of the participant's experiences and perceptions as expressed in their words (Seidman, 2013; Taylor, Bogdan, \& Devault, 2015). In this research, interviews were conducted to shed light on the extent to which students' approaches to learning and learningrelated tasks/activities were consistent with the nature of their motivation profiles. The sample interview questions are contained in Appendix F.

The semi-structured interviews used in this research enabled me to predetermine the topics and the questions that directed the interviews, but also gave me flexibility regarding the wording or sequence of the questions during the interviewing process. This interview method could increase the comprehensiveness of data and ensure that the data collection was systematic for each participant (Johnson \& Christensen, 2017).

Data collection. In this section, I justify the online interviewing mode used in this research and describe the interviewing procedure. Issues of language choice, transcribing, translating, and relationships between the participants and me as the interviewer are also discussed.

Online interviewing. The individual interviews were conducted between January and February 2018. The interviews were conducted via Zoom, an online communication system, to allow me to have access to interviewees at a distance using both audio and visual means (Mann, 2016).

Interviewing process. The interview schedule was determined by the participants. Because they had to prepare for the final exams as the end of the semester approached, all video interviews with Zoom were conducted at times convenient to them. The participants chose to have the interviews at their dormitories or homes which made them feel comfortable. No other people were present during the interview. The participants were emailed the interview questions beforehand and given an opportunity to ask for further information. Each interview lasted 50 to 80 minutes. I began each interview with a brief introduction about the research and reassured them with regard to their confidentiality. During the interviews, I reminded them that there were no right or wrong answers to my questions, for all their stories and perceptions were meaningful to my research. I also used probes such as "Tell me a bit more please" and "Could you give me a specific example?" to encourage them to talk more. At the end of each interview, I asked the participants whether they had any additional comments or experiences to share with me. I also extended my appreciation for their participation and promised to email them the transcripts as soon as possible. As a consequence of building rapport with them, the participants appeared to be 
relaxed, confident, and open to me in the interviews. Participants in the qualitative phase received gifts (i.e., USB flash drives) after interviews were completed.

Language choice. The individual interviews were conducted in Chinese Mandarin to assist understandings and build relationships between the researcher and the participants (Cortazzi, Pilcher, \& Jin, 2011; Creswell, 2008). The language chosen for interviewing may affect the quality of the qualitative data, such as what and how much the participants might say (Cortazzi et al., 2011). I shared the same first language, Chinese Mandarin, as my participants. Considering that the participants' oral English proficiency was limited, I chose to interview them in their mother tongue, which enabled them to express their ideas fluently and freely.

Recording of data. All interviews were audio-recorded by Zoom. I also took field notes while interviewing to write down key responses and issues during the interviews. In order to capture the most accurate representation of the participants' ideas, I transcribed all the interviews in Chinese. All transcripts were emailed to the participants to check accuracy before I translated them into English. This method of member checking helped to ensure the trustworthiness of qualitative data (Creswell, 2008; Johnson \& Christensen, 2014; Mertens, 2006). All participants made general comments on their transcripts, such as comments about language and how they felt about the interviews. They also offered to keep in contact for further information. Three participants added their further thoughts to some questions in the interviews. All this suggested that they trusted me and were willing to share their experiences with me.

Translating the interview data from Chinese to English. Temple and Yong (2004) stated that researchers can act as the translators of their qualitative data if they see themselves as being neutral and objective in transmitting information. I taught English at a Chinese university for 10 years, and I am bilingual in English and Chinese, so I was able to translate all the interview transcripts by myself. Moreover, as both transcriber and translator, I could pay particular attention to the cultural meanings and interpretations in the process of translation and thus, gain a deeper understanding of the data (Cormier, 2018; Temple \& Yong, 2004).

I primarily aimed for 'conceptual equivalence' to translate the essential meanings conveyed by the participants' words rather than the exact word equivalence (Cormier, 2018). For example, “别人家的孩子' is a popular Chinese expression, which refers to the kind of role-model kids with whom parents compare their own children. They want their children to 
become those role models to show off and improve the status of the family. I translated this expression to "trophy kids", which conveys the similar meaning in the western culture. I translated in such a way to convey the essence of the original data and help the readers make sense of the participants' experiences.

However, by translating, the researchers might interpret the participants' words with their own ideologies, which could result in a less trustworthy representation of the participants' statements (Cormier, 2018). To ensure that the participants' ideas were appropriately communicated, I emailed both the transcripts and the translated versions to the participants to check the accuracy. As mentioned previously, the participants were bilingual, learning English as part of their degree programmes, so they were able to understand written English. I asked them to make comments or add thoughts. This method of member checking helped to ensure the accuracy of qualitative data (Cormier, 2018; Creswell, 2008).

Relationships between researcher and participants. The formation and development of relationships between me and the participants were influenced by several factors such as age, perceived status, and personal relationships. There was an asymmetry between my position and that of the participants. I was older and had worked as a lecturer in the target university. Although I had not taught the participants, they all called me 'Lao Shi' (i.e., teacher). This form of address was consistent with the Chinese culture, where people tend to show respect to people who are older and their teachers. My perceived status as a teacher and researcher might affect how these participants answered my questions. However, at the same time, I was able to position myself as an insider who was familiar with the teaching and learning contexts in the target university. In addition, my connection with the university made it easy for me to build rapport with these participants.

Moreover, during the research process, I developed warm relationships with the participants. After agreeing to participate in the interviews, all participants added me as friend on their Wechat accounts, giving me access to their Wechat Moments posting photos and videos of their daily life. They had the right to unfriend me at any time, or block me from viewing their Wechat Moments. However, we stayed virtual friends and followed each other's life experiences over time. Therefore, we knew each other well before the interviews and built a more balanced relationship; thus, they trusted me and were open to me during the interviews.

Data analysis. In this section, I describe and justify the coding process. I also give examples of how it impacted my analysis of data.

All qualitative data from individual interviews were coded and analysed with the 
assistance of NVivo 11. The coding was primarily an inductive and open process, although it was informed by the research questions, the quantitative results, and related literature. I followed the guidelines of thematic analysis by Braun and Clarke (2006). First, for each of the five cases, I read the interview transcripts a number of times to get familiar with the data that might be related to the research question (Braun \& Clarke, 2006). Then I translated these transcripts into English. The translation process enabled me to have a deeper understanding of the data. From each case, I selected one translated transcript that consisted of the richest or most interesting data. I analyzed these transcripts and generated initial codes. After that, I validated these codes across the remaining interviews for each case (Braun \& Clarke, 2006). Each code contained multiple extracts from different participants. I was also open to new codes that emerged from the remaining interviews. When new codes emerged, previously coded data were reviewed to see if those codes had been missed in the first coding. Therefore, the coding was an iterative process; the codes were reviewed and modified constantly. When coding was completed, I analyzed and identified the relationships between codes. This enabled me to categorize the codes into potential themes (Braun \& Clarke, 2006). Finally, I reviewed the themes to see whether they adequately reflected the qualitative data as a whole (Braun \& Clarke, 2006).

I coded the interview data using words or short phrases that "symbolically assigns a summative, salient, essence-capturing, and/or evocative attribute" for the data (Saldaña, 2013, p. 4). Several approaches of giving code names were used. I used the in vivo coding, selecting words or phrases taken from the language used by the participants as code names (Saldaña, 2013). Examples of in vivo codes are "I am not good at it", "It's useless to me" and "She echoed the textbook". The use of these code names enabled me to "prioritize and honor the participant's voice" (Saldaña, 2013, p. 91). In addition to in vivo coding, I used gerunds (verb + ing), such as "Working to achieve aspirations" and "Doing things step by step", as code names in order to highlight the actions in the data (Saldaña, 2013).

Memos were written to record my reflections on the coding process and my choices of code names, which allowed me to maintain a close connection between the data and the codes and have a deeper understanding of the phenomenon under investigation (Saldaña, 2013). The following example of my memos shows how I tried to define the codes and decide on my specific choices of code names.

This participant was involved in counterfactual thinking, reflecting on what would have happened if only she had made different decisions of applying for majors. The original code name, "counterfactual thinking” seems to be 
a theoretical term. "Reflecting on previous personal experiences" may be a better code name for being specific and descriptive. (Memo, 19 March, 2018)

Coding was "a cyclical act" (Saldaña, 2013, p. 8), which involved several cycles. In the first cycle, all codes were presented as free nodes in NVivo. There were no explicit connections among these codes. In the next cycle of coding, I attempted to develop links and group codes into categories or themes, using phrases which captured the essence of a set of repeating ideas reported by all participants having a common profile (Saldaña, 2013). Hierarchies of codes were then generated by using the function of parent and children nodes in Nvivo. For instance, for interview data from Class 2, the category of "Being cautious and careful" emerged by grouping the codes of "Following the course requirement", "Reviewing the key points before the final exams", "Checking the assignments carefully", and "Being cautious in making decisions". In addition, the research question (i.e., How do approaches to study reported by students align with their motivation profiles?) and the theoretical frameworks that guided this research (i.e., the regulatory focus theory and the regulatory mode theory) were used to inform the identification of important themes. In particular, guided by the research question and the theoretical frameworks, the themes such as "Alignment with strong assessment”, “Alignment with strong locomotion”, and "Alignment with dual strong promotion and prevention" began to emerge.

\section{Validity and Trustworthiness of the Research}

Adopting a mixed-methods research design, this research needed to ensure validity and trustworthiness of both quantitative and qualitative components as well as the sequential mixed-method design (Creswell \& Plano Clark, 2011; Johnson \& Christensen, 2014). This section first discusses issues regarding reliability, validity, and objectivity of the quantitative component and the parallel issues in the qualitative phase. Strategies to promote validity and trustworthiness of the mixed-method design are also addressed.

Quantitative phase. Mertens (2006) suggests that to evaluate the quality of quantitative research, researchers take into consideration three factors, that is, validity, reliability, and objectivity.

Validity and reliability. For quantitative research, validity refers to "the correctness or truthfulness of the inferences that are made from the results of the study" (Johnson \& Christensen, 2014, p. 279). Reliability refers to the extent to which the administration of an 
instrument that measures a construct produces consistent results (Mertens, 2006). As was discussed in the section of quantitative instruments, this research adapted existing instruments that have been shown to measure motivation orientations and other key motivation constructs in reliable and valid ways across samples and cultures. The questionnaires were also piloted with a group of Chinese students who helped identify potential threats such as confusing or repetitive items, thereby enhancing the reliability and validity of the instrument (Johnson \& Christensen, 2014). In addition, Confirmatory Factor Analyses (CFAs) were conducted in this research to provide evidence for construct validity (Mertens, 2006). Results of the CFA analyses are presented in the chapter of quantitative findings.

Objectivity. Mertens (2006) described objectivity as "how much the measurement instrument is open to influence by the beliefs and biases of the individuals who administer, score, or interpret it" (p. 388). In this research, I administered the questionnaires by myself, which ensured adopting a standard procedure of administration. Moreover, I was the sole researcher who was in charge of managing and analyzing the quantitative data. After the data collection was completed, I stored and managed the quantitative data using SPSS.

Qualitative phase. To ensure the trustworthiness of the qualitative phase, credibility, dependability, and conformability of findings should be considered (Mertens, 2006). Strategies that ensured these factors were summarized in Table 6. 
Table 6

Trustworthiness Strategies

\begin{tabular}{|c|c|c|}
\hline Trustworthiness & Strategies & Descriptions \\
\hline \multirow[t]{2}{*}{ Credibility } & $\begin{array}{l}\text { Member- } \\
\text { checking }\end{array}$ & $\begin{array}{l}\text { The transcripts and the translated versions were given to } \\
\text { participants to ensure the accuracy of the qualitative data. I } \\
\text { also asked further questions about things which were less } \\
\text { clear to me and they gave me richer explanations (Creswell, } \\
\text { 2008; Johnson \& Christensen, 2014; Mertens, 2006). }\end{array}$ \\
\hline & $\begin{array}{l}\text { Peer } \\
\text { reviewing }\end{array}$ & $\begin{array}{l}\text { My supervisors oversaw my research procedures and coding } \\
\text { process, which strengthened the credibility of my findings } \\
\text { (Johnson \& Christensen, 2014; Mertens, 2006). }\end{array}$ \\
\hline Dependability & $\begin{array}{l}\text { Articulation } \\
\text { of methods } \\
\text { and } \\
\text { procedures }\end{array}$ & $\begin{array}{l}\text { I described in great detail key aspects of the research, such } \\
\text { as the research design and processes of collecting and } \\
\text { analyzing data, which enables other researchers to judge the } \\
\text { dependability of the findings. }\end{array}$ \\
\hline \multirow[t]{3}{*}{ Conformability } & Reflexivity & $\begin{array}{l}\text { I kept research journals to explore my biases that might } \\
\text { affect the research process and conclusions. I often } \\
\text { questioned myself about whether my findings reflected the } \\
\text { data or only corresponded to some of my own assumptions } \\
\text { or predictions (Johnson \& Christensen, 2014). }\end{array}$ \\
\hline & $\begin{array}{l}\text { Use of direct } \\
\text { quotes and } \\
\text { careful } \\
\text { paraphrase }\end{array}$ & $\begin{array}{l}\text { I used direct quotes and careful paraphrases to avoid } \\
\text { introducing biased inferences (Johnson \& Christensen, } \\
\text { 2017). }\end{array}$ \\
\hline & $\begin{array}{l}\text { Peer } \\
\text { reviewing }\end{array}$ & $\begin{array}{l}\text { I discussed with my supervisors the translated transcripts } \\
\text { and coding }\end{array}$ \\
\hline
\end{tabular}

The mixed-methods design. Creswell and Plano Clark (2011) defined validity in mixed methods research as "employing strategies that address potential issues in data collection, data analysis, and the interpretation that might comprise the merging or connecting of the quantitative and qualitative strands of the study and the conclusions drawn from the combination" (p. 239). Strategies suggested by Creswell and Plano Clark (2011), and Johnson and Christensen (2017) were used to ensure the validity and trustworthiness of the mixed-methods design. These are summarized in Table 7. 
Table 7

Validity Strategies

\begin{tabular}{ll}
\hline Strategies & Descriptions \\
\hline $\begin{array}{l}\text { Participant } \\
\text { selection }\end{array}$ & $\begin{array}{l}\text { Participants in the quantitative and qualitative phases were recruited from } \\
\text { the same sample. Particularly, the participants in the qualitative phase } \\
\text { were purposefully selected according to the quantitative results (i.e., from } \\
\text { each motivation profile) among individuals who participated in the } \\
\text { quantitative phase (Creswell \& Plano Clark, 2011). }\end{array}$ \\
$\begin{array}{l}\text { Relative sample } \\
\text { size }\end{array}$ & $\begin{array}{l}\text { This research used a large sample size for the quantitative phase and a } \\
\text { small sample size for the qualitative phase (Creswell \& Plano Clark, }\end{array}$ \\
2011). \\
$\begin{array}{l}\text { Weakness } \\
\text { minimization }\end{array}$ & $\begin{array}{l}\text { The combination of quantitative and qualitative methods can compensate } \\
\text { for the limitations of each of these types of strategies and make use of } \\
\text { their respective strengths (Johnson \& Christensen, 2014).The quantitative } \\
\text { method enabled me gather data from a large number of students and } \\
\text { generalize the results, whilst the qualitative method allowed me to seek } \\
\text { in-depth understanding of different strategic approaches adopted by } \\
\text { students having distinct motivation profiles. }\end{array}$ \\
& $\begin{array}{l}\text { I used multiple methods of data collection (i.e., questionnaires, } \\
\text { interviews) and multiple sources of data (i.e., numerical data and } \\
\text { discourse data) to triangulate the findings (Johnson \& Christensen, } \\
\text { 2014). }\end{array}$ \\
\hline Triangulation &
\end{tabular}

\section{Ethical Considerations}

Ethical considerations are important for educational research and they aim to protect participants' rights and privacy (Johnson \& Christensen, 2017). I sought and obtained ethical approval from the Human Ethics Committee of Victoria University of Wellington. The research strictly adhered to the ethical guidelines proposed by Johnson and Christensen (2017), including avoiding deception, obtaining informed consent, protecting participants' rights, and ensuring anonymity and confidentiality.

Participant recruitment for the quantitative and qualitative phases in this research has been described in this chapter. The school heads and relevant lecturers were contacted to gain their approval to collect data from their students. No deception was involved. Although I had previously worked as a lecturer in the target university, I was not in charge of teaching or assessing any of the participants. Informed consent was obtained from all participants. The information sheet for the questionnaire participants stated that submission of this questionnaire implied consent for participating in the research. In this case, the questionnaire part of this research was anonymous, except for those students who agreed to be contacted for 
a subsequent interview and provided on the questionnaire booklets their names and contact information. For these students, this research was confidential. They were provided with separate information sheets and consent forms written in both English and Chinese. Pseudonyms of participants in the qualitative phase were used to keep their identities confidential. The students had the freedom to decline to participate in this research or withdraw at any time. To preserve confidentiality, all collected data were only accessible to myself and my supervisors.

\section{Chapter Summary}

In this chapter I detailed the methodology of this research. A mixed methods sequential explanatory design was used in this study, in which the quantitative phase preceded the qualitative one. Each phase was presented in detail including the selection of the participants, the instruments, data collection and analysis. Finally, issues relating to validity and trustworthiness of this research as well as ethical considerations were addressed. This leads to the quantitative and qualitative findings which are reported in the next two chapters. 


\section{Chapter Four Quantitative Findings}

In this chapter, I report the quantitative findings for the first phase of this research. I present the results of CFA and LPA analyses as well as the similarities and differences among the motivation profiles in terms of the key outcomes investigated in the research.

\section{Results of CFA Analyses}

In this section, I report the results of the analyses investigating the quality of the measurement models for each of the constructs examined in this research. To this end, 1- and 2-factor confirmatory factor analyses (CFAs) were conducted in Mplus, version 8 (Muthén \& Muthén, 2017). I begin with the presentation of the results with the model examining promotion and prevention constructs, followed by those involving assessment and locomotion.

The skew of the promotion items ranged between -1.131 and 0.025 ; their kurtosis ranged between -0.662 and 0.933 . The skew of prevention items ranged between -1.219 and 0.253 . For these items, kurtosis ranged between -0.800 and 0.917 . The skew of locomotion items ranged between -1.682 and 0.269 . For these items, kurtosis ranged between -.610 and 3.194. The skew of the assessment items ranged between -0.562 and 0.372 ; their kurtosis ranged between -0.826 and -0.354 . Violations of the multivariate normality (MVN) are suspected only when absolute values of skew are larger than 2.00 and the values of kurtosis exceed 7.00 (Curran, West, \& Finch, 1996). Therefore, these results reveal that there were no problematic violations of the MVN for any of the items measuring promotion, prevention, assessment, and locomotion.

In this research items that had standardized factor loadings of or above 0.500 are regarded as strong indicators of their corresponding constructs. Widaman, Little, Preacher, and Sawalani (2011) suggested that a standardized loading of 0.500 corresponds to a mean inter-item correlation (MIC) of about 0.250. In turn, a MIC of 0.250 falls in the interval of $(0.150,0.500)$ where MIC values for scales should lie (Clark \& Watson, 1995). The selection of 0.500 as the benchmark for strong standardized factor loadings is consistent with previous studies on the Regulatory Focus Questionnaire (RFQ; Higgins et al., 2001) and Regulatory Mode Questionnaire (RMQ; Kruglanski et al., 2000) (Hodis, 2015; Hodis, 2017; Hodis \& Hodis, 2017). 


\section{CFA models for promotion.}

The 1-factor CFA model for promotion: 6 indicators. This model had a very good fit to the data: Chi-square $(9, N=866)=24.423, p=.004 ; \mathrm{CFI}=0.973 ; \mathrm{TLI}=0.955 ; \mathrm{RMSEA}=$ 0.044, with the $90 \%$ confidential interval (CI) for RMSEA being [0.023, 0.065]. Results in Table 8 show that only two items (i.e., PRO_2 and PRO_4) had standardized factor loadings below 0.500. However, the magnitude of the loading for PRO_4 (i.e., 0.465) was only slightly smaller than 0.500 , thus suggesting that PRO_4 is a robust indicator of promotion in this sample. PRO_2 (i.e., “Accomplishing things motivates me to work even harder”) had a very low factor loading (i.e., 0.269), which indicates that it is a weak indicator of promotion. Because I wanted to use strong indicators of the given construct for latent profile analyses (LPAs), I decided to omit PRO_2 for the next phase of this research.

Table 8

Maximum-Likelihood Estimates of Descriptive Statistics and 1-Factor CFA Standardized Loadings and Standard Errors of Promotion Items

\begin{tabular}{lcccccc}
\hline Item & $\begin{array}{c}\text { Standardized } \\
\text { factor loading }\end{array}$ & $\begin{array}{c}\text { Standard } \\
\text { error }\end{array}$ & Skew & Kurtosis & $\begin{array}{c}\text { Mean } \\
\text { item }\end{array}$ & $\begin{array}{c}\text { Variance } \\
\text { item }\end{array}$ \\
\hline PRO_1 & $0.512 / 0.526$ & $0.034 / 0.034$ & -0.175 & -0.424 & 4.369 & 1.856 \\
PRO_2 & 0.269 & 0.039 & -1.131 & 0.933 & 5.520 & 2.022 \\
PRO_3 & $0.572 / 0.569$ & $0.033 / 0.034$ & -0.186 & -0.217 & 4.502 & 1.507 \\
PRO_4 & $0.465 / 0.457$ & $0.035 / 0.036$ & -0.078 & -0.301 & 4.223 & 1.907 \\
PRO_5 & $0.529 / 0.538$ & $0.034 / 0.034$ & 0.025 & -0.253 & 4.275 & 1.877 \\
PRO_6 & $0.591 / 0.579$ & $0.032 / 0.033$ & -0.414 & -0.662 & 4.870 & 2.373 \\
\hline
\end{tabular}

Note. Item numbers, in the first column, correspond to those in the RFQ scale (Higgins et al., 2001). PRO = promotion. The italicized values correspond to the model with five promotion items.

The 1-factor CFA model for promotion: 5 indicators. This model had an excellent fit: Chi-square $(5, N=886)=14.130, p=.015 ; \mathrm{CFI}=0.983 ; \mathrm{TLI}=0.965 ; \mathrm{RMSEA}=0.045,90 \%$ CI for RMSEA being [0.018, 0.074]. The standardized parameter estimates associated with this model are summarized in italics in Table 8. These results indicate that all the five promotion indicators retained had strong relationships with the construct in this sample.

\section{CFA models for prevention.}

The 1-factor CFA model for prevention: 5 indicators. This model fitted the data well: Chi-square $(5, N=886)=23.487, p<.001 ; \mathrm{CFI}=0.988$; TLI $=0.975 ; \mathrm{RMSEA}=0.065$, with 90\% CI for RMSEA being [0.040, 0.092]. As presented in Table 9, four of the five indicators 
had standardized loadings larger than 0.500. The loading of PRE_5 (i.e., "Not being careful enough has gotten me into trouble at times") was very low (i.e., 0.105), thus suggesting it is a very weak indicator of prevention in this sample. Therefore, PRE_5 was not retained for the following analyses.

Table 9

Maximum-Likelihood Estimates of Descriptive Statistics and 1-Factor CFA Standardized Loadings and Standard Errors of Prevention Items

\begin{tabular}{ccccccc}
\hline Item & $\begin{array}{c}\text { Standardized } \\
\text { factor loading }\end{array}$ & $\begin{array}{c}\text { Standard } \\
\text { error }\end{array}$ & Skew & Kurtosis & $\begin{array}{c}\text { Mean } \\
\text { item }\end{array}$ & $\begin{array}{c}\text { Variance } \\
\text { item }\end{array}$ \\
\hline PRE_1 & $0.876 / 0.875$ & $0.014 / 0.014$ & -1.219 & 0.917 & 5.602 & 2.131 \\
PRE_2 & $0.718 / 0.717$ & $0.019 / 0.019$ & -1.131 & 0.667 & 5.590 & 2.082 \\
PRE_3 & $0.605 / 0.606$ & $0.024 / 0.024$ & -0.442 & -0.050 & 4.625 & 1.726 \\
PRE_4 & $0.820 / 0.822$ & $0.015 / 0.015$ & -1.013 & 0.533 & 5.501 & 2.015 \\
PRE_5 & 0.105 & 0.036 & 0.253 & -0.800 & 3.426 & 2.646 \\
\hline
\end{tabular}

Note. Item numbers, in the first column, correspond to those in the RFQ scale (Higgins et al., 2001). PRE = prevention. Items in italics (i.e., PRE_1, PRE_2, PRE_4, and PRE_5) were reverse scored before the analyses. The italicized values correspond to the model with four prevention items.

The 1-factor CFA model for prevention: 4 indicators. This model had a good fit: Chisquare $(2, N=886)=12.388, p=.002 ; \mathrm{CFI}=0.993$; $\mathrm{TLI}=0.979 ; \mathrm{RMSEA}=0.077$, with the 90\% CI for RMSEA being [0.040, 0.120]. Results reported in Table 9 reveal that all the four indicators retained had standardized loadings exceeding 0.500 , and thus, they are strong indicators of prevention. To sum up, taking the results of the above analyses into consideration, five promotion items and four prevention items were used when investigating the 2-factor CFA model involving these constructs. This analysis was undertaken to evaluate the extent to which promotion and prevention were related.

The 2-factor CFA model for promotion (5 indicators) and prevention (4 indicators). No cross-loadings or correlated residuals were included in the 2-factor CFA model for promotion (5 indicators) and prevention (4 indicators) under investigation. This model had an excellent fit to the data: Chi-square $(26, N=886)=65.155, p<.001 ; \mathrm{CFI}=0.981 ; \mathrm{TLI}=$ 0.973; RMSEA $=0.041$, with the $90 \%$ CI for RMSEA being [0.029, 0.054]. A summary of the standardized parameter estimates associated with this model, which is included in Table 10 , suggests that all of the factor loadings were statistically significant and of sizeable strength. Moreover, the promotion and prevention factors were found to have a very weak correlation and this association was not statistically significant (i.e., $r=0.058 ; p=.188$ ). These results reveal that no spurious relations among promotion indicators, prevention 
indicators, and their corresponding constructs were apparent. Taken together, the findings suggest that the five promotion indicators and the four prevention indicators measured appropriately these two constructs in this population.

Table 10

Maximum-Likelihood Estimates of 2-Factor CFA Standardized Loadings and Standard Errors of Promotion and Prevention Items

\begin{tabular}{lccc}
\hline Item & Scale & $\begin{array}{c}\text { Standardized factor } \\
\text { loading }\end{array}$ & Standard error \\
\hline PRO_1 & PRO & 0.526 & 0.034 \\
PRO_3 & PRO & 0.571 & 0.034 \\
PRO_4 & PRO & 0.456 & 0.036 \\
PRO_5 & PRO & 0.538 & 0.034 \\
PRO_6 & PRO & 0.578 & 0.033 \\
PRE_1 & PRE & 0.875 & 0.014 \\
PRE_2 & PRE & 0.718 & 0.019 \\
PRE_3 & PRE & 0.607 & 0.024 \\
PRE_4 & PRE & 0.821 & 0.015 \\
\hline
\end{tabular}

Note. Item numbers, in the first column, correspond to those in the RFQ Scale (Higgins et al., 2001). $\mathrm{PRO}=$ promotion; $\mathrm{PRE}=$ prevention. Three prevention items (i.e., PRE_1, PRE_2, and PRE_4) were reverse scored before the analysis.

\section{CFA models for locomotion.}

The 1-factor CFA model for locomotion: 12 indicators. This model had a poor fit to the data: Chi-square $(54, N=866)=278.742, p<.001 ; \mathrm{CFI}=0.892 ; \mathrm{TLI}=0.868 ; \mathrm{RMSEA}=$ 0.069 , with the $90 \%$ CI for RMSEA being [0.061, 0.077]. Results presented in Table 11 show that six items (i.e., LOC_1, LOC_2, LOC_3, LOC_6, LOC_7, LOC_8 and LOC_10) had standardized factor loadings smaller than 0.500. However, the magnitudes of the loadings for LOC_2, LOC_7, LOC_8 and LOC_10 (i.e., 0.496, 0.495, 0.491 and 0.477 , respectively) were only slightly less than 0.500 , which indicates that these three items are robust indicators of locomotion in this sample. LOC_1, LOC_3 and LOC_6 had low factor loadings (i.e., 0.313, 0.290 , and 0.418 , respectively), thus suggesting that these items are weaker indicators of locomotion. As I aimed to use strong indicators of all constructs for LPAs, I omitted these three items (i.e., "I don't mind doing things even if they involve extra effort"; "I feel excited just before I am about to reach a goal"; "When I finish one project, I immediately start a new one") for the next analyses. 
Table 11

Maximum-Likelihood Estimates of Descriptive Statistics and 1-Factor CFA Standardized Loadings and Standard Errors of Locomotion Items

\begin{tabular}{lcccccc}
\hline Item & $\begin{array}{c}\text { Standardized } \\
\text { factor loading }\end{array}$ & $\begin{array}{c}\text { Standard } \\
\text { error }\end{array}$ & Skew & Kurtosis & $\begin{array}{c}\text { Mean } \\
\text { item }\end{array}$ & $\begin{array}{c}\text { Variance } \\
\text { item }\end{array}$ \\
\hline LOC_1 & 0.313 & 0.034 & -0.796 & 0.206 & 5.164 & 2.112 \\
LOC_2 & $0.496 / 0.494$ & $0.029 / 0.029$ & 0.236 & -0.610 & 3.515 & 2.284 \\
LOC_3 & 0.290 & 0.034 & -1.682 & 3.194 & 6.020 & 1.456 \\
LOC_4 & $0.546 / 0.546$ & $0.027 / 0.028$ & -0.310 & -0.460 & 4.764 & 2.052 \\
LOC_5 & $0.654 / 0.663$ & $0.023 / 0.023$ & -0.272 & -0.263 & 4.613 & 1.905 \\
LOC_6 & 0.418 & 0.031 & 0.269 & -0.297 & 3.699 & 1.915 \\
LOC_7 & $0.495 / 0.494$ & $0.029 / 0.029$ & -0.548 & -0.441 & 5.009 & 2.429 \\
LOC_8 & $0.491 / 0.462$ & $0.029 / 0.030$ & 0.006 & -0.498 & 4.089 & 2.038 \\
LOC_9 & $0.522 / 0.518$ & $0.028 / 0.028$ & -0.277 & -0.502 & 4.577 & 2.176 \\
LOC_10 & $0.477 / 0.470$ & $0.030 / 0.030$ & -0.249 & -0.462 & 4.641 & 1.955 \\
LOC_11 & $0.517 / 0.518$ & $0.028 / 0.029$ & -0.237 & -0.599 & 4.590 & 1.946 \\
LOC_12 & $0.764 / 0.774$ & $0.019 / 0.019$ & -0.379 & -0.045 & 4.854 & 1.621 \\
\hline
\end{tabular}

Note. Item numbers, in the first column, correspond to those in the RMQ scale (i.e., Kruglanski et al., 2000). LOC = locomotion. The values in italics correspond to the model with nine locomotion items.

The 1-factor CFA model for locomotion: 9 indicators. This model fitted the data well: Chi-square $(27, N=886)=124.321, p<.001 ; \mathrm{CFI}=0.942 ; \mathrm{TLI}=0.923 ; \mathrm{RMSEA}=0.064$, 90\% CI for RMSEA being [0.053, 0.075]. The standardized parameter estimates associated with this model are reported in Table 11. Five items (i.e., LOC_4, LOC_5, LOC_9, LOCO_11 and LOC_12) had loadings larger than 0.500; the other four items (i.e., LOC_2, LOC_7, LOC_8, and LOC_10) had loadings that were only slightly smaller than 0.500 (i.e., $0.494,0.494,0.462$, and 0.470$)$. These results indicate that all the nine indicators of locomotion are generally robust measures of this construct in the sample.

\section{CFA models for assessment.}

The 1-factor CFA models for assessment: 12 indicators. The model with all the twelve indicators had a poor fit: Chi-square $(54, N=886)=436.390, p<.001 ; \mathrm{CFI}=0.837$; TLI $=$ 0.800; RMSEA $=0.089,90 \%$ CI for RMSEA being [0.082, 0.097]. Results in Table 12 indicate that the loadings of ASE_5 and ASE_9 were low (i.e., 0.436 and 0.350), thus suggesting these two items are relatively weak indicators of assessment. The other items had loadings which exceeded 0.500 or were slightly lower than 0.500. ASE_5 (i.e., "I spend a lot of time thinking about ways others could improve themselves") and ASE_9 (i.e., "I am very 
self-critical and self-conscious about what I am saying") were omitted for the following analyses.

Table 12

Maximum-Likelihood Estimates of Descriptive Statistics and 1-Factor CFA Standardized Loadings and Standard Errors of Assessment Items

\begin{tabular}{lcccccc}
\hline Item & $\begin{array}{c}\text { Standardized } \\
\text { factor loading }\end{array}$ & $\begin{array}{c}\text { Standard } \\
\text { error }\end{array}$ & Skew & Kurtosis & $\begin{array}{c}\text { Mean } \\
\text { item }\end{array}$ & $\begin{array}{c}\text { Variance } \\
\text { item }\end{array}$ \\
\hline ASE_1 & $0.541 / 0.605$ & $0.028 / 0.028$ & -0.438 & -0.354 & 4.778 & 2.031 \\
ASE_2 & $0.539 / 0.527$ & $0.028 / 0.030$ & 0.039 & -0.764 & 4.097 & 2.338 \\
ASE_3 & 0.536 & 0.028 & 0.372 & -0.716 & 3.354 & 2.441 \\
ASE_4 & $0.597 / 0.593$ & $0.026 / 0.028$ & -0.383 & -0.608 & 4.631 & 2.375 \\
ASE_5 & 0.436 & 0.031 & 0.082 & -0.766 & 3.593 & 2.393 \\
ASE_6 & $0.613 / 0.517$ & $0.025 / 0.031$ & -0.152 & -0.468 & 4.186 & 2.235 \\
ASE_7 & 0.467 & 0.030 & -0.111 & -0.711 & 4.188 & 2.449 \\
ASE_8 & 0.493 & 0.029 & 0.114 & -0.826 & 3.875 & 2.694 \\
ASE_9 & 0.350 & 0.033 & -0.249 & -0.710 & 4.565 & 2.341 \\
ASE_10 & $0.663 / 0.540$ & $0.023 / 0.030$ & 0.160 & -0.704 & 3.756 & 2.277 \\
ASE_11 & $0.517 / 0.599$ & $0.029 / 0.028$ & -0.407 & -0.443 & 4.765 & 2.071 \\
ASE_12 & $0.506 / 0.576$ & $0.029 / 0.029$ & -0.562 & -0.366 & 4.905 & 2.402 \\
\hline
\end{tabular}

Note. Item numbers, in the first column, correspond to those in the RMQ scale (i.e.,

Kruglanski et al., 2000). ASE = assessment. The values in italics correspond to the model with seven assessment items and correlated residuals for ASE_6 and ASE_10.

The 1-factor CFA models for assessment: 10 indicators. The model with 10 indicators of assessment had a poor fit: Chi-square $(35, N=886)=313.664, p<.001 ; \mathrm{CFI}=0.860$; TLI $=$ 0.820 ; RMSEA $=0.095,90 \%$ CI for RMSEA being [0.085, 0.105]. The results reveal that all the items except ASE_7 and ASE_8 had loadings larger than 0.500. The lower loadings of ASE_7 and ASE_8 (i.e., 0.479 and 0.490) suggest these two items are not as strong as the remaining eight assessment measures. Attempts to improve the model fit via post-hoc changes suggested by the modification indices (e.g., estimation of correlated residuals) were not successful.

Therefore, I decided not to retain these two items (i.e., "I often feel that I am being evaluated by others"; "I am a critical person").

The 1-factor CFA models for assessment: 8 indicators. The model with eight indicators still fitted the data poorly: Chi-square $(20, N=886)=209.656, p<.001 ; \mathrm{CFI}=0.878 ; \mathrm{TLI}=$ 0.829; RMSEA $=0.103,90 \%$ CI for RMSEA being [0.091, 0.116]. The results show that all the eight items had loadings larger than 0.500 . However, an examination of the modification indices suggests that a residual covariance (between ASE_3 and ASE_10) was not modeled. Therefore, I decided to estimate this parameter in the following CFA model. 
The model with eight indicators and a residual covariance between ASE_3 and ASE_10 had a less than optimal fit to the data: Chi-square $(19, N=886)=145.495$, $p<.001 ; \mathrm{CFI}=0.918 ; \mathrm{TLI}=0.880 ; \mathrm{RMSEA}=0.087,90 \% \mathrm{CI}$ for RMSEA being $[0.074,0.100]$. The results show that only ASE_3 had a loading smaller than 0.500 (i.e., 0.459), thus suggesting it is a weaker indicator of assessment. I decided to omit ASE_3 (i.e., "I often evaluate other people's plans") in the next model.

The 1-factor CFA models for assessment: 7 indicators. The model with seven indicators still had a suboptimal fit: Chi-square $(14, N=886)=100.820, p<.001 ; \mathrm{CFI}=0.930 ;$ TLI $=$ 0.895; RMSEA $=0.084,90 \%$ CI for RMSEA being $[0.069,0.099]$. The results indicate that all the seven indicators had loadings exceeding 0.500. An examination of the modification indices reveals that the largest modification index corresponded to a correlation between ASE_6 and ASE_10. Therefore, I decided to estimate this parameter in the subsequent CFA model.

The 1-factor model that estimated freely a residual correlation between ASE_6 and ASE_10 fitted the data well: Chi-square $(13, N=886)=60.004, p<.001 ; \mathrm{CFI}=0.962$; $\mathrm{TLI}=0.939 ; \mathrm{RMSEA}=0.064,90 \% \mathrm{CI}$ for RMSEA being [0.048, 0.081]. The results in Table 12 indicate that all the seven indicators had loadings above 0.500 , thus suggesting they are strong indicators of assessment. Therefore, I retained all the seven indicators (i.e., ASE_1, ASE_2, ASE_4, ASE_6, ASE_10, ASE_11, and ASE_12) for the following phase. Notably, this 1 -factor model for assessment had a good fit only after it estimated freely the residual correlation between ASE_6 and ASE_10. In sum, taking the results of the above mentioned analyses into consideration, I retained nine locomotion items, seven assessment items and I modelled a residual covariance for the following two-factor CFA analysis.

The 2-factor CFA model for locomotion (9 indicators) and assessment (7 indicators plus estimated residual covariance). This model had a nearly acceptable fit to the data: Chi-square $(102, N=886)=377.272, p<.001 ; \mathrm{CFI}=0.912 ; \mathrm{TLI}=0.897 ; \mathrm{RMSEA}=0.055$, with the $90 \%$ CI for RMSEA being [0.049, 0.061]. All of the factor loadings were larger than 0.500 . However, an examination of the modification indices suggests that an additional unmodeled residual covariance (between LOC_5 and LOC_7) affected negatively the model fit. Hence, I decided to estimate this parameter in the following CFA model. The subsequent 2-factor model had a good fit to the data: Chi-square $(101, N=886)=343.582, p<.001 ; \mathrm{CFI}=0.923$; TLI $=0.908$; RMSEA $=0.052$, with the $90 \%$ CI for RMSEA being [0.046, 0.058]. As presented in Table 13, all of the factor loadings were statistically significant and of sizeable strength. The locomotion and assessment factors were found to be positively correlated (i.e., $r=0.420$; 
$p<.001)$. Taken together, the findings suggest that the nine locomotion indicators and the seven assessment indicators had strong relationships with the two constructs they were hypothesized to measure in this population.

Table 13

Maximum-Likelihood Estimates of 2-Factor CFA Standardized Loadings and Standard Errors of Locomotion and Assessment Items

\begin{tabular}{llcc}
\hline Item & Scale & Standardized factor loading & Standard error \\
\hline LOC_2 & LOC & 0.498 & 0.029 \\
LOC_4 & LOC & 0.551 & 0.027 \\
LOC_5 & LOC & 0.634 & 0.025 \\
LOC_7 & LOC & 0.460 & 0.031 \\
LOC_8 & LOC & 0.480 & 0.030 \\
LOC_9 & LOC & 0.521 & 0.028 \\
LOC_10 & LOC & 0.489 & 0.030 \\
LOC_11 & LOC & 0.522 & 0.029 \\
LOC_12 & LOC & 0.769 & 0.020 \\
ASE_1 & ASE & 0.598 & 0.028 \\
ASE_2 & ASE & 0.531 & 0.030 \\
ASE_4 & ASE & 0.578 & 0.028 \\
ASE_6 & ASE & 0.515 & 0.031 \\
ASE_10 & ASE & 0.521 & 0.031 \\
ASE_11 & ASE & 0.621 & 0.027 \\
ASE_12 & ASE & 0.585 & 0.028 \\
\hline
\end{tabular}

Note. Item numbers, in the first column, correspond to those the RMQ scale (Kruglanski et al., 2000). LOC $=$ locomotion; ASE $=$ assessment. The final 2-factor CFA model freely estimated the residual covariance between ASE_6 and ASE_10 and the residual covariance between LOC_5 and LOC_7.

CFA models for grit. Grit has two facets: consistency of interest and perseverance of effort. For items measuring consistency of interest, the skew ranged between -0.066 and 0.498 ; their kurtosis ranged between -0.846 and -0.031 . For items gauging perseverance of effort, the skew ranged between -0.466 and 0.067 ; their kurtosis ranged between -0.699 and -0.355 . These results suggest that there were no problematic violations of the MVN for any of the grit items (Curran et al., 1996).

The 1-factor CFA model for consistency of interest: 4 indicators. This model had an excellent fit to the data: Chi-square $(2, N=866)=0.044, p=.978 ; \mathrm{CFI}=1.000 ;$ TLI $=1.000$; RMSEA $=0.000$, with the $90 \%$ CI for RMSEA being [0.000, 0.000]. Results in Table 14 reveal that CON_1 and CON_3 had standardized factor loadings larger than 0.500. CON_4 had a loading that was slightly lower than 0.500 (i.e., 0.472), and thus, it had a robust relationship with the construct under study. CON_2 had a low factor loading (i.e., 0.382), 
which indicates that it is a weaker indicator of this construct. Therefore, I decided to omit CON_2 (i.e., "New ideas and projects sometimes distract me from previous ones") for the subsequent analyses.

Table 14

Maximum-Likelihood Estimates of Descriptive Statistics and 1-Factor Standardized Loadings and Standard Errors of Consistency of Interest Items

\begin{tabular}{lcccccc}
\hline Item & $\begin{array}{c}\text { Standardized } \\
\text { factor loading }\end{array}$ & $\begin{array}{c}\text { Standard } \\
\text { error }\end{array}$ & Skew & Kurtosis & $\begin{array}{c}\text { Mean } \\
\text { item }\end{array}$ & $\begin{array}{c}\text { Variance } \\
\text { item }\end{array}$ \\
\hline CON_1 & 0.566 & 0.039 & -0.066 & -0.769 & 4.137 & 2.314 \\
CON_2 & 0.382 & 0.040 & 0.498 & -0.031 & 3.199 & 1.690 \\
CON_3 & 0.625 & 0.040 & 0.087 & -0.704 & 3.644 & 2.351 \\
CON_4 & 0.472 & 0.039 & 0.028 & -0.846 & 4.105 & 2.496 \\
\hline
\end{tabular}

Note. Item numbers, in the first column, correspond to those in the Grit-S Scale (Duckworth \& Quinn, 2009). CON = consistency of interest. All the items were reverse scored before the analysis.

The 1-factor CFA model for perseverance of effort: 4 indicators. This model had an acceptable fit to the data: Chi-square $(2, N=866)=16.714, p<.001 ; \mathrm{CFI}=0.976$; $\mathrm{TLI}=$ 0.929; RMSEA $=0.091$, with the 90\% CI for RMSEA being [0.054, 0.134]. Results in Table 15 reveal that PER_3 and PER_4 had standardized factor loadings larger than 0.500. PER_1 and PER_2 had low factor loadings (i.e., 0.351 and 0.281), which indicates that they are weaker indicators of this construct. This is why I decided to omit PER_1 (i.e., "I finish whatever I begin") and PER_2 (i.e., "Setbacks don't discourage me") for the subsequent analyses.

Table 15

Maximum-Likelihood Estimates of Descriptive Statistics and 1-Factor CFA Standardized Loadings and Standard Errors of Perseverance of Effort Items

\begin{tabular}{lcccccc}
\hline Item & $\begin{array}{c}\text { Standardized } \\
\text { factor loading }\end{array}$ & $\begin{array}{c}\text { Standard } \\
\text { error }\end{array}$ & Skew & Kurtosis & $\begin{array}{c}\text { Mean } \\
\text { item }\end{array}$ & $\begin{array}{c}\text { Variance } \\
\text { item }\end{array}$ \\
\hline PER_1 & 0.351 & 0.034 & -0.466 & -0.355 & 4.921 & 2.174 \\
PER_2 & 0.281 & 0.035 & -0.291 & -0.699 & 4.605 & 2.395 \\
PER_3 & 0.820 & 0.032 & -0.135 & -0.376 & 4.295 & 1.921 \\
PER_4 & 0.779 & 0.031 & -0.067 & -0.459 & 4.190 & 1.903 \\
\hline
\end{tabular}

Note. Item numbers, in the first column, correspond to those in the Grit-S scale (Duckworth \& Quinn, 2009). PER = perseverance of effort.

The 1-factor CFA models for grit: 8 indicators. The model with all eight indicators (i.e., four items for consistency of interest and four items for perseverance of effort) fitted the data poorly: Chi-square $(20, N=866)=244.596, p<.001 ; \mathrm{CFI}=0.792 ; \mathrm{TLI}=0.709$; 
RMSEA $=0.113$, with the $90 \%$ CI for RMSEA being [0.100, 0.125]. The summary of the standardized parameter estimates in Table 16 reveal that all the items except PER_3 and PER_4 had standardized factor loadings smaller than 0.500 .

The model measuring grit by means of the strongest indicators for consistency of interest ( 3 items) and perseverance of effort ( 2 items) also had a poor fit to the data: Chisquare $(5, N=866)=134.409, p<.001 ; \mathrm{CFI}=0.840 ; \mathrm{TLI}=0.681 ; \mathrm{RMSEA}=0.171$, with the $90 \%$ CI for RMSEA being [0.147, 0.196]. Results in italics in Table 16 indicate that all the items except PER_3 and PER_4 had standardized factor loadings below 0.500. These findings suggest that in this population a 1-factor conceptualization of grit, which collates both consistency of interest and perseverance of effort, is not supported by the data.

Table 16

Maximum-Likelihood Estimates of Descriptive Statistics and 1-Factor CFA Standardized Loadings and Standard Errors of Grit Items (i.e., Both Consistency of Interest and Perseverance of Effort Items)

\begin{tabular}{lcccccc}
\hline Item & $\begin{array}{c}\text { Standardized } \\
\text { factor loading }\end{array}$ & $\begin{array}{c}\text { Standard } \\
\text { error }\end{array}$ & Skew & Kurtosis & $\begin{array}{c}\text { Mean } \\
\text { item }\end{array}$ & $\begin{array}{c}\text { Variance } \\
\text { item }\end{array}$ \\
\hline CON_1 & $0.381 / 0.351$ & $0.035 / 0.035$ & -0.066 & -0.769 & 4.137 & 2.314 \\
CON_2 & 0.140 & 0.038 & 0.498 & -0.031 & 3.199 & 1.690 \\
CON_3 & $0.382 / 0.359$ & $0.035 / 0.035$ & 0.087 & -0.704 & 3.644 & 2.351 \\
CON_4 & $0.344 / 0.315$ & $0.035 / 0.035$ & 0.028 & -0.846 & 4.105 & 2.496 \\
PER_1 & 0.383 & 0.034 & -0.466 & -0.355 & 4.921 & 2.174 \\
PER_2 & 0.302 & 0.035 & -0.291 & -0.699 & 4.605 & 2.395 \\
PER_3 & $0.783 / 0.800$ & $0.022 / 0.024$ & -0.135 & -0.376 & 4.295 & 1.921 \\
PER_4 & $0.760 / 0.778$ & $0.022 / 0.024$ & -0.067 & -0.459 & 4.190 & 1.903 \\
\hline
\end{tabular}

Note. Item numbers, in the first column, correspond to those in the Grit-S Scale (Duckworth \& Quinn, 2009). CON = consistency of interest; PER = perseverance of effort. All the italicized items were reverse scored before the analysis. The values in italics correspond to the model with five indicators.

The 2-factor CFA models for consistency of interest and perseverance of effort. The 2-factor model with four indicators of consistency of interest and four indicators of perseverance of effort had a good fit to the data: Chi-square $(19, N=886)=70.256, p<.001$; $\mathrm{CFI}=0.953 ; \mathrm{TLI}=0.930 ; \mathrm{RMSEA}=0.055$, with the $90 \% \mathrm{CI}$ for RMSEA being $[0.042$, 0.069]. The standardized parameter estimates associated with this model are reported in Table 17. Most of the factor loadings were statistically significant and of sizeable strength. As it was the case for the corresponding 1-factor analysis reported before, CON_2, PER_1 and PER_2 had loadings that were below 0.500. The two factors had a moderate positive 
correlation (i.e., $r=0.512 ; p<.001$ ).

The 2-factor model involving the three strongest indicators of consistency of interest and the two strongest indicators of perseverance of effort had an excellent fit to the data: Chisquare $(4, N=886)=3.676, p=.452 ; \mathrm{CFI}=1.000 ; \mathrm{TLI}=1.000 ; \mathrm{RMSEA}=0.000$, with the 90\% CI for RMSEA being [0.000, 0.049]. The summary of the standardized parameter estimates associated with this model shows that all of the factor loadings were statistically significant and of sizeable strength. The two factors had a moderate positive correlation (i.e., $r=0.524 ; p<.001)$.

In sum, the findings suggest that in this population, the 1-factor models of grit did not fit well the empirical data. In contrast, a 2-factor structure of grit, which identified two facets, that is, consistency of interest and perseverance of effort, fitted the data well. Moreover, the three strongest indicators of consistency of interest and the two strongest indicators of perseverance of effort were consistently identified in all the analyses conducted. As a result, in all subsequent analyses, I worked with the two facets of grit, as measured by the three strongest indicators of consistency of interest and the two strongest indicators of perseverance of effort.

Table 17

Maximum-Likelihood Estimates of 2-Factor CFA Standardized Loadings and Standard Errors of Consistency of Interest and Perseverance of Effort Items

\begin{tabular}{cccc}
\hline Item & Scale & $\begin{array}{c}\text { Standardized factor } \\
\text { loading }\end{array}$ & Standard error \\
\hline CON_1 & CON & $0.582 / 0.575$ & $0.035 / 0.037$ \\
CON_2 & CON & 0.341 & 0.039 \\
CON_3 & CON & $0.618 / 0.605$ & $0.035 / 0.038$ \\
CON_4 & CON & $0.490 / 0.484$ & $0.036 / 0.038$ \\
PER_1 & PER & 0.365 & 0.034 \\
PER_2 & PER & 0.290 & 0.035 \\
PER_3 & PER & $0.813 / 0.822$ & $0.024 / 0.036$ \\
PER_4 & PER & $0.779 / 0.781$ & $0.024 / 0.035$ \\
\hline
\end{tabular}

Note. Item numbers, in the first column, correspond to those in the Grit-S Scale (Duckworth \& Quinn, 2009). CON = consistency of interest; PER = perseverance of effort. The items of consistency of interest were reverse scored before the analysis. The italicized values correspond to the second 2-factor CFA model, which included three indicators of consistency of interest and two measures of perseverance of effort.

CFA models for theories of intelligence. For the items of the entity theory of intelligence, the skew ranged between 0.250 and 0.468 ; their kurtosis ranged between -0.846 and -0.670 . For the items of the incremental theory of intelligence, the skew ranged between 
0.162 and 0.310 ; their kurtosis ranged between -0.576 and -0.206 . Hence, there were no problematic violations of the MVN for any of the theory of intelligence items (Curran et al., 1996).

The 1-factor CFA model for entity theory of intelligence: 4 indicators. This model had an excellent fit to the data: Chi-square $(2, N=866)=2.042, p=.360 ; \mathrm{CFI}=1.000 ; \mathrm{TLI}=$ 1.000; RMSEA $=0.005$, with the $90 \%$ CI for RMSEA being [0.000, 0.067]. The results summarized in Table 18 reveal that all the items had standardized factor loadings exceeding 0.500 and, thus, had very strong associations with the construct they were designed to measure.

Table 18

Maximum-Likelihood Estimates of Descriptive Statistics and 1-Factor CFA Standardized Loadings and Standard Errors of Entity Theory of Intelligence Items

\begin{tabular}{lcccccc}
\hline Item & $\begin{array}{c}\text { Standardized } \\
\text { factor loading }\end{array}$ & $\begin{array}{c}\text { Standard } \\
\text { error }\end{array}$ & Skew & Kurtosis & $\begin{array}{c}\text { Mean } \\
\text { item }\end{array}$ & $\begin{array}{c}\text { Variance } \\
\text { item }\end{array}$ \\
\hline EN_1 & 0.757 & 0.016 & 0.468 & -0.725 & 3.181 & 2.875 \\
EN_2 & 0.856 & 0.012 & 0.255 & -0.818 & 3.630 & 2.825 \\
EN_3 & 0.847 & 0.012 & 0.280 & -0.846 & 3.536 & 3.023 \\
EN_4 & 0.868 & 0.011 & 0.250 & -0.670 & 3.756 & 2.480 \\
\hline
\end{tabular}

Note. Item numbers, in the first column, correspond to those in the Theories of Intelligence Scale (Dweck, 1999). EN = entity theory of intelligence.

The 1-factor CFA models for incremental theory of intelligence: 4 indicators. The model with four indicators had a poor fit to the data: Chi-square $(2, N=866)=94.477, p$ $<.001 ; \mathrm{CFI}=0.956 ; \mathrm{TLI}=0.867 ; \mathrm{RMSEA}=0.228$, with the $90 \% \mathrm{CI}$ for RMSEA being [0.190, 0.269]. The results summarized in Table 19 reveal that all the items had standardized factor loadings exceeding 0.500 and, thus, had very strong associations with the construct they were designed to measure. However, an examination of the modification indices reveals that the largest modification index corresponded to an unmodeled residual covariance between IN_2 and IN_3. I estimated this parameter in the following model.

The model with four indicators and a residual covariance between IN_2 and IN_3 fitted the data very well: Chi-square $(1, N=866)=2.608, p=.106 ; \mathrm{CFI}=0.999 ; \mathrm{TLI}=0.995$; RMSEA $=0.043$, with the $90 \%$ CI for RMSEA being [0.000, 0.110]. As reported in Table 19, all the items had standardized factor loadings above 0.500, thus suggesting they are strong indicators of the construct they are designed to measure. 
Table 19

Maximum-Likelihood Estimates of Descriptive Statistics and 1-Factor CFA Standardized

Loadings and Standard Errors of Incremental Theory of Intelligence Items

\begin{tabular}{lcccccc}
\hline Item & $\begin{array}{c}\text { Standardized } \\
\text { factor loading }\end{array}$ & $\begin{array}{c}\text { Standard } \\
\text { error }\end{array}$ & Skew & Kurtosis & $\begin{array}{c}\text { Mean } \\
\text { item }\end{array}$ & $\begin{array}{c}\text { Variance } \\
\text { item }\end{array}$ \\
\hline IN_1 & $0.773 / 0.839$ & $0.017 / 0.015$ & 0.300 & -0.468 & 3.511 & 2.153 \\
IN_2 & $0.857 / 0.754$ & $0.012 / 0.019$ & 0.310 & -0.206 & 3.378 & 1.944 \\
IN_3 & $0.872 / 0.772$ & $0.021 / 0.018$ & 0.217 & -0.430 & 3.585 & 1.970 \\
IN_4 & $0.763 / 0.826$ & $0.017 / 0.016$ & 0.162 & -0.576 & 3.559 & 2.188 \\
\hline
\end{tabular}

Note. Item numbers, in the first column, correspond to those in the Theories of Intelligence Scale (Dweck, 1999). IN = incremental theory of intelligence. The italicized values correspond to the second model, which estimated the residual covariance between IN_2 and IN_3.

The 1-factor CFA models for the theories of intelligence: 8 indicators. The model with all eight indicators had a poor fit to the data: Chi-square $(20, N=866)=1089.700, p$ $<.001 ; \mathrm{CFI}=0.772 ; \mathrm{TLI}=0.680 ; \mathrm{RMSEA}=0.246$, with the $90 \% \mathrm{CI}$ for RMSEA being $[0.233,0.258]$. Results in Table 20 reveal that all four indicators of the entity theory of intelligence had negative loadings of strong magnitude and all four indicators of the incremental theory of intelligence had strong positive loadings.

The model with eight indicators and a residual covariance between IN_2 and IN_3 also fitted the data poorly: Chi-square $(19, N=866)=673.890, p<.001 ; \mathrm{CFI}=0.860 ; \mathrm{TLI}=$ 0.794; RMSEA $=0.197$, with the $90 \%$ CI for RMSEA being $[0.185,0.210]$. An examination of the parameter estimates associated with this model in Table 20 shows that all four indicators of the entity theory of intelligence had strong positive loadings and all four indicators of the incremental theory of intelligence had strong negative loadings. Taken together, the findings associated with these two models suggest that in this population a 1factor conceptualization of the theories of intelligence that collates both entity and incremental theories is not supported by the data. 
Table 20

Maximum-Likelihood Estimates of Descriptive Statistics and 1-Factor CFA Standardized Loadings and Standard Errors of the Theory of Intelligence Items (i.e., Both Entity and Incremental Theory of Intelligence Items)

\begin{tabular}{lll}
\hline Item & $\begin{array}{c}\text { Standardized factor } \\
\text { loading }\end{array}$ & $\begin{array}{c}\text { Standard } \\
\text { error }\end{array}$ \\
\hline EN_1 & $-0.713 / 0.741$ & $0.019 / 0.017$ \\
EN_2 & $-0.812 / 0.844$ & $0.015 / 0.012$ \\
EN_3 & $-0.794 / 0.829$ & $0.016 / 0.013$ \\
EN_4 & $-0.827 / 0.857$ & $0.014 / 0.011$ \\
IN_1 & $0.658 /-0.597$ & $0.023 / 0.024$ \\
IN_2 & $0.680 /-0.595$ & $0.022 / 0.024$ \\
IN_3 & $0.725 /-0.649$ & $0.020 / 0.022$ \\
IN_4 & $0.696 /-0.643$ & $0.021 / 0.022$ \\
\hline
\end{tabular}

Note. Item numbers, in the first column, correspond to those in the Theories of Intelligence Scale (Dweck, 1999). The italicized values correspond to the second model.

The 2-factor CFA models for entity theory of intelligence and incremental theory of

intelligence. The model with four indicators of entity theory and four indicators of incremental theory had an acceptable fit to the data: Chi-square $(19, N=886)=139.710$, $p<.001 ; \mathrm{CFI}=0.974 ; \mathrm{TLI}=0.962 ; \mathrm{RMSEA}=0.085$, with the $90 \% \mathrm{CI}$ for RMSEA being $[0.072,0.098]$. The standardized parameter estimates associated with this model are presented in Table 21. All of the factor loadings were statistically significant and of sizeable strength. The two factors had a strong negative correlation (i.e., $r=-0.665 ; p<.001$ ).

The model with four indicators of entity theory and four indicators of incremental theory as well as the residual covariance between IN_2 and IN_3 had an excellent fit to the data: Chi-square $(18, N=886)=51.219, p<.001 ; \mathrm{CFI}=0.993 ; \mathrm{TLI}=0.989 ; \mathrm{RMSEA}=$ 0.046, with the $90 \%$ CI for RMSEA being [0.031, 0.061]. The summary of the standardized parameter estimates associated with this model in Table 21 shows that all of the factor loadings were statistically significant and of sizeable strength. Moreover, the two factors had a strong negative correlation (i.e., $r=-0.676 ; p<.001$ ).

In sum, the findings suggest that this construct consists of two facets, that is, entity theory of intelligence and incremental theory of intelligence. Moreover, the four indicators of the entity theory and the four indicators of incremental theory provided appropriate measurements of these two facets in this population. Finally, entity theory of intelligence and incremental theory of intelligence had a strong negative correlation. 
Table 21

Maximum-Likelihood Estimates of 2-Factor CFA Standardized Loadings and Standard Errors of Entity Theory and Incremental Theory of Intelligence Items

\begin{tabular}{lccc}
\hline Item & Scale & Standardized factor loading & Standard error \\
\hline EN_1 & EN & $0.776 / 0.819$ & $0.016 / 0.015$ \\
EN_2 & EN & $0.846 / 0.763$ & $0.012 / 0.018$ \\
EN_3 & EN & $0.872 / 0.792$ & $0.011 / 0.017$ \\
EN_4 & EN & $0.775 / 0.829$ & $0.016 / 0.015$ \\
IN_1 & IN & $0.754 / 0.754$ & $0.016 / 0.016$ \\
IN_2 & IN & $0.857 / 0.857$ & $0.011 / 0.011$ \\
IN_3 & IN & $0.844 / 0.845$ & $0.012 / 0.012$ \\
IN_4 & IN & $0.872 / 0.870$ & $0.011 / 0.011$ \\
\hline
\end{tabular}

Note. Item numbers, in the first column, correspond to those in the Theories of Intelligence Scale (Dweck, 1999). EN = entity theory of intelligence; IN = incremental theory of intelligence. The italicized values correspond to the second 2-factor CFA model, which estimated the residual covariance between IN_2 and IN_3.

CFA models for critical thinking. The skew of critical thinking items ranged between -0.228 and 0.143 ; their kurtosis ranged between -0.690 and -0.297 . Hence, there were no problematic violations of the MVN for any of these items (Curran et al., 1996).

The model with all the five indicators had a poor fit to the data: Chi-square $(5, N=$ $866)=70.358, p<.001 ; \mathrm{CFI}=0.928 ; \mathrm{TLI}=0.857$; $\mathrm{RMSEA}=0.121$, with the $90 \% \mathrm{CI}$ for RMSEA being [0.097, 0.147]. Results in Table 22 indicate that all five indicators had strong relationships with the construct they were designed to measure. However, an examination of the modification indices suggests that an unmodeled residual covariance (between CT_3 and CT_4) affected negatively the model fit. Therefore, I estimated this parameter in the following CFA model.

The model with five indicators and a residual covariance between CT_3 and CT_4 fitted the data very well: chi-square $(4, N=886)=6.976, p=.137 ; \mathrm{CFI}=0.997$; $\mathrm{TLI}=0.992$; RMSEA $=0.029,90 \%$ CI for RMSEA being [0.000, 0.064]. The results in Table 22 show that four indicators had loadings larger than 0.500 and the other item (i.e., CT_3) had a loading that was slightly below 0.500 (i.e., 0.470). These findings suggest that all five items were strong indicators of critical thinking in this population. 
Table 22

Maximum-Likelihood Estimates of Descriptive Statistics and 1-Factor CFA Standardized

Loadings and Standard Errors of Critical Thinking Items

\begin{tabular}{lcccccc}
\hline Item & $\begin{array}{c}\text { Standardized } \\
\text { factor loading }\end{array}$ & $\begin{array}{c}\text { Standard } \\
\text { error }\end{array}$ & Skew & Kurtosis & $\begin{array}{c}\text { Mean } \\
\text { item }\end{array}$ & $\begin{array}{c}\text { Variance } \\
\text { item }\end{array}$ \\
\hline CT_1 & $0.513 / 0.540$ & $0.031 / 0.031$ & 0.143 & -0.690 & 3.911 & 2.397 \\
CT_2 & $0.699 / 0.737$ & $0.026 / 0.027$ & -0.140 & -0.528 & 4.348 & 2.078 \\
CT_3 & $0.564 / 0.470$ & $0.031 / 0.033$ & -0.110 & -0.408 & 4.257 & 1.945 \\
CT_4 & $0.637 / 0.557$ & $0.028 / 0.030$ & -0.166 & -0.297 & 4.383 & 1.744 \\
CT_5 & $0.625 / 0.647$ & $0.028 / 0.028$ & -0.228 & -0.398 & 4.511 & 1.943 \\
\hline
\end{tabular}

Note. Item numbers, in the first column, correspond to those in the Critical Thinking Scale of MSLQ (Pintrich et al., 1991). CT = critical thinking. The values in Italics correspond to the second model, which estimated the residual covariance between CT_3 and CT_4.

CFA model for effort regulation. The skew of effort regulation items ranged between 0.545 and -0.053 ; their kurtosis ranged between -.733 and -0.291 . Hence, there were no problematic violations of the MVN for any of these items (Curran et al., 1996).

The model with 4 indicators fitted the data poorly: Chi-square $(2, N=866)=52.435$, $p<.001 ; \mathrm{CFI}=0.917 ; \mathrm{TLI}=0.751 ; \mathrm{RMSEA}=0.169$, with the $90 \% \mathrm{CI}$ for RMSEA being $[0.131,0.210]$. As presented in Table 23, two items (i.e., ER_1 and ER_3) had standardized factor loadings smaller than 0.500 (i.e., 0.394 and 0.265 ), which suggest that these two items are weaker indicators of effort regulation in the sample. Because I wanted to use strong indicators of the given construct for LPA, I decided to retain only ER_2 and ER_4 for the subsequent analyses.

Table 23

Maximum-Likelihood Estimates of Descriptive Statistics and 1-thisor CFA Standardized Loadings and Standard Errors of Effort Regulation Items

\begin{tabular}{lcccccc}
\hline Item & $\begin{array}{c}\text { Standardized } \\
\text { factor loading }\end{array}$ & $\begin{array}{c}\text { Standard } \\
\text { error }\end{array}$ & Skew & Kurtosis & $\begin{array}{c}\text { Mean } \\
\text { item }\end{array}$ & $\begin{array}{c}\text { Variance } \\
\text { item }\end{array}$ \\
\hline$E R \_1$ & 0.394 & 0.034 & -0.053 & -0.723 & 4.164 & 2.342 \\
ER_2 & 0.724 & 0.030 & -0.120 & -0.733 & 4.265 & 2.459 \\
$E R \_3$ & 0.265 & 0.036 & -0.545 & -0.291 & 4.887 & 2.093 \\
ER_4 & 0.831 & 0.032 & -0.126 & -0.687 & 4.248 & 2.381 \\
\hline
\end{tabular}

Note. Item numbers, in the first column, correspond to those in the Effort Regulation Scale of MSLQ (Pintrich et al., 1991). ER = effort regulation. The italicized items were reverse scored before the analysis. 


\section{Results for LPA}

This section reports the results of the latent profile analyses (LPAs) conducted to identify motivation profiles that account parsimoniously for variability in the interrelationships among key motivation orientations. LPAs were conducted in Mplus, version 8 (Muthén \& Muthén, 2017). Four indicators (i.e., assessment, locomotion promotion, and prevention) were used in the LPAs; as it was the case in previous research, each indicator was calculated by averaging the corresponding scores of the assessment, locomotion, promotion, and prevention items (Harring \& Hodis, 2016; Hodis et al., 2017). As detailed in the section describing the results of the CFA analyses, I employed seven assessment items, nine locomotion items, six promotion items, and four prevention items, which were identified as strong measures of these four constructs in the corresponding CFAs. For the four indicators, their absolute values of skewness and kurtosis were below 1.119 and 0.724 ; these values are lower than the values that would indicate potential violations of multivariate normality (i.e., 2.00 for skewness and 7.00 for kurtosis; Curran et al., 1996).

In all models analysed in this research, the class-specific means and variances of the indicators as well as the latent class proportions (i.e., the proportions of individuals grouped in each class) were freely estimated. However, covariances among the indicators within each class were fixed at zero. This decision is consistent with the assumption of local independence in the LPA. Specifically, under the assumption that the analysis identifies the correct number of latent lasses, the local independence states that the indicators are uncorrelated within groups (Pastor \& Gagné, 2013; Peugh \& Fan, 2013; Tein et al., 2013). Moreover, previous LPA research conducted with regards to these constructs also restricted to zero within-class indicator covariances (Harring \& Hodis, 2016; Hodis et al., 2017).

Latent class enumeration. Determining the number of latent classes was an iterative process. Specifically, I began with a 2-class model and continued to test models with successively larger numbers of classes (i.e., three to eight classes). To avoid the potential problem of converging on local likelihood maxima, I instructed Mplus to use 2,500 random sets of starting values for all models. After 100 iterations, which are recommended for a thorough investigation (Muthén \& Muthén, 2017), the 100 best sets of starting values identified by the highest likelihood values were selected for final optimization. In all models, the best log likelihood value was replicated many times. Therefore, I am confident that the enumeration procedure converged on the global rather than local likelihood maxima. 
After all models with an increasing number of classes were estimated, a combination of statistical indexes were used to explore which model was most appropriate. Consistent with both methodological recommendation and recent substantive research (Harring \& Hodis, 2016; Hodis et al., 2017), three information criteria (i.e., AIC, BIC, and SABIC), two likelihood-ratio tests (i.e., adjusted LRT and BLRT), and the entropy index were employed. As mentioned above, for all three information criteria, smaller values suggest better model fit. For the adjusted LRT (ALRT) and BLRT tests statistics, if the $p$-values associated with these tests are lower than the significance level (i.e., 0.05), the tests suggests favouring the k-class model over the k-1 class model. Larger values of entropy (i.e., exceeding .70) indicate a more accurate separation of the classes. Besides, the sample size of each class was also considered in that models that had one or more small classes (i.e., classes having sample sizes lower than 15) were eliminated; for a similar approach see Harring and Hodis (2016) and Hodis et al. (2017).

Table 24 reports the statistical information for models with two to eight latent classes. Results presented in Table 24 show that the 8 -class model involved a class with a sample size of six. Therefore, in line with methodological recommendations (Harring \& Hodis, 2016; Hodis et al., 2017), this model was removed from consideration. Moreover, findings in Table 24 indicate that AIC did not offer useful information to determine the number of the latent classes; its values decreased as the numbers of classes increased from two to eight. BIC and ALRT suggested that the 5-class model offered the best fit. BLRT suggested selecting the 6class model. SABIC suggested adopting the 7-class solution. However, methodological research by Nylund et al. (2007) suggests that SABIC tends to generally overestimate the number of classes in mixture analyses. Therefore, the 5-class and 6-class models were retained for further consideration. For the two retained models, the value of entropy was higher for the 5-class model than the 6-class one. In addition, as illustrated in Figure 5 and Figure 6, an examination of the graphic representations of the latent profiles associated with these two models shows that with exception of the Class 6 in the 6-class model, the profiles underlying Classes 1, 2, 3, 4, and 5 were virtually identical in the two models. The latent profile associated with Class 6 in the 6-class model was parallel to that of Class 1 in the same model, but only had slightly lower values on the indicators. Therefore, Class 6 was not qualitatively distinct from to Class 1 and did not need to be identified separately. Taking together all of this information, the 5-class model offered an optimal representation of the data. 
Description of the 5-class model. In this section, each of the latent classes in the 5class model are described with regards to the patterns of endorsing assessment, locomotion, promotion, and prevention in each class. The mean motivation profiles corresponding to these five classes are presented in Figure 4; their descriptive statistics are summarized in Table 25. The values of the indicators represented in Figure 4 are average values calculated for all respondents classified in the given latent class.

Class 1. The first latent class comprised $37.70 \%$ of the students; this was the largest profile in the entire sample. The motivation profile underlying Class 1 is characterized by having a combination of high/very high values on all four indicators. Moreover, the strengths of assessment, locomotion, and promotion in Class 1 were comparable, whereas prevention was the strongest of all four orientations. For this class, locomotion and promotion were of the strongest magnitude in the entire sample.

Class 2. The second latent class consisted of $36.57 \%$ of the sample. The Class 2 profile is characterized by comparable levels of average assessment, locomotion, and promotion (which are all slightly below the average, i.e., below the corresponding mean values for the entire sample), as well as very high levels of prevention. For this class, prevention dominated in magnitude the other motivation orientations and was the strongest among all five classes.

Class 3. The third latent class comprised $8.35 \%$ of the sample. Students classified in Class 3 had strong/very strong and comparable assessment, locomotion, and promotion, but very weak prevention. For this class, assessment was the strongest and prevention the weakest among all five classes.

Class 4. The fourth latent class comprised $5.19 \%$ of the students, which was the smallest proportion in the sample. The motivation profile associated with Class 4 is characterized by having low/very low levels of assessment, locomotion, and promotion, and below average levels of prevention. In Class 4 , prevention was much stronger than any of the other motivation orientations. In addition, in this class, assessment was stronger than both locomotion and promotion; the latter dimensions were of comparable magnitudes. The average values of assessment, locomotion, and promotion in Class 4 were the weakest among all classes.

Class 5. The fifth latent class consisted of $12.19 \%$ of the sample. Class 5 profile is characterized by below average and comparable levels of assessment, locomotion, promotion, and somewhat stronger prevention (yet still below the average value of the entire sample). 
To sum up, the five identified motivation profiles have different shapes (i.e., different patterns of magnitudes of the four indicators) and appear qualitatively distinct from one another; that is, they have different patterns of absolute and relative magnitudes of the four indicators). These five motivation profiles provide a parsimonious account of the complex patterns of interrelationships among assessment, locomotion, promotion, and prevention in this population. In addition, there was considerable variability in average indicator values across the five classes (See Figure 4 and Table 25). Hence, assessment, locomotion, promotion, and prevention all made important contributions to distinguishing among these profiles. 
Table 24

Statistical Criteria Associated with Latent Class Enumeration

\begin{tabular}{ccccccccccc}
\hline Model & SSS & LL & $\begin{array}{c}\text { No. } \\
\text { Parameters }\end{array}$ & AIC & BIC & SABIC & $p$-ALRT & $p$-BLRT & Entropy \\
\hline 2-class & 439 & -4734.531 & 17 & 9503.062 & 9584.436 & 9530.448 & $<.01$ & $<.01$ & 0.631 \\
3-class & 140 & -4605.195 & 26 & 9262.390 & 9386.844 & 9304.273 & $<.01$ & $<.01$ & $\mathbf{0 . 7 5 8}$ \\
4-class & 95 & -4537.828 & 35 & 9145.657 & 9313.192 & 9202.039 & $<.01$ & $<.01$ & 0.727 \\
5-class & 46 & -4506.363 & 44 & 9100.727 & $\mathbf{9 3 1 1 . 3 4 2}$ & 9171.607 & $<.01$ & $<.01$ & 0.731 \\
6-class & 36 & -4476.661 & 53 & 9059.332 & 9313.018 & 9144.701 & 0.33 & $<.01$ & 0.695 \\
7-class & 17 & -4458.074 & 62 & 9040.148 & 9336.925 & $\mathbf{9 1 4 0 . 0 2 5}$ & 0.829 & 0.406 & 0.729 \\
8-class & 6 & -4441.915 & 71 & $\mathbf{9 0 2 5 . 8 3 0}$ & 9365.687 & 9140.205 & NA & NA & 0.718 \\
\hline
\end{tabular}

Note. SSS = the smallest sample size associated with any of the classes identified by the given model; LL = log-likelihood; AIC = Akaike Information Criterion; BIC=Bayesian Information Criterion; SABIC $=$ sample-size adjusted BIC; $p$-ALRT $=$ the $p$-value associated with the LoMendell-Rubin adjusted likelihood ratio test; $p$-BLRT $=$ the $p$-value associated with the bootstrapped likelihood ratio test; NA = the given value was not calculated because it pertains to a model with small sample sizes that was excluded from consideration. Values in bolds denote that the respective index suggests extracting a model with the specific number of classes. 


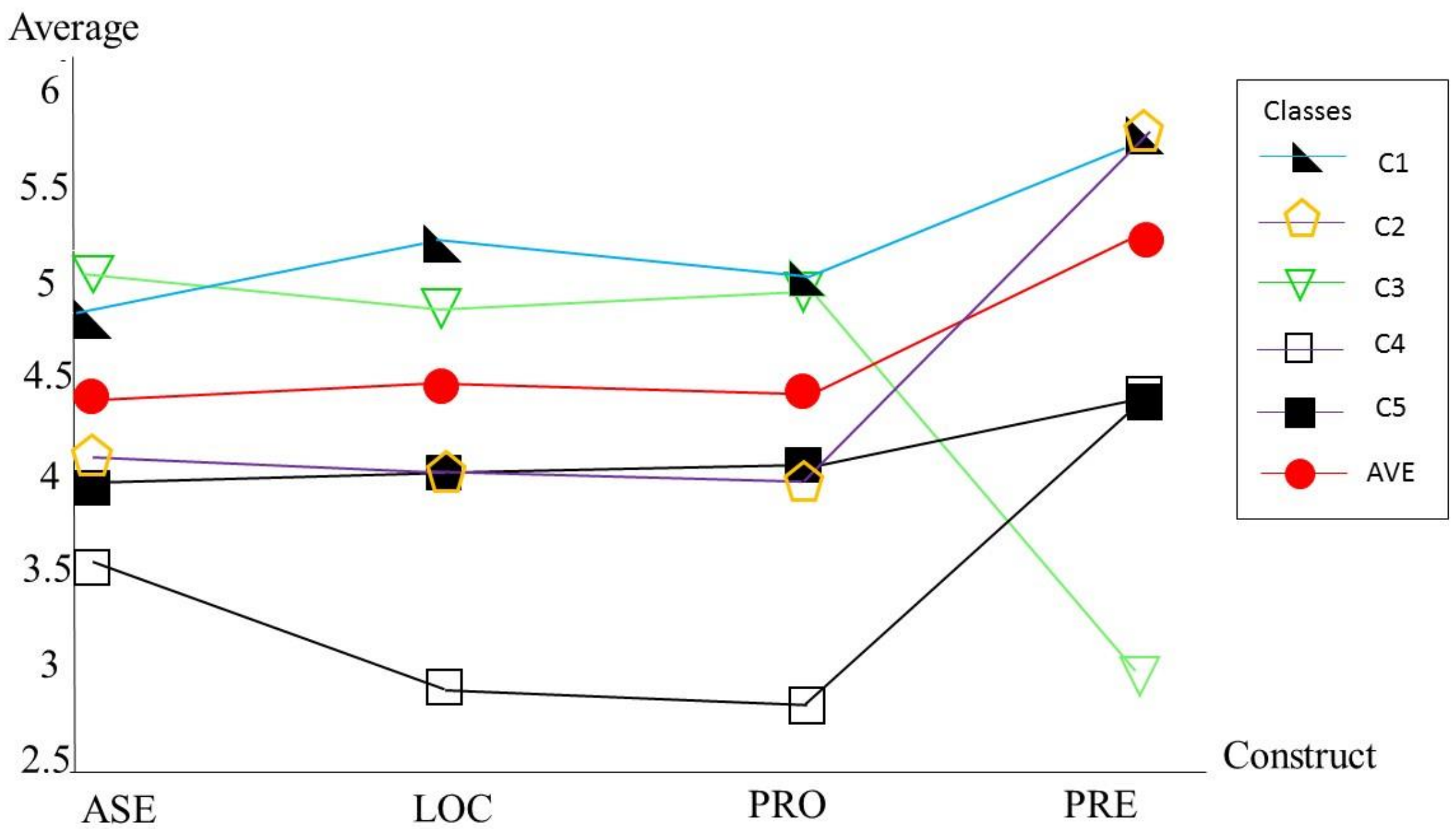

Figure 5. The model with five latent classes (i.e., five motivation profiles).

Note $. \mathrm{ASE}=$ assessment $; \mathrm{LOC}=$ locomotion $; \mathrm{PRO}=$ promotion $; \mathrm{PRE}=$ prevention $; \mathrm{AVE}=$ average across the entire sample. 


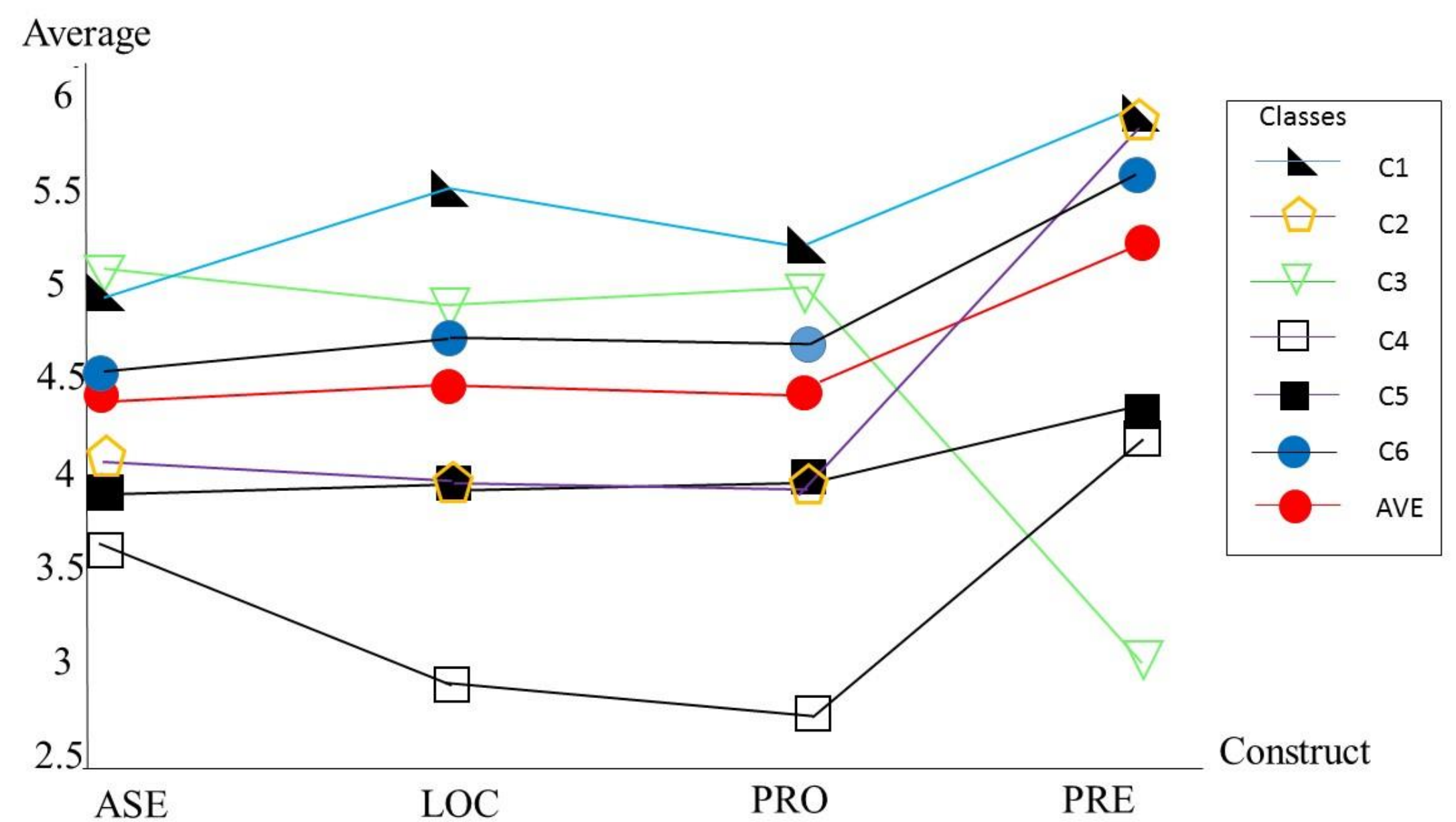

Figure 6. The model with six latent classes (i.e., six motivation profiles).

Note. $\mathrm{ASE}=$ assessment $\mathrm{LOC}=$ locomotion; $\mathrm{PRO}=$ promotion; $\mathrm{PRE}=$ prevention; $\mathrm{AVE}=$ average across the entire sample. 
Table 25

Descriptive Statistics for Classes and Total Samples of the Five-class Model

\begin{tabular}{cccccccccc}
\hline Latent class & $\%$ & \multicolumn{2}{c}{ Assessment } & \multicolumn{2}{c}{ Locomotion } & \multicolumn{2}{c}{ Promotion } & \multicolumn{2}{c}{ Prevention } \\
\cline { 2 - 9 } & & $\mathrm{M}$ & Var & $\mathrm{M}$ & Var & $\mathrm{M}$ & $\mathrm{Var}$ & $\mathrm{M}$ & Var \\
\hline Class 1 & 37.70 & 4.829 & 0.775 & 5.228 & 0.344 & 5.090 & 0.397 & 5.846 & 0.419 \\
Class 2 & 36.57 & 4.183 & 0.757 & 4.120 & 0.355 & 4.048 & 0.477 & 5.864 & 0.266 \\
Class 3 & 8.35 & 5.145 & 0.753 & 4.924 & 0.516 & 5.057 & 0.519 & 3.059 & 0.561 \\
Class 4 & 5.19 & 3.659 & 1.454 & 2.981 & 0.360 & 2.865 & 0.359 & 4.484 & 2.061 \\
Class 5 & 12.19 & 4.018 & 0.512 & 4.080 & 0.224 & 4.091 & 0.216 & 4.423 & 0.926 \\
Total sample & 100 & 4.446 & 0.976 & 4.517 & 0.875 & 4.448 & 0.901 & 5.330 & 1.161 \\
\hline
\end{tabular}


In the following section, I compare and contrast the motivation profiles identified in my research (i.e., in a population of Chinese undergraduates) and the profiles identified in a population of secondary-school students from New Zealand (Harring \& Hodis, 2016; Hodis et al., 2017). Using data collected from samples of New Zealand secondary-school students, Harring and Hodis (2016) and Study 1 in Hodis et al. (2017; Study 1) both identified six motivation profiles; Study 2 in Hodis et al. (2017) identified seven profiles. Because the corresponding profiles identified in Harring and Hodis (2016) and the two studies reported by Hodis et al. (2017) were similar for all classes in terms of shape, absolute and relative magnitudes of all four indicators, I compare the five motivation profiles in my research to the six profiles identified in Study 1 by Hodis et al. (2017; see Figure 3, p. 186 in this source). I did not find any profile in Hodis et al. (2017) similar to the C2 profile identified in my research; therefore, in this section, I discuss similarities and differences between the remaining four profiles in my research and the corresponding profiles uncovered by Hodis et al. (2017).

The motivation profiles corresponding to $\mathrm{C} 1$ in my research and $\mathrm{C} 6$ in Hodis et al. (2017) were both characterized by a combination of high/very high mean levels on promotion, locomotion, and prevention. However, these two profiles differed in both relative and absolute magnitudes of the four indicators. Specifically, in the $\mathrm{C} 1$ profile identified in my research, prevention was the strongest of all four dimensions; the average values of assessment, locomotion, and promotion were lower than the mean value of prevention and the strength of these three indicators were comparable in C1. In contrast, in C6 in Hodis et al. (2017), the mean level of prevention was somewhat weaker than those of locomotion and promotion, although it had the highest average value of all six classes identified in the Hodis et al. (2017) study. Moreover, in the C6 profile identified by Hodis et al. (2017), the mean value of assessment, which was slightly below the average for the entire sample, was much weaker than the other dimensions. In addition, $\mathrm{C} 1$ in my research had stronger mean levels of assessment and prevention, but weaker mean levels of locomotion and promotion than C6 in Hodis et al. (2017).

The C3 motivation profile in my research had a similar shape to the $\mathrm{C} 4$ profile in Hodis et al. (2017). Both profiles were characterized by high levels of assessment, locomotion, and promotion, but very low levels of prevention. In addition, both profiles had the strongest assessment and the weakest prevention of all classes in each respective sample. Moreover, the two profiles were similar with regard to the fact that the magnitudes of assessment, 
locomotion, and promotion were comparable in both C3 (my research) and C6 (Hodis et al.' study). Specifically, in the C3 profile identified in my research, all these three indicators had comparable mean levels. In contrast, in C4 in Hodis et al. (2017), promotion was somewhat stronger than the other two dimensions. However, the two profiles differed in the absolute magnitudes of all indicators. In particular, C3 in my research had a stronger mean level of prevention, but lower average scores of assessment, locomotion, and promotion than $\mathrm{C} 4$ in Hodis et al. (2017). These differences are consistent with cross-cultural research involving regulatory focus and regulatory mode orientations (Higgins, 2008; Higgins, Pierro, \& Kruglanski, 2008). I will offer additional details at the end of this section.

The $\mathrm{C} 4$ motivation profile in my research and the $\mathrm{C} 2$ motivation profile in Hodis et al. (2017) were both characterized by low/very low levels on assessment, locomotion, promotion, and prevention. Moreover, in both of these profiles, average values of locomotion and promotion were the weakest among all the classes in each sample; the two profiles had similar average values of assessment and locomotion. In addition, in both cases, prevention had the strongest mean value of all dimensions in the respective class. The two profiles had some differences in the relative strengths of all indicators. Specifically, in C4 in my research, the mean differences between prevention (on the one hand) and locomotion and promotion (on the other hand) were somewhat stronger than the corresponding differences in the $\mathrm{C} 2$ profile. Again, this is consistent with cross-cultural research on prevention (Higgins, 2008; Higgins et al., 2008). However, differences between prevention and assessment were comparable in the two studies.

The motivation profile associated with C5 in my research was similar to the profile underlying C1 in Hodis et al. (2017). Specifically, both profiles were characterized by below average and comparable levels on all indicators and no dimension dominated the others in magnitude. In addition, average values of assessment in the two profiles were virtually identical. The two profiles differed with respect to the rank order of promotion and prevention. Specifically, prevention was somewhat stronger than promotion in the C5 profile identified in my research, whereas this rank order was reversed in the $\mathrm{C} 1$ profile in Hodis et al. (2017). Moreover, C5 in my research had a somewhat higher mean level of prevention, but lower mean levels of locomotion and promotion than C1 in Hodis et al. (2017). Again, these results are in line with cross-cultural findings (Higgins, 2008; Higgins et al., 2008). 
In sum, although more latent classes were identified in Hodis et al. (2017) than in my research (i.e., six versus five, respectively), four motivation profiles (i.e., all profiles except C2) in my research had structurally similar counterparts in Hodis et al. (2017). Particularly, the $\mathrm{C} 3$ and $\mathrm{C} 4$ profiles identified in my research were very similar in shape with the $\mathrm{C} 4$ and C2 profiles identified in Hodis et al. (2017). However, there are also some differences that need to be noted among the corresponding profiles in the two studies. Most of the interprofile differences arise from a stronger prevention focus, a somewhat weaker promotion focus and a somewhat stronger assessment orientation in the sample of Chinese undergraduate students compared to the sample of secondary school students from New Zealand. These differences are consistent with the findings of cross-cultural studies (Higgins, 2008; Higgins et al., 2008). These studies reported that individuals from Asian countries (e.g., Japan) had stronger prevention and assessment orientations and a weaker promotion focus compared to their counterparts from western countries (e.g., the US and Italy; Higgins, 2008; Higgins et al., 2008).

\section{Comparison of Motivation Profiles on Criteria of Interest}

This section examines similarities and differences among the five motivation profiles identified in this research with regard to eight criteria (i.e., consistency of interest, perseverance of effort, incremental theory of intelligence, entity theory of intelligence, critical thinking, Cognitive Reflection Test, effort regulation, and evaluations/perceptions of academic success). The maximum score for the Cognitive Reflection Test was three, the perceptions of academic success criterion were rated on a 1-100 scale; all the other criteria were recorded on 7-point Likert-style scales. For each criterion, tests of significance for the equality of means across these profiles were conducted. To do so, the three-step approach recommended by Asparouhov and Muthén (2014) was used in Mplus, version 8 (Muthén \& Muthén, 2017). Specifically, first, the latent profile model was estimated using the four profile indicators. Then the measurement error for the most likely latent class membership was determined. Based on the findings from the first two steps, the final step estimated the distal outcome model which evaluated differences among the five classes in terms of the criteria of interest in this research.

In Mplus, the three-step procedure was conducted "automatically using the AUXILIARY option of the VARIABLE command" (Asparouhov \& Muthén, 2014, p. 332). I specified DU3STEP as the AUXILIARY command for all eight criteria. Using DU3STEP, the 
criterion variables were treated as having unequal means and variances. However, this command did not work for three criteria (i.e., entity theory of intelligence, critical thinking, and Cognitive Reflection Test). Then, I specified DE3STEP as the AUXILIARY command for these three criteria, which constrained variances to be equal across classes. I found that this command still did not work for entity theory of intelligence. Therefore, I had to calculate scores for entity theory of intelligence (by averaging corresponding items) and then used the 1-way ANOVA in SPSS to test mean differences for entity theory of intelligence across classes. The results of the test statistics are presented in Table 26 and Table 27. Tables 28 to 35 report the effect sizes associated with mean differences among the five profiles for each criterion in part. Following I overview these results, beginning with those corresponding to grit.

Grit. This criterion measured students' tendency to focus on their long-term goals and persevere in spite of obstacles. Grit consists of two dimensions, namely, consistency of interest and perseverance of effort.

Consistency of interest. Results of tests of significance presented in Table 26 show that the highest mean level of consistency of interest was associated with the $\mathrm{C} 1$ motivation profile; this profile was characterized by strong/very strong levels on all four motivation orientations. Students classified in C1 had a significantly larger average value of consistency of interest than their counterparts classified in any of the other classes; the corresponding effect sizes were moderate or large (see Table 28 for effect sizes associated with this criterion).

For the other classes, mean levels of consistency of interest were lower than the average for the entire sample. Students in C2 had somewhat higher levels of this criterion than their counterparts in C5 and C3. However, these differences were generally small and not statistically significant (see Table 28).

The lowest mean level of consistency of interest was associated with the $\mathrm{C} 4$ profile, which was characterized by low/very low levels on all four motivation orientations. On average, students classified in C4 and C3 did not differ significantly with regard to this criterion; however, differences between $\mathrm{C} 4$ and the other classes were statistically significant and medium to large in magnitude (see Table 28).

Students classified in the C5 profile had average/below average and relatively comparable levels on assessment, locomotion, promotion, and prevention. The mean level of consistency of interest in this class was only slightly below the average of the whole sample. C5 had significantly larger values of this criterion than C4 and significantly smaller values 
than $\mathrm{C} 1$; these differences were of medium magnitudes (see Table 28).

Perseverance of effort. Results reported in Table 26 indicate that the average values of perseverance of effort were largest for motivation profiles that were characterized by high levels on assessment, locomotion, and promotion (i.e., C1 and C3). Interestingly, these two profiles differed markedly with regard to prevention: prevention was very high in $\mathrm{C} 1$ and very low in C3. Students in C1 had significantly larger average values of perseverance of effort than their counterparts in other classes; these differences were moderate or large/very large in magnitude. However, students in C3 did not differ significantly from their counterparts in $\mathrm{C} 2$ and $\mathrm{C} 5$ with regard to perseverance of effort; the corresponding effect sizes were also small (see Table 29 for effect sizes associated with this criterion).

The lowest mean levels of perseverance of effort was associated with the $\mathrm{C} 4$ profile; this profile was characterized by low/very low levels on all motivation orientations. Students classified in $\mathrm{C} 4$ had a significantly lower mean level of this criterion than their counterparts in other classes; these differences were of large/very large magnitudes (see Table 29).

The average level of perseverance of effort associated with $\mathrm{C} 5$ was somewhat smaller than the average value of this criterion for the entire sample. Notably, students in C5, C2, and C3 did not differ significantly from one another with regard to average levels of perseverance of effort (see Table 29). 
Table 26

Descriptive Statistics and the Results of the Tests of Significance for Differences among Classes with Regard to Grit and Theories of Intelligence

\begin{tabular}{|c|c|c|c|c|c|c|c|c|c|}
\hline \multirow[t]{3}{*}{ Latent class } & \multirow[t]{3}{*}{$\%$} & \multicolumn{4}{|c|}{ Grit } & \multicolumn{4}{|c|}{ Theories of intelligence } \\
\hline & & \multicolumn{2}{|c|}{$\mathrm{CON}$} & \multicolumn{2}{|c|}{ PER } & \multicolumn{2}{|c|}{ IN } & \multicolumn{2}{|c|}{$\mathrm{EN}$} \\
\hline & & $\mathrm{M}$ & SE & $\mathrm{M}$ & SE & $\mathrm{M}$ & SE & $\mathrm{M}$ & SE \\
\hline 1 & 37.70 & 4.285 & 0.073 & 5.015 & 0.072 & $3.321^{\mathrm{a}}$ & 0.107 & $3.360^{\mathrm{a}}$ & 0.085 \\
\hline 2 & 36.57 & $3.878^{\mathrm{a}}$ & 0.082 & $3.872^{\mathrm{a}}$ & 0.085 & $3.705^{b c}$ & 0.116 & $3.654^{\mathrm{ab}}$ & 0.079 \\
\hline 3 & 8.35 & $3.616^{\mathrm{ab}}$ & 0.153 & $4.145^{\mathrm{a}}$ & 0.188 & $3.333^{\mathrm{ab}}$ & 0.196 & $3.324^{\mathrm{ab}}$ & 0.176 \\
\hline 4 & 5.19 & $3.259^{\mathrm{b}}$ & 0.194 & 2.768 & 0.310 & $4.124^{\mathrm{c}}$ & 0.241 & $4.054^{\mathrm{b}}$ & 0.209 \\
\hline 5 & 12.19 & $3.852^{\mathrm{a}}$ & 0.126 & $3.874^{\mathrm{a}}$ & 0.139 & $3.469^{\mathrm{ab}}$ & 0.200 & $3.565^{\mathrm{ab}}$ & 0.120 \\
\hline Total sample & 100 & 3.962 & 1.132 & 4.242 & 1.253 & 3.526 & 1.466 & 3.526 & 1.467 \\
\hline
\end{tabular}

Note. CON= consistency of interest; PER = perseverance of effort; IN = incremental theory of intelligence; EN = entity theory of intelligence; $\mathrm{M}=$ mean; $\mathrm{SE}$ = standard error; The tests of significance for EN were conducted in SPSS; For the total sample, standard deviations are reported instead of standard errors; Mean values in a given column that share the same superscript do not differ significantly at the 0.05 level of significance. 
Table 27

Descriptive Statistics and the Results of the Tests of Significance for Differences among Classes with regard to Critical Thinking, Effort Regulation, and Perceptions/Evaluations of Academic Success

\begin{tabular}{|c|c|c|c|c|c|c|c|c|c|}
\hline \multirow{3}{*}{$\begin{array}{l}\text { Latent } \\
\text { class }\end{array}$} & \multirow[t]{3}{*}{$\%$} & \multicolumn{4}{|c|}{ Critical thinking } & \multirow{2}{*}{\multicolumn{2}{|c|}{ Effort regulation }} & \multirow{2}{*}{\multicolumn{2}{|c|}{$\begin{array}{c}\text { Perceptions/evaluations of } \\
\text { academic success }\end{array}$}} \\
\hline & & \multicolumn{2}{|c|}{$\mathrm{CT}$} & \multicolumn{2}{|c|}{ CRT } & & & & \\
\hline & & $\mathrm{M}$ & SE & $\mathrm{M}$ & $\mathrm{SE}$ & M & $\mathrm{SE}$ & M & $\mathrm{SE}$ \\
\hline 1 & 37.70 & 3.914 & 0.086 & $2.640^{\mathrm{a}}$ & 0.039 & $4.877^{a}$ & 0.056 & $79.151^{\mathrm{ab}}$ & 1.305 \\
\hline 2 & 36.57 & $2.953^{\mathrm{a}}$ & 0.101 & $2.728^{a}$ & 0.040 & 3.723 & 0.064 & $79.651^{\mathrm{a}}$ & 0.951 \\
\hline 3 & 8.35 & 4.320 & 0.164 & $2.614^{\mathrm{a}}$ & 0.086 & $4.965^{\mathrm{a}}$ & 0.101 & $73.174^{\text {bd }}$ & 2.673 \\
\hline 4 & 5.19 & $3.002^{\mathrm{a}}$ & 0.221 & $2.532^{\mathrm{a}}$ & 0.135 & 3.232 & 0.182 & $68.342^{\mathrm{cd}}$ & 3.346 \\
\hline 5 & 12.19 & 3.571 & 0.120 & $2.572^{\mathrm{a}}$ & 0.100 & 4.157 & 0.077 & $62.196^{c}$ & 1.861 \\
\hline $\begin{array}{l}\text { Total } \\
\text { sample }\end{array}$ & 100 & 3.508 & 1.245 & 2.654 & 0.590 & 4.282 & 0.997 & 75.533 & 12.489 \\
\hline
\end{tabular}

Note. $\mathrm{CT}=$ critical thinking; $\mathrm{CRT}=$ Cognitive Reflection Test; $\mathrm{M}=$ mean; $\mathrm{SE}=$ standard error; For the total sample, standard deviations are reported instead of standard errors; Mean values in a given column that share the same superscript do not differ significantly at the 0.05 level of significance. 
Table 28

Cohen's d Effect Sizes Associated with Mean Differences between Classes with regard to Consistency of Interest

\begin{tabular}{llllll}
\hline Class & C1 & C2 & C3 & C4 & C5 \\
\hline C1 & NA & $0.290^{* *}$ & $0.503^{* *}$ & $0.770^{* *}$ & $0.326^{* *}$ \\
C2 & & NA & 0.181 & $0.425^{* *}$ & 0.018 \\
C3 & & NA & 0.271 & -0.180 \\
C4 & & & NA & $-0.452^{*}$ \\
C5 & & & & NA \\
\hline
\end{tabular}

Note. The sign of each effect size reflects calculating the given effect size by subtracting average values of the criterion in the larger-numbered classes from their respective counterparts in the smaller-numbered classes. For example, to obtain the effect size in row $\mathrm{C} 1$ column $\mathrm{C} 2$ the mean consistency of interest in $\mathrm{C} 2$ was subtracted from its counterpart in $\mathrm{C} 1 . * * p<.01 . * p<.05$.

Table 29

Cohen's d Effect Sizes Associated with Mean Differences between Classes with regard to Perseverance of Effort

\begin{tabular}{llllll}
\hline Class & C1 & C2 & C3 & C4 & C5 \\
\hline C1 & NA & $0.802^{* *}$ & $0.633^{* *}$ & $1.569^{*}$ & $0.846^{* *}$ \\
C2 & & NA & -0.177 & $0.685^{* *}$ & -0.001 \\
C3 & & NA & $0.758^{* *}$ & 0.179 \\
C4 & & & NA & $-0.664^{* *}$ \\
C5 & & & & NA \\
\hline
\end{tabular}

Note. The sign of each effect size reflects calculating the given effect size by subtracting average values of the criterion in the larger-numbered classes from their respective counterparts in the smaller-numbered classes. For example, to obtain the effect size in row $\mathrm{C} 1$ column $\mathrm{C} 2$ the mean perseverance of effort in $\mathrm{C} 2$ was subtracted from its counterpart in C1. $* * p<.01 . * p<.05$. 
Table 30

Cohen's d Effect Sizes Associated with Mean Differences between Classes with regard to Incremental Theory of Intelligence

\begin{tabular}{llllll}
\hline Class & C1 & C2 & C3 & C4 & C5 \\
\hline C1 & NA & $-0.190^{*}$ & -0.006 & $-0.418^{* *}$ & -0.075 \\
C2 & & NA & 0.184 & -0.206 & 0.113 \\
C3 & & NA & $-0.475^{*}$ & -0.071 \\
C4 & & & & NA & $0.335^{*}$ \\
C5 & & & & & NA \\
\hline
\end{tabular}

Note. The sign of each effect size reflects calculating the given effect size by subtracting average values of the criterion in the larger-numbered classes from their respective counterparts in the smaller-numbered classes. For example, to obtain the effect size in row $\mathrm{C} 1$ column $\mathrm{C} 2$ the mean incremental theory of intelligence in $\mathrm{C} 2$ was subtracted from its counterpart in $\mathrm{C} 1 .{ }^{* *} p<.01 .{ }^{*} p<.05$.

Table 31

Cohen's d Effect Sizes Associated with Mean Differences between Classes with regard to Entity Theory of Intelligence

\begin{tabular}{llllll}
\hline Class & C1 & C2 & C3 & C4 & C5 \\
\hline C1 & NA & -0.198 & 0.023 & $-0.454^{*}$ & -0.139 \\
C2 & & NA & 0.230 & -0.283 & 0.065 \\
C3 & & NA & -0.498 & -0.178 \\
C4 & & & NA & 0.379 \\
C5 & & & & NA \\
\hline
\end{tabular}

Note. The sign of each effect size reflects calculating the given effect size by subtracting average values of the criterion in the larger-numbered classes from their respective counterparts in the smaller-numbered classes. For example, to obtain the effect size in row $\mathrm{C} 1$ column $\mathrm{C} 2$ the mean entity theory of intelligence in $\mathrm{C} 2$ was subtracted from its counterpart in $\mathrm{C} 1$.

$* * p<.01 . * p<.05$. 
Table 32

Cohen's d Effect Sizes Associated with Mean Differences between Classes with regard to Critical Thinking

\begin{tabular}{llllll}
\hline Class & C1 & C2 & C3 & C4 & C5 \\
\hline C1 & NA & $0.566^{* *}$ & $-0.263^{*}$ & $0.583^{* *}$ & $0.229^{*}$ \\
C2 & & NA & $-0.781^{* *}$ & -0.027 & $-0.365^{* *}$ \\
C3 & & & NA & $0.912^{* *}$ & $0.569^{* *}$ \\
C4 & & & NA & $0.429^{*}$ \\
C5 & & & & NA
\end{tabular}

Note. The sign of each effect size reflects calculating the given effect size by subtracting average values of the criterion in the larger-numbered classes from their respective counterparts in the smaller-numbered classes. For example, to obtain the effect size in row $\mathrm{C} 1$ column $\mathrm{C} 2$ the mean critiacl thinking in $\mathrm{C} 2$ was subtracted from its counterpart in $\mathrm{C} 1$.

$* * p<.01 . * p<.05$.

Table 33

Cohen's d Effect Sizes Associated with Mean Differences between Classes with regard to Cognitive Reflection Test (CRT)

\begin{tabular}{llllll}
\hline Class & C1 & C2 & C3 & C4 & C5 \\
\hline C1 & NA & -0.123 & 0.036 & 0.146 & 0.085 \\
C2 & & NA & 0.158 & 0.262 & 0.192 \\
C3 & & NA & 0.101 & 0.045 \\
C4 & & & & NA & -0.040 \\
C5 & & & & & NA \\
\hline
\end{tabular}

Note. The sign of each effect size reflects calculating the given effect size by subtracting average values of the criterion in the larger-numbered classes from their respective counterparts in the smaller-numbered classes. For example, to obtain the effect size in row $\mathrm{C} 1$ column $\mathrm{C} 2$ the mean score in CRT in $\mathrm{C} 2$ was subtracted from its counterpart in $\mathrm{C} 1$.

$* * p<.01 . * p<.05$. 
Table 34

Cohen's d Effect Sizes Associated with Mean Differences between Classes with regard to Effort Regulation

\begin{tabular}{llllll}
\hline Class & C1 & C2 & C3 & C4 & C5 \\
\hline C1 & NA & $1.060^{* *}$ & -0.088 & $1.566^{* *}$ & $0.739^{* *}$ \\
C2 & & NA & $-1.124^{* *}$ & $0.422^{*}$ & $-0.404^{* *}$ \\
C3 & & & NA & $1.693^{* *}$ & $0.975^{* *}$ \\
C4 & & & & NA & $-0.974^{* *}$ \\
C5 & & & & NA \\
\hline
\end{tabular}

Note. The sign of each effect size reflects calculating the given effect size by subtracting average values of the criterion in the larger-numbered classes from their respective counterparts in the smaller-numbered classes. For example, to obtain the effect size in row $\mathrm{C} 1$ column $\mathrm{C} 2$ the mean effort regulation in $\mathrm{C} 2$ was subtracted from its counterpart in $\mathrm{C} 1$.

$* * p<.01 . * p<.05$.

Table 35

Cohen's d Effect Sizes Associated with Mean Differences between Classes with regard to Perceptions/Evaluations of Academic Success

\begin{tabular}{llllll}
\hline Class & C1 & C2 & C3 & C4 & C5 \\
\hline C1 & NA & -0.024 & 0.252 & $0.456^{* *}$ & $0.742^{* *}$ \\
C2 & & NA & $0.353^{*}$ & $0.632^{* *}$ & $0.986^{* *}$ \\
C3 & & & NA & 0.211 & $0.525^{* *}$ \\
C4 & & & & NA & 0.301 \\
C5 & & & & & NA
\end{tabular}

Note. The sign of each effect size reflects calculating the given effect size by subtracting average values of the criterion in the larger-numbered classes from their respective counterparts in the smaller-numbered classes. For example, to obtain the effect size in row $\mathrm{C} 1$ column $\mathrm{C} 2$ the mean score of academic success in $\mathrm{C} 2$ was subtracted from its counterpart in $\mathrm{C} 1$.

$* * p<.01 . * p<.05$. 
Theories of intelligence. This criterion measured students' beliefs regarding the nature of intelligence. Two different constructs capture this criterion, namely, incremental theory of intelligence and entity theory of intelligence.

Incremental theory of intelligence. Differences among the five classes were generally small with regard to this criterion (see Table 30 for effect sizes associated with this criterion). Tests of significance reported in Table 26 indicate that mean levels of incremental theory of intelligence were relatively high for the $\mathrm{C} 4$ and $\mathrm{C} 2$ motivation profiles. There were no significant differences between C4 and C2. Differences between C4 and the other classes were significant and of medium magnitudes. Differences between C2 and all other classes were either not statistically significant or small (see Table 30).

Low and similar average values of incremental theory of intelligence were associated with C1, C3, and C5. The mean level of incremental theory of intelligence associated with C5 was slightly below the average of the whole sample. Differences in this criterion among these three classes were very small and not statistically significant (see Table 30).

Entity theory of intelligence. As it was the case for incremental theory of intelligence, differences among the five classes with regard to entity theory of intelligence were generally small and, most often, not statistically significant (see Table 31 for effect sizes associated with this criterion). Again similar to the case for incremental theory of intelligence, mean levels of entity theory of intelligence were the highest for C4 and C2 (see Table 26). Notably, however, C4 did not differ significantly from C2, C3, and C5 with regard to this criterion; C2 did not differ significantly from C1, C3, and C5.

Similar to the case for the incremental theory of intelligence, students classified in C3 and C1 had the lowest (and similar) mean levels of entity theory of intelligence. These two classes did not differ significantly with regard to this criterion. Consistent with the case of incremental theory of intelligence, C5 did no differ significantly from C1 and C3 with regard to entity theory of intelligence. Students classified in $\mathrm{C} 1$ had a moderately lower mean level of this criterion than their counterparts in $\mathrm{C} 4$, but did not differ significantly from their counterparts in the remaining classes with regard to entity theory of intelligence. There were no significant differences with respect to this criterion between $\mathrm{C} 3$ and the other classes; the corresponding effect sizes were generally small to medium (see Table 31).

The mean level of entity theory of intelligence associated with C5 was slightly above the average of the whole sample. Differences in this criterion among C5 and the other classes were generally small and not statistically significant (see Table 31).

Critical thinking. This criterion rated students' tendecy to use critical thinking in 
learning. As it was the case for perseverance of effort, average values of critical thinking were the highest for the two motivation profiles that had high levels of assessment, locomotion and promotion (i.e., C3 and C1). As noted previously, C1 and C3 have markedly different levels of prevention. C3 had a moderately larger level of critical thinking than C1. Differences between these two classes and the other classes were statistically significant and of medium or large magnitudes (see Table 32 for effect sizes associated with this criterion).

The lowest mean levels of critical thinking were associated with motivation profiles that were characterized by low or below average levels of assessment, locomotion, and promotion (i.e., C2 and C4; see Table 27). C2 and C4 did not differ significantly with regard to critical thinking, but differences between these two classes and the other classes were significant and of medium or large magnitudes (see Table 321).

The mean level of critical thinking associated with C5 was slightly above the average of the whole sample. On average, critical thinking in C5 was significantly lower than in C3 and $\mathrm{C} 1$ and significantly higher than in $\mathrm{C} 2$ and $\mathrm{C} 4$; the corresponding effect sizes were small to medium (see Table 32).

Cognitive Reflection Test (CRT). There were no significant differences among these five classes with regard to this criterion (see Table 27). Consistent with this finding, the corresponding effect sizes were generally small/very small (see Table 33). In all classes, the average scores of CRT were above 2.5 on a $0-3$ scale. This finding suggests that the CRT may have been too easy for the participants, perhaps leading to a ceiling effect.

Effort regulation. This criterion gauged students' ability to regulate their effort when facing uninteresting tasks. Similar to the case for perseverance of effort and critical thinking, the highest mean levels of effort regulation were associated with $\mathrm{C} 3$ and $\mathrm{C} 1$; these two classes were characterized by strong assessment, locomotion, and promotion. As mentioned previously, C1 and C3 differed markedly with respect to prevention. Notably, there were no significant differences between C3 and C1 with regard to effort regulation. Differences in this criterion between these two classes and the others were significant and large/very large in magnitude (see Table 34 for effect sizes associated with effort regulation).

For the other classes, the mean levels of this criterion were lower than the average for the entire sample. As it was the case for critical thinking, students classified in C4 and C2 had the lowest mean levels of effort regulation (see Table 27). As noted previously, these two classes were characterized by low or below average levels of assessment, locomotion, and promotion. Notably, these two classes had significantly lower mean levels of effort regulation than the other classes; these differences were of medium or large/very large magnitudes (see 
Table 34).

For C5, the means level of effort regulation was slightly below the average of the whole sample. On average, effort regulation in C5 was significantly lower than in C1 and C3 and significantly larger than in $\mathrm{C} 2$ and $\mathrm{C} 4$; the corresponding effect sizes were medium to large (see Table 34).

Perceptions/evaluations of academic success. This criterion captured students' evaluations/perceptions of success with studies at the university (i.e., "How do you evaluate your success with your studies so far at university?" Students recorded their answers to this question on a scale from 0 to 100 , where 0 is "not at all successful" and 100 is "extremely successful"). The highest average scores of perceptions/evaluations of academic success were reported by students classified in C2 and C1 (see Table 27). There were no significant differences between these two classes with regard to this criterion. Differences between C2 and the other classes were significant and medium or large in magnitude. $\mathrm{C} 1$ did not differ significantly from $\mathrm{C} 3$ with regard to this criterion, but differences between $\mathrm{C} 1$ (on the one hand) and C4 and C5 (on the other hand) were statistically significant and of moderate magnitudes (see Table 35 for effect sizes associated with this criterion).

For the other classes, mean levels of this criterion were lower than the average for the entire sample. The lowest average score was associated with C5. C5 and C4 did not differ significantly with regard to this criterion. Differences between C5 and the other classes were significant and large/very large in magnitude. Although students classified in C3 had a larger average value of perceptions/evaluations of academic success than students classified in C4 (see Table 27), this difference was not statistically significant and was associated with a low effect size (see Table 35). 


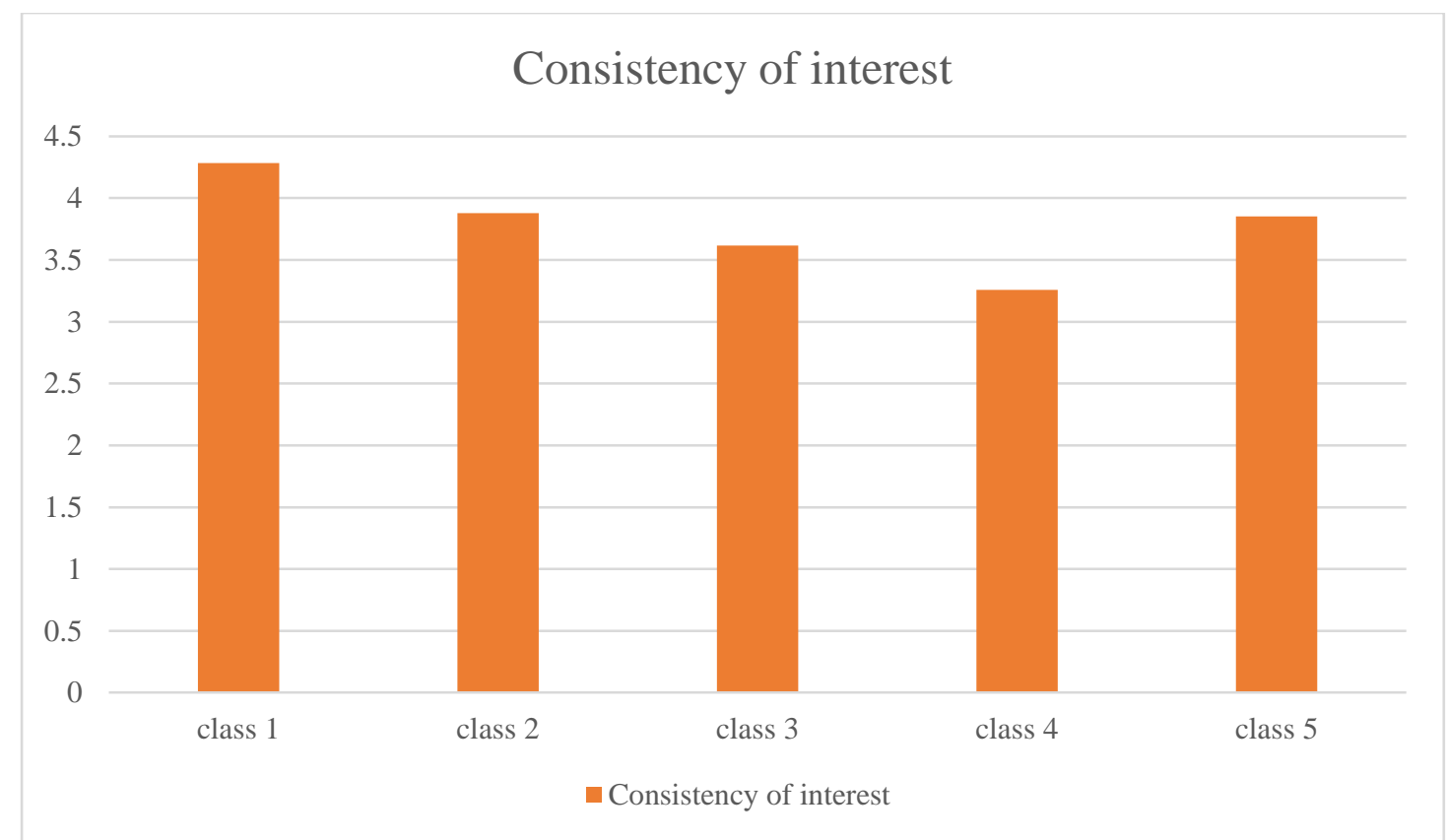

Figure 7. Average values of consistency of interest across the five classes.

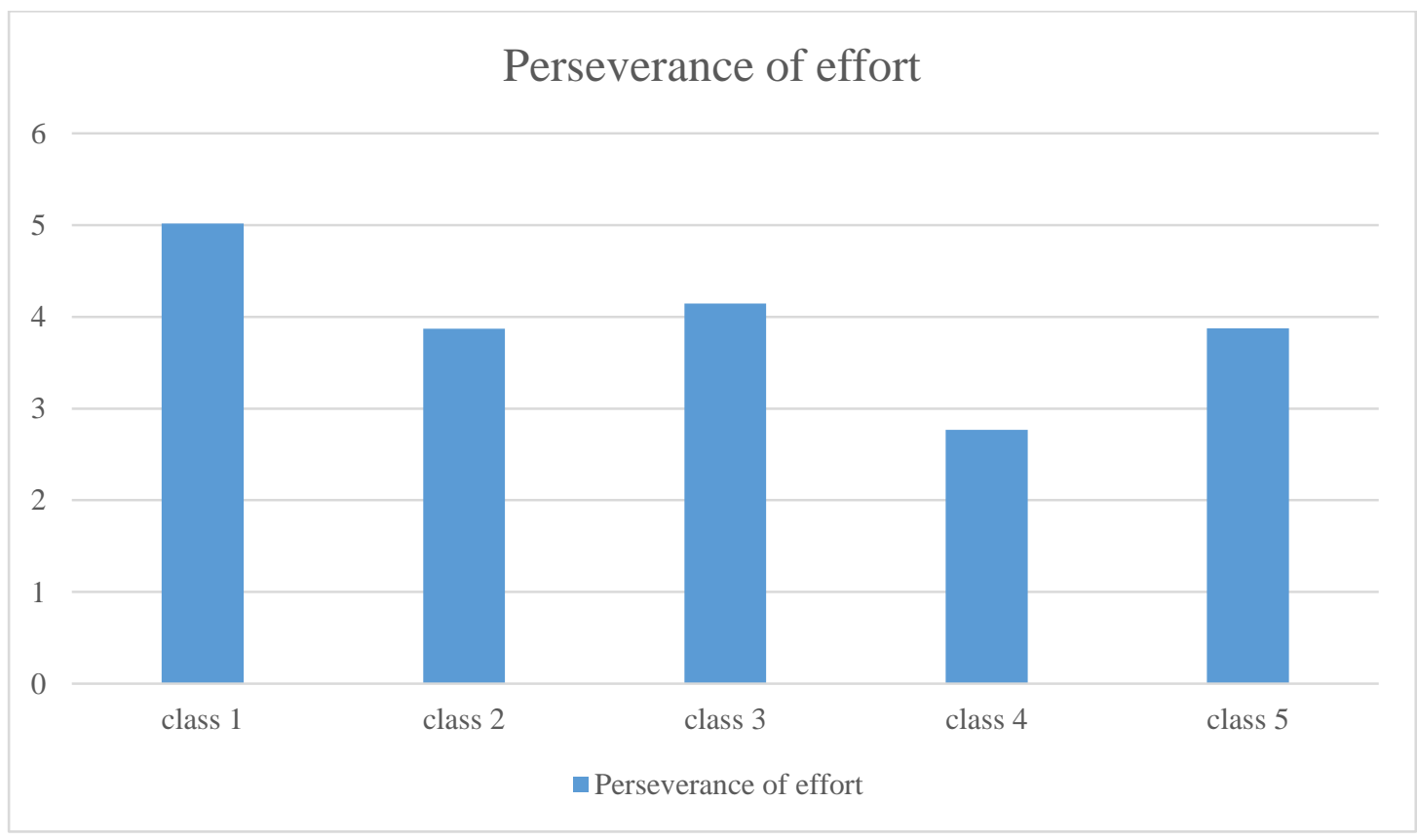

Figure 8. Average values of perseverance of effort across the five classes. 
Incremental theory of intelligence

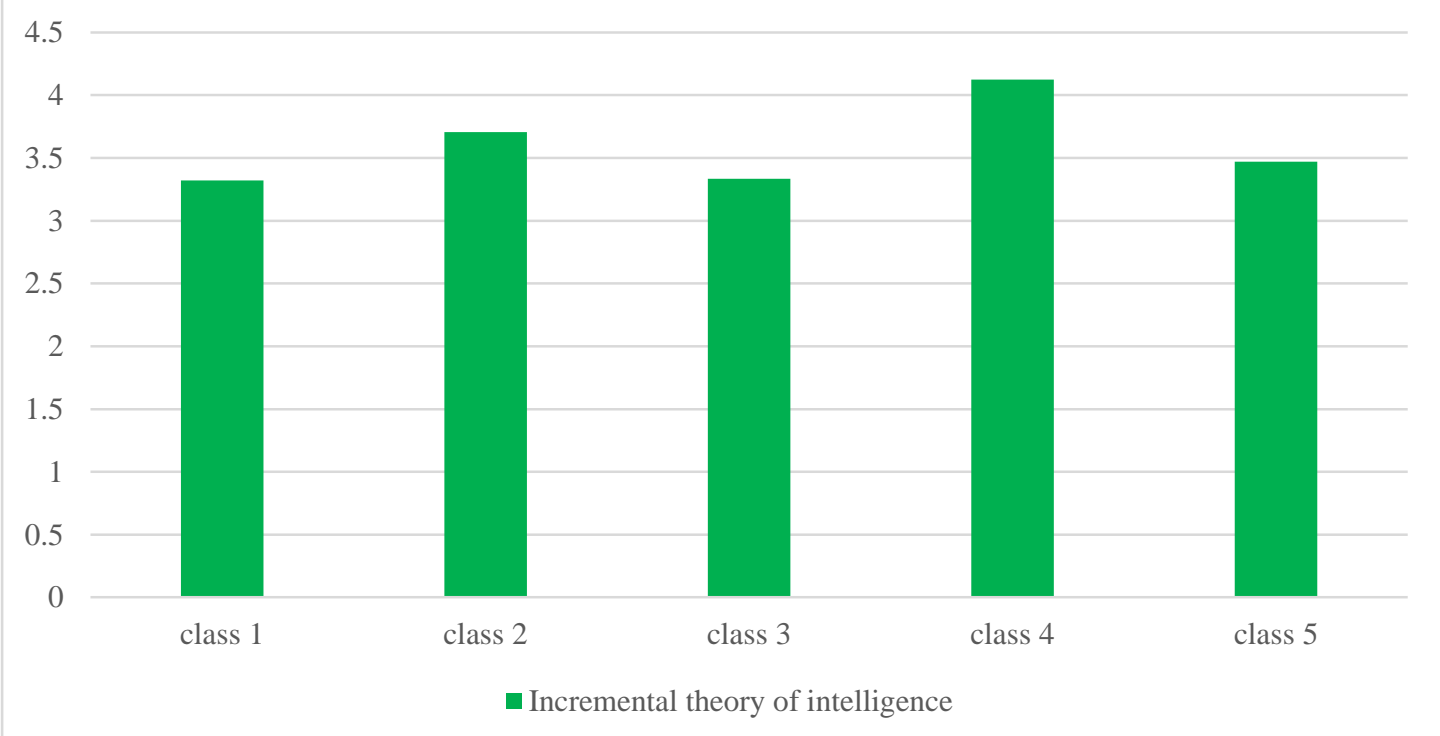

Figure 9. Average values of incremental theory of intelligence across the five classes.

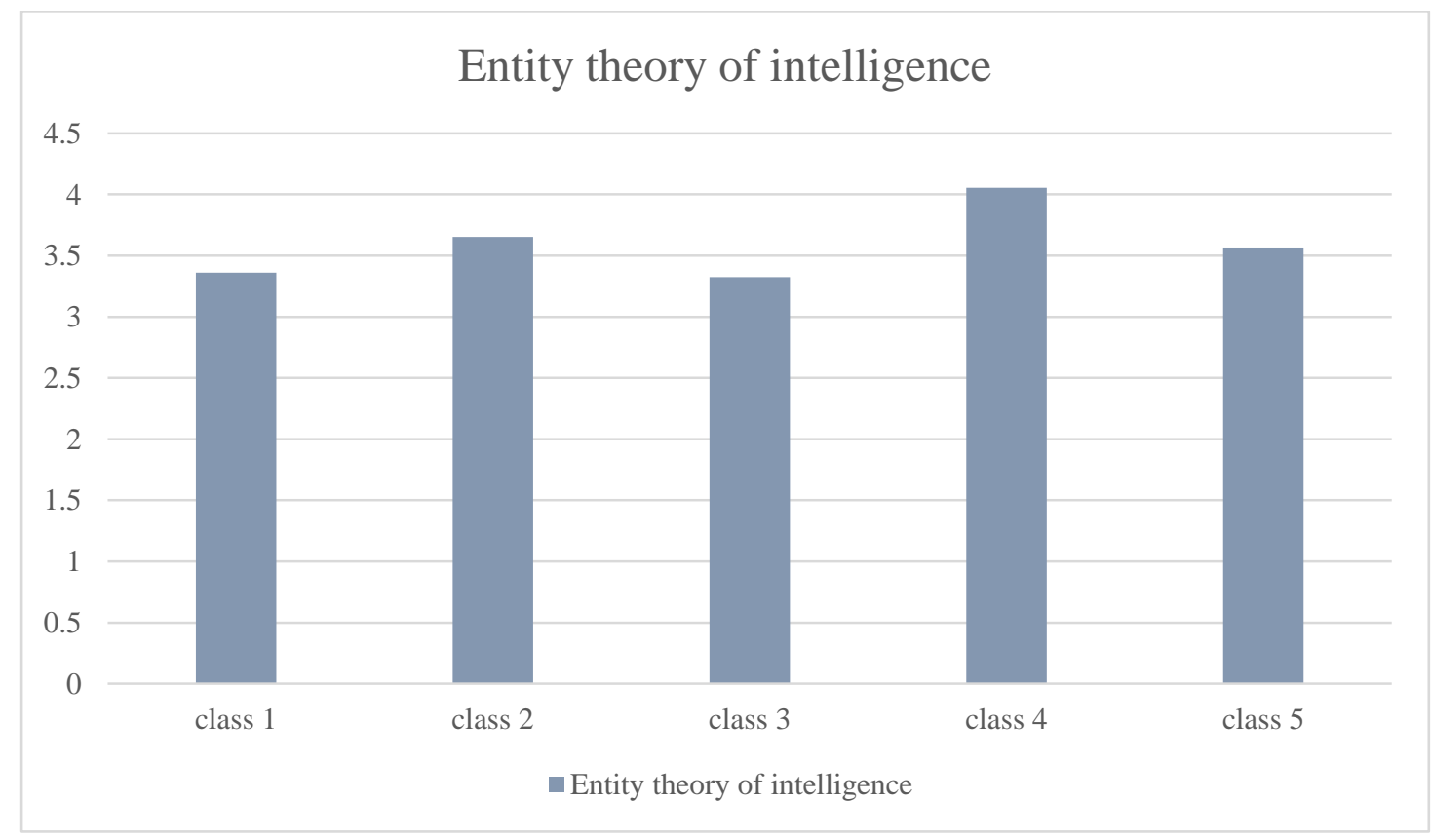

Figure 10. Average values of entity theory of intelligence across the five classes. 


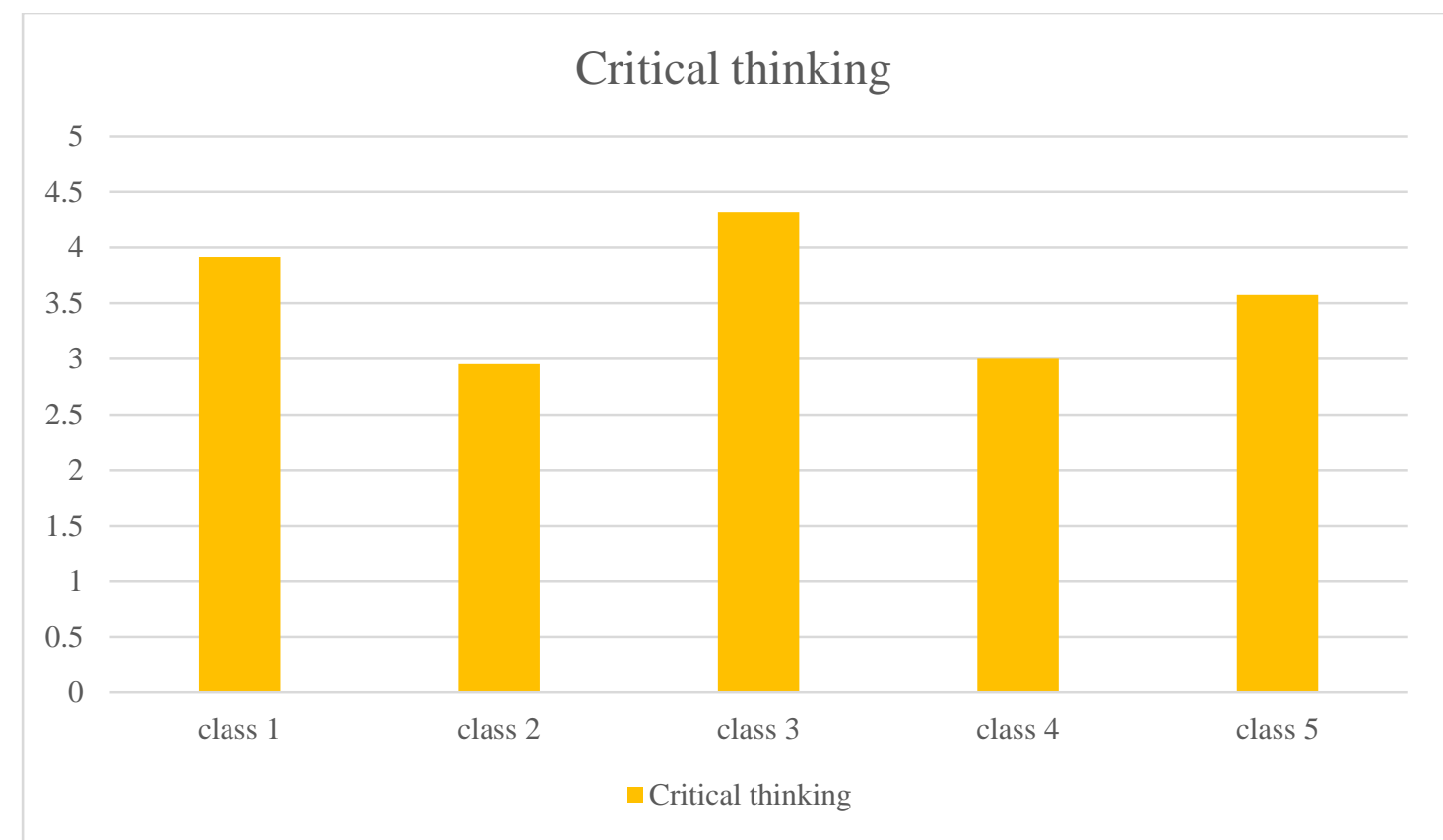

Figure 11. Average values of critical thinking across the five classes.

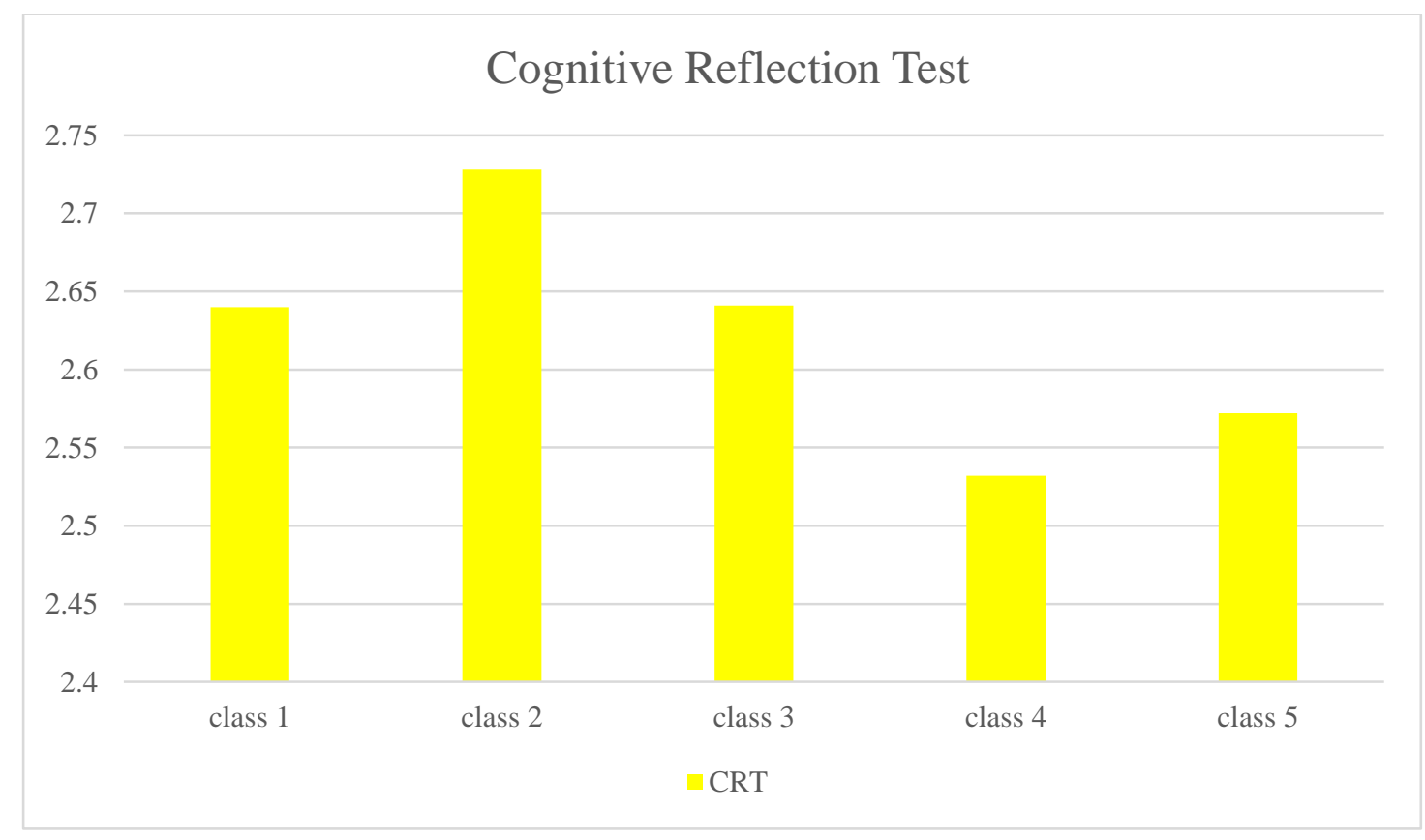

Figure 12. Average values of Cognitive Reflection Test (CRT) scores across the five classes. 


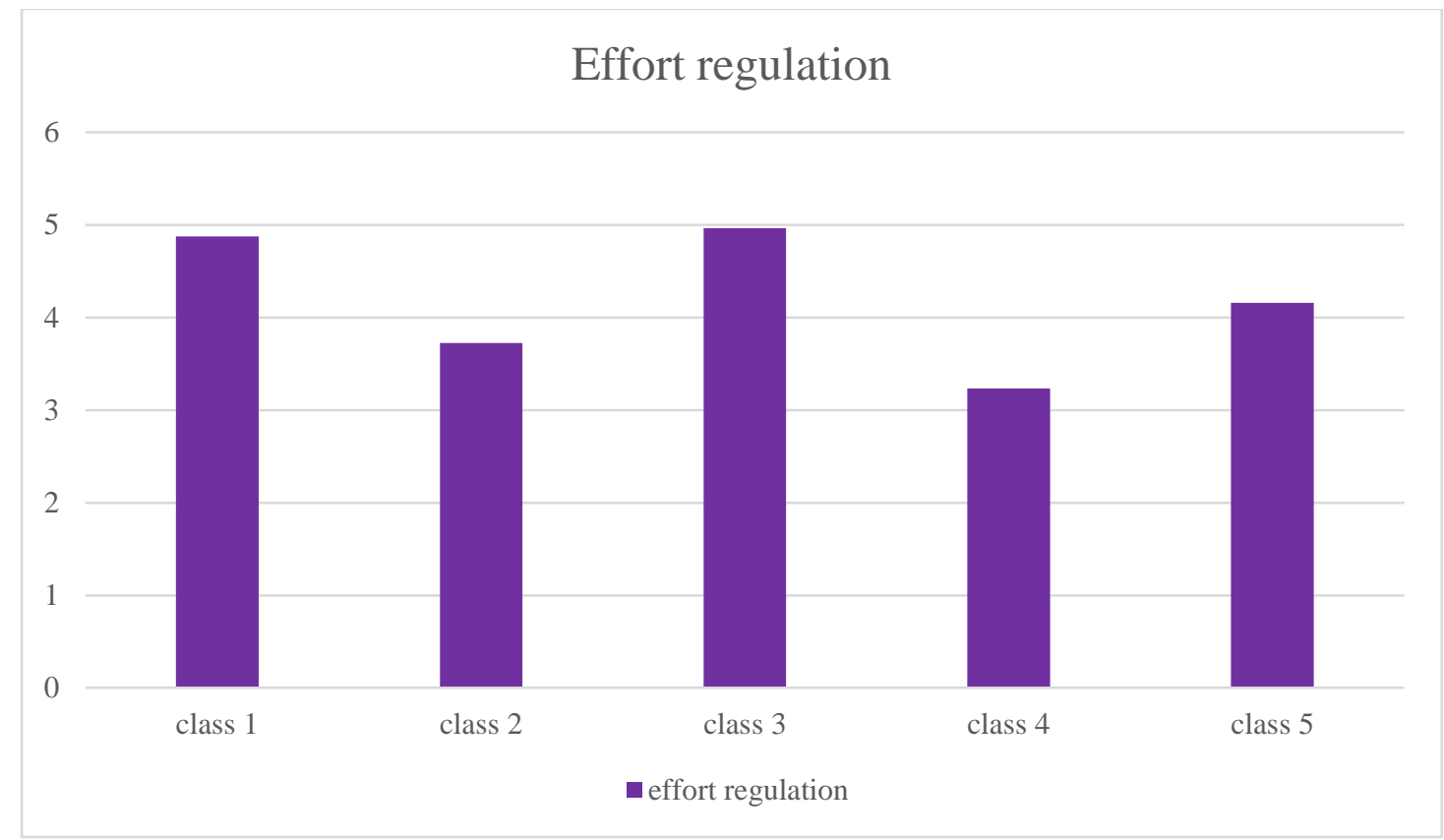

Figure 13. Average values of effort regulation across the five classes.

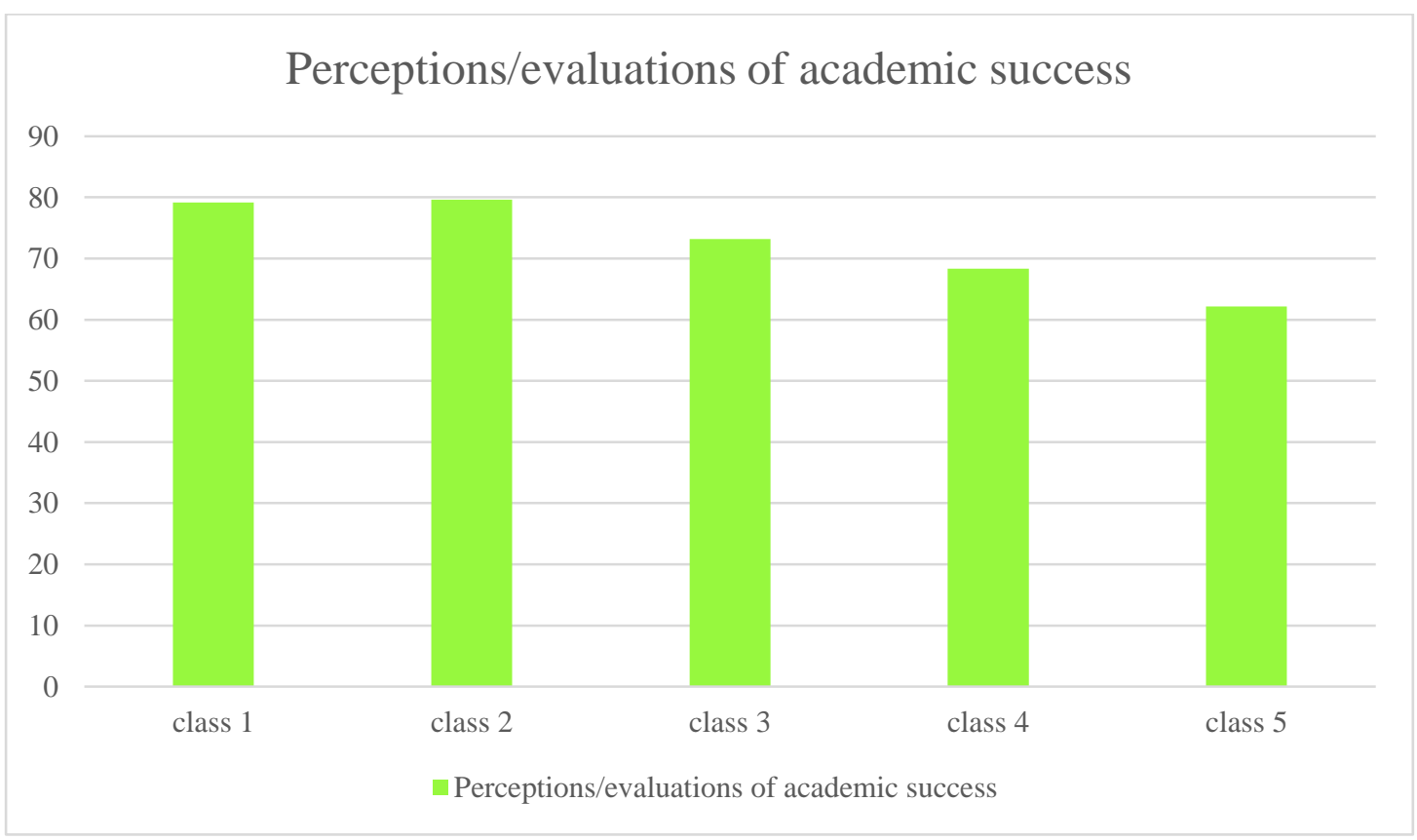

Figure 14. Average values of perceptions/evaluations of academic success across the five classes. 


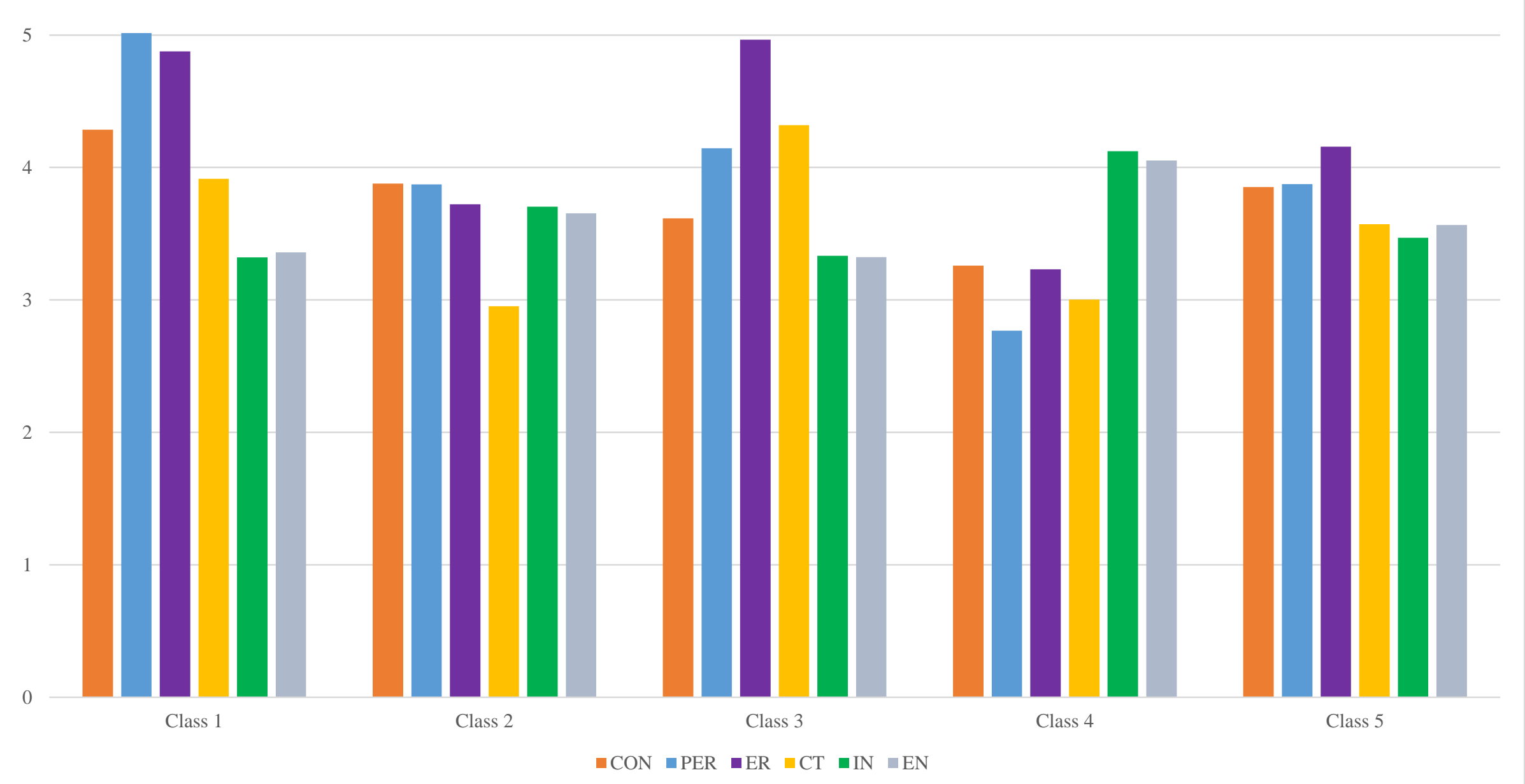

Figure 15. Summary of class-specific mean values of six criteria.

Note. CON = consistency of interest; PER = perseverance of effort; ER = effort regulation; $\mathrm{CT}=$ critical thinking; IN $=$ incremental theory of intelligence; $\mathrm{EN}=$ entity theory of intelligence. 


\section{Summary of Characteristics of the Five Motivation Profiles}

To help characterize each motivation profile as well as highlight similarities and differences with respect to the criteria of interest, Figure 15 summarizes class-specific mean values for six of the eight criteria. CRT is not included in this figure because the tests of significance showed that students classified in the five classes did not differ significantly with regard to this criterion. In addition, perceptions/evaluations of academic success is also not included in this graphic summary of findings because this criterion was recorded on a different scale than all of the other criteria represented in Figure 14 (i.e., 0-100 scale vs. 1-7 scales).

C1 was associated with high/relatively high levels on all but two of the remaining criteria; the exceptions were incremental and entity theories of intelligence, which were weaker than other criteria in this class. Specifically, students classified in $\mathrm{C} 1$ had the highest mean levels of consistency of interest and perseverance of effort of all classes; therefore, $\mathrm{C} 1$ was associated the highest mean level of grit of all classes. For both of these criteria, differences between $\mathrm{C} 1$ and the other classes were statistically significant and moderate to large in magnitude. Notably, students classified in C1 had stronger levels of perseverance of effort than consistency of interest. This class also had the second highest average value of effort regulation of all five profiles. Effort regulation in C1 was not significantly different from effort regulation in $\mathrm{C} 3$, which had the largest mean level of effort regulation, but was significantly stronger than effort regulation in all other classes. In addition, of all classes, students classified in $\mathrm{C} 1$ had the second highest level of critical thinking; differences between $\mathrm{C} 1$ and the other classes were significant and small to moderate in magnitude. Moreover, $\mathrm{C} 1$ was associated with the lowest mean level of incremental theory of intelligence of all classes; nevertheless, there were no significant differences between $\mathrm{C} 1, \mathrm{C} 3$ and $\mathrm{C} 5$ with respect to this criterion. $\mathrm{C} 1$ was also associated with the second lowest level of entity theory of intelligence of all classes; notably, however, only the difference between $\mathrm{C} 1$ and $\mathrm{C} 4$ (which had the largest average value of this criterion) was statistically significant. Importantly, in all classes, including $\mathrm{C} 1$, incremental and entity theories of intelligence were similar in strength. Finally, $\mathrm{C} 1$ was associated with the second highest average score of perceptions of academic success. C1 did not differ significantly from C2, which had the highest mean level of this criterion; also, C1 and C3 were not significantly different with regard to this criterion. However, C1 had a moderately larger average level of self-reported evaluations of academic 
success than $\mathrm{C} 4$ and $\mathrm{C} 5$. To sum up, $\mathrm{C} 1$ was characterized by significantly higher (mean) levels on most criteria (except theories of intelligence) compared to any of the other classes. These findings suggest that students classified in $\mathrm{C} 1$ had generally high levels of consistency of interest, perseverance of effort, effort regulation and critical thinking. Thus, they are likely to be gritty in their goal pursuits and strongly engage in demanding learning tasks/settings. In addition, these students evaluated themselves as being relatively successful in their studies in the university.

C2 was associated with similar mean levels on all but one criteria; the exception was that critical thinking was somewhat weaker than the other criteria in this class. In addition, students classified in C2 exhibited the second highest mean level of consistency of interest and the second lowest average value of perseverance of effort. It should also be noted that, in $\mathrm{C} 2$, perseverance of effort and consistency of interest were very similar in strength. For both of these criteria, only the differences between C2, on the one hand, and C1 (which had the highest mean level of these criteria) and C4 (which had the lowest mean levels of these criteria), on the other hand, were statistically significant. Moreover, C2 was associated with the second lowest mean level of effort regulation; for this criterion, differences between $\mathrm{C} 2$ and the other classes were significant and of moderate to large magnitudes. Moreover, students classified in $\mathrm{C} 2$ had the lowest mean level of critical thinking. With regard to critical thinking, C2 did not differ significantly from $\mathrm{C} 4$, which had the lowest mean level of this criterion. However, the average level of critical thinking in C2 was significantly lower than the average level of critical thinking in the other classes; these differences were small or moderate in magnitude. Furthermore, this class was associated with the second highest mean levels of incremental and entity theories of intelligence. Notably, in C2, both of these criteria were similar in magnitude. For incremental theory of intelligence, only the difference between $\mathrm{C} 2$ and $\mathrm{C} 1$ (which had the lowest average value of this criterion) was statistically significantly. For entity theory of intelligence, C2 did not differ significantly from all other classes.

Finally, students classified in C2 had the highest average value of self-reported evaluations of academic success. C2 was not significantly different from C1 (which had the second largest average value of this criterion). However, differences in mean level of perceptions of academic success between $\mathrm{C} 2$ and the remaining classes were statistically significant and generally medium to large in magnitude. To conclude, $\mathrm{C} 2$ was associated with relatively low levels on perseverance of effort, effort regulation and critical thinking. These findings indicate that students on $\mathrm{C} 2$ are less likely to sustain effort when facing obstacles 
and to strongly engage in demanding or uninteresting learning tasks. Nevertheless, these students evaluated themselves as being relatively successful in their university studies.

For students classified in $\mathrm{C} 3$, on average, perseverance of effort, effort regulation, and critical thinking were stronger than the other criteria. Specifically, students classified in C3 had the second highest level of perseverance of effort of all classes. For this criterion, C3 was not significantly different from C2 and C5, but was moderately lower than C1 and moderately larger than C4. Notably, perseverance of effort was stronger than consistency of interest for $\mathrm{C} 3$. In this class, consistency of interest was, on average, the second lowest among all classes. The average level of consistency of interest was moderately lower in C3 than in $\mathrm{C} 1$ (which had the highest mean levels of this criterion); however, there were no significant differences between $\mathrm{C} 3$ and the other classes with regard to consistency of interest. Moreover, students in C3 had the highest levels of critical thinking and effort regulation of all classes; differences in these criteria between $\mathrm{C} 3$ and the other classes were moderate or large in magnitude. In addition, students classified in C3 exhibited the second lowest mean level of incremental theory of intelligence. C3 was significantly lower than C4 (which had the largest average value of this criterion), but not significantly different from the remaining classes. C3 was also associated with the lowest level of entity theory of intelligence. This criterion was moderately lower in C3 than in C4 (which had the highest mean entity theory of intelligence). However, there were no significant differences among C3 and all other classes with regard to this criterion.

Lastly, C3 was associated with the third highest mean level of perceptions of academic success. However, there were no significant differences with respect to this criterion among $\mathrm{C} 3, \mathrm{C} 1$, and $\mathrm{C} 4$. These findings suggest that students in C3 are likely to persevere in the face of challenges and actively engage in learning tasks that require critical thinking. However, given their moderate levels of consistency of interest, their perseverance of effort may not always be associated with the pursuit of a single goal (e.g., they may sometimes switch goals). Finally, these students had moderate to high evaluations of their own success with regard to studying in the university.

C4 was characterized by relatively low mean levels on all criteria with the exception of incremental and entity theories of intelligence, which were markedly stronger than the other criteria in this class. Specifically, students classified in C4 had the lowest mean levels of consistency of interest and perseverance of effort; thus, they had the lowest mean level of grit of all classes. For consistency of interest, C4 did not differ significantly from C3; C4 had a moderately smaller mean level of this criteria than the remaining classes. For perseverance of 
effort, differences between $\mathrm{C} 4$ and the other classes were significant and of moderate or large magnitudes. Notably, consistency of interest was stronger than perseverance of effort in $\mathrm{C} 4$. This class also had the lowest average value of effort regulation of all classes; with respect to this criterion, differences between $\mathrm{C} 4$ and the other classes were significant and generally large/very large in magnitude. In addition, students classified in C4 had the second lowest level of critical thinking. C4 did not differ significantly from C2 (which had the lowest mean level of this criterion); however, differences in critical thinking between $\mathrm{C} 4$ and the other classes were significant and generally large. Moreover, C4 was associated with the highest mean levels of incremental and entity theories of intelligence. Notably, these two criteria were similar in strength for this class. For incremental theory of intelligence, there was no significant difference between $\mathrm{C} 4$ and $\mathrm{C} 2$, but differences between $\mathrm{C} 4$ and the remaining classes were significant (yet small). For entity theory of intelligence, C4 was moderately larger than $\mathrm{C} 1$ and $\mathrm{C} 3$ (which had the lowest mean level of this criterion), but did not differ significantly from the remaining classes.

Finally, C4 was associated with the second lowest average score of self-reported evaluations of academic success. Nevertheless, only differences between C4 (on the one hand), and C1 and C2 (on the other hand), which had the largest average scores of this criterion among all classes, were statistically significant. The findings suggest that students classified in C4 may be at risk of amotivation and disengagement at school. This is the case because $\mathrm{C} 4$ was associated with significantly lower (mean) levels on most criteria than any of the other classes.

C5 was associated with similar mean levels of all criteria; effort regulation was the strongest criterion in this class. As it was the case in C2, for C5, consistency of interest and perseverance of effort were almost identical in strength. Across classes, students classified in C5 had the third highest mean levels of both consistency of interest and perseverance of effort. For both of these criteria, only differences between C5 (on the one hand) and C1 and C4 (on the other hand) were significant (medium to large effect sizes). This class also had the third highest average value of effort regulation. Difference between C5 and the other classes with regard to this criterion were significant and generally large. Similarly, students in C5 exhibited the third highest level of critical thinking. For this criterion, differences between C5 and the other classes were significant and small or moderate in magnitudes. In addition, C5 was associated with the third highest (and similar) mean levels of incremental and entity theories of intelligence. Incremental theory of intelligence was, on average, significantly smaller in C5 than in C4 (which had the highest mean level of this criterion); however, levels 
of incremental theory of intelligence did not differ significantly in C5, C1, C2, and C3. For entity theory of intelligence, differences between C5 and all the other four classes were not statistically significant.

Finally, students classified in C5 had the lowest average score of self-reported evaluations of academic success. There was no significant difference in this criterion between C5 and C4. However, differences between C5 and the remaining classes were significant and of large magnitudes.

In summary, C5 was characterized by having slightly above/below average levels on all criteria except perceptions of academic success. Considering that they had moderate levels of consistency of interest and perseverance of effort, these students are less likely to sustain their interest and effort for long-term goals than their counterparts in C1. Moreover, their moderate levels of effort regulation and critical thinking suggest that they are not very strongly engaged in uninteresting learning tasks or use consistently critical thinking in their learning. Finally, these students had the lowest evaluations of their success with studies at university of all five classes.

\section{Chapter Summary}

In this chapter, I reported the quantitative findings from the first phase of the research. Five distinct motivation profiles that mapped the interrelationships among the key motivation orientations were uncovered for the sample of Chinese undergraduate students in the research. Subsequent analyses showed that there were important differences among these profiles with regard to most of the motivation effects (i.e., grit, theories of intelligence, critical thinking, effort regulation, and perceptions/evaluations of academic success). In the next chapter I will report the qualitative findings in the second phase of the research. 


\section{Chapter Five Qualitative Findings}

This chapter focuses on the analysis of qualitative data collected from the samples of students who participated in the qualitative phase of the study. Each of the five multiple case studies was specifically associated with a specific motivational profile. Findings for each case, which are presented separately, focus on how the approaches to learning or learning-related tasks/activities reported by the interview participants mapped onto the characteristics of their motivation profile.

\section{Case 1: Participants with the C1 Profile}

Three themes emerged from the qualitative data provided by the four interviewees who had the $\mathrm{C} 1$ profile (i.e., Lemon, Lily, Southerly, and Wang). These themes are: "alignment with strong assessment", "alignment with strong locomotion", and "alignment with dual strong promotion and prevention".

Alignment with strong assessment. The approaches to learning and learning-related tasks/activities reported by the interviewees recruited from this profile align with their strong assessment orientations. Specifically, they strived for truth effectiveness, made critical comparisons and evaluations, and weighed up options in the decision-making processes.

Striving for truth effectiveness. All participants stated that they tried to find and comprehend what was real or correct about themselves and their experiences. For instance, Lemon shared her experience when she received negative feedback from the foreign teacher in a writing course.

I spent a lot of time thinking about why the teacher gave me such comments and what was wrong with the writing techniques I have been taught at high school. (Lemon)

Similarly, when recalling his experiences of failure, Wang noted that he always reflected on what he had done and what flaws he had, so that he could have a clearer direction where to focus his efforts in the future.

Making critical comparisons and evaluations. When learning theories in their courses, all participants compared and evaluated what they learned and attempted to attain a clear understanding of the particular knowledge. Southerly cited her learning experience as an example.

I would think about the similar theories I had learned before. I would read 
the learning materials and compare what the similarities and differences there were. (Southerly)

Wang also tried to compare different assertions and find out what caused the differences, so that he could achieve a comprehensive picture about a particular topic.

Apart from evaluating the knowledge she learned in her courses, Lily stated that she tended to compare herself to her classmates and that this evaluation caused her some concerns.

Sometimes you would feel anxious if you saw the others make more progress. 'Oh, she finished this book, but I haven't begun to read it." In this case, you would feel worried. (Lily)

This self-evaluative concern and the attendant emotional reaction are also consistent with a strong assessment orientation.

Weighing up options. Two students reported that they weighed up alternatives before making important decisions. For instance, Lemon recalled her hesitation over the choice between her current major and English for a postgraduate programme. She wanted to switch to the major in English, for it would provide her more opportunities for future development. However, she was afraid that she did not have much edge compared to students who majored in English.

So I have weighed up the benefits and the costs, but it's very hard to make the final decision. (Lemon)

Wang had a similar experience when he applied for a postgraduate program.

I hesitated about whether I should choose a top university or an ordinary one that could secure me a placement. I had been weighing up the pros and cons of the options for a long time before I made the final decision. (Wang)

These accounts suggest that, in order to make the best choices, the students considered extensively which goals they should pursue by weighing up different options. This resulted in indecisiveness. Taken together, these experiences reflect a strong assessment orientation.

Alignment with strong locomotion. The approaches to learning or learning-related tasks/activities reported by all four participants align with their strong locomotion orientations. To initiate and maintain smooth movement, they preferred to do things step by step, were keen to move on to the pursuit of new goals after achieving a goal, tended to give up original goals when experiencing little or no progress, and they started working at once when they received a task.

Doing things step by step. Three participants reported that they preferred to do their 
work step by step "in an organized way" (Lily). Lemon discussed some of the learning strategies she found useful to ensure continuous progress.

I assigned myself some easy learning tasks, and became engaged in learning, and then gradually increased the level of task difficulty, step by step, to achieve what I wanted to complete.... More importantly, I have to make practical plans and make continuous progress step by step according these plans. (Lemon)

Southerly also related her experience in a course she found interesting. In this class, she enjoyed doing exercises according to the step-by step instructions.

I felt great about moving smoothly from one task to another till completion. (Southerly)

By doing things step by step, these students maintained smooth and steady progress in learning, which is consistent with having a strong locomotion orientation.

Moving to the next goals. Three participants reported that they preferred to move on to pursue new goals immediately after they had achieved a goal. In Southerly's words, "Life is seeking for and realizing something."

Wang preferred planning and worked for the next goals once he attained one goal in his schedule. Lily also described a similar way of setting goals and working towards them.

After each exam, I would set the goal about what grade I would get in the next exam. Only in this way can I encourage myself to move forward. (Lily)

These reports are consistent with theorizing and findings that indicate that individuals who have a strong locomotion orientation do not like to be inactive. They desire to move, to change, and to sustain movement and change (Kruglanski et al., 2000).

Giving up original goals when experiencing no progress. Two students reported that they tended to abandon their goals when they saw no progress. Southerly stated that she might give up her pursuit of a postgraduate program if she failed to get an offer this year to enter a postgraduate program.

I am afraid I will fail again and waste a lot of time. I will try something else. (Southerly)

In addition, Wang shared his similar experience in preparing for the postgraduate exam.

I felt like that I was stuck in a bottleneck period, feeling no more progress in learning and having mood swings. And then I went to the job fairs on campus. (Wang)

These reported experiences are consistent with theorizing showing that high 
locomotors are likely to abandon original goals which require them to overcome obstacles and when they experience little or no progress (Kruglanski et al., 2000).

Working on an assignment immediately. All participants reported that they preferred to start to work on an assignment as soon as possible rather than procrastinate. For example, when she was asked to write an essay, Lemon started to collect related materials immediately. Similarly, Wang tended to work on his assignments right away.

The assignment always caused a sense of urgency in my mind. I wanted to complete it as soon as possible. (Wang)

The motivation to initiate action reported by these participants is consistent with a strong locomotion orientation.

Alignment with dual strong promotion and prevention. The interview data from the interviewees recruited from Class 1 align with the quantitative findings that these participants had both strong promotion and prevention orientations. Specially, they strived for ideals (i.e., hopes and aspirations), were motivated by success, positive feedback and positive role models and were enthusiastic in their learning. At the same time, they made efforts to fulfill their duties and responsibilities, felt motivated by failure and negative feedback, and were careful with studies at university.

Striving for ideals and working to fulfil responsibilities. All participants from Class 1 reported promotion-related reasons that motivated them to study at university. In particular, they were motivated to achieve their own aspirations and hopes. For example, Lemon was working hard to prepare for the postgraduate entrance exam. She stated,

I have been motivated by my aspirations for my dream university and my beautiful future. (Lemon)

Lily also reported that her aspiration of being a graduate student in her dream university had driven her in the "hard life" of preparing for the postgraduate entrance exam. She observed,

I have a lot of beautiful hopes for studying and life. There is a difference between the reality and my aspirations. This has motivated me to study hard. (Lily)

At the same time, they noted that they were motivated by their obligations to their families. Lily confided,

...I have to work hard in order to offer them (i.e., my parents) a better life, which also motivates me to study hard. (Lily)

In addition, two participants viewed their parents' expectations as their responsibilities. Lily shared her learning experiences during her early school life. 
...I had to meet their expectations that I could go to the best schools and a top university. I had worked hard to be the trophy kid in all parents' eyes, admitted to the key classes in the top schools. (Lily)

Apart from the obligations to her family, Southerly also reported other responsibilities that she thought she had to uphold.

I am a college student and my duty is to study hard. I study to meet the university requirements for graduation instead of failing courses.

(Southerly)

Moreover, all four participants' strong prevention orientations were primed in certain contexts (e.g., final exams). They reported setting prevention-related goals, such as "not to fail in these exams" (Lily) or having to "make sure that I learn every course well" (Lemon). Wang confided,

...if you fail in the exam, you are likely not be able to graduate. You have to work hard to prevent these serious consequences. (Wang)

Wang's answer suggests that he had a strong prevention orientation in this context and worked hard to avoid failure.

The participants classified in Class 1 worked towards their aspirations, which characterizes promotion-related motivation. Meanwhile, they also worked hard to meet their responsibilities and achieve their prevention goals. These accounts reflect dual strong promotion and prevention orientations.

Being motivated by success and/or failure. These participants reported different responses to success/failure in learning. The emotional experiences regarding success and failure, which were reported by two interviewees, reflect a strong promotion orientation.

Success... gives me a sense of achievement and confidence to work harder. But failure is a blow to me, so that I might quit. (Southerly)

These accounts suggest that success motivated her to devote more time and effort to their studies. However, the negative emotions she experienced after the failure demotivated her.

In contrast, Wang reported that failure, more than success, motivated him to make effort:

To fail once allows me to calm down and reflect on my own flaws, so that I have a clearer direction and am motivated to go on working.

Success...tends to obscure the need for improvement. (Wang) Thus, although he had a dual promotion and prevention focus, he probably had stronger prevention than promotion. As a result, failure prompted him to pay particular attention to failure-related information, which might help him avoid losses in the future. Therefore, 
failure appeared to increase his vigilance.

Finally, Lily stated that she was motivated by both success and failure. She observed, ...after this success, I thought that the goal wasn't challenging enough and thus I wanted to try to achieve a more ambitious goal. (Lily)

Then, she commented on her response to failure,

... failure would make me feel tense, kind of under pressure, so that I would try harder to prove I was capable. I would be highly motivated by failure and more determined to make it next time. (Lily)

The data provided by this student are consistent with having both strong promotion and prevention orientations. Her promotion focus contributed to happiness after success, which made her keener for more success. In addition, her prevention focus led to anxieties after failure, which enabled her to be more cautious. As a result, she was not complacent after success and was resilient after failure.

In line with their strong promotion orientations, these participants were motivated by success to work harder. Consistent with their strong prevention orientations, they were motivated by failure to devote more efforts. Importantly, being motivated by both success and failure is consistent with the dual strong promotion and prevention orientations that characterized the $\mathrm{C} 1$ profile.

Being motivated by positive feedback/role models and by negative feedback. These students reported that positive feedback and positive role models had a motivating effect upon them. In particular, all four students observed that they were motivated by positive feedback.

...positive comments like praise will make me feel happier and more motivated. (Lemon)

Positive feedback led to strong positive emotions such as happiness, which helped to strengthen their motivation to succeed.

Moreover, Lemon commented on the important role that the positive role models played in her persistence in coping with the difficulties in her Literature course.

... those who have a deep literary knowledge are charming, and their manners and writings impress me. ...So I just want to be that type of person. (Lemon)

In addition, she said she was also motivated by the successful experiences of those seniors who had been admitted to the postgraduate programs. Encounters with these positive role models enabled her to identify the achievements that she desired and the strategies she 
needed to use to be successful. These positive role models strengthened her motivation and persistence in the learning tasks at hand.

Meanwhile, these students all reported that they were also motivated by negative feedback. Lily commented,

I will feel a bit upset when I receive negative comments on my work, but I will also try to find out my weakness and work harder. (Lily)

Consistent with their strong promotion orientations, they increased their enthusiasm when they received positive feedback and saw positive role-models. At the same time, consistent with their strong prevention orientations, they were sensitive to negative feedback, which provided information about the mistakes and the strategies that they needed to prevent failure in the future.

Being enthusiastic and cautious. All four participants reported their enthusiasm to achieve their aspirations. For example, when they met difficulties in their courses, they readily sought help from their teachers and peers.

Whenever I had questions in any course, I would ask. I would turn to the teacher and my classmates for help. (Southerly)

Wang had a group of "buddies" who also worked on the postgraduate entrance exam.

When I was down, I talked with my buddies and then l felt relieved. We encouraged each other and urged each other. (Wang)

These examples are typical of using eager strategies in learning settings (Molden \& Rosenzweig, 2016).

Moreover, they were open to change and preferred to have different experiences in life. They chose to apply for some top universities in cosmopolitan cities in China to pursue their postgraduate studies to gain more opportunities of academic development. In addition, when facing obstacles in learning, these students had an optimistic attitude.

I am not overwhelmed by difficulties in learning. I am positive about my future and believe that I will finally succeed. (Southerly)

At the same time, all four participants noted using strategies to guard against failure and to not make mistakes. For instance, in order not to fail in the final exam, they followed the course requirements carefully and "reviewed the key points the teacher provided for the final exam" (Southerly). Southerly also reported that she did not expend effort on the questions that would not be tested in the exams.

Moreover, these participants stated that they checked their work repeatedly.

Especially in exams... I looked through the papers again and again, 
revisiting the test questions I thought I might do wrong, to make sure that I did not commit any error. (Southerly)

In addition, they preferred being accurate. When he wrote an essay, Wang checked what he wrote carefully. To avoid plagiarism, he located the parts with a high similarity percentage to existing sources and revised these parts. He commented,

It's carelessness that makes your grades unsatisfactory. (Wang)

Being enthusiastic helped these participants' advancement. Meanwhile, being careful helped them prevent mistakes and negative outcomes. This outlook is consistent with their dual and strong promotion and prevention orientations.

A summary of their interview data is presented in Table 36. The quantitative results revealed that the motivation profile underlying Class 1 was characterized by having a combination of high/very high average values on all four motivation orientations, with prevention being the strongest of the four motivation orientations. As I discussed previously, the interview data from the interviewees recruited from Class 1 suggested that their reported approaches to learning and learning-related tasks/activities aligned well with the characteristics of this motivation profile. 
Table 36

Summary of the Four Participants' Data for Each Theme (C1)

\begin{tabular}{|c|c|c|c|c|c|}
\hline \multirow[t]{2}{*}{ Theme } & \multirow[t]{2}{*}{ Category } & \multicolumn{4}{|c|}{ Participant } \\
\hline & & Lemon & Lily & Southerly & Wang \\
\hline \multirow[t]{3}{*}{$\begin{array}{l}\text { Alignment } \\
\text { with strong } \\
\text { assessment }\end{array}$} & Striving for truth effectiveness & $\begin{array}{l}\text { Reflected on the comments } \\
\text { and her mistakes in writing }\end{array}$ & $\begin{array}{l}\text { Reflected on her failure } \\
\text { experiences }\end{array}$ & $\begin{array}{l}\text { Reflected on her learning } \\
\text { experiences and } \\
\text { identified the ways to } \\
\text { overcome the difficulties }\end{array}$ & $\begin{array}{l}\text { Reflected on his failure } \\
\text { experiences }\end{array}$ \\
\hline & $\begin{array}{l}\text { Making critical comparisons } \\
\text { and evaluations }\end{array}$ & $\begin{array}{l}\text { Compared and evaluated } \\
\text { supporting evidence }\end{array}$ & $\begin{array}{l}\text { Compared herself to the } \\
\text { others }\end{array}$ & $\begin{array}{l}\text { Compared similar } \\
\text { theories }\end{array}$ & $\begin{array}{l}\text { Compared different } \\
\text { assertions }\end{array}$ \\
\hline & Weighing up options & $\begin{array}{l}\text { Weighed up alternative } \\
\text { majors for a postgraduate } \\
\text { programme }\end{array}$ & & & $\begin{array}{l}\text { Hesitated about choosing } \\
\text { universities }\end{array}$ \\
\hline \multirow{4}{*}{$\begin{array}{l}\text { Alignment } \\
\text { with strong } \\
\text { locomotion }\end{array}$} & Doing things step by step & Made progress step by step & $\begin{array}{l}\text { Did work in an organized } \\
\text { way }\end{array}$ & $\begin{array}{l}\text { Followed step-to-step } \\
\text { instructions }\end{array}$ & $\begin{array}{l}\text { Did work in an organized } \\
\text { way }\end{array}$ \\
\hline & Moving to the next goals & $\begin{array}{l}\text { Worked toward new goals } \\
\text { at once }\end{array}$ & $\begin{array}{l}\text { Moved on to new goals at } \\
\text { once }\end{array}$ & $\begin{array}{l}\text { Pursued new goals } \\
\text { immediately after } \\
\text { achieving the previous } \\
\text { goals }\end{array}$ & $\begin{array}{l}\text { Worked for new goals at } \\
\text { once }\end{array}$ \\
\hline & $\begin{array}{l}\text { Giving up original goals when } \\
\text { experiencing no progress }\end{array}$ & & & $\begin{array}{l}\text { Worked for other goals if } \\
\text { she failed in the } \\
\text { postgraduate entrance } \\
\text { exam }\end{array}$ & $\begin{array}{l}\text { Looked for new goals } \\
\text { when perceiving no } \\
\text { progress }\end{array}$ \\
\hline & $\begin{array}{l}\text { Working on an assignment } \\
\text { immediately }\end{array}$ & $\begin{array}{l}\text { Worked on her essays as } \\
\text { soon as possible }\end{array}$ & $\begin{array}{l}\text { Worked on her } \\
\text { assignments immediately }\end{array}$ & $\begin{array}{l}\text { Worked on her } \\
\text { assignments immediately }\end{array}$ & $\begin{array}{l}\text { Worked on his } \\
\text { assignments as soon as } \\
\text { possible }\end{array}$ \\
\hline
\end{tabular}




\begin{tabular}{|c|c|c|c|c|c|}
\hline \multirow[t]{2}{*}{ Theme } & \multirow[t]{2}{*}{ Category } & \multicolumn{4}{|c|}{ Participant } \\
\hline & & Lemon & Lily & Southerly & Wang \\
\hline \multirow[t]{4}{*}{$\begin{array}{l}\text { Alignment } \\
\text { with dual and } \\
\text { strong } \\
\text { promotion } \\
\text { and } \\
\text { prevention }\end{array}$} & $\begin{array}{l}\text { Striving for ideals and working } \\
\text { to fulfil responsibilities }\end{array}$ & $\begin{array}{l}\text { Studied to achieve } \\
\text { aspirations; } \\
\text { Lived up to parental } \\
\text { expectations; } \\
\text { Had prevention-related } \\
\text { goals }\end{array}$ & $\begin{array}{l}\text { Studied to achieve } \\
\text { aspirations; } \\
\text { Studied to improve } \\
\text { herself; } \\
\text { Fulfilled obligations to } \\
\text { family; } \\
\text { Met parental } \\
\text { expectations; } \\
\text { Set prevention-related } \\
\text { goals }\end{array}$ & $\begin{array}{l}\text { Studied to achieve } \\
\text { aspirations; } \\
\text { Fulfilled obligations to } \\
\text { family; } \\
\text { Fulfilled duties as a } \\
\text { student; } \\
\text { Had prevention-related } \\
\text { goals }\end{array}$ & $\begin{array}{l}\text { Studied to achieve } \\
\text { aspirations; } \\
\text { Fulfilled obligations to } \\
\text { family; } \\
\text { Lived up to parental } \\
\text { expectations; } \\
\text { Had prevention-related } \\
\text { goals }\end{array}$ \\
\hline & $\begin{array}{l}\text { Being motivated by success } \\
\text { and/or failure }\end{array}$ & Felt motivated by success & $\begin{array}{l}\text { Felt motivated by success } \\
\text { and failure }\end{array}$ & Felt motivated by success & Felt motivated by failure \\
\hline & $\begin{array}{l}\text { Being motivated by positive } \\
\text { feedback and positive role } \\
\text { models and by negative } \\
\text { feedback }\end{array}$ & $\begin{array}{l}\text { Felt motivated by positive } \\
\text { and negative feedback, and } \\
\text { positive role models }\end{array}$ & $\begin{array}{l}\text { Felt motivated by } \\
\text { positive and negative } \\
\text { feedback }\end{array}$ & & $\begin{array}{l}\text { Felt motivated by positive } \\
\text { and negative feedback }\end{array}$ \\
\hline & Being enthusiastic and cautious & $\begin{array}{l}\text { Looked for more } \\
\text { opportunities; } \\
\text { Followed the course } \\
\text { requirements; } \\
\text { Checked her assignments }\end{array}$ & $\begin{array}{l}\text { Asked classmates for } \\
\text { help; } \\
\text { Wanted to have different } \\
\text { experiences; } \\
\text { Had an optimistic } \\
\text { outlook; } \\
\text { Checked her work }\end{array}$ & $\begin{array}{l}\text { Asked teachers and } \\
\text { classmates for help; } \\
\text { Wanted to have different } \\
\text { experiences; } \\
\text { Had an optimistic } \\
\text { outlook; } \\
\text { Did not review the } \\
\text { questions that were not } \\
\text { tested in exams; } \\
\text { Had a thorough check for } \\
\text { mistakes }\end{array}$ & $\begin{array}{l}\text { Asked teachers and } \\
\text { classmates for help; } \\
\text { Wanted different } \\
\text { experiences; } \\
\text { Was optimistic; } \\
\text { Checked to avoid } \\
\text { plagiarism }\end{array}$ \\
\hline
\end{tabular}




\section{Case 2: Participants with the C2 Profile}

Four themes emerged from the qualitative data provided by the four participants who had the C2 profile (i.e., Jasmine, Linnaeus, Qian, and Sharon). These themes are "alignment with below average assessment", "alignment with below average locomotion", "alignment with below average promotion", and "alignment with strong prevention".

Alignment with below average assessment. The approaches to learning and learningrelated tasks/activities reported by the participants in this class align with their below average levels of assessment. Specifically, they tended to compare themselves to the others, attempted to be accurate, weighed up options, and reflected on their previous experiences. Notably, they did not engage in critical evaluation of what they learned in their courses.

Making critical comparisons and evaluations. Two participants reported that they tended to compare themselves to their classmates and this comparison caused them some concerns. As Linnaeus confided,

I always compare myself to everyone around me in terms of everything. For instance, sometimes I felt depressed when I heard that one of my friends achieved something and I kept thinking about it. These comparisons have a negative effect on me. I suffer from negative emotions and make me feel less self-confident. (Linnaeus)

These students tended to compare themselves to standards as well as to people around them. In line with the tenets of the regulatory mode theory (Kruglanski et al., 2000), these comparisons, which characterize a strong assessment orientation, made them vulnerable to negative emotions and low self-confidence.

However, these participants reported that they did not evaluate critically the particular knowledge they learned in their courses.

Most of the time, I didn't reflect on what I had learned, and I thought it's ok to accept what the teachers taught.... I just listened, took notes, and then memorized it. (Sharon)

These accounts are consistent with a weak assessment orientation.

Striving for accuracy. Three participants stated that they tried to be accurate and checked their work. Qian recalled that she checked her paper repeatedly to ensure that the format must be exactly the same as the required template. In addition, when she prepared her slides for a presentation in class, she had to make sure every slide was what she wanted to be. 
Another example of striving for accuracy in learning was reported by Linnaeus. ...The teacher mentioned some foreign literary works in class. But I couldn't remember or spell the names of the works or the writer. I would go to search for the accurate information. ...finding the accurate information would make me reassured. (Linnaeus)

By trying to be accurate, these participants ensured certainty and avoided ambiguity in learning. These tendencies reflect a strong assessment orientation.

Weighing up options. Two participants reported that they weighed up options before they made the final decisions. In deciding which university to apply for a postgraduate programme, Linnaeus compared thoroughly entrance exam papers of universities in north and south China.

I have to weigh up the pros and cons for each alternative university before I make the final choice. (Linnaeus)

Weighing up alternatives helped them identify the best option. This tendency to spend a lot of time investigating the benefits and costs of all options reflects a strong assessment orientation and could result in indecisiveness.

Reflecting on previous personal experiences. Two students reported that they felt regretful about their previous behaviors. For example, when talking about her failure to be admitted into the popular majors after the college entrance exam, Qian commented,

If I had known in advance that my score was not high enough for those popular majors, I might have applied for the major of the human resources, or I could have chosen the major of administrative management. (Qian)

Assessment involves making critical evaluations of one's decisions, which includes engaging in counterfactual thinking, with the intention to do the right thing in future. Reflecting on what would have happened if they only had taken different actions, these participants tended to feel remorse for their previous behaviors.

Taken together, these participants reported tendencies related to both strong and weak assessment orientations.

Alignment with below average locomotion. The qualitative data reported by the participants from this class align with their below average locomotion orientations. On the one hand, these participants shared some tendencies associated with a strong locomotion orientation. Specifically, to maintain smooth flow of work, they preferred to do things step by step. On the other hand, they were not keen to move on to the next 
goals, and not focused on tasks due to distractions. Both of these tendencies are associated with a weak locomotion orientation.

Doing things step by step. Two participants reported that they preferred to "do the work step by step" (Sharon). In particular, Jasmine said that she enjoyed doing her accounting assignments such as entry writing.

I felt great doing step by step. I analyzed its nature, wrote it down, and worked out the amounts of money. I had to do a lot of calculations to work out that final number. (Jasmine)

By doing her exercises sequentially, she maintained steady progress from one entry to another, which is consistent with having a strong locomotion orientation.

Moving to the next goal. Two participants noted that they preferred to take a break before moving on to pursue new goals. When asked what she would do once she achieved one goal, Jasmine confided,

After taking the exam for the teacher's certificate, I took some time off before working on the English exam Band 6.... I felt like I needed to have a break as a reward before moving on to the next goal. (Jasmine)

Moreover, Linnaeus shared her similar experience.

When completing a goal, I might just relax a little bit and then move on to the next phase. (Linnaeus)

Taking a break before moving to the next goal involves interrupting steady and continuous progress that characterizes locomotion. This tendency indicated that they did not have very strong locomotion orientations.

Lack of focus on learning tasks. Three participants reported that they were easily distracted while studying and found it difficult to be fully focused on learning tasks. Sharon recalled that when she practiced her listening exercises on an APP, she always wanted to play on the phone. Linnaeus also reported a similar learning experience. She said,

If I did it attentively, I could finish it in a very short time. The biggest problem was that I played a lot while doing assignments, so it would take much more time to finish. (Linnaeus)

Locomotion is positively related to attention control when engaging in activities (Higgins et al., 2003). These students tended to be easily distracted from their learning tasks, which suggests that their locomotion orientations were not strong.

Alignment with below average promotion. The participants reported very few (and not very strong) examples of approaches to learning and learning-related tasks/activities that 
were likely to be associated with promotion. Two participants discussed some promotion-related reasons for learning at university. In particular, Sharon said that she was motivated to study hard at university by attaining individual growth.

I want to have a better performance on the postgraduate entrance examination, so that I can gain more opportunities in future. (Sharon)

These interview data suggest that the motivation effect of promotion was neither prevalent nor strong for these participants.

Alignment with strong prevention. The approaches to learning and learning-related tasks/activities reported by all four participants align with their strong prevention orientations. Specifically, they worked to fulfill duties and responsibilities, adopted prevention goals, and were motivated by failure and negative feedback. They were also cautious and careful with studies and decision-making at university.

Striving to meet responsibilities and parental expectations. All four interviewees reported that they worked to fulfil their duties to families and meet their parental expectations. They also stated being pressured by living up to their parents' expectations of them.

..they want me to be better in terms of studies at school, daily behaviors and working competence than the others, especially those kids from our relatives'families. (Linnaeus)

These expectations made her feel very anxious, "because I keep telling myself that I have to study hard and live up to their expectations" (Linnaeus).

In addition, two interviewees reported other responsibilities they had to meet. Qian related her internship experience with an insurance company.

I'm responsible for the businesses of three staff there. I do my tasks slowly, for I don't want to make mistakes and fail to meet the job responsibilities. (Qian)

Sharon also mentioned that she had to work hard at English listening.

...this (English listening) is required in my major, and it is very obligatory for me to improve English listening. (Sharon)

Although this course was difficult and boring for her, she could not give up because she had to meet the major requirement. These accounts reflect a strong prevention focus.

Setting prevention-related goals. All four participants reported setting preventionrelated goals. Qian stated some examples of prevention-related goals she set at university such as passing the National College English Band 4 and Band 6 tests. 
Moreover, Jasmine set the similar prevention goals of not failing in some difficult courses such as mathematics. She was motivated by these prevention goals.

There's nothing I could do but learn what I was supposed to learn well, in case I fail the exam. I was scared of failing. (Jasmine)

In addition, Linnaeus aimed to complete a postgraduate programme in the long-term because she saw this as an obligation,

...it feels like the master degree is a must for us (i.e., undergraduate students). (Linnaeus)

For the participants classified in this class, their prevention focus appeared very strong in the contexts of the exams that they did not want to fail. These participants worked hard to meet their responsibilities and attain their prevention goals.

Being cautious and careful. All four participants reported being cautious and careful with their studies and decision-making at university. They tried to protect against negative outcomes. For example, as mentioned previously, Linnaeus confided that she was very cautious especially for important decisions in life, such as the decision about which university to apply to for a postgraduate programme. She wanted to apply for universities in south China, but she found the test questions for the entrance exams in those universities creative and unpredictable. Therefore, she finally decided to apply for a university in the north. She commented,

Anyway, the entrance exam involves a lot of uncertainty. I want to be cautious to secure a higher likelihood to do my postgraduate studies. (Linnaeus)

She tried to be cautious by choosing the target university which had a lower risk of failure in the postgraduate entrance exam.

Moreover, all four students described their strategies of preparing for the exams in order not to fail. They tended to "memorize the key points that the teachers stress in class before the final exams" (Sharon). Linnaeus added,

I reviewed those key points, which secured me most of the scores.

\section{(Linnaeus)}

Furthermore, Qian reported that she wrote down what she had memorized to answer test questions during the examinations. By focusing on these prepared answers, she thought she could pass the exam and secure a satisfactory score.

All four students also reported that they were very careful with their assignments and "afraid of making mistakes" (Sharon). For example, when doing some accounting exercises, 
Jasmine read the instructions carefully and tried to identify all the important information, so that she could avoid mistakes. In addition, she noted,

I was afraid that I made some mistakes during the process, so I had to double check every number in each entry with my partner, to make sure each number was correct. (Jasmine)

Moreover, Sharon said that when preparing for a presentation in class, she did what was necessary to avert mistakes in the presentation.

I thought a lot about potential mistakes I might make in the presentation. I rehearsed it many times in my mind.... I am very careful, afraid of making mistakes. (Sharon)

Qian observed that she started doing her assignment early, but she worked very slowly so as not to make errors.

It took me a pretty long time..... I checked what I did again and again. For example, it is easier to make mistakes in typing, and I had to go over it and check the typing over and over again. (Qian)

These data showed that these participants were careful to guard against negative outcomes and minimize the risks of making mistakes. These tendencies are consistent with a strong prevention orientation.

Being motivated by failure and negative feedback. All four participants reported that they were motivated by failure. For example, Sharon noted that although she felt very sad when she learned about the unsatisfactory result of the English listening exam, she was motivated by this failure to "work harder with a stronger desire to learn" (Sharon). Qian also talked about her different responses to success and failure.

I think failure works better for me. If I fail, I want to conquer it. Success may bring me happiness, but it won't give me the motivation to go on. And failure makes me feel like I have to be successful. Success makes me feel like I've reached my goal. Failure makes me feel like I still have to work harder. (Qian)

She mentioned that success reduced her motivation to expend more effort, while failure strengthened her motivation to work harder. In line with the regulatory focus theory (Higgins, 2012), when individual have a strong prevention focus, success reduces vigilance and, thus, decreases motivation, whereas failure strengthens vigilance, and, thus, increases motivation.

Linnaeus and Qian also thought that failure helped them identify some problems and weaknesses, so that they could try to avoid these problems in the future. In 
Linnaeus' words,

I wanted to learn from this failure, reflecting on whether there was anything wrong with my methods or something. After all, the lesson from this failure can be of help for learning other courses, so I can avoid these problems in future. (Linnaeus)

Similarly, all four students discussed the motivating effect of negative feedback I prefer the negative feedback pointing out the shortcomings. I could get better after correcting them. Pointing out the strength didn't help me become better. It should be the negative feedback that points out my problems has a bigger impact on me. (Qian)

In contrast, positive feedback appeared to reduce the vigilance these participants needed to prevent failure.

Table 37 summarizes the data reported by the four participants recruited from this class for each theme. The quantitative findings showed that the Class 2 profile was characterized by comparable and below average levels of assessment, locomotion, and promotion as well as very high levels of prevention. For this class, prevention dominated in magnitude the other motivation orientations. As I discussed in this section, in general, the approaches to learning and learning-related tasks/activities reported by these interviewees aligned with this motivation profile. 
Table 37

Summary of the Four Participants' Data for Each Theme (C2)

\begin{tabular}{|c|c|c|c|c|c|}
\hline \multirow[t]{2}{*}{ Theme } & \multirow[t]{2}{*}{ Category } & \multicolumn{4}{|c|}{ Participant } \\
\hline & & Jasmine & Linnaeus & Qian & Sharon \\
\hline \multirow[t]{4}{*}{$\begin{array}{l}\text { Alignment } \\
\text { with below } \\
\text { average } \\
\text { assessment }\end{array}$} & $\begin{array}{l}\text { Making critical } \\
\text { comparisons and } \\
\text { evaluations }\end{array}$ & $\begin{array}{l}\text { Compared herself to } \\
\text { her classmates; } \\
\text { Did not evaluate what } \\
\text { she learned in class }\end{array}$ & $\begin{array}{l}\text { Compared herself to } \\
\text { others; } \\
\text { Did not evaluate what } \\
\text { she learned in class }\end{array}$ & $\begin{array}{l}\text { Did not evaluate what } \\
\text { she learned in class }\end{array}$ & $\begin{array}{l}\text { Did not reflect on what } \\
\text { she learned in class }\end{array}$ \\
\hline & Striving for accuracy & $\begin{array}{l}\text { Checked her } \\
\text { assignments for } \\
\text { accuracy }\end{array}$ & $\begin{array}{l}\text { Looked for accurate } \\
\text { information }\end{array}$ & $\begin{array}{l}\text { Checked the format } \\
\text { and slides }\end{array}$ & \\
\hline & Weighing up options & & $\begin{array}{l}\text { Weighed up alternative } \\
\text { universities }\end{array}$ & & $\begin{array}{l}\text { Hesitated about } \\
\text { choosing universities }\end{array}$ \\
\hline & $\begin{array}{l}\text { Reflecting on previous } \\
\text { personal experiences }\end{array}$ & $\begin{array}{l}\text { Reflecting on not } \\
\text { working hard }\end{array}$ & & & $\begin{array}{l}\text { Reflecting on her } \\
\text { choice of majors }\end{array}$ \\
\hline \multirow{2}{*}{$\begin{array}{l}\text { Alignment } \\
\text { with below } \\
\text { average } \\
\text { locomotion }\end{array}$} & Moving to the next goals & $\begin{array}{l}\text { Took a break before } \\
\text { moving to the next } \\
\text { goal }\end{array}$ & $\begin{array}{l}\text { Relaxed before moving } \\
\text { to the next phase }\end{array}$ & $\begin{array}{l}\text { Took a break before } \\
\text { moving to the next } \\
\text { goal }\end{array}$ & $\begin{array}{l}\text { Took a break before } \\
\text { moving to the next } \\
\text { goal }\end{array}$ \\
\hline & $\begin{array}{l}\text { Lack of focus on learning } \\
\text { tasks }\end{array}$ & & $\begin{array}{l}\text { Played while doing her } \\
\text { assignments }\end{array}$ & $\begin{array}{l}\text { Was easily distracted } \\
\text { from learning tasks }\end{array}$ & $\begin{array}{l}\text { Was easily distracted } \\
\text { from doing listening } \\
\text { exercises }\end{array}$ \\
\hline
\end{tabular}




\begin{tabular}{|c|c|c|c|c|c|}
\hline \multirow[t]{2}{*}{ Theme } & \multirow[t]{2}{*}{ Category } & \multicolumn{3}{|c|}{ Participant } & \multirow[b]{2}{*}{ Sharon } \\
\hline & & Jasmine & Linnaeus & Qian & \\
\hline \multirow[t]{4}{*}{$\begin{array}{l}\text { Alignment } \\
\text { with strong } \\
\text { prevention }\end{array}$} & $\begin{array}{l}\text { Striving to meet } \\
\text { responsibilities and } \\
\text { parental expectations }\end{array}$ & $\begin{array}{l}\text { Fulfilled obligations to } \\
\text { family; } \\
\text { Lived up to parental } \\
\text { expectations }\end{array}$ & $\begin{array}{l}\text { Met parental } \\
\text { expectations }\end{array}$ & $\begin{array}{l}\text { Fulfilled } \\
\text { responsibilities at work }\end{array}$ & $\begin{array}{l}\text { Lived up to parental } \\
\text { expectations; } \\
\text { Met the requirement of } \\
\text { her major }\end{array}$ \\
\hline & $\begin{array}{l}\text { Setting prevention-related } \\
\text { goals }\end{array}$ & $\begin{array}{l}\text { Set the goal of not } \\
\text { failing in the final } \\
\text { exams }\end{array}$ & $\begin{array}{l}\text { Did not want to fail in } \\
\text { the final exams; } \\
\text { Had to secure a } \\
\text { postgraduate programme }\end{array}$ & $\begin{array}{l}\text { Set the goal of not } \\
\text { failing in the final } \\
\text { exams; } \\
\text { Had to pass National } \\
\text { English tests }\end{array}$ & $\begin{array}{l}\text { Set the goal of not } \\
\text { failing in the final } \\
\text { exams }\end{array}$ \\
\hline & $\begin{array}{l}\text { Being cautious and } \\
\text { careful }\end{array}$ & $\begin{array}{l}\text { Reviewed the key } \\
\text { points for the final } \\
\text { exams; } \\
\text { Read instructions } \\
\text { carefully; } \\
\text { Checked her } \\
\text { assignments }\end{array}$ & $\begin{array}{l}\text { Reviewed the key points } \\
\text { for the final exams; } \\
\text { Was cautious when } \\
\text { applying for a } \\
\text { postgraduate } \\
\text { programme; } \\
\text { Checked her } \\
\text { assignments }\end{array}$ & $\begin{array}{l}\text { Reviewed the key } \\
\text { points for the final } \\
\text { exams; } \\
\text { Answered the test } \\
\text { questions as prepared; } \\
\text { Checked the typing }\end{array}$ & $\begin{array}{l}\text { Reviewed the key } \\
\text { points for the final } \\
\text { exams; } \\
\text { Was careful not to } \\
\text { make mistakes }\end{array}$ \\
\hline & $\begin{array}{l}\text { Being motivated by } \\
\text { failure and negative } \\
\text { feedback }\end{array}$ & $\begin{array}{l}\text { Felt motivated by } \\
\text { failure and negative } \\
\text { feedback }\end{array}$ & $\begin{array}{l}\text { Felt motivated by } \\
\text { negative feedback }\end{array}$ & $\begin{array}{l}\text { Felt motivated by } \\
\text { failure and negative } \\
\text { feedback }\end{array}$ & $\begin{array}{l}\text { Felt motivated by } \\
\text { failure }\end{array}$ \\
\hline
\end{tabular}




\section{Case 3: Participants with the C3 Profile}

Four themes emerged from the qualitative data provided by the three participants who had the C3 profile (i.e., Quan, Vivian, and Zi). These themes are: "alignment with strong assessment", "alignment with strong locomotion", "alignment with strong promotion" and "alignment with weak prevention".

Alignment with strong assessment. The approaches to learning and learningrelated tasks/activities reported by all three participants recruited from this class are consistent with their strong assessment orientations. Specifically, they tended to make critical comparisons and evaluations, think carefully before taking action, and draw on previous behaviors.

Making critical comparisons and evaluations. All three participants reported comparing and evaluating the theoretical knowledge so that they could have a clear understanding of what they learned in their courses. Zi cited her leaning experience as an example.

... in one class, we learned four theories. I put them into a table, listing things such as the time they were put forward, notable figures, research perspectives and methodologies. ...It's easier for me to make a thorough understanding of these theories. $(\mathrm{Zi})$

Moreover, Quan mentioned that he compared different assertions he learned in class or online to find out their similarities and differences and evaluated them to see if they were convincing.

In addition, two students described their self-evaluative concerns by comparing themselves to others. As Vivian said,

Some classmates living in my neighboring dorms usually went back at 11 pm and they had been studying hard all the time. I thought, "Oh, they must have been better prepared than me and will do better than me in the exam. (Vivian)

Similarly, Zi reported that she sometimes unconsciously compared her academic performance to that of her friends and classmates. She felt "a sense of loss" when she found the others did better. This self-evaluative concern is characteristic of a strong assessment orientation (Higgins et al., 2003).

Thinking thoroughly before taking action. Two students noted that they thought 
carefully before doing their work. In particular, Quan mentioned,

I have to think about how to do it, like, what are the best ways and what are the standards I want to meet. (Quan)

Moreover, $\mathrm{Zi}$ reported that she gave careful consideration to the value of the goals she wanted to achieve at university. She noted,

I have a lot of options for my university life. But I have to think carefully which are most important for me in different phases. For instance, I think that getting a master's degree is the most crucial goal at present, so I will prioritize the work related to the postgraduate entrance exam. (Zi)

These participants tended to work towards the options that offered the highest potential value, which is another key feature of strong assessment. Finally, Zi mentioned that she was working for several goals at the same time.

I have been mainly preparing for the postgraduate entrance exam. But I have been also working for other goals like the teaching certificate, but with limited time and effort. ( $\mathrm{Zi})$

Thus, it is likely that her strong assessment orientation enabled her to identify a set of worthwhile goals that could be pursued simultaneously.

These findings show that the three participants expended time and effort identifying important goals and seeking effective means to achieve these goals. These tendencies are consistent with a strong assessment orientation (Higgins et al., 2003).

Reflecting on previous experiences. Quan reported that he tended to reflect on what he had done. He confided that after he failed, he would spend a whole day reflecting on his failure.

I thought a lot about why I failed and what I should have done. (Quan) Moreover, he said that after he achieved one goal, he preferred to reflect on what he had done.

I tried to identify what I had done right and wrong. I also summed up my personal strengths and weaknesses from this experience. (Quan)

In addition, when Vivian recalled her experience of preparing for the postgraduate entrance exam, she expressed her regret about her previous behaviors.

If I had reviewed the test papers in the past years and worked much harder, I would have succeeded in the exam. (Vivian)

She said that reflection on this past experience enabled her to identify the best way to prepare for the entrance exam next time. Reflection on previous experiences helped these 
participants identify what they needed to improve. These accounts align with a strong assessment orientation.

Alignment with strong locomotion. The approaches to learning tasks/activities reported by these participants align with the strong locomotion that characterized their motivation profile. Specifically, to maintain smooth flow of work, they preferred to do things step by step, moved on to the next goals, were fully focused on learning tasks, preferred speed over accuracy and engaged in timely action.

Doing things step by step. Two participants discussed that they preferred to do their assignments step by step. In particular, Quan said,

I like to do things in a neatly organized way, moving to another task after finishing one according to my schedule. I enjoy the feeling of flow at work. (Quan)

He said that this flow could ensure the efficiency of his work. In addition, $\mathrm{Zi}$ also described how she followed steps to complete her essay.

Once I started, I moved forward smoothly, searching for references, sorting out ideas and writing the assignment. One phase finished, I moved to the next step. $(\mathrm{Zi})$

She maintained steady movement from one phase to another, which is consistent with a strong locomotion orientation (Kruglanski et al., 2000).

Giving up original goals when experiencing no progress. All three participants reported that they tended to abandon their original goals and strive for new ones when they found that no progress was made. Quan confided,

I participated in a contest. When I felt the contest was beyond my competence, or I wouldn't make any progress, I quit and moved on to find out something else more suitable to me. (Quan)

In addition, $\mathrm{Zi}$ also wanted to strive for new goals to make sure that she made progress.

I don't want to get stuck by sticking to one goal. If I fail (in the postgraduate entrance exam), I will take the recruitment exams for civil servants or for teachers in public schools. $(\mathrm{Zi})$

Giving up original goals and working towards new goals can ensure steady and continuous progress, which is consistent with strong locomotion.

Being focused on learning tasks. Two participants reported that they were fully focused on their learning tasks. Quan said, 
I had a strong self-control in studies. I was focused on my task at hand and not easily distracted. (Quan)

$\mathrm{Zi}$ also noted that if she wanted to do it, she would be focused on it, not interrupted by distractions. She said,

I don't like to be interrupted and want to make steady progress till the completion of the task. (Zi)

Locomotion is positively related to attention when engaging in activities. These reports are, thus, consistent with a strong locomotion orientation. In contrast, Vivian reported that she tended to be distracted when doing her assignments. This data suggests that locomotion was likely to be less chronically accessible to her than assessment.

Preference for speed. Two students reported their preference for speed when they worked on their assignments. For instance, Zi noted that once she started doing her English writing task, she finished it within a very short period of time.

I put my ideas on paper quickly. I didn't stop frequently to check my spellings or grammar. I just wanted to move forward fast till completion.

Additionally, Vivian also preferred to finish her assignments quickly. She did not pay much attention to some details such as the format of the essay, because "checking these details would make me slow down in the process of writing" (Vivian). Focusing on speed rather than accuracy enabled them to maintain quick movement in doing their learning tasks, which is consistent with a strong locomotion orientation.

"I am a doer". Quan reported that he was "a doer". He said that he preferred to take action immediately. He observed,

It's my personality. I will do immediately what I think. ... Take the survey I conducted as an example. When the topic came to me, I couldn't wait to conduct the survey. Very excited to get started, and to apply what I learned in class to practice. (Quan)

For this interviewee, it was likely that locomotion, which is negatively related to procrastination (Pierro et al., 2011), was stronger or more chronically accessible than assessment. In contrast, Vivian and Zi reported that they tended to procrastinate in doing their assignments, starting to work on them only several days before the deadlines. This tendency is consistent with the quantitative findings that assessment was also strong for the participants from Class 3; perhaps assessment was more chronically accessible to these two participants than was locomotion. 
Alignment with strong promotion. The interview data from the respondents selected from Class 3 suggested that all three participants had tendencies that are consistent with a strong promotion orientation. Specifically, they worked to achieve their hopes and individual growth, were motivated by success and positive feedback, and were enthusiastic with learning at university.

Striving for ideals. All three participants reported promotion-related reasons that motivated them to work hard at university. Specifically, they strived to achieve individual aspirations and growth. For example, Vivian viewed her goal of pursuing a postgraduate programme in her dream university as an aspiration she worked hard to achieve. In addition, $\mathrm{Zi}$ said that she dreamed to be a teacher and had been working hard to realize this dream. Moreover, she noted that she wanted to improve her individual growth rather than meet her parents' expectations.

My father wanted me to learn accounting or finance... But I preferred to be a teacher...I still want to work for my individual development by following my own interest. ( $\mathrm{Zi})$

Another example of attaining individual growth was reported by Quan.

He saw pursuing a master degree as an opportunity for individual growth. He commented, I won't pursue it for the sake of getting a degree, but to improve myself academically and professionally. (Quan)

These accounts are consistent with a strong promotion orientation.

Being motivated by success and positive feedback. All three participants stated that they were motivated by success and positive feedback.

Success will definitely motivate me better, because success is an affirmation of myself. For example, when I got the scholarships and the second prize in the social research competition, I felt very happy and proud of my achievements. (Vivian)

Another example of the motivating effect of success was reported by $\mathrm{Zi}$.

Success motivates me to move forward. ... When thinking about my previous successes, I feel more confident and happy. I think that the difficulty isn't a big deal because I have done well in the past. (Zi)

Apart from being motivated by success, all three interviewees reported that they felt motivated by positive feedback.

Positive feedback means my competence is recognized by them. People 
believe in me, and thus I believe in myself and work harder. $(\mathrm{Zi})$

In contrast, all three participants reported that when experiencing failure, they felt "in low spirits, with the failure like a dark cloud over me" (Quan), "upset" (Vivian) and "depressed for a while" (Zi). Zi also commented that she tended to give up when receiving negative feedback.

Consistent with their promotion orientations, the motivation of these students was strengthened by success and positive feedback. In contrast, their motivation was reduced by failure and negative feedback.

Being enthusiastic. All three participants reported that they were keen to strive for advancement. For instance, Quan mentioned that he preferred to read at a fast pace when doing English reading comprehension tests.

I wanted to go through all the passages in the required time so that I could gain more scores. If I read the passages slowly, I couldn't complete all the questions. (Quan)

$\mathrm{Zi}$ also reported that she preferred to read quickly the English passages in English tests. She commented,

Only in this way can I get as much available information as possible to answer the comprehension questions. (Zi)

They utilized the strategy of speed to maximize their opportunities to answer all the questions in the limited time. Thus, these examples illustrate these students' preference for eager strategies.

In addition, Vivian shared her experience of reading beyond requirements and consulting with both peers and the teacher; these are important facets of using eager strategies in learning settings (Molden \& Rosenzweig, 2016).

I tried to read more books beyond the course requirement. I discussed with the teacher after class sometimes. I also talked with my classmate.....

Anyway, I was eager to learn more. I felt great about it. (Vivian)

Finally, Zi confided that she was very positive towards life. She observed,

I'm very optimistic. I am upset when faced up with some difficulty, but I can adjust my mood quickly. I think I can make it because I have done well in the past. (Zi)

Their optimistic outlooks and beliefs in their personal strengths enabled them to persist in overcoming difficulties and strive for advancement. 
Alignment with weak prevention. Some of the approaches to learning and learningrelated tasks/activities reported by these interviewees did not align with their weak prevention. In particular, the three students reported adopting some prevention-related goals at university. Quan said that he had to do some courses he found boring because they were required courses at university and he tried hard not to fail the exams. $\mathrm{Zi}$ also mentioned that she had to get the certificate of international teaching because "this certificate is the essential requirement if you want to apply for the Hanban-financed voluntary program of teaching Chinese in foreign countries" $(\mathrm{Zi})$.

In contrast, two students reported a relative lack of carefulness. Vivian mentioned that after finishing her assignments, she checked what she did, "but not very carefully." (Vivian)

I am a careless person. For instance, if the mistake in the format of my paper didn't affect the overall effect, I wouldn't bother to correct it. I don't pay attention to these small mistakes. (Vivian)

This tendency of being careless is consistent with a weak prevention focus.

On the other hand, Quan said when collecting the paper questionnaires, he had to check the questionnaires carefully to ensure the quality of the data collected. These participants' responses suggest that prevention was likely to be more chronically accessible to Quan than to the other respondents. Quan also noted that he was motivated more by failure and negative feedback.

I feel very happy when I succeed, but I might get too happy and even a bit arrogant, which keep me from reflecting on what I have done.... Failure will enable me to identify my weakness so that I can try my best to make it next time. Failure or negative feedback motivate me to more efforts. (Quan)

His prevention focus was complemented by his strong assessment concerns, which enabled him to reflect on his failure experiences and avoid negative outcomes in future.

These reports are not consistent with the weak prevention that characterized the Class 3 profile. A potential explanation could be that the participants' prevention was primed in the contexts of examinations. Intriguingly, Quan appeared to display some tendencies that were related to a strong prevention orientation.

Table 38 sums up the data reported by the three participants for each theme. The quantitative findings showed that the Class 3 profile was characterized by having strong/very strong and comparable assessment, locomotion, and promotion, but very weak prevention. As I discussed in this section, the interview data from Class 3 suggest that the approaches to learning and learning-related tasks/activities 
mapped well onto the characteristics of the $\mathrm{C} 3$ profile, except for some tendencies related to prevention. 
Table 38

Summary of the Three Participants' Data for Each Theme (C3)

\begin{tabular}{|c|c|c|c|c|}
\hline \multirow[t]{2}{*}{ Theme } & \multirow[t]{2}{*}{ Category } & \multicolumn{3}{|c|}{ Participant } \\
\hline & & Quan & Vivian & $\mathrm{Zi}$ \\
\hline \multirow[t]{3}{*}{$\begin{array}{l}\text { Alignment } \\
\text { with strong } \\
\text { assessment }\end{array}$} & $\begin{array}{l}\text { Making critical comparisons and } \\
\text { evaluations }\end{array}$ & $\begin{array}{l}\text { Compared and evaluated } \\
\text { different assertions }\end{array}$ & $\begin{array}{l}\text { Compared different theories; } \\
\text { Compared herself to her } \\
\text { classmates }\end{array}$ & $\begin{array}{l}\text { Compared different } \\
\text { theories; } \\
\text { Compared herself to her } \\
\text { classmates }\end{array}$ \\
\hline & $\begin{array}{l}\text { Thinking thoroughly before } \\
\text { taking action }\end{array}$ & $\begin{array}{l}\text { Thought carefully before } \\
\text { doing the leaning-related } \\
\text { tasks; } \\
\text { Evaluated different goals }\end{array}$ & & $\begin{array}{l}\text { Evaluated different goals; } \\
\text { Identified a set of } \\
\text { worthwhile goals to strive } \\
\text { for at the same time }\end{array}$ \\
\hline & $\begin{array}{l}\text { Reflecting on previous } \\
\text { experiences }\end{array}$ & $\begin{array}{l}\text { Reflected on his previous } \\
\text { experiences }\end{array}$ & $\begin{array}{l}\text { Regretted what she had done } \\
\text { to prepare for the postgraduate } \\
\text { entrance exam }\end{array}$ & \\
\hline \multirow{2}{*}{$\begin{array}{l}\text { Alignment } \\
\text { with strong } \\
\text { locomotion }\end{array}$} & Doing things step by step & $\begin{array}{l}\text { Did things in an organized } \\
\text { way }\end{array}$ & & $\begin{array}{l}\text { Did her assignments step by } \\
\text { step }\end{array}$ \\
\hline & Being focused on learning tasks & Was concentrated on his task & $\begin{array}{l}\text { Was easily distracted from her } \\
\text { learning tasks }\end{array}$ & $\begin{array}{l}\text { Did not like interruptions } \\
\text { when doing her assignments }\end{array}$ \\
\hline
\end{tabular}




\begin{tabular}{|c|c|c|c|c|}
\hline \multirow[t]{2}{*}{ Theme } & \multirow[t]{2}{*}{ Category } & \multicolumn{3}{|c|}{ Participant } \\
\hline & & Quan & Vivian & $\mathrm{Zi}$ \\
\hline \multirow{5}{*}{$\begin{array}{l}\text { Alignment } \\
\text { with strong } \\
\text { promotion }\end{array}$} & Preference for speed & & $\begin{array}{l}\text { Finished her assignments } \\
\text { quickly }\end{array}$ & Completed her writing fast \\
\hline & "I am a doer" & Initiated action immediately & Procrastinating & $\begin{array}{l}\text { Doing her assignments } \\
\text { before the deadline }\end{array}$ \\
\hline & Striving for ideals & $\begin{array}{l}\text { Studied for individual } \\
\text { growth }\end{array}$ & Studied to achieve aspirations & $\begin{array}{l}\text { Studied to achieve her } \\
\text { dream and growth }\end{array}$ \\
\hline & $\begin{array}{l}\text { Being motivated by success and } \\
\text { positive feedback }\end{array}$ & $\begin{array}{l}\text { Felt motivated by success } \\
\text { and positive feedback }\end{array}$ & $\begin{array}{l}\text { Felt motivated by success and } \\
\text { positive feedback }\end{array}$ & $\begin{array}{l}\text { Felt motivated by success } \\
\text { and positive feedback }\end{array}$ \\
\hline & Being eager and optimistic & $\begin{array}{l}\text { Used the strategy of speed to } \\
\text { gain more points; } \\
\text { Was optimistic when she } \\
\text { faced difficulties }\end{array}$ & $\begin{array}{l}\text { Learned beyond the course } \\
\text { requirement; } \\
\text { Asked teachers and } \\
\text { classmates for help }\end{array}$ & $\begin{array}{l}\text { Used the strategy of speed } \\
\text { to gain more available } \\
\text { information; } \\
\text { Was optimistic }\end{array}$ \\
\hline $\begin{array}{l}\text { Alignment } \\
\text { with weak } \\
\text { prevention }\end{array}$ & & $\begin{array}{l}\text { Had prevention-related goals; } \\
\text { Was careful; } \\
\text { Felt motivated by failure and } \\
\text { negative feedback }\end{array}$ & $\begin{array}{l}\text { Had prevention-related goals; } \\
\text { Was careless }\end{array}$ & $\begin{array}{l}\text { Had prevention-related } \\
\text { goals; } \\
\text { Was careless }\end{array}$ \\
\hline
\end{tabular}




\section{Case 4: Participants with the C4 Profile}

Four themes emerged from the qualitative data provided by the three participants who exhibited the $\mathrm{C} 4$ profile (i.e., Kiva, Mona, and Tian). These themes are "alignment with weak assessment", “alignment with weak locomotion", "alignment with weak promotion", and "alignment with below average prevention".

Alignment with weak assessment. These participants reported some approaches to learning and learning-related tasks/activities that align with their weak assessment orientations. Specifically, they did not evaluate the knowledge they learned and did not think about goals at university and ways to achieve the goals. Notably, they also tended to compare themselves to other people and reflect on their previous experiences; both of these tendencies are typically associated with strong assessment.

Making critical comparisons and evaluations. All three participants mentioned that they did not critically evaluated the knowledge they learned in their courses. As Tian confided,

I am not motivated to evaluate theories or assertions the teacher taught in class or we read from the textbooks. I just tried to memorize what we were taught. (Tian)

This tendency is consistent with a weak assessment orientation.

However, two participants reported that they tended to compare themselves to people around them and that this comparison caused them negative emotions. For example, Tian recalled the experience of struggling with a mathematics course. She compared her performance in this course to that of her classmates and felt, as a result, "very upset and disappointed".

I wanted to cry and wondered why all but me could do it. Some classmates who did poorly in the last exam could work out the questions. I thought I wasn't smart, and wasn't suitable for learning this course. (Tian)

Experiencing these negative emotions, she was on the verge of quitting. In addition, Kiva noted that she tended to unconsciously compare herself to those excellent young people she met. She felt frustrated and thought that she was not smart compared to these people. These account are consistent with having a strong assessment orientation.

Reflecting on previous experiences. Two participants reported that they reflected on their previous experiences. Tian confided that every night before going to bed, she reflected 
on what she had done during the day.

I feel pretty sorry that I haven't followed my plan and decide to work harder next day. But in fact, I do not make any changes the following days. Then I feel guilt and regret again. I can't break this cycle. (Tian)

This account suggests that she knew what she needed to do, but took no action.

In addition, when Mona recalled her disengagement in studies in the past two years at university, she expressed her remorse for her behaviors.

I feel regretful for what I have done. If I had studied hard in the first two years, I would have less pressure now. If only I hadn't wasted so much time!

(Mona)

Reflecting on what would have happened if she did it right made her feel remorse for her previous behaviors. These tendencies of thinking about what was the right course of action are consistent with a strong assessment orientation.

Identifying goals and means to achieve the goals. Mona noted that during her first two years at university, she seldom gave careful consideration to goals she could pursue at university and to finding effective means to attain the goals.

I wasn't engaged in studies at that time. I didn't think about any goals in learning. After failing in switching to another major in my first year, I thought that I might pursue postgraduate studies. But it was years away. I didn't think about how to prepare for the postgraduate exam. (Mona) This report is consistent with a weak assessment orientation.

These qualitative data suggest that these participants showed tendencies that are related to both weak and strong assessment orientations. This reported approach to learning does not align with the weak assessment that characterized their motivation profile.

Alignment with weak locomotion. The approaches to learning and learning-related tasks/activities reported by the participants align with their weak locomotion orientations. Specifically, they tended to procrastinate and were easily distracted from learning tasks.

Procrastinating. Two interviewees reported that they tended to procrastinate rather than take timely action.

I don't do it unless somebody reminds me or the deadline is close. I tend to procrastinate. It's a very bad habit, but I can't get out of it. I am not motivated to do things. I need others to push me. (Tian)

She reported that she lacked motivation to initiate action and change. Mona also stated that she tended to work on an assignment at the last minute before the deadline during the first 
two years at university. As procrastination is negatively related to locomotion (Pierro et al., 2011), these accounts are consistent with a weak locomotion orientation.

In contrast, Kiva reported that she tended to do her assignment on the day when the teacher assigned it.
I don't procrastinate. It's my way of doing things: springing to action quickly. I'm afraid I can't submit my assignment on time if I don't start early. (Kiva)

Her account suggests that the prevention focus activated by the assignment motivated her to take timely action, despite having low levels of locomotion.

Being distracted from learning tasks. All three participants reported that they were easily distracted while studying and found it difficult to focus on their learning tasks.

When I was doing my homework, some friend showed me something interesting, and I put my homework aside, went playing with her, and forgot about my homework. When I resumed doing it, I was still easily distracted. (Tian)

Kiva and Mona also reported that they were distracted by things such as their mobile phones. Considering that locomotion is positively related to maintaining task focus and resisting distractions (Higgins et al., 2003), these accounts align with the weak locomotion orientation that characterized these respondents' motivation profile.

Alignment with weak promotion. In contrast with the weak promotion that characterized their motivation profile, the interview data from Class 4 suggested that two of the participants showed characteristics associated with a strong promotion orientation. Specifically, they reported that they strived to realize their dreams, achieve accomplishments and were motivated by success.

Working to achieve aspirations and accomplishments. Two participants reported that promotion-related reasons motivated them to study at university. For example, Mona was preparing for the postgraduate entrance exam this year. She regarded a master's degree as "an accomplishment of academic growth". In addition, Tian noted that her passion for English learning was partly fueled by her childhood dream to be a diplomat. Both of these participants worked to attain their aspirations and accomplishments, which characterize promotion-related motivation.

Being motivated by success and demotivated by failure. Two students reported that success motivated them to study harder, while failure weakened their motivation. Tian observed that her love for English was largely due to her previous successful experiences of 
English learning. She also viewed success as a recognition of her competence, which motivated her to work harder to "win more recognitions". In contrast, she lost interest in accounting because of her failure in a test for an accounting certificate.

I was aspiring for success at that time and took a lot of tutoring classes for

this exam, but I failed. This failure told me that I wasn't suitable to accounting, so I gave up. (Tian)

Mona also noted that she felt depressed and demotivated when she failed in the exam to transfer to other majors in her first year at university. Their responses to success and failure are consistent with a strong promotion focus.

Alignment with below average prevention. The three participants reported approaches to learning and learning-related tasks/activities that are associated with both strong and weak prevention orientations. In particular, they tried to meet parental expectations, maintain safety and adopted prevention-related goals. They also reported following the course requirements carefully. Notably, they discussed different strategies to prevent mistakes.

Working to fulfil responsibilities. The three participants reported that they worked to fulfil their responsibilities to families and live up to parental expectations. For instance, Mona noted that the most important motivation for her was to fulfil obligations to her family. She observed,

I was brought up by my grandparents. I have to pay them back. My mum is

a single mother. She has sacrificed a lot for me. I think that it's my responsibility to give her a better life. (Mona)

She also mentioned that her family had high expectations of her, because it was very difficult for young people in her hometown to go to university. She was driven by their expectations to study hard in high school. Now she viewed pursuing a master's degree as her duty to meet her family's expectation.

Similarly, Kiva reported that her decision to apply for accounting as her major at university was partly influenced by her father's expectations of her.

My dad was a treasurer in a company. He has done a wonderful job, but he was pretty sorry that he didn't learn this major at university. So he wanted me to major in accounting. I thought I had to meet his expectations. (Kiva) These participants worked to fulfil their responsibilities to families and live up to the parental expectations, which is typical of prevention-related motivation.

Working to maintain safety. Two participants reported that they worked to maintain 
safety. For instance, Kiva talked about her reason for choosing accounting as her major. She thought that accounting was "a safe choice", because accounting would secure her future employment after graduation. Apart from securing a good job, Mona also mentioned that she had a high score in the college entrance exam, so she worked hard in the first semester at university to maintain her good academic performance. These accounts reflect a strong prevention focus. Intriguingly, the same student confided that she did not care about maintaining her academic performance in her second year at university.

I did very poorly in the final exams and ranked much lower in my class, but

I didn't care. I wasn't motivated to work hard to maintain my previous ranking. (Mona)

This data could suggest that her prevention focus was not strong at that time (i.e., in her second year at university). The motivational effect of prevention appeared to be more prevalent for her in the third year, when the postgraduate exam was closer.

Adopting prevention-related goals. All three participants discussed their prevention-related goals. For example, Tian said that she did not give up the difficult parts in a mathematics course because she "had to pass the final exam". Mona aslo reported her prevention goal of securing a postgraduate programme.

It's difficult to be a teacher teaching foreigners Chinese if you have a bachelor's degree. You have to have a master's or doctor's degree. It is a must. (Mona)

Their prevention concerns were strong in the contexts of the exams, which they did not want to fail.

Being cautious and careful. All three participants reported that they were cautious with their studies at university. For instance, in order not to fail in the exams, they followed the course requirements and reviewed the materials before the exams. Kiva said that in a course she found difficult, she listened to the teacher attentively in class and memorized diligently the key points highlighted by the teacher. Tian also reported that, in the weeks before the final exam, she spent a lot of time doing the required mathematics exercises that might be tested.

Moreover, Mona related her similar learning experiences in preparing for the postgraduate exam. Specifically, in order to secure a satisfactory score, she attended all the classes related to the exam and completed the work the teacher assigned. She also bought all the required textbooks for the exam and read them carefully.

In addition to following the course requirements, Kiva reported that she was careful 
with her assignments and tried to make sure that she made no mistakes.

I paid particular attention to spellings and grammar. I didn't want to make mistakes and lose marks for being careless. (Kiva)

In contrast, Tian described herself as being careless. She commented, I don't check my work most of the time. I don't have a high standard for my work, and think that it's Ok doing that way. (Tian)

Mona also recalled that she did her assignments carelessly during the first two years. She started the day before the deadline, copied some related materials online and submitted without checking. These tendencies of being careless are consistent with a weak prevention focus.

Being motivated by failure. Kiva reported being motivated by failure. She mentioned that she felt "anxious, under a lot of pressure" when she failed to complete her plans. These emotions motivated her to vigilantly follow her plans. In addition, she mentioned that she was sensitive to failure-related information.

Failure can help me accumulate some experience, so that I will deal with the similar problem carefully next time. I will feel happy with success, but nothing else will be gained. (Kiva)

Notably, she paid particular attention to negative information signaled by failure. This sensitivity enabled her to prevent undesired outcomes in the future. This tendency is consistent with a strong prevention orientation.

Table 39 presents a summary of the data reported by the three participants for each theme. The quantitative findings indicated that the Class 4 profile was characterized by having low/very low levels of assessment, locomotion, and promotion and below average levels of prevention. Prevention was much stronger than any of the other motivation orientations for this class. As I discussed in this section, the reported approaches to learning and learning-related tasks/activities suggest that these students displayed tendencies that aligned with the characteristics of the $\mathrm{C} 4$ profile as well as tendencies that were not consistent with this motivation profile. 
Table 39

Summary of the Three Participants' Data for Each Theme (C4)

\begin{tabular}{|c|c|c|c|c|}
\hline \multirow[t]{2}{*}{ Theme } & \multirow[t]{2}{*}{ Category } & \multicolumn{3}{|c|}{ Participant } \\
\hline & & Kiva & Mona & Tian \\
\hline \multirow[t]{3}{*}{$\begin{array}{l}\text { Alignment } \\
\text { with weak } \\
\text { assessment } \\
\text { orientations }\end{array}$} & $\begin{array}{l}\text { Making critical comparisons and } \\
\text { evaluations }\end{array}$ & $\begin{array}{l}\text { Compared herself to other } \\
\text { people; } \\
\text { Did not evaluate the } \\
\text { knowledge she learned in her } \\
\text { courses }\end{array}$ & $\begin{array}{l}\text { Did not evaluate the } \\
\text { knowledge she learned in her } \\
\text { courses in the first two years } \\
\text { at university }\end{array}$ & $\begin{array}{l}\text { Compared herself to her } \\
\text { classmates; } \\
\text { Did not evaluate the } \\
\text { knowledge she learned in } \\
\text { her courses }\end{array}$ \\
\hline & $\begin{array}{l}\text { Reflecting on previous } \\
\text { experiences }\end{array}$ & & Regretted previous behaviors & $\begin{array}{l}\text { Reflected on previous } \\
\text { experience; } \\
\text { Regretted not taking action }\end{array}$ \\
\hline & $\begin{array}{l}\text { Identifying goals and means to } \\
\text { attain the goals }\end{array}$ & & $\begin{array}{l}\text { Did not think carefully about } \\
\text { goals and how to achieve the } \\
\text { goals during the first two } \\
\text { years }\end{array}$ & \\
\hline \multirow{2}{*}{$\begin{array}{l}\text { Alignment } \\
\text { with weak } \\
\text { locomotion } \\
\text { orientations }\end{array}$} & Procrastinating & $\begin{array}{l}\text { Did her assignments } \\
\text { immediately }\end{array}$ & $\begin{array}{l}\text { Procrastinated during the first } \\
\text { two years }\end{array}$ & Procrastinated \\
\hline & $\begin{array}{l}\text { Being distracted from learning } \\
\text { tasks }\end{array}$ & Was distractible & Was distractible & Was easily distracted \\
\hline
\end{tabular}




\begin{tabular}{|c|c|c|c|c|}
\hline \multirow[t]{2}{*}{ Theme } & \multirow[t]{2}{*}{ Category } & \multicolumn{3}{|c|}{ Participant } \\
\hline & & Kiva & Mona & Tian \\
\hline \multirow{2}{*}{$\begin{array}{l}\text { Alignment } \\
\text { with weak } \\
\text { promotion } \\
\text { orientations }\end{array}$} & $\begin{array}{l}\text { Working for aspirations and } \\
\text { accomplishments }\end{array}$ & $\begin{array}{l}\text { Studied for a master's degree } \\
\text { to achieve accomplishments }\end{array}$ & & $\begin{array}{l}\text { Studied English to attain } \\
\text { aspirations }\end{array}$ \\
\hline & $\begin{array}{l}\text { Being motivated by success and } \\
\text { demotivated by failure }\end{array}$ & & $\begin{array}{l}\text { Felt motivated by success; } \\
\text { Felt demotivated by failure }\end{array}$ & $\begin{array}{l}\text { Felt motivated by success; } \\
\text { Felt demotivated by failure }\end{array}$ \\
\hline \multirow{5}{*}{$\begin{array}{l}\text { Alignment } \\
\text { with below } \\
\text { average } \\
\text { prevention } \\
\text { orientations }\end{array}$} & Working to fulfil responsibilities & Met parental expectations & $\begin{array}{l}\text { Fulfilled family obligations; } \\
\text { Lived up to parental } \\
\text { expectations }\end{array}$ & \\
\hline & Maintaining safety & $\begin{array}{l}\text { Worked to secure future } \\
\text { employment }\end{array}$ & $\begin{array}{l}\text { Pursuing a master's degree to } \\
\text { secure jobs; } \\
\text { Studied to maintaining her } \\
\text { academic performance in the } \\
\text { first year, but not in the second } \\
\text { year }\end{array}$ & \\
\hline & $\begin{array}{l}\text { Adopting prevention-related } \\
\text { goals }\end{array}$ & $\begin{array}{l}\text { Set goals of not failing in the } \\
\text { final exams and getting the } \\
\text { certificate }\end{array}$ & $\begin{array}{l}\text { Set goals of not failing in the } \\
\text { final exams and } \\
\text { securing the admission into a } \\
\text { postgraduate programme }\end{array}$ & $\begin{array}{l}\text { Set goals of not failing in } \\
\text { the final exams }\end{array}$ \\
\hline & Being cautious and careful & $\begin{array}{l}\text { Followed the course } \\
\text { requirements; } \\
\text { Checked the work carefully }\end{array}$ & $\begin{array}{l}\text { Started doing her assignments } \\
\text { early now, but not in the past } \\
\text { two years; } \\
\text { Submitted the assignments } \\
\text { without checking }\end{array}$ & $\begin{array}{l}\text { Followed the course } \\
\text { requirement; } \\
\text { Was careless with her work }\end{array}$ \\
\hline & Being motivated by failure & & & Felt motivated by failure \\
\hline
\end{tabular}




\section{Case 5: Participants with the C5 Profile}

Four themes emerged from the qualitative data provided by the five participants who had the C5 profile (i.e., Dellla, Ivy, Jacky, Jenny, and Wu). These themes are: "alignment with below average assessment", "alignment with below average locomotion", "alignment with below average promotion" and "alignment with below average prevention".

Alignment with below average assessment. The learning approaches reported by these participants suggest that they had tendencies associated with both strong and weak assessment orientations. Specifically, they sometimes critically compared and evaluated the theories they found interesting or important and reflected on their previous experiences; these tendencies are related to strong assessment. In contrast, they did not expend effort in identifying optimal goals, which is associated with weak assessment.

Making critical comparisons and evaluation. All participants reported that they sometimes compared the theories they learned in their courses they found interesting or important. Della cited her learning experience in an economics course as an example.

...there were some different schools in economics. I classified them into different categories, comparing their similarities and differences. (Della)

Engagement in critical comparisons and evaluations of the knowledge acquired/presented is a tendency that is consistent with a strong assessment orientation. However, Della also noted that she accepted and memorized those theories she was not interested in. This tendency is associated with weak assessment.

Apart from critically comparing theories they learned, two students reported that they tended to compare themselves to their classmates and friends.

I feel that I have grown up in such an environment, so I unconsciously compare with others. Sometimes I feel smug because I'm better than others. Sometimes I can feel inferior. (Jenny)

This tendency, which is associated with a strong assessment orientation, rendered these students vulnerable to some negative emotions.

Reflecting on previous experiences. Two participants reported reflecting on their previous experiences. For example, Wu said that when she spent a lot of time reviewing, but made little progress, she would stop and reflect on what she had done.

I tried to find out what went wrong with my learning approaches or something. (Wu) 
Similarly, Della mentioned that she often reflected on her experiences in the past.

I think about my personal strengths and weaknesses. I also try to find what I really want and value in my life. (Della)

Reflection on their previous experiences helped these students identify what they needed to improve to do things right in the future. This tendency is consistent with a strong assessment orientation.

Identifying optimal goals. Three participants reported that they rarely thought about optimal goals they could pursue at university.

I don't have clear long-term goals. I still feel at loss about the future. I don't know what to do in the next years at university. (Ivy)

Jenny also confided that she seldom gave consideration to important goals in her first year and did things aimlessly. This tendency is consistent with a weak assessment.

Alignment with below average locomotion. The interview data from this class illustrated that the participants showed characteristics related to both strong and weak locomotion orientations. Specifically, they preferred to do things step by step and tended to abandon original goals when experiencing little or no progress; both of these tendencies are associated with strong locomotion. They also reported that they did not maintain attention during task engagements, which reflects weak locomotion.

Doing things step by step. Three participants reported that they preferred to do their assignments step by step. For example, Jenny followed several steps when writing her essay.

I collected the related information first. The second step was to make an outline. Then guided by the outline, I put my ideas on paper. After finishing the first draft, I read it carefully and polished up some parts. The last step was to write the essay down by hand. (Jenny)

Ivy also noted that she enjoyed doing mathematics or accounting exercises because she liked the sense of flow, working out the questions step by step. By doing their assignments step by step, these students maintained smooth and steady movement towards their goals, which characterizes a strong locomotion orientation.

Doing something new when experiencing no progress. Two students reported that they tended to move to something new when they made no progress. For instance, Jacky viewed the difficulty in understanding a certain part of a course as a "hindrance". He observed,

This hindrance made me feel no progress and want to just let it go...I thought this thing wasn't suitable for me and I should do something 
else.(Jacky)

Jenny shared her similar learning experience. She tended to give up some tasks that she found difficult.

I didn't need to waste time on it, because I got stuck and made no progress.

I was going to do other things that were more interesting or fit for me.

(Jenny)

When they felt no progress, these participants tended to give up working on the tasks which required them to overcome obstacles. These accounts are consistent with a strong locomotion orientation.

Being focused on learning tasks. Three participants reported that they tended to be easily distracted when they worked on their learning-related tasks.

...you will learn for a while, but you will turn to your mobile phone, your microblog or watch a movie in the self-study room. Even in class, sometimes I was absent-minded while the teacher was lecturing. (Wu)

Della also said that she wanted to talk with others around when doing her assignments in the classroom. Locomotion is positively related to attention control when engaging in activities (Higgins et al., 2003). Thus, the tendency of being easily distracted is associated with a weak locomotion orientation.

In contrast, Ivy reported that she did not like to be interrupted when doing her work and wanted to remain highly focused till she completed the task. These data suggest that locomotion was more chronically accessible to Ivy than to the other two participants.

Alignment with below average promotion. In contrast with their below average levels of promotion, these participants reported tendencies associated with a strong promotion orientation. Specifically, they worked enthusiastically to achieve individual growth and aspirations, were open to change and new experiences, and were motivated by success and positive feedback.

Working to achieve individual aspirations and growth. Three participants reported promotion-related reasons that motivated them to study at university. For instance, Wu was working on the postgraduate entrance exam this year and was motivated by her dream to study in a top university. Similarly, Jenny observed that she wanted to attain her own dream rather than meet her parents' expectations. She also perceived studying for a master's degree as an opportunity for academic advancement.

I don't want to feel content with the status quo. A bachelor's degree isn't enough. I want to go on with a postgraduate programme to gain more 
knowledge and improve myself. (Jenny)

These accounts suggest that these students thought of studying at university as opportunities to achieve their dreams and growth, which characterizes promotion-related motivation.

Being motivated by success and positive feedback. All five participants reported that they were motivated by success.

Success gives me a sense of accomplishment and makes me happy and more motivated. (Jenny)

Besides being motivated by success, three students observed that they were motivated by positive feedback. For instance, Della said,

I feel happy when I get positive feedback from my teachers. I am more confident and work harder. (Della)

Importantly, four students stated that they felt demotivated when experiencing failure or receiving negative feedback. The negative emotions they experienced in these situations included being "very upset" (Ivy) and "lost and frustrated, a sense of incompetence" (Jenny). Similarly, Jacky mentioned that failure diminished his efforts and could even make him quit.

Failure dampened my enthusiasm for learning....If I failed repeatedly, I felt depressed. ... I might think this thing wasn't suitable for me and consider giving up. (Jacky)

These students' responses to success/failure and positive/negative feedback are consistent with a strong promotion orientation. Specifically, success and positive feedback encouraged them, but failure and negative feedback decreased their motivation.

Being enthusiastic and open to change. Jenny discussed her enthusiasm for reading beyond the course requirement in a literature course. This is a typical example of using eager strategies in learning settings (Molden \& Rosenzweig, 2016).

I also read after class many readings related to the texts in the book, which the teacher didn't ask us to read, but I would like to know more about those stuff. (Jenny)

Moreover, three participants reported that they were open to change and various opportunities for gains. For instance, Jacky wanted to pursue his master's degree abroad in the future, because he hoped to gain different educational and cultural experiences. Similarly, as a person from the north China, $\mathrm{Wu}$ wanted to study a postgraduate programme in a university in the south and experience a change of life. These reports reflect a strong promotion focus.

Alignment with below average prevention. The participants reported approaches to 
learning and learning-related tasks/activities that are consistent with both strong and weak prevention. In particular, they tried to meet their parental expectations, had prevention-related goals and were cautious with learning at university. Nevertheless, they also reported they tended to be careless with their assignments sometimes.

Meeting parental expectations and other requirements. Three participants reported that they viewed fulfilling parental expectations as their own responsibility. In particular, $\mathrm{Wu}$ mentioned that she was driven by living up to her parents' expectations.

My parents think that I have to apply for a master's degree. I think it is my duty not to let them down. Their expectations push me to make more efforts, but also put me under a lot of pressure. Sometimes I want to give up, but I dare not quit, because I have to meet their expectations. (Wu)

Ivy also reported her parents' expectations of her that she should study hard at university and take the postgraduate entrance exam and other exams for certificates. She said that it was her duty to follow their suggestions, because "they have invested a lot of money for my tutoring fees and I must try not to let them down".

As well as meeting their parental expectations, two students reported some other responsibilities they had to meet. For example, Ivy said that she worked hard to attain some professional certificates, because in a lot of companies these certificates were "the basic requirements for job seekers". These students were driven by their attempts to fulfill responsibilities, which characterizes prevention-related motivation.

Moreover, their prevention concerns were strong in the contexts of examinations. For instance, Jacky noted that he had to study mathematics courses even though he found them boring and difficult, because he "had to pass the final exams for credits". Wu also observed that she tried hard to work out difficult mathematics questions, because she "was afraid of failing in the final exam".

Being motivated by negative feedback and failure. Two participants reported that they were sensitive to negative feedback. In particular, Della said that she preferred the negative comments.

When someone points your problems out, you will realize that those problems deserve more attention and can make progress by correcting them. (Della)

Wu described a similar reaction to negative comments. She said that negative comments would help her identify her flaws and motivate her to do better next time. She also noted that she worked harder when she thought of the potential failure in the postgraduate 
entrance exam.

These accounts suggest that these participants paid particular attention to information about problems they needed to avoid. This vigilant strategy enabled them to prevent failure in the future. Again, these reports are consistent with a strong prevention focus.

Being cautious. All five participants reported that they were cautious with their studies at university. For example, in order not to fail in the final exam, they reviewed intensely weeks before the exams and followed the course requirements. As Wu said,

We got the prepared questions for the final exams. We could get satisfactory marks if we memorized those questions well. (Wu)

Notably, only some but not all of these participants worked carefully and tried to avoid mistakes. For instance, Ivy described herself as "a careful person". She read instructions very carefully when doing accounting exercises.

I read the instruction several times. First I read the instructions in general and then circled the key numbers and words and read them carefully to avoid making mistakes. (Ivy)

Jenny also mentioned that she was very cautious in exams. She checked her answers several times to identify spelling errors and incomplete answers.

In contrast, two students reported that they were careless when they worked on their assignments. For instance, Della described herself as a careless person and did not pay close attention to potential mistakes in her writings. These tendencies are associated with a weak prevention orientation.

Table 40 sums up the data reported by the five participants for each theme. The quantitative findings indicated that the Class 5 profile was characterized by below average and comparable levels of assessment, locomotion, promotion, and somewhat stronger prevention. As I discussed in this section, with the exception of promotion, the approaches to learning and learning-related tasks/activities reported by students classified in this class aligned with the characteristics of this motivation profile. 
Table 40

Summary of the Five Participants' Data for Each Theme (C5)

\begin{tabular}{|c|c|c|c|c|c|c|}
\hline \multirow[t]{2}{*}{ Theme } & \multirow[t]{2}{*}{ Category } & \multicolumn{5}{|c|}{ Participant } \\
\hline & & Della & Ivy & Jacky & Jenny & $\mathrm{Wu}$ \\
\hline \multirow[t]{3}{*}{$\begin{array}{l}\text { Alignment } \\
\text { with below } \\
\text { average } \\
\text { assessment }\end{array}$} & $\begin{array}{l}\text { Making critical } \\
\text { comparisons }\end{array}$ & $\begin{array}{l}\text { Compared } \\
\text { theories in some } \\
\text { courses }\end{array}$ & $\begin{array}{l}\text { Compared } \\
\text { theories in some } \\
\text { courses; } \\
\text { Compared herself } \\
\text { to classmates }\end{array}$ & $\begin{array}{l}\text { Compared } \\
\text { theories in some } \\
\text { courses }\end{array}$ & $\begin{array}{l}\text { Compared theories in } \\
\text { some courses; } \\
\text { Compared herself to } \\
\text { others }\end{array}$ & $\begin{array}{l}\text { Compared } \\
\text { theories in some } \\
\text { courses }\end{array}$ \\
\hline & $\begin{array}{l}\text { Reflecting on } \\
\text { previous } \\
\text { experiences }\end{array}$ & $\begin{array}{l}\text { Reflected on } \\
\text { previous } \\
\text { experiences }\end{array}$ & & & & $\begin{array}{l}\text { Reflected what } \\
\text { she had done } \\
\text { when reviewing }\end{array}$ \\
\hline & $\begin{array}{l}\text { Identifying } \\
\text { optimal goals }\end{array}$ & $\begin{array}{l}\text { Did not think } \\
\text { about long-term } \\
\text { goals }\end{array}$ & $\begin{array}{l}\text { Did not think } \\
\text { about long-term } \\
\text { goals }\end{array}$ & & Being aimless & \\
\hline \multirow{2}{*}{$\begin{array}{l}\text { Alignment } \\
\text { with below } \\
\text { average } \\
\text { locomotion }\end{array}$} & $\begin{array}{l}\text { Doing things step } \\
\text { by step }\end{array}$ & & $\begin{array}{l}\text { Worked out } \\
\text { questions step by } \\
\text { step }\end{array}$ & & $\begin{array}{l}\text { Followed steps in } \\
\text { writing essays }\end{array}$ & \\
\hline & $\begin{array}{l}\text { Doing something } \\
\text { new when } \\
\text { experiencing no } \\
\text { progress }\end{array}$ & & & $\begin{array}{l}\text { Did something } \\
\text { more interesting } \\
\text { or new when } \\
\text { making no } \\
\text { progress }\end{array}$ & $\begin{array}{l}\text { Gave up difficult } \\
\text { tasks when getting } \\
\text { stuck }\end{array}$ & \\
\hline
\end{tabular}




\begin{tabular}{|c|c|c|c|c|c|c|}
\hline \multirow[t]{2}{*}{ Theme } & \multirow[t]{2}{*}{ Category } & \multicolumn{5}{|c|}{ Participant } \\
\hline & & Della & Ivy & Jacky & Jenny & $\mathrm{Wu}$ \\
\hline \multirow{4}{*}{$\begin{array}{l}\text { Alignment } \\
\text { with below } \\
\text { average } \\
\text { promotion }\end{array}$} & $\begin{array}{l}\text { Being focused on } \\
\text { learning tasks }\end{array}$ & $\begin{array}{l}\text { Was easily } \\
\text { distracted }\end{array}$ & $\begin{array}{l}\text { Did not like } \\
\text { interruptions }\end{array}$ & & Was easily distracted & $\begin{array}{l}\text { Was easily } \\
\text { distracted }\end{array}$ \\
\hline & $\begin{array}{l}\text { Working to } \\
\text { achieve } \\
\text { individual } \\
\text { aspirations and } \\
\text { growth }\end{array}$ & \multirow{3}{*}{$\begin{array}{l}\text { Felt motivated by } \\
\text { success and } \\
\text { positive feedback }\end{array}$} & \multirow{3}{*}{$\begin{array}{l}\text { Felt motivated by } \\
\text { success and } \\
\text { positive feedback; } \\
\text { Felt demotivated } \\
\text { by failure and } \\
\text { negative feedback }\end{array}$} & $\begin{array}{l}\text { Studied to attain } \\
\text { individual growth }\end{array}$ & $\begin{array}{l}\text { Studied to attain } \\
\text { individual growth and } \\
\text { her dream }\end{array}$ & $\begin{array}{l}\text { Studied to attain } \\
\text { aspirations }\end{array}$ \\
\hline & $\begin{array}{l}\text { Being motivated } \\
\text { by success and } \\
\text { positive feedback }\end{array}$ & & & $\begin{array}{l}\text { Felt motivated by } \\
\text { success; } \\
\text { Felt demotivated } \\
\text { by failure }\end{array}$ & $\begin{array}{l}\text { Felt motivated by } \\
\text { success and positive } \\
\text { feedback; } \\
\text { Felt demotivated by } \\
\text { failure }\end{array}$ & $\begin{array}{l}\text { Felt motivated by } \\
\text { success }\end{array}$ \\
\hline & $\begin{array}{l}\text { Being } \\
\text { enthusiastic and } \\
\text { open to change }\end{array}$ & & & $\begin{array}{l}\text { Was open to new } \\
\text { experiences }\end{array}$ & $\begin{array}{l}\text { Read beyond the } \\
\text { course requirement }\end{array}$ & $\begin{array}{l}\text { Was open to } \\
\text { change }\end{array}$ \\
\hline $\begin{array}{l}\text { Alignment } \\
\text { with below } \\
\text { average } \\
\text { prevention }\end{array}$ & $\begin{array}{l}\text { Meeting parental } \\
\text { expectations and } \\
\text { other } \\
\text { requirements }\end{array}$ & $\begin{array}{l}\text { Lived up to } \\
\text { parental } \\
\text { expectations; } \\
\text { Set prevention- } \\
\text { related goals }\end{array}$ & $\begin{array}{l}\text { Lived up to } \\
\text { parental } \\
\text { expectations; } \\
\text { Met requirements; } \\
\text { Set prevention- } \\
\text { related goals }\end{array}$ & $\begin{array}{l}\text { Set prevention- } \\
\text { related goals }\end{array}$ & $\begin{array}{l}\text { Set prevention-related } \\
\text { goals }\end{array}$ & $\begin{array}{l}\text { Lived up to } \\
\text { parental } \\
\text { expectations; } \\
\text { Set prevention- } \\
\text { related goals }\end{array}$ \\
\hline
\end{tabular}




\begin{tabular}{|c|c|c|c|c|c|c|}
\hline \multirow[t]{2}{*}{ Theme } & \multirow[t]{2}{*}{ Category } & \multicolumn{5}{|c|}{ Participant } \\
\hline & & Della & Ivy & Jacky & Jenny & $\mathrm{Wu}$ \\
\hline & $\begin{array}{l}\text { Being motivated } \\
\text { by negative } \\
\text { feedback }\end{array}$ & $\begin{array}{l}\text { Was sensitive to } \\
\text { negative feedback }\end{array}$ & & & & $\begin{array}{l}\text { Was sensitive to } \\
\text { negative } \\
\text { feedback; } \\
\text { Was motivated by } \\
\text { avoiding potential } \\
\text { failure }\end{array}$ \\
\hline & Being cautious & $\begin{array}{l}\text { Was careless; } \\
\text { Reviewed key } \\
\text { points for exams }\end{array}$ & $\begin{array}{l}\text { Reviewed key } \\
\text { points for exams; } \\
\text { Reading } \\
\text { instructions } \\
\text { carefully }\end{array}$ & $\begin{array}{l}\text { Reviewed key } \\
\text { points for exams }\end{array}$ & $\begin{array}{l}\text { Reviewed key points } \\
\text { for exams; } \\
\text { Answered and } \\
\text { checked test questions } \\
\text { carefully }\end{array}$ & $\begin{array}{l}\text { Reviewed key } \\
\text { points for exams; } \\
\text { Was not very } \\
\text { careful when } \\
\text { doing assignments }\end{array}$ \\
\hline
\end{tabular}




\section{Chapter Summary}

Within this chapter, I reported qualitative findings from the second phase of the study. Interview participants who exhibited a common motivation profile were regarded as a case. Findings from each case suggested that the approaches to learning or learning-related tasks/activities reported by the interview participants generally aligned with the characteristics of the motivation profiles they displayed. However, the interviewees recruited from $\mathrm{C} 3$ and $\mathrm{C} 4$ also reported tendencies that were not in line with the characteristics of their motivation profiles. In the next chapter, I will integrate the quantitative and qualitative findings to discuss the characteristics of each of the five motivation profiles uncovered. In addition, I will highlight the important contributions the study makes. 


\section{Chapter Six Discussion}

Drawing on the relevant literature, I discuss the quantitative and qualitative findings through the combined theoretical lenses of the regulatory focus theory (Higgins, 1997, 2012) and the regulatory mode theory (Kruglanski et al., 2000). The quantitative and qualitative findings are integrated to capture the features of the motivation profiles identified in this research and gain important insights into the complex interrelationships among the key motivation orientations (i.e., assessment, locomotion, promotion, and prevention) that shape motivation and self-regulation in learning at university.

Within this chapter, I first analyze the characteristics of each of the five motivation profiles. Then, I evaluate commonalities and differences among the motivation profiles that share similar elements with regard to grit, theories of intelligence, critical thinking, effort regulation, and evaluations/perceptions of academic success. Subsequently, I discuss similarities/differences in motivational tendencies/manifestations among the interview participants who exhibited the same motivation profile. Finally, I highlight the important and novel findings that advance the existing literature on motivation and motivation effects.

\section{Characterization of the C1 Profile}

The latent profile analysis revealed that the motivation profile underlying $\mathrm{C} 1$ was characterized by high/very high levels on all four motivation orientations. In addition, assessment, locomotion, and promotion were comparable in strength, whereas prevention was the strongest of all four orientations. The approaches to learning and learning-related tasks/activities reported by the interview participants recruited from $\mathrm{C} 1$ were largely consistent with the characteristics of their motivation profile.

Characteristics of the $\mathbf{C 1}$ motivation profile. The approaches to learning and learning-related tasks/activities reported by the interview participants recruited from $\mathrm{C} 1$ are consistent with their strong assessment orientations. Specifically, they reported their efforts to make sense of their experiences at university in different ways. In addition, all participants reflected on their past failure-related experiences and attempted to identify the optimal ways 
of performing their learning tasks. These participants also reported engaging in critical comparisons. In particular, they evaluated the particular knowledge acquired in a course and this process helped them understand what they learned. They also compared themselves with their classmates and friends with regard to academic performance. Consistent with the regulatory mode theory (Higgins, 2012; Higgins et al., 2003), this self-evaluation made them experience negative emotions. Moreover, these students expended time and effort to evaluate alternative goals in decision-making processes and goal pursuits. Due to their concerns regarding a thorough consideration of options, they tended to be indecisive. Nevertheless, their careful assessment of goals and ways to achieve goals may also play an adaptive role, that is, to constrain their inclination to initiate any action, which was associated with strong locomotion.

The approaches to learning reported by these participants were also consistent with their strong locomotion orientations. In particular, they reported preference for performing quickly and smoothly in learning-related tasks or activities. For example, they discussed that following step-by-step instructions and engaging in their tasks in an organized way, which fit their strong locomotion orientation, helped them to make continuous progress. This finding is consistent with the regulatory fit theory (Higgins, 2012). When people experience regulatory fit, they have strengthened task engagement and are more motivated to pursue their goals (Higgins, 2012). In addition, these participants reported being enthusiastic to strive for new goals after attaining a previous goal. They also tended to switch to other goals to facilitate steady progress. Importantly, for these participants, strong locomotion mitigated some of the drawbacks associated with strong assessment (e.g., procrastination). For instance, the students reported that they tended to start working on their assignments immediately after receiving the tasks. In line with the regulatory mode theory (Higgins, 2012; Kruglanski et al., 2000), strong locomotion put a limit on assessing too much, and thus, motivated them to take action and perform their tasks quickly rather than wait and contemplate how to do the assignments.

Consistent with the combination of strong promotion and strong prevention that characterizes $\mathrm{C} 1$, these students were motivated by both achieving hopes and aspirations and 
by fulfilling duties and responsibilities. For example, these participants reported that they regarded their goals (e.g., getting admitted to a postgraduate programme) as an opportunity to attain their aspirations and academic advancement. Driven by these ideals, they were optimistic and open to change and new experiences. These tendencies are consistent with their strong promotion orientations. At the same time, they worked hard to fulfil their obligations to family and meet parental expectations and adopted prevention-related goals (e.g., not failing in the examinations). Due to their strong prevention focus, they also employed vigilant strategies such as carefully following the course requirements and checking repeatedly what they had done to avoid mistakes. Similarly, these participants' responses to positive and negative feedback were also consistent with their dual strong promotion-prevention focus. Their strong promotion orientations made them motivated by positive feedback, which contributed to their increased eagerness for advancement. Meanwhile, their strong prevention orientations made them pay attention to negative feedback regarding their mistakes and the strategies that they needed to prevent failure in future. Emotional experiences with regard to success/failure reported by one participant are also consistent with these students' dual strong promotion-prevention focus. Specifically, in line with her strong promotion orientation, the participant felt happy when she attained success, and was thus motivated to devote extra effort for achieving more success. Additionally, consistent with her strong prevention orientation, she reported experiencing agitation-related emotions after failure, which consequently appeared to strengthen her vigilance to avoid failure in future. This example illustrates how promotion and prevention worked together, which made the participant likely to work harder after success and be resilient in the face of failure.

Inter-profile differences. In this section, I compare motivation effects associated with the motivation profile underlying $\mathrm{C} 1$ to the $\mathrm{C} 3$ and $\mathrm{C} 2$ profiles respectively. As I discuss below, these comparisons reveal important aspects regarding how the four motivation orientations interacted to shape motivation effects.

C1 and C3. The C1 and C3 profiles had similar and high levels of assessment, locomotion, and promotion. However, the quantitative data indicated that prevention was 
very strong in participants in $\mathrm{C} 1$ and very weak in $\mathrm{C} 3$. These two profiles were associated with significant differences in some motivation factors. Specifically, with regard to both consistency of interest and perseverance of effort (i.e., the two dimensions of grit), $\mathrm{C} 1 \mathrm{had}$ significantly higher average levels than $\mathrm{C} 3$. Considering that these two profiles differed mainly in the absolute magnitude of prevention, variability in the strength of prevention was related to significant differences in grit. These findings suggest that for the individuals classified in $\mathrm{C} 1$, high levels of prevention, which is concerned with the fulfilment of responsibilities, could counterbalance some of the negative tendencies (e.g., abandoning important goals when facing difficulties), which were associated with strong promotion and locomotion, and motivated them to be more committed to their goal pursuits and work harder to overcome difficulties (i.e., to be more gritty).

In contrast, for individuals in $\mathrm{C} 3$, the prevention orientation would be too weak to contribute to the self-regulation, let alone to keep in check the strong promotion and strong locomotion. As a result, when facing difficulties, these students were unlikely to be very gritty in goal pursuits because their strong locomotion and strong promotion motivated them to give up the original goals and select other goals that could better facilitate steady progress and advancement.

The mean level of self-reported critical thinking was significantly lower for $\mathrm{C} 1$ than C3; this difference was small. This finding suggests that when assessment, locomotion and promotion were all strong, high levels of prevention were not associated with high levels of critical thinking. This finding was not in the direction of the hypothesis proposed by Miele and Wigfield (2014), which states that prevention is likely to be positively associated with critical thinking.

In contrast, the average score of Cognitive Reflection Test (CRT) was higher for C1 than for C3, although this difference was not statistically significant. This finding was in the direction hypothesized by Miele and Wigfield (2014). In addition, there were no significant differences between $\mathrm{C} 1$ and $\mathrm{C} 3$ with regards to incremental and entity theories of intelligence, effort regulation, and perceptions/evaluations of academic success. These findings suggest that when assessment, locomotion, and promotion were comparable and of 
strong magnitude, large differences in the strength of prevention did not lead to significant differences in these motivation factors.

C1 and $\mathbf{C 2}$. The $\mathrm{C} 1$ and $\mathrm{C} 2$ profiles had a different pattern of similarity and difference with regard to the magnitude of regulatory focus and mode orientations. These two profiles had virtually identical and very high levels of prevention, but had medium differences in assessment, locomotion, and promotion. In addition, in both $\mathrm{C} 1$ and $\mathrm{C} 2$, assessment, locomotion, and promotion were comparable in strength.

These two profiles were also associated with significant differences in some motivation factors. With respect to consistency of interest, perseverance of effort, self-reported critical thinking, and effort regulation, $\mathrm{C} 1$ was associated with significantly larger average values than C2. These findings suggest that when prevention was virtually equal at high levels, medium differences in assessment, locomotion, and promotion were associated with variability in these motivation effects. Interestingly, given that prevention was almost identical in $\mathrm{C} 1$ and $\mathrm{C} 2$, it is also apparent that high (vs. medium) levels of assessment, locomotion, and promotion were linked to stronger levels of grit when prevention was constant and high. Particularly, locomotion is positively associated with the tendency to attend to a task and persist until completing a task (Kruglanski et al., 2000). Having higher levels of locomotion, the participants classified in $\mathrm{C} 1$ were more likely than their counterparts from $\mathrm{C} 2$ to be willing to overcome difficulties and devote effort to the tasks at hand, and thus displayed higher levels of grit and effort regulation. In addition, having stronger assessment, they were more likely to engage in thoroughly comparing and evaluating the knowledge they learned. This may be why these participants had higher levels of self-reported critical thinking than those classified in $\mathrm{C} 2$.

$\mathrm{C} 1$ had a significantly lower average value of the incremental theory of intelligence than $\mathrm{C}$; ; this difference between the two classes was very small. There were no significant differences between $\mathrm{C} 1$ and $\mathrm{C} 2$ with respect to the entity theory of intelligence. The average score of CRT was higher for C2 than for C1. However, these differences were not statistically significant. Moreover, there were no statistically significant differences between these two profiles with regard to perceptions/evaluations of academic success. Overall, these findings suggest that when prevention was virtually identical at high levels, medium differences in the 
strengths of assessment, locomotion, and promotion were associated with small/insignificant differences with regard to these motivation factors.

Individual differences within C1. Although the qualitative data suggest that the aspects reported by the interview participants were generally consistent with the motivation profile underlying $\mathrm{C} 1$, the interviewees shared some (but not all) characteristics or tendencies associated with the $\mathrm{C} 1$ profile. The motivation profiles identified in the quantitative phase are collective profiles for the participants classified in the same class and consist of average values of the motivation orientations for the given latent class. Therefore, it is possible that the interview participants display some different manifestations of the motivation orientations. Specifically, all four participants described their use of critical comparison and evaluations. In contrast, only two participants reported using the strategy of weighing up different options in order to make the correct decisions. These data suggest that the former strategy might have been a more common manifestation of assessment than the latter for participants exhibiting the $\mathrm{C} 1$ profile. Moreover, all four participants reported that they preferred to do things in a step-by-step manner, were eager to pursue new goals when they achieved a given goal, and tended to do their assignments immediately. These aspects were common manifestations of their high levels of locomotion. However, only two participants recalled that they tended to switch goals when they could not see progress in their goal pursuits. This suggests the latter characteristic could have been a relatively less common manifestation of strong locomotion. Further, the participants reported different experiences with regard to success and failure. Two participants reported that they were motivated by success, but demotivated by failure, which could suggest that they had stronger promotion than prevention. One participant reported that he was more motivated by failure than success, which could suggest that his prevention was stronger than his promotion. In contrast, one participant reported that she was motivated by both success and failure, which is consistent with the dual strong promotion and prevention focus that characterizes $\mathrm{C} 1$. 


\section{Characterization of the C2 Profile}

The latent profile analysis indicated that the motivation profile associated with $\mathrm{C} 2$ was characterized by comparable and slightly below average levels of assessment, locomotion, and promotion and very high levels of prevention. For this class, prevention dominated in strength the other motivation orientations. The approaches to learning and learning-related tasks/activities reported by the interview participants recruited from C2 were generally consistent with the characteristics of their motivation profile.

Characteristics of the $\mathbf{C 2}$ motivation profile. The approaches to learning and learning-related tasks/activities reported by the participants recruited from $\mathrm{C} 2$ revealed that they displayed characteristics associated with both strong and weak assessment orientations. Specifically, participants reported that they tended to compare themselves with other people. In line with the regulatory mode theory (Higgins, 2012; Higgins et al., 2003), these selfevaluation processes involved high standards for personal performance and, thus, made them vulnerable to negative emotions. Moreover, these interviewees employed strategies that prioritized accuracy when they worked on the learning-related tasks/activities. For instance, they reported that they preferred to check the format of their papers repeatedly to ensure the accuracy and sought for accurate information about what they found ambiguous in class. These strategies were consistent with a strong assessment (Higgins, 2012; Kruglanski et al., 2000).

Furthermore, they reported that they evaluated different options in decision-making and tried to identify the course of action they found most appropriate. In line with the regulatory mode theory (Higgins, 2012; Kruglanski et al., 2000), they tended to be indecisive due to this thorough evaluation of alternatives. In addition, they noted that they engaged in counterfactual thinking (i.e., reflecting on what would have happened if they had done it differently) and felt regretful. Both of these tendencies are consistent with strong assessment, which motivated them to evaluate previous decisions so that they could make better decisions in the future (Molden, 2012). Importantly, these participants also reported tendencies that are typically associated with a weak assessment orientation. For example, they discussed not 
engaging in critical evaluation of the particular knowledge that they learned in their courses. Instead, they accepted and memorized what they were taught without questioning.

Similarly, the approaches to learning and learning-related tasks/activities reported by these interviewees also indicate that they showed tendencies associated with both strong and weak locomotion orientations. Specifically, they reported that when they worked on their assignments, they preferred to maintain continuous progress by addressing each of the requirements step by step. This tendency characterizes a strong locomotion orientation (Higgins, 2012; Kruglanski et al., 2000). However, they noted that they were not very keen to strive for new goals when they had achieved a previous goal. This tendency to stop for some time before moving along entailed interrupting steady movement and, thus, corresponds to a weak locomotion orientation (Higgins, 2012; Kruglanski et al., 2000). In addition, they found it difficult to fully concentrate on the learning-related tasks. Locomotion is positively related to having control over one's attention when engaging in tasks (Higgins et al., 2003). Thus, these interviewees' propensity of being distractible was consistent with a weak locomotion orientation. Considering these results together with the corresponding qualitative findings pertaining to assessment, it is apparent that these interviewees reported more strategies that fit a strong assessment orientation than strategies that are associated with strong locomotion. Therefore, it can be concluded that the motivational effects (or manifestations) corresponding to assessment were somewhat more prevalent than their counterparts pertaining to locomotion, despite the fact that these two orientations were of comparable strength in this class.

Further, this group of participants reported few examples of approaches to learning or learning-related tasks/activities that were likely to be related to a promotion focus. In particular, interview data from two participant suggested that they had promotion-related motivation for learning at the university. Specifically, they perceived their goals (e.g., pursuing a postgraduate programme) as an opportunity to improve their academic or career development. However, they did not report any eager strategies to attain these ideal goals. These findings suggest that the impact of their promotion orientations was less apparent than the influences of assessment and locomotion orientations, despite the fact that assessment, 
locomotion, and promotion were comparable in strength in C2. Notably, for these participants, their below average promotion was likely to be constrained by their very high levels of prevention.

Lastly, the approaches to learning and learning-related tasks/activities reported by the interviewees from $\mathrm{C} 2$ were consistent with the very high levels of prevention that characterized their motivation profile. These students reported that they were motivated by meeting obligations and responsibilities. Specifically, they regarded working hard at university as an important responsibility to fulfil their parents' expectations. Apart from their focus on obligations to family, these respondents also reported other duties that they thought they had to fulfil, such as meeting the task/course requirements. Moreover, they adopted prevention-related goals (e.g., not failing in the examinations and securing a postgraduate programme). Consistent with their strong prevention, these participants studied in a vigilant way to protect against anything that would prevent them from fulfilling these responsibility. For instance, they followed carefully the course requirements and focused mainly on the particular knowledge that would be tested in the final exams. Moreover, when they worked on their assignments, these students started earlier than the deadlines and did not procrastinate. On one hand, it was likely that the tendency to start early increased their promptness to initiate action, which is a characteristic of both prevention and locomotion. On the other hand, this tendency was likely to inhibit their assessment, which is positively associated with procrastination (Pierro et al., 2011). Although they started early on their assignments, they worked slowly by checking carefully all the things that might go wrong.

To achieve their prevention goals, these participants appeared to use strategies that contributed to the enhancement of their assessment orientations. For example, they assessed cautiously pros and cons of different options so that they could identify the most appropriate ways to attain their prevention goals and prevent failure. This example showed how prevention and assessment interacted to shape individuals' strategies during their goal pursuits. Furthermore, emotional experiences with regard to success/failure reported by these participants were also consistent with their strong prevention focus. Specifically, for these participants, success reduced the vigilance that they needed to prevent negative outcomes in 
the future and, therefore, decreased their motivation to engage in their learning-related tasks/activities. In contrast, failure strengthened their vigilance and, thus, motivated them to work harder. Finally, these participants' responses to negative feedback were also consistent with a strong prevention focus. In line with the regulatory focus theory (Higgins, 2012), they paid close attention to critical feedback that identified the areas in most need of attention, so that they could minimize risks of making errors.

Inter-profile differences. In this section, I compare motivation effects related to the C2 and C5 profiles. As I discuss below, these comparisons will show how the interplay among the four motivation orientations shaped motivation effects and self-regulation.

For the $\mathrm{C} 2$ and $\mathrm{C} 5$ profiles, assessment, locomotion, and promotion were virtually indistinguishable; in contrast, prevention was very strong in $\mathrm{C} 2$ and of medium magnitude in C5. There was no statistically significant difference with regard to consistency of interest between these two profiles. Considering that these profiles differed mainly in the absolute magnitude of prevention, variability in the prevention strength was not associated with a difference in consistency of interest, when the remaining three orientations were of similar below average magnitude. Similarly, there was no statistically significant difference between C2 and C5 in terms of perseverance of effort. This finding suggests that when assessment, locomotion, and promotion were comparable and of below average magnitude, a medium difference in the strength of prevention was not associated with a difference in perseverance of effort. Taking these findings together, a medium difference in the magnitude of prevention is not expected to be related to a difference in grit when assessment, locomotion, and promotion are virtually equal at average levels. Considering these findings together with the corresponding results highlighted when comparing $\mathrm{C} 1$ and $\mathrm{C} 3$, it is apparent that medium differences in prevention were not associated with differences in grit when assessment, locomotion, and promotion were all of average strength. In contrast, high differences in prevention were associated with differences in grit when assessment, locomotion, and promotion were all strong. These results suggest that no motivation orientations can function in isolation; instead, the effect of each orientation relies on the strength of the other orientations (Higgins, 2012). 
There were no statistically significant differences between C2 and C5 with respect to both incremental and entity theories of intelligence. These results suggest that when assessment, locomotion, and promotion were similar (at average levels), a medium difference in the strength of prevention did not translate into a significant difference with regard to any of the two theories of intelligence. These results are similar to those pertaining to the corresponding comparison between $\mathrm{C} 1$ and $\mathrm{C} 3$. Taken together, these findings suggest that when assessment, locomotion, and promotion are comparable, regardless of whether they are of high or average magnitude, large or medium differences in the strength of prevention did not lead to differences in any of the two theories of intelligence.

Again, similar to the case of differences noted with regards to self-reported critical thinking between $\mathrm{C} 1$ and $\mathrm{C} 3$, the average value of self-reported critical thinking was significantly larger for students classified in C5 than for their counterparts in C2. This finding was not consistent with the hypothesis proposed by Miele and Wigfield (2014) that prevention is positively related to critical thinking. In contrast, there were no statistically significant differences with regard to CRT between C2 and C5. However, the average score of CRT was larger for C2 than for C5. This finding, although not statistically significant, was in the direction hypothesized by Miele and Wigfield (2014).

With respect to effort regulation, there were statistically significant differences between C2 and C5. Specifically, the average level of effort regulation was significantly higher for students classified in C5 than for their counterparts in C2. This finding suggests that a medium difference in the magnitude of prevention was associated with a difference in effort regulation when the other three orientations were identical and of average magnitudes. Specifically, higher levels of prevention were associated with lower levels of effort regulation. A plausible explanation of this finding is that in the $\mathrm{C} 2$ profile prevention dominated in strength the other orientations. In turn, the dominance of prevention may be associated with maladaptive tendencies, which could inhibit people's goal strivings (Cornwell et al., 2015). For instance, participants having the $C 2$ profile were likely to feel extremely anxious when they engaged in very difficult tasks, and, therefore, gave up effort. This is a possible reason why these individuals displayed low levels of effort regulation. Taking this finding together with the corresponding results from comparing $\mathrm{C} 1$ and $\mathrm{C} 3$, it follows that 
prevention is likely to be related to effort regulation only when assessment, locomotion, and promotion are not simultaneously strong.

The average value of perceptions/evaluations of academic success was found to be significantly larger for students classified in $\mathrm{C} 2$ than for their counterparts in C5. This finding was in line with the results reported by Rosenzweig and Miele (2016) that prevention-focused university students, when given adequate time to complete the tests, scored higher on the academic tests than did their counterparts who had strong promotion orientations.

Considering this finding together with the corresponding results from comparing $\mathrm{C} 1$ and $\mathrm{C} 3$, it follows that a higher mean level of prevention contributed more to the difference in perceptions/evaluations of academic success when the other three orientations were of average strength than when they were all of high magnitude. This conclusion is similar to the one enunciated above with regards to effort regulation. Again, these findings provide support for Higgins' (2012) theorizing that the role and impact of each motivation tendency depend on the strength of the other tendencies.

Individual similarities/differences within C2. The interviewees recruited from C2 shared some of the tendencies associated with their collective motivation profile. Specifically, consistent with their below average assessment, none of the four participants compared critically or evaluated the particular knowledge they learned in their courses. In addition, consistent with their below average locomotion, they all tended to take a break before striving for a new goal (when they attained a previous goal). Moreover, all four participants reported that they were motivated by upholding their duties and responsibilities, had prevention-related goals, employed vigilant strategies in learning at university, and were sensitive to failure and negative feedback. These aspects appeared to be common manifestations of the given motivation orientations for participants exhibiting the $\mathrm{C} 2$ profile.

However, there were individual variations among these interviewees with regard to the characteristics or tendencies associated with the C2 profile. Specifically, two participants discussed that they tended to compare themselves with their classmates and friends; three participants reported using strategies that prioritized accuracy; two participants noted their tendency to weigh up alternatives in decision-making. Moreover, with regard to locomotion, two participants reported that they preferred to work on their assignments in a step-by-step 
manner and three participants recalled that it was difficult to maintain their attention during task engagement. In addition, only two participants described their promotion-related motivation at university. These qualitative findings suggest that these tendencies or propensities were less common manifestations of the given motivation orientations for participants exhibiting the $\mathrm{C} 2$ profile. Overall, in line with the strong prevention characterizing $\mathrm{C} 2$, the approaches to learning and learning-related tasks/activities that were most consistently reported by all participants recruited from $\mathrm{C} 2$ were those associated with a prevention focus.

\section{Characterization of the C3 Profile}

The latent profile analysis showed that the $\mathrm{C} 3$ motivation profile was characterized by comparable and high/very high mean levels of assessment, locomotion, and promotion, but very low mean levels of prevention. The approaches to learning and learning-related tasks/activities reported by the interview participants recruited from C3 were generally consistent with the characteristics of their motivation profile except for some aspects related to prevention.

Characteristics of the $\mathbf{C 3}$ motivation profile. The approaches to learning and learning-related tasks/activities reported by the participants recruited from $\mathrm{C} 3$ were consistent with their high mean levels of assessment. Specifically, the interview data reported by these participants suggested that they engaged in critical comparison and evaluation processes. For instance, they reported that they evaluated different theories or assertions they learned in their courses. These evaluations helped them acquire a clear understanding of the particular knowledge and identify the ideas they found convincing. They also tended to compare themselves with their classmates and friends. Consistent with the regulatory mode theory (Higgins, 2012; Higgins et al., 2003), they had more accurate self-evaluations, but were prone to negative emotions.

Moreover, these participants were inclined to devote extra time to evaluate potential goals and effective means to attain goals before initiating action. Similar to the case of their counterparts classified in $\mathrm{C} 1$, their strong assessment tendencies may inhibit their 
locomotion-related tendencies to initiate movement or change. Notably, two participants reported that they tended to procrastinate when they received assignments. Considering that assessment is positively related to procrastination (Pierro et al., 2011), it was likely that assessment was more chronically accessible to these participants (compared to locomotion, which is negatively associated with procrastination). Furthermore, consistent with their strong assessment, these participants reported that they drew on their previous failure- related experiences and identified the areas that needed improving.

In addition, one participant reported that careful assessment of goals enabled her to identify several important goals that she could pursue at the same time. This strong assessment tendency could counterbalance some of the maladaptive consequences associated with having strong locomotion and promotion that characterized this profile. Specifically, individuals having a strong locomotion orientation tend to advance toward several goals at the same time and may suffer negative affect (e.g., frustration) for performing only one task at a time (Pierro et al., 2013). This preference for multi-tasking, in combination with strong promotion concerns with maximizing gains, makes it likely that individuals classified in $\mathrm{C} 3$ experience stronger negative emotions when they focus on one goal rather than pursuing multiple goals. However, as these individuals have very high levels of assessment, they are likely to select a set of worthwhile goals that could be pursued simultaneously. In turn, this approach reduces chances of experiencing negative emotions.

The approaches to learning and learning-related tasks/activities reported by these participants were also consistent with their strong locomotion. Specifically, interviewees reported that they liked to follow a sequence of steps when working on their assignments and attempted to maintain steady progress through tasks. In addition, when they experienced little or no progress, these students tended to switch to other goals so that they could facilitate movement or change (Kruglanski et al., 2000). The interviewees also reported that they were fully focused on the task at hand till they completed it. As discussed previously, locomotion is positively related to attentional control during task engagement (Higgins et al., 2003). Therefore, their tendency to focus on tasks till their completion is consistent with a strong locomotion orientation. In addition, the participants discussed that they preferred to use 
strategies that prioritized speed over accuracy. For instance, they tried to finish their writing tasks within a short period of time and did not pay close attention to spelling or grammar. Once again, these strategies are consistent with a strong locomotion orientation. Finally, one participant noted that he was enthusiastic to get started when he received an assignment. As I discussed in the case of the interviewees recruited from $\mathrm{C} 1$, strong locomotion may counterbalance the negative tendency to procrastinate, which is positively associated with high levels of assessment (Pierro et al., 2011).

Consistent with the high level of promotion that characterized the $\mathrm{C} 3$ profile, these participants reported that they were motivated to attain aspirations and individual growth. Specifically, these respondents thought of being educated at university as an opportunity to realize their dreams and seek academic or career advancement. In addition, they reported their enthusiasm when they pursued their ideal goals. For instance, these students held an optimistic outlook about their future, which encouraged them to overcome difficulties in the pursuits of their ideal goals. They read beyond the course requirement and sought help from classmates and teachers; these strategies are typical examples of eager means (Molden \& Rosenzweig, 2016). These participants also used strategies that prioritized speed (e.g., reading passages quickly in English tests). Consistent with their strong promotion orientations, they thought that these strategies could maximize their opportunities to gain higher scores in the limited time (Higgins, 2012; Molden, 2012). Similarly, two interviewees reported emotional experiences regarding success/failure and positive/negative feedback that were also consistent with a strong promotion focus. In line with the regulatory focus theory (Higgins, 2012), they paid particular attention to, and were motivated by, success and positive feedback, which fuelled their enthusiasm to work harder. In contrast, they reported that they felt upset when they faced failure or received negative feedback; failure/negative feedback reduced their keenness to devote extra effort in their learning-related tasks/activities.

Finally, although the quantitative results revealed that the $\mathrm{C} 3$ profile was characterized by very weak prevention, the approaches to learning and learning-related tasks/activities reported by these participants suggested that they displayed some characteristics associated 
with both weak and strong prevention orientations. Specifically, two participants reported that they tended to be careless and paid little attention to mistakes they were likely to make when working on the assignments. This tendency is consistent with a weak prevention focus. Notably, all interviewees reported having prevention-related goals. Specifically, they thought that it was an important responsibility not to fail in the exams. In line with the regulatory focus theory (Higgins, 2012; Molden \& Rosenzweig, 2016), although these respondent have had strong promotion orientations, (and, thus, may have perceived most goals in terms of aspirations and advancement), their prevention focus could be temporarily activated (e.g., in the context of academic examinations). Consistent with their temporarily strong prevention orientations, these students paid particular attention to areas that were in most need of improvement, so that they could prevent failure in their courses. Finally, emotional experiences with regard to success/failure and positive/negative feedback reported by one participant are also consistent with a strong prevention focus. Specifically, this participant was sensitive to, and motivated to devote extra effort by, failure and negative feedback.

Individual similarities/differences within C3. Similar to the cases of the participants classified in $\mathrm{C} 1$ and $\mathrm{C} 2$, the interviewees recruited from $\mathrm{C} 3$ shared some characteristics or tendencies associated with their collective $\mathrm{C} 3$ profile and displayed some different manifestations of their motivation orientations. Specifically, all three participants reported that they engaged in critical comparisons and evaluations. In addition, two participants reported thinking carefully about the value of goals and the means to achieve their goals before taking action. Moreover, two participants discussed that they reflected on their previous experiences. These findings suggest that engagement in critical comparisons/evaluations appeared to be a somewhat more common manifestation of strong assessment than the other strategies (i.e., evaluating goals and means to achieve goals, reflecting on one's experiences) for participants exhibiting the $\mathrm{C} 3$ profile.

All three participants reported that they tended to abandon their goals and switch to other goals when they saw little or no progress. This aspect was a common manifestation of their strong locomotion. In addition, two participants noted that they preferred to do things in a step-by-step manner; two participants tended to maintain their attention during task 
engagement; two participants reported using strategies that prioritized speed; one participant tended to be keen to initiate action. These findings suggest that the latter tendencies (i.e., working in a step-by step manner, focusing on tasks at hand, prioritizing speed, and taking action quickly) were relatively less common manifestations of strong locomotion for participants from C3 (compared to goal switching/abandoning).

All three participants reported the motivation to achieve ideal goals and their enthusiasm to pursue these goals. These aspects are common manifestations of their strong promotion focus. In addition, two participants reported that they were motivated by success, but demotivated by failure, which was consistent with the strong promotion that characterized their motivation profile. In contrast, one participant reported that he was more motivated by failure and negative feedback. Moreover, he also reported that he preferred to check his work carefully to avoid making mistakes, whilst the other two interviewees tended to be relatively careless. These results suggest that his prevention was stronger than that of the other participants. Finally, all three participants reported prevention-related goals and vigilance in the pursuits of these goals. This finding suggests that for these interviewees, prevention was temporarily primed in the context of examinations, despite the fact that their collective motivation profile was characterized by very weak prevention.

\section{Characterization of the C4 Profile}

The latent profile analysis showed that the $\mathrm{C} 4$ motivation profile was characterized by low/very low levels of assessment, locomotion and promotion and below average levels of prevention. In this class, prevention was much stronger than any of the other motivation orientations. Moreover, assessment was stronger than both locomotion and promotion, which were of comparable strength. The approaches to learning and learning-related tasks/activities reported by the interview participants recruited from $\mathrm{C} 4$ suggest both consistency and inconsistency with the characteristics of their motivation profile. I discuss these aspect below.

Characteristics of the $\mathbf{C} 4$ motivation profile. Despite the fact that the $\mathrm{C} 4$ profile was characterized by weak assessment, the approaches to learning and learning-related tasks/activities reported by the participants recruited from $\mathrm{C} 4$ suggest that they exhibited 
some characteristics associated with both strong and weak assessment orientations.

Specifically, they tended to compare themselves with other people. In line with the tenets of the regulatory mode theory, this self-evaluation concern made them vulnerable to negative emotions (Higgins, 2012; Higgins et al., 2003). In addition, these students noted that they reflected on their previous experiences and felt remorse over their decisions or behaviours. Both of these two tendencies are consistent with a strong assessment orientation. Notably, these participants also reported characteristics that are typically related to a weak assessment orientation. Specifically, they discussed that they did not evaluate critically the particular knowledge they learned in their courses. Instead, these respondents attempted to memorize what they were taught in class. Moreover, one participant reported that during her first two years at university, she rarely expended time and effort to (i) identify optimal goals she could pursue and (ii) seek means she could use to achieve the goals.

The approaches to learning and learning-related tasks/activities reported by these interviewees are consistent with the very low level of locomotion that characterized their motivation profile. Specifically, the three participants reported that they found it difficult to maintain their attention when they worked on their learning-related tasks. As discussed previously, this propensity of being distractible is consistent with a weak locomotion orientation (Higgins et al., 2003). In addition, two participants reported that they tended to procrastinate and were not keen to get started when they received an assignment; locomotion is negatively related to procrastination (Higgins, 2012; Higgins et al., 2003; Pierro et al., 2011). Moreover, the students' reported lack of motivation to initiate action is also consistent with a weak locomotion orientation.

Considering these findings together with the corresponding qualitative results pertaining to assessment, it follows that these interviewees reported some strategies that fit a strong assessment orientation, but no strategies that are associated with strong locomotion. Therefore, it can be concluded that the motivational effects of assessment were somewhat more prevalent than their counterparts corresponding to locomotion; this conclusion is consistent with the fact that assessment was stronger than locomotion in this class. These results suggest that, for these participants, locomotion was too weak to play its role in self- 
regulation, let alone to compensate for the strength of assessment. This conclusion is in line with Higgins' (2012) theorizing that a motivation orientation needs to have a certain strength to be effective to contribute to self-regulation. As assessment was stronger than locomotion in this profile, it is likely that these participants ruminated about what they should do (or what they should have done), and then felt remorse for not doing it, rather than take action to initiate any change.

Although the quantitative results revealed that the $\mathrm{C} 4$ profile was characterized by a very low level of promotion, these interviewees described a few examples of approaches to learning and learning-related tasks/activities that were likely to be related to a strong promotion focus. In particular, two participants reported promotion-related motivation for learning at university. Specifically, they perceived their goals (e.g., studying English or pursuing a postgraduate programme) as an opportunity to achieve their dreams and improve their career development. In addition, these two participants reported that they were motivated by success and demotivated by failure. These reactions to success and failure are consistent with a strong promotion orientation.

These findings suggest that promotion goals were likely to be more chronically accessible to these participants than prevention goals. However, these interviewees did not report using any eager strategies to achieve their ideal goals. Taking these results together with the corresponding qualitative findings pertaining to $\mathrm{C} 1, \mathrm{C} 2$ and $\mathrm{C} 3$, it follows that differentiation between high vs. below average vs. low levels on a motivation orientation may be reflected better in the repertoire of strategies these individuals reported using than in the types of goals they adopted. In particular, participants having high levels on a given motivation orientation generally reported employing a greater range of strategies that were consistent with the high levels on this specific motivation orientation than their counterparts having low or below average levels on this orientation.

Lastly, the approaches to learning and learning-related tasks/activities reported by the interviewees recruited from C4 suggest that these participants displayed characteristics related to both strong and weak prevention orientations. In line with strong prevention, these participants were motivated to fulfil duties and obligations. For example, they regarded 
studying at university as an important responsibility to live up to parental expectations. The interview data also suggested that they were motivated to maintain safety and security (e.g., securing a satisfactory job after graduation and maintaining academic ranking in the final exams). Moreover, they set prevention goals (e.g., trying not to fail in the examinations and securing a postgraduate programme).

Motivated by their prevention concerns, these participants followed carefully the course requirements and focused only on the key points in their courses that would be tested in the final exams. In addition, one participant reported that she started to work on her assignment on time and checked carefully her assignments to avoid mistakes. She also noted that she paid particular attention to failure and negative feedback, which motivated her to expend extra effort. These tendencies are consistent with a strong prevention focus. However, the other participants recruited from $\mathrm{C} 4$ reported that they were demotivated by failure and tended to be relatively careless (e.g., these students did not pay close attention to the mistakes they were likely to make when they worked on their assignments). In addition, one participant reported that she rarely cared about maintaining academic rankings during her first two years at university. These characteristics are consistent with a weak prevention orientation. Taking these findings together with the corresponding qualitative results regarding promotion, it is apparent that the interviewees selected from $\mathrm{C} 4$ reported more strategies that fit a strong prevention focus than strategies that are related to a strong promotion focus. Therefore, it can be concluded that the motivational effects (or manifestations) pertaining to prevention were somewhat more prevalent than their counterparts with regard to promotion. This finding is consistent with the fact that prevention was much stronger than promotion in this profile.

Inter-profile differences. In this section, motivation effects related to the C4 and C5 profiles are compared. As I discuss in the following, these comparisons reveal how the four motivation orientations interacted to shape motivation effects and self-regulation.

For the $\mathrm{C} 4$ and $\mathrm{C} 5$ profiles, prevention was virtually identical (at below average levels). However, assessment, locomotion, and promotion were of weak/very weak magnitudes in $\mathrm{C} 4$ and of medium magnitudes in $\mathrm{C} 5$. Moreover, prevention was much 
stronger than the other motivation orientations in $\mathrm{C} 4$, whilst the four orientations were of comparable levels in C5. These two profiles were associated with significant differences in some motivation effects. Specifically, C5 had significantly larger average levels than C4 with regard to consistency of interest, perseverance of effort, self-reported critical thinking, and effort regulation. These findings suggest that when prevention was virtually equal at average levels, medium differences in assessment, locomotion, and promotion were associated with variability in these motivation effects. Considering these findings together with the corresponding results from comparing $\mathrm{C} 1$ and $\mathrm{C} 2$, it follows that when prevention is comparable, regardless of whether it is of high or average magnitude, differences in the strength of assessment, locomotion, and promotion were associated with differences in these motivation factors. Importantly, consistent with Higgins' (2012) conceptualization of motivation, these motivation effects were generally more adaptive when assessment, locomotion, and promotion were stronger (vs. weaker).

C4 had a significantly larger average value of the incremental theory of intelligence than C5; this difference between the two classes was small. The average value of entity theory of intelligence was higher for $\mathrm{C} 4$ than for $\mathrm{C} 5$; however, this difference was not statistically significant. These findings suggest that when prevention was virtually identical at average levels, medium differences in the strengths of assessment, locomotion, and promotion were associated with small/insignificant differences with regard to the two theories of intelligence. These result are similar to those pertaining to the corresponding comparison between $\mathrm{C} 1$ and $\mathrm{C} 2$. Taken together, these findings suggest that when prevention was virtually identical, regardless of whether it was of high or average magnitude, medium differences in the strengths of assessment, locomotion, and promotion were associated with small/insignificant differences with regard to the theories of intelligence.

The average score of Cognitive Reflection Test was larger for C5 than for C4. C5 had a higher average value regarding perceptions/evaluations of academic success than $\mathrm{C} 4$. However, these differences were not statistically significant. These findings suggest that when prevention was virtually identical at average levels, medium differences in the strengths of assessment, locomotion, and promotion did not translate in significant differences with respect to these outcomes. 
Individual similarities/differences within C4. The interview participants recruited from $\mathrm{C} 4$ shared some of the characteristics associated with their collective motivation profile. Specifically, consistent with the weak assessment that characterized the $\mathrm{C} 4$ profile, the three participants did not critically evaluate the particular knowledge they learned in their courses. Consistent with their weak locomotion, they found it difficult to focus during task engagement. In addition, two participants noted that they tended to procrastinate and lack the drive to take action; these tendencies are in line with a weak locomotion orientation. Moreover, the three participants reported that they had set prevention goals and adopted vigilant strategies during engagement in learning-related tasks/activities. These tendencies appeared to be common manifestations of the given motivation orientations for participants exhibiting the $\mathrm{C} 4$ profile.

However, the qualitative findings suggest that there were individual difference among these interviewees with regard to the characteristics associated with the $\mathrm{C} 4$ profile. Specifically, with regard to assessment, two participants reported that they compared themselves with other people; two participants reported reflecting on previous experiences; one respondent did not tend to think carefully about her goals and how to achieve these goals. Further, with regard to promotion, two participants reported setting some ideal goals at university and noted that they were motivated more by success than by failure. Finally, in terms of prevention, two participants reported that they were motivated to meet duties; one participant reported that she tried to avoid making mistakes during task engagement and was motivated by failure and negative feedback. In contrast, two participants stated that they were demotivated by failure and careless with their assignments.

Importantly, the qualitative data reported by one interviewee suggest that the motivation effects associated with prevention were much more prevalent for her than for the other participants recruited from this class. Specifically, this interviewee consistently reported tendencies that were typically associated with a strong prevention focus. For example, she was motivated to meet parental expectations and maintain security, paid particular attention to failure and negative feedback, preferred to start early, and checked her work carefully. The quantitative results revealed that the probability with which this participant was classified in C4 was 0.51 , and the probability with which she was classified in C2 was 0.485 . Her 
assignment to classes was borderline; therefore, it was likely that she displayed some characteristics associated with the $\mathrm{C} 2$ profile, which was characterized by very strong prevention. This case sheds important light on how the findings of the qualitative analysis complemented the results of the quantitative analysis. In particular, it suggests that when researchers evaluate the alignment between a participant's motivational manifestations and her/his motivation profile, they need to take into account the specific probabilities of the given individual being classified into each latent class.

\section{Characterization of the C5 Profile}

The latent profile analysis indicated that the motivation profile underlying C5 was characterized by comparable assessment, locomotion, and promotion as well as somewhat stronger prevention. In this class, all four motivation orientations were at below average levels. The approaches to learning and learning-related tasks/activities reported by the interview participants recruited from C5 were generally consistent with the characteristics of their motivation profile except for some aspects related to promotion.

Characteristics of the $\mathbf{C 5}$ motivation profile. The approaches to learning and learning-related tasks/activities reported by the interview participants recruited from C5 suggest that these participants showed tendencies related to both strong and weak assessment orientations. Specifically, these students reported that they tended to make critical comparisons between themselves and their classmates and friends. These self-evaluations, which characterize a strong assessment orientation, made them prone to negative emotions (Higgins, 2012; Higgins et al., 2003). In addition, these interviewees discussed that they tended to reflect on what they had done in the past. This tendency, which is consistent with strong assessment, motivated them to evaluate previous experiences so that they could identify the most appropriate course of action they would take in the future (Higgins, 2012). Moreover, these participants reported that they sometimes evaluated the particular knowledge they learned in some of their courses. Notably, these respondents also discussed that sometimes they memorized what they were taught without critical evaluation. They also stated that they seldom tried to identify the optimal goals that could be pursued at university. 
Both of these latter tendencies (i.e., not consistently engaging in critical evaluations, rarely thinking about best goals/means to achieve goals) are typically associated with a weak assessment orientation.

The approaches to learning and learning-related tasks/activities reported by these interviewees also reveal that they displayed characteristics associated with both strong and weak locomotion orientations. Specifically, these participants reported that they preferred to follow a sequence of steps when they worked on their learning-related tasks. Furthermore, these respondents noted that they tended to give up their original goals when they felt little or no progress in the goal pursuits and made efforts for new goals. Both of these tendencies characterize a strong locomotion orientation (Higgins, 2012; Kruglanski et al., 2000). However, these interviewees also reported that they were prone to distractions and not focused on the task at hand. As I discussed in the cases of $\mathrm{C} 2$ and $\mathrm{C} 4$, this propensity of being easily distracted is consistent with a weak locomotion orientation. Notably, two participants reported that they started to work immediately after receiving their assignments, while the other participants tended to procrastinate. Considering that locomotion is negatively related to procrastination (Pierro et al., 2011), this finding suggests that it was likely that locomotion was more chronically accessible to the former two participants than to the latter ones.

Although the C5 motivation profile was characterized by a below average level of promotion, the approaches to learning or learning-related tasks/activities reported by this group of participants suggest that they displayed some characteristics related to a strong promotion focus. In particular, these participants noted that they perceived their goals (e.g., striving for a career as a teacher and pursuing a postgraduate programme) as an opportunity to achieve their dreams and improve their career development. Consistent with these promotion concerns, these respondents reported using some eager strategies to pursue their ideal goals. For instance, they were open to change and new experiences, read with enthusiasm beyond the course requirement, and tried not to miss opportunities for career development. In addition, these interviewees reported that they felt happy at success or positive feedback, and, thus, were motivated to work harder. Three interviewees also 
mentioned that they were demotivated by failure and negative feedback. These emotional experiences are consistent with having a strong promotion focus.

Finally, the approaches to learning and learning-related tasks/activities reported by the interviewees from $\mathrm{C} 5$ indicate that they showed characteristics associated with both strong and weak prevention orientations. Specifically, these participants reported that they were motivated to live up to their parents' expectations and meet the course requirements. Moreover, they reported having prevention goals. For instance, all participants tried to prevent failure in the final examinations and secure a place in a postgraduate programme. Consistent with strong prevention, these participants reported that they studied in a vigilant way to prevent failure. For example, they focused mainly on the particular knowledge that would be tested in the final exams and reviewed it carefully before the examinations. Two participants noted that they read the instructions carefully and checked repeatedly to avoid potential mistakes when working on the assignments or during the examinations. In addition, emotional experiences with regard to failure and negative feedback reported by two participants were also consistent with a strong prevention focus. Specifically, for these participants, failure and negative feedback highlighted information about their mistakes or weakness that they needed to prevent in the future, and, thus, strengthened their motivation to work harder. This finding suggests that prevention was more chronically accessible to these interviewees than promotion. In contrast, three interviewees noted that they tended to be relatively careless when they worked on the assignments. This tendency is consistent with a weak prevention focus.

Individual similarities/differences within C5. The interviewees recruited from C5 shared some of the tendencies associated with their collective motivation profile. Specifically, consistent with their below average assessment, the five participants sometimes critically evaluated the particular knowledge they learned in their courses, but sometimes memorized the key points without questioning. Moreover, with regard to promotion, the five participants reported that success and positive feedback motivated them to be more engaged in learning. Lastly, these interviewees all reported adopting prevention goals and using vigilant strategies when they worked on their learning-related tasks/activities. These 
tendencies appeared to be common manifestations of the given motivation orientations for participants exhibiting the $\mathrm{C} 5$ profile.

However, there were individual variations among these interviewees with regard to the characteristics or tendencies associated with the C5 profile. Specifically, with respect to assessment, two participants noted that they tended to compare themselves with other people; two participants reported their tendency to reflect on previous experiences. In contrast, three participants discussed that they did not think carefully about optimal goals they could strive for at university. With regard to locomotion, two participants reported that they preferred to maintain continuous movement by following steps when they worked on their assignments; two participants recalled their tendency of goal switching. In contrast, three interviewees noted that they were likely to be distracted during goal pursuits. With regard to promotion, three participants discussed their ideal goals at university and their enthusiasm to pursue these goals. In contrast, the other interviewees selected from C5 did not report setting any ideal goals and using eager strategies. Finally, with regard to prevention, three interviewees reported their motivation to meet parental expectations; two interviewees noted that they were motivated by failure and negative feedback; two participants stated that they were careful when they worked on assignments. In contrast, three participants mentioned that they were demotivated by failure and negative feedback and tended to be careless with learningrelated tasks.

\section{Contributions and Implications of the Findings}

This research advances the existing literature on motivation in important ways. I discuss the key contributions of this research and highlight the implications of the findings in the following sections. First, I delineate similarities and differences among the motivation profiles identified in this study and their counterparts uncovered in previous research and discuss their theoretical and practical implications. Then, I argue that the findings from the study support Higgins' (2012) theorizing that motivation and motivation effects are shaped by the interrelationships among individuals' strivings for value, truth, and control effectiveness. In addition, I illustrate important trade-offs among promotion, prevention, assessment, and 
locomotion and how these trade-offs shape motivation effects. I also discuss motivational manifestations/characteristics associated with different strengths of a given motivation orientation. Finally, I evaluate the extent to which different motivation profiles are associated with distinct levels of motivation factors/outcomes.

Similarity and variability with regard to motivation profiles. In this research, five distinct motivation profiles were identified, which accounted for the variability of the interrelationships among promotion, prevention, locomotion, and assessment for a sample of Chinese undergraduate students. Similarly, recent studies, using samples of New Zealand secondary school students (Harring \& Hodis, 2016; Hodis et al., 2017), also uncovered a set of motivation profiles anchored by the four motivation orientations. Although the samples in my research and those in Harring \& Hodis (2016) and Hodis et al. (2017) were recruited from different cultural contexts and had different developmental ages, four structurally similar motivation profiles were consistently identified (see details in the section of "Results of LPA" in the chapter of Quantitative Findings). Specifically, the motivation profiles corresponding to $\mathrm{C} 1$ in my research and C6 in Harring and Hodis (2016) and Hodis et al. (2017) were both characterized by high/very high mean levels of assessment, locomotion, promotion, and prevention. The $\mathrm{C} 3$ motivation profile in my research was similar in shape to the $\mathrm{C} 4$ profile in the other set of studies; both profiles were characterized by strong assessment, locomotion, and promotion, but very weak prevention. The motivation profiles corresponding to $\mathrm{C} 4 \mathrm{in} \mathrm{my}$ research and C2 in Harring \& Hodis (2016) and Hodis et al. (2017) were both characterized by low/very low levels of assessment, locomotion, promotion, and prevention. The motivation profile associated with C5 in my research and that underlying C1 in Harring \& Hodis (2016) and Hodis et al. (2017) were both characterized by below average and comparable levels on all four motivation orientations.

These consistently identified motivation profiles provide empirical evidence for Higgins' (2012) contention regarding the universality of the significant motivational states produced by the regulatory focus and regulatory mode orientations. Higgins $(2008,2012)$ stated that promotion, prevention, locomotion, and assessment are universal psychological mechanisms and are "basic to all self-regulation" (Higgins, 2008, p. 616). Due to their survival value, every individual in every culture endorses each of the four motivation 
orientations, although to different degrees (Higgins, 2012). Importantly, these motivation orientations "significantly influence the ways in which people organize their experiences and the nature of strategies, construal, affect, and motivation in goal pursuit" (Dweck, Higgins, \& Grant-Pillow, 2003, pp. 241-242).

Higgins (2012) conceptualized a variety of motivation patterns characterized by distinct combinations of the four motivation orientations (e.g., strong vs. weak promotion combined with strong vs. weak prevention, combined with strong vs. weak locomotion, and with strong vs. weak assessment). These structurally similar profiles identified across three independent large samples from two culturally and developmentally distinct populations capture some of "the significant motivational states that vary across persons, situations, organizations, and cultures" (Higgins 2012, p. 314). Hence, these findings provide important information that advances knowledge of motivation processes and attendant effects.

There were some differences among the structurally similar profiles in the two sets of studies (my research, on the one hand, Harring \& Hodis (2016) and Hodis et al. (2017), on the other hand). As noted before, the motivation profiles identified in my research (which collected data from a sample of Chinese undergraduate students) encompassed relatively higher levels of assessment, somewhat lower levels of promotion, and much higher levels of prevention than the profiles uncovered in the samples of New Zealand secondary school students (see details in the section of "Results of LPA" in the chapter of Quantitative Findings).

These differences are in line with the previous findings of cross-cultural studies (Higgins, 2008; Higgins et al., 2008). These studies reported that individuals from Asian countries (e.g., Japan) had more predominant prevention and assessment orientations and a weaker promotion orientation compared to their counterparts from western countries (e.g., the US and Italy; Higgins, 2008; Higgins et al., 2008). Higgins (2012) postulated that cultural environments and values play significant roles in shaping the variability in the predominance of promotion, prevention, locomotion and assessment across individuals and cultures. For example, in cultural contexts where fulfilling responsibilities and interdependent selfconstrual (i.e., defining oneself in terms of social roles and connectedness to others; Markus \& Kitayama, 1991) are stressed (e.g., in Asian cultures), individuals tend to have predominant 
prevention and assessment orientations (Higgins, 2012; Molden \& Miele, 2008; Molden \& Rosenzweig, 2016). In contrast, in cultural contexts, where personal aspirations and independent self-construal (i.e., perceiving oneself as independent and unique from others; Markus \& Kitayama, 1991) are highlighted (e.g., in western cultures), individuals are more likely to have predominant promotion and locomotion orientations (Higgins, 2012; Molden \& Miele, 2008; Molden \& Rosenzweig, 2016).

Identifying structurally similar motivation profiles in the two distinct populations, which were different with regard to culture/developmental age, has important theoretical implications. One implication is that particular configurations of the associations among the motivation orientations may be universal and can be observed in different cultural contexts. For example, the C3 profile in my research and the C4 profile in Hodis et al. (2017) had very similar motivation structures. Both profiles included high levels of assessment, locomotion, and promotion, but very low levels of prevention. Moreover, these two profiles had the strongest assessment and the weakest prevention of all profiles identified in each respective sample. As participants from mainland China and New Zealand in these two sets of studies would be considered as subgroups in eastern and western cultures respectively, it is likely that this configuration of the motivation orientations can be identified in other countries (e.g., Japan and U.S.) as well.

Second, researchers argue that age may have an effect on individuals' development of regulatory focus and mode orientations (Higgins, 2012; Kooij et al., 2014; Liu \& Yao, 2018; Pennington \& Roese, 2003). Wigfield et al. (2015) posited that it is likely that motivation orientations do not "stabilize until late adolescence, when children's self-regulatory and selfevaluation capacities have matured" (p. 15). In line with this argument, the consistently identified motivation profiles in the two developmentally distinct populations imply that the development of motivation orientations may stabilize in adolescence and early adulthood.

Liu and Yao (2018) identified four distinct profiles defined by promotion and prevention orientations for a sample of Chinese preadolescents and adolescents. These motivation profiles were similar in the relative magnitude of promotion and prevention to four profiles uncovered in my research. Specifically, the C1 profile in Liu and Yao (2018) and the $\mathrm{C} 1$ profile in my research were both characterized by high levels of promotion and 
prevention. The C2 profile in Liu and Yao (2018) and the C5 profiles in this research both included moderate levels of promotion and prevention. The C3 profile in Liu and Yao (2018) and the $\mathrm{C} 4$ profile in my research were both characterized by weak promotion and weak prevention. The C4 profile in Liu and Yao (2018) and the C3 profile in my research both encompassed strong promotion and weak prevention. Taking these results together with the corresponding findings in Harring \& Hodis (2016) and Hodis et al. (2017), it follows that structurally similar motivation profiles can be identified in the two developmentally distinct populations.

In addition, these findings have significant implications for education practice. Understanding important individual variations in motivation profiles will help teachers/educators identify subgroups of students. For example, students exhibiting a motivation outlook similar to $\mathrm{C} 4$ identified in this study are likely to be at risk for lack of motivation and engagement in learning. This information will enable teachers to model students having distinct motivation profiles different strategies and provide appropriate incentives and feedback (i.e., that match optimally their motivation profiles). For instance, for students having the $\mathrm{C} 3$ profile (i.e., having strong/very strong promotion, locomotion, and assessment, but very weak prevention), teachers may design the learning environment in ways that highlight gains and offer positive feedback. This could create regulatory fit, and, thus, increase students' task engagement and performance in learning settings (Higgins, 2012; Molden \& Rosenzweig, 2016).

Supporting Higgins' conceptualization of motivation. Another key contribution of this research is that the findings provide empirical evidence for Higgins' (2012) conceptualization that motivation encompasses strivings for value, truth, and control effectiveness. As presented previously, the five distinct motivation profiles that were identified in this research mapped the complex interrelationships among promotion, prevention, locomotion, and assessment; taken together, these motivation orientations measure the three types of effectiveness strivings proposed by Higgins (2012).

An investigation of the structures of the five profiles showed that within each profile each orientation had distinct absolute and relative magnitudes. For example, within the C3 profile, assessment, locomotion, and promotion were strong, but prevention was very weak. 
Prevention was much weaker than the other three orientations, which were all of comparable levels. In addition, in all profiles except the $\mathrm{C} 2$ profile, promotion/prevention, which measured strivings for value effectiveness, did not dominate in strength assessment and locomotion, which measured truth and control effectiveness, respectively. In line with Higgins' (2012) theoretical propositions, these findings suggest that motivation comprises all three types of effectiveness strivings. These findings are also consistent with the results reported by Harring and Hodis (2016) and Hodis et al. (2017). These authors revealed that the motivation orientations differed in magnitude within and across the motivation profiles identified in their studies; in none of the profiles, promotion and prevention (that measure strivings for value effectiveness) dwarfed in strength locomotion and assessment, which pertain to control and truth effectiveness, respectively.

The results reported in this study also provide important information on the interrelationships among the three types of effectiveness strivings and their conjoint influences on individuals' behaviors, cognition and emotions (Higgins, 2012). Higgins (2012) argued that to understand motivation and motivation effects, it is necessary to take into consideration value, truth, and control effectiveness as well as the interactions among them. This is the case because the three types of strivings for effectiveness interact with one another to shape self-regulation, goal setting and goal pursuits (Higgins, 2012; Higgins et al., 2008). As discussed previously, inter-profile comparisons in this research revealed that there were significant differences among some of the profiles in terms of motivation factors/outcomes that play important roles in learning settings (e.g., grit, critical thinking, theories of intelligence, effort regulation, and perceptions/evaluations of academic success); some of these differences were characterized by large/very large effect sizes. Notably, profiles that had some similar elements (e.g., had comparable levels on one or more orientations) were associated with some significant differences in motivation factors. For example, mean levels of assessment, locomotion, and promotion in $\mathrm{C} 1$ were similar to their counterparts in $\mathrm{C} 3$; however, these two profiles differed considerably in the strength of prevention. Differences between these two profiles were statistically significant only with regard to some of the motivation constructs investigated (i.e., grit and self-reported critical thinking).

In a similar manner, the studies of Harring and Hodis (2016) and Hodis et al. (2017) 
also indicated that distinct motivation profiles were associated with significant differences in key motivation constructs (i.e., expectancies of success, a sense of accomplishment related to learning something new, striving to advance knowledge, and adopting the goal to achieve academic ambitions); some of these differences were also characterized by (very) large effect sizes. Particularly, the motivation profiles that had low and very low levels of locomotion and promotion were linked to lower mean levels on these outcomes than their counterparts in which these orientations were of strong magnitude.

Importantly, the influence of each type of effectiveness depends on the strength of the other strivings (Higgins, 2012). For example, for the motivation profiles associated with C2 and C5, assessment, locomotion, and promotion were virtually identical, but prevention was very strong in $\mathrm{C} 2$ and of below average levels in $\mathrm{C} 5$. The quantitative findings revealed that this medium-sized difference with regard to prevention was not associated with statistically significant differences in the two facets of grit (i.e., consistency of interest and perseverance of effort). In contrast, the $\mathrm{C} 1$ and $\mathrm{C} 3$ profiles had comparable and strong assessment, locomotion, and promotion; however, prevention in $\mathrm{C} 1$ was much stronger than in $\mathrm{C} 3$. The quantitative findings showed that $\mathrm{C} 1$ was associated with significantly higher levels of grit than C3. These findings suggest that medium-sized differences in prevention were not associated with significant differences in grit when assessment, locomotion, and promotion were all of medium magnitude, whilst very large differences in prevention led to significant differences in grit when assessment, locomotion, and promotion were all strong. Therefore, interactions among assessment and locomotion (pertaining to truth and control related effectiveness, respectively) set constraints on the influence that prevention alone (i.e., one facet of value effectiveness) could have on motivation effects. Hence, these findings provide clear evidence that the three effective strivings interact (i.e., work together) to shape both the types and the magnitudes of attendant motivation effects (Harring \& Hodis, 2016; Hodis et al., 2017).

Findings about similarities/differences among motivation profiles with regard to key outcomes examined in this study are generally in line with Higgins' (2012) theorizing about how the three ways of being effective interact to shape motivation effects. Take the $\mathrm{C} 4$ profile as an example. This profile was characterized by having low/very low levels of assessment, 
locomotion, and promotion as well as below average levels of prevention. Consistent with the regulatory focus theory (Higgins, 1997, 2012) and the regulatory mode theory (Higgins et al., 2003; Kruglanski et al., 2000), individuals exhibiting a motivation outlook similar to the C4 profile may have weak/very weak truth, control, and value effectiveness. Specifically, these individuals may not be motivated to make sense of their learning experiences at university, take action to facilitate progress, and value any desired outcomes (Higgins, 2012). Therefore, it is likely that they are at risk for lacking motivation and disengaging from learning (Hodis, 2018a; Hodis et al., 2017).

Quantitative findings from this study are generally in line with these hypotheses. Results revealed that the motivation profile underlying $\mathrm{C} 4$ was associated with significantly lower mean levels on most of the criteria examined in this study than any of the other profiles. Specifically, participants exhibiting this motivation profile had the lowest mean levels of consistency of interest, perseverance of effort (i.e., the two facets of grit), and effort regulation. In addition, they had the second lowest mean levels of self-reported critical thinking and perceptions/evaluations of academic achievement. Notably, the quantitative results in this research also indicated that students having the $\mathrm{C} 4$ profile had higher mean levels of incremental and entity theories of intelligence than their counterparts exhibiting the other motivation profiles; however, these differences were often small or not statistically significant.

Likewise, as discussed above, Hodis and his colleagues (2017), who collected data from a population of secondary school students from New Zealand, identified a motivation profile that was structurally similar to the $\mathrm{C} 4$ profile. These authors found that this profile was associated with the lowest mean levels of sense of accomplishment, strivings to advance knowledge, and the goal to attain academic ambitions as well as the second lowest average value of expectancies of success among all motivation profiles identified in their studies.

The results from this research suggest important theoretical implications. Specifically, this study suggests that the complex interrelationships among effective strivings for value, control and truth can be mapped by distinct motivation profiles defined by the regulatory focus and mode orientations. Findings from this research also suggest that in goal pursuits, these three types of effectiveness function concurrently (i.e., work together) as critical 
determinants of motivation and motivation effects. These results imply that conceptualization of motivation should include not only value effectiveness, but also control and truth effectiveness.

\section{Highlighting the important motivational trade-offs among the motivation}

orientations. Each motivation orientation has "trade-offs that produce benefits under some conditions and costs under other conditions" (Higgins, 2012, p. 398). If a motivation profile includes two or more strong motivation orientations, their conjoint influences on motivation effects are likely to be associated with important trade-offs among them (Higgins, 2012; Higgins et al., 2003; Kruglanski et al., 2000). Particularly, the tendencies associated with the strong orientation(s) are likely to constrain some of the effects that the other orientation(s) alone would have on motivation (Cornwell et al., 2015; Higgins, 2012; Molden \& Miele, 2008). Examinations of the five motivation profiles identified in this research highlight the important motivational trade-offs among promotion, prevention, locomotion and assessment, and how these trade-offs shape motivation and self-regulation. I discuss these trades-off below.

When both strong promotion and prevention orientations are activated in an individual, the two orientations can interact effectively, each keeping in check the negative propensities of the other (Higgins, 2012, 2014). For example, a strong promotion focus is associated with adaptive tendencies to be sensitive to, and be motivated by, positive feedback and success (Higgins, 2012; Lockwood et al., 2002; Lockwood, Sadler, Fyman, \& Tuck, 2004; Shu \& Lam, 2011, 2016). These tendencies have some potential downside effects on motivation and self-regulation. In particular, when they face negative feedback/failure, promotion-focused individuals are likely to ignore critical feedback that helps identify areas in need of attention; they are also likely to experience sadness that might reduce their enthusiasm to strive for their original goals (Higgins, 2012; Scholer \& Higgins, 2012).

Strong prevention is likely to counterbalance some negative tendencies related to strong promotion. Specifically, high levels of prevention make individuals experience agitation-related emotions when they fail or receive negative feedback (Higgins, 2012; Scholer \& Higgins, 2012). This negative affect strengthens their vigilance. Consequently, they could pay particular attention to negative feedback and failure-related information so 
that they could avoid making mistakes in the future (Higgins, 2012; Lockwood et al., 2002, 2004).

Strong prevention may also engender some maladaptive tendencies. For example, prevention-focused individuals tend to be content with maintaining a current (barely) satisfactory condition. Thus, they are likely to maintain suboptimal states, even though they would be able to make progress. In this case, strong promotion may be more adaptive (Scholer \& Higgins, 2012). Qualitative findings in this research provide some illustrative examples of these trade-offs. For instance, the interview participants exhibiting the $\mathrm{C} 1$ profile, which was characterized by dual strong promotion and prevention orientations, noted being motivated to devote more effort to learning-related tasks/activities by both positive and negative feedback; one participant reported working harder after success and being resilient after failure. However, these findings are not consistent with the results reported by Shu and Lam (2011). In their study, these authors found that students having strong promotion orientations were more motivated by success feedback (e.g., they wrote more correct words and were more persistent in the word association test). In contrast, those who had strong prevention orientations were more motivated by failure feedback than their promotionfocused counterparts. Notably, their study did not find that the participants having a combination of strong promotion and strong prevention were motivated by both success and failure-related feedback. Nevertheless, it is important to note that the qualitative findings from this study were based on the interviewees' self-reports, rather than by evaluating their actual motivational outcomes as Shu and Lim (2011) did. Thus, it is possible that some participants provided responses on the basis of what they knew they ought to do, which may be different from what they actually did.

Other important quantitative findings in this study are that participants exhibiting the $\mathrm{C} 1$ profile had the highest mean levels of consistency of interest and perseverance of effort, and, thus, had the highest mean levels of grit among all profiles. Moreover, the differences between $\mathrm{C} 1$ and the other classes with regard to the two criteria were statistically significant and had moderate to large effect sizes. These findings also shed light on how the trade-offs between promotion and prevention shape motivation. Specifically, promotion relates positively to expectancy of success, whilst prevention is not significantly related to this 
construct (Hodis, 2018b; Hodis \& Hodis, 2015). Therefore, when individuals having a strong promotion focus face difficulties and perceive that there is a low likelihood of success, they are likely to give up the original important goals and switch to new goals (Molden et al., 2008). In this scenario, a strong prevention orientation could constrain individuals' tendency to abandon their important goals and could motivate them to be more committed to the original goal pursuit and persevere to overcome difficulties (i.e., to be more gritty). Importantly, this constraining process was unlikely to be at play in some of the motivation profiles, especially in the $\mathrm{C} 3$ profile, where prevention was very weak and dwarfed in magnitude by promotion. Indeed, the quantitative results in this research showed that participants having the $\mathrm{C} 3$ profile had relatively low mean levels of consistency of interest and perseverance of efforts, and, thus, were not very gritty.

In addition, findings in this research shed light on the key trade-offs between locomotion and assessment. On the one hand, strong locomotion could counterbalance some of the negative tendencies related to strong assessment (e.g., being lost in thinking; Cornwell et al., 2015; Higgins, 2012). One important finding of this study is that the interview participants recruited from $\mathrm{C} 1$ and $\mathrm{C} 3$, both of which exhibited strong locomotion and strong assessment, tended to start working immediately after they received an assignment. In this case, high levels of locomotion put a limit on assessing too much, and thus, motivated them to begin to work quickly on their tasks. This result is in line with findings indicating that locomotion is negatively associated with procrastination and motivates individuals to initiate timely action (Higgins, 2012; Higgins et al., 2003; Pierro et al., 2011). This tendency, in turn, mitigates procrastination, which is positively related to strong assessment (Pierro et al., 2011).

On the other hand, strong assessment could constrain some of the drawbacks of strong locomotion (i.e., effecting change without direction; Cornwell et al., 2015; Higgins, 2012). The interview participants recruited from $\mathrm{C} 1$ and $\mathrm{C} 3$ noted that they tended to have a thorough evaluations of different options before they made important decisions; these evaluation involved thinking of the value and attainability of the goals as well as identifying ways to achieve their important goals. For these students, high levels of assessment set a limit on their inclination to start any action, which was associated with strong locomotion. Moreover, strong assessment enabled one interviewee recruited from C3 to find a set of 
valuable goals that she could pursue at the same time. This tendency could counterbalance frustration high locomotors are likely to experience when they have to work towards a single goal at a time (Pierro et al., 2013). Again, these counterbalancing processes involving locomotion and assessment were unlikely to play an important role in some of the motivation profiles, where one or both of these orientations were very weak/relatively weak (e.g., C2, $\mathrm{C} 4$, and C5).

Within the motivation profiles that involved high levels of promotion and/or prevention and locomotion (e.g., C1 and C3), strong promotion/prevention is likely to be effective at compensating for some of the self-regulatory drawbacks associated with strong locomotion (e.g., tendency to effect change without paying much attention to gains or losses; Cornwell et al., 2015). Specifically, a strong promotion focus could motivate strong locomotors to make continuous progress towards attaining ideal goals or gains; a strong prevention focus could motivate these individuals to avoid mistakes while maintaining movement. Some examples from the qualitative results in this study illustrated this process. For instance, the interview participants selected from C3 reported that they preferred to work on the learning tasks (e.g., in reading comprehension tests) at a fast pace; at the same time, they tried to gain higher scores in the tests. Another example was provided by the interviewees from $\mathrm{C} 1$. They noted that they began to work on an assignment when they received it. They also tended to check their work carefully in order to avoid mistakes.

In turn, strong locomotion could prompt self-regulatory tendencies that can counterbalance negative tendencies associated with a strong promotion focus. For instance, the quantitative findings revealed that participants classified in C3 had the highest mean level of effort regulation. This result illustrates a potential trade-off between locomotion and promotion. Specifically, locomotion is positively associated with persisting and maintaining focus during task engagement (Higgins et al., 2003). This propensity is likely to offset the tendency to give up important goal pursuits when facing difficulties, which is related to strong promotion. In this case, these participants from C3 were likely to maintain efforts till they completed a task even if they encountered difficulties.

Taken together, investigations of the motivation profiles enabled identifying important motivational trade-offs that shaped key cognitive, behavioral, and affective aspects of goal 
pursuits. These findings support Higgins' (2012) theorizing and other substantive studies, which showed that having two or more strong orientations interacting effectively could produce desirable outcomes (Higgins, 2012; Kruglanski et al., 2000; Molden \& Rosenzweig, 2016). This is the case because strong orientations both support and constrain one another (as discussed above), to maximize the self-regulatory benefits and minimize the potential downsides associated with each orientation (Higgins, 2012; Molden \& Rosenzweig, 2016).

These findings suggest an important practical implication for learning environments. For optimal outcomes in learning setting, it is important for students to be aware of the potential motivational trade-offs and regulate their motivation effectively, that is, to identify and then "maintain the level and type of motivation needed to optimally pursue some goal" (Miele \& Scholer, 2018, p. 3). Specifically, students may identify motivational deficits (e.g., having one or more weak motivation orientations) and implement regulation strategies to enhance the given motivation orientation. For example, as discussed above, students having a motivation outlook similar to the C3 profile may have a weak prevention focus, which could affect their commitment to a long-term goal. To minimize this self-regulatory drawback, they may strengthen their prevention by reflecting on the consequence of giving up the important long-term goal. As a consequence, students may be able to ensure that all motivation orientations that are relevant to a goal pursuit are strong enough to both prompt positive selfregulatory tendencies and set constraints on one another. In turn, this could lead to effective self-regulation.

\section{Mapping motivational manifestations associated with different motivation}

profiles. The qualitative findings provide important empirical evidence with respect to motivational manifestations/characteristics associated with having different levels (i.e., strong vs. average vs. weak) of a motivation orientation. These findings also highlight how high (vs. moderate vs. low) levels on a given orientation are manifested in learning settings. I discuss these aspects below.

This research provides novel and important information with respect to motivational characteristics/tendencies associated with having average levels on assessment, locomotion, promotion, and prevention. Harring and Hodis (2016) noted that the extant literature has focused on key aspects related to having high (versus low) levels on these motivation 
orientations. Importantly, hypotheses regarding tendencies engendered by an average level of each of these orientations are not enunciated in current theorizing and substantive research. This study uncovered motivation profiles characterized by having average levels of one or more of the four motivation orientations investigated. The qualitative findings describing the motivation outlooks of the participants having these types of motivation profiles provide fresh insight into the typical motivational manifestations associated with having average levels on a given orientation. Particularly, these findings provide empirical evidence for the types of goals these individuals adopted, the types of strategic approaches they employed when engaging in learning, and their emotional responses to goal success/failure.

The qualitative findings in this study suggest that participants having average levels of a given motivation orientation exhibited tendencies/characteristics associated with both high and low levels on this orientation. For example, participants exhibiting the C2 profile had below average levels on assessment and locomotion. Two interviewees recruited from C2 tended to compare themselves to other people. All but one interviewee reported employing strategies that prioritize accuracy. In addition, two of them noted being indecisive because they evaluated different options before they made an important decision. All these tendencies reflected a strong assessment orientation (Higgins et al., 2003; Kruglanski, Pierro, \& Higgins, 2016; Kruglanski et al., 2000). In contrast, all four participants noted that they did not spend time comparing and critiquing the particular knowledge acquired or presented in their courses, which is a tendency that is typically associated with a weak assessment orientation (Higgins et al., 2003; Kruglanski et al., 2000). In a similar vein, two respondents reported preference to follow a sequence of consecutive steps when working on learning-related tasks; this tendency is typically associated with strong locomotion (Kruglanski et al., 2000; Pierro et al., 2011). All four interviewees also reported a lack of keenness to work towards new goals when they achieved a previous goal. All but one of the respondents stated that they were easily distracted during task engagement. Both of these tendencies reflect a weak locomotion orientation (Higgins et al., 2003; Pierro et al., 2011). In sum, the interview participants recruited from $\mathrm{C} 2$ acted as high assessors with regard to some aspects of their approaches to learning and as low assessors with regard to others. The same was true for locomotion.

As it was the case for their counterparts in $\mathrm{C} 2$, interview participants exhibiting the $\mathrm{C} 5$ 
profile - which was characterized by below average levels on assessment, locomotion, and prevention - also displayed tendencies related to both high and low levels on these orientations. Specifically, all five interview participants recalled investing effort to compare and evaluate the knowledge they learned in some of their courses. Two interviewees were inclined to engage in constant self-evaluations and experienced negative emotions. These tendencies are associated with strong assessment (Higgins et al., 2003; Kruglanski et al., 2000). Notably, these participants also displayed some propensities associated with weak assessment. For instance, all interviewees observed that they sometimes memorized what they were taught without critical evaluation. Three of these participants recalled that they seldom expended time to think about the optimal goals that they could adopt at university.

With regard to locomotion, two interview participants tended to work on their learningrelated tasks in a step-by-step manner; three interviewees noted that they switched goals when they experienced little progress in the goal pursuits. Both of these tendencies characterize a strong locomotion orientation (Higgins et al., 2003; Kruglanski et al., 2000). However, three of these interviewees found it difficult to maintain attention when they worked on a learning task, which is consistent with weak locomotion (Higgins et al., 2003). Finally, they showed characteristics associated with both strong and weak prevention orientations. Specifically, all five interviewees noted adopting prevention goals and employing some vigilant strategies to prevent failure in their goal pursuits. Three interview participants reported being motivated to live up to their parents' expectations regarding their academic achievement and meet the course requirements. All these characteristics pertain to strong prevention. In contrast, three interviewees reported being careless during task engagement, which is associated with weak prevention (Higgins, 1997, 2012).

Taking all of these aspects together, it could be concluded that having average levels of a given motivation orientation is likely to lead to tendencies/characteristics associated with both high and low levels on this orientation. The qualitative findings in this research also suggest that different levels on a given motivation orientation may be highlighted more strongly by the range of strategies the students employed than by the types of goals they adopted. The qualitative findings provide some illustrative examples in this sense. The interviewees, whose motivation profiles involved high, moderate or weak promotion, 
respectively, reported adopting promotion-related goals in learning at university. Specifically, all these students perceived learning at university as opportunities to attain their aspirations and achieve academic or career advancement. Importantly, the interviewees selected from $\mathrm{C} 1$ and C3, both of which were characterized by having high levels of promotion, generally reported using a wider range of strategies that are associated with strong promotion than their counterparts whose motivation profiles involved below average or low levels of promotion (i.e., C2, C4, and C5). Specifically, all but one of the students selected from C1 reported asking for help from both their teachers and peers and having an optimistic outlook when they faced difficulties in learning at university. All except one interviewees from C3 talked about using strategies that prioritized speed and their optimism in learning. One interview participant also discussed that she read beyond the course requirement with enthusiasm and sought help in the face of difficulties. These examples are typical of employing eager strategies in learning settings (Molden \& Rosenzweig, 2016).

In contrast, the interviewees recruited from $\mathrm{C} 2, \mathrm{C} 4$, and $\mathrm{C} 5$ (except for one) did not report using any eager strategies to achieve their promotion-related goals. Instead, they highlighted employing more vigilant strategies in their goal pursuits (e.g., following the course requirement, working on an assignment early, and checking their work carefully), which fit a strong prevention focus. These results suggest that although these students appeared to have promotion-related motivation, the motivational manifestations pertaining to promotion were somewhat less prevalent than the motivational manifestations corresponding to prevention. In light of these findings, it follows that to differentiate the consequences associated with having high vs. moderate vs. low levels on a motivation orientation, it is more important to examine the repertoire of strategies individuals employ than the types of goals they adopt. This conclusion provides novel and important information regarding distinct consequences associated with different strengths of a given motivation orientation.

Motivation profiles and key motivation factors/outcomes. This research compared similarities and differences among the five motivation profiles identified with regard to key motivation factors/outcomes, namely, grit, theories of intelligence, critical thinking, effort regulation, and evaluations/perceptions of academic success. The findings shed fresh light 
into the extent to which different patterns of regulatory focus and mode orientations (i.e., different motivation profiles) are associated with distinct levels of these factors/outcomes. Moreover, in mapping the structures of the motivation profiles to the theorizing and substantive results from regulatory focus and regulatory mode research, this study contributes to the existing motivation literature by unearthing novel and important information regarding the underpinnings of individual differences with regard to these outcomes.

Grit. Grit is conceptualized as a motivation construct comprising two facets (i.e., consistency of interest and perseverance of effort; Duckworth et al., 2007; Duckworth \& Quinn, 2009). Recent studies have provided empirical support for this conceptualization (Muenks et al., 2017; Steinmayr et al., 2018). As a result, in this research, the five motivation profiles were compared with regards to each of the two facets of grit separately.

The quantitative findings from this research showed that the $\mathrm{C} 1$ profile was associated with the highest mean levels of consistency of interest and perseverance of effort; the differences between $\mathrm{C} 1$ and the other profiles were statistically significant and moderate to large in magnitude. This result implies that the $\mathrm{C} 1$ profile was the most conducive to strong grit of all the profiles. This profile was characterized by strong/very strong levels on all four motivation orientations. This result is in line with Higgins' (2012) theorizing that motivation effects could be adaptive when the four orientations are all strong and operate effectively. Locomotion was found to be positively related to both perseverance of effort and consistency of interests (Mueller et al., 2017; Pierro et al., 2011). Thus, strong locomotion was likely to motivate the students exhibiting the $\mathrm{C} 1$ profile to maintain a course of action and persist consciensciously to overcome obstacles in goal pursuit. As a result, strong locomotion is likely to support perseverance of effort (Higgins, 2012; Mueller et al., 2017; Pierro et al., 2011). In addition, consistent with their strong locomotion, participants classified in $\mathrm{C} 1$ were likely to resist distractions and move toward their goal with focused attention. In turn, these tendencies contribute to exhibiting high consistency of interest (Higgins et al., 2003; Mueller et al., 2017; Pierro et al., 2011).

These positive tendencies associated with strong locomotion could constrain the negative characteristics associated with strong assessment (i.e., getting lost in excessive 
comparisons and evaluations). At the same time, strong promotion may drive these individuals to strive eagerly to maximize gains in their pursuit of promotion goals; in addition, strong prevention would motivate them to pursue their prevention goals vigilantly (Higgins, 2012). Together, these tendencies enabled these individuals to devote sustained effort in pursuits of important goals. Thus, high levels of promotion and prevention are likely to support strong perseverance of effort. More importantly, strong prevention sustained their commitment to their original important goal even in the face of obstacles and failures. This tendency could mitigate the proclivity to switch goals when they encountered difficulties, which is related to strong promotion (Molden, 2012; Molden \& Miele, 2008). Taken together, the motivational trade-offs among the four strong motivation orientations made these individuals likely to be gritty in goal pursuits.

As noted before, different levels of the two facets of grit were related to distinct patterns of associations of promotion, prevention, locomotion, and assessment (i.e., to different motivation profiles) (see details in the previous sections on inter-profile differences). In particular, when prevention was comparable, regardless of whether it was of strong or medium magnitude, higher levels of assessment, locomotion, and promotion were associated with stronger perseverance of effort and consistency of interest than lower levels of these three orientations.

In this research, large differences in the strength of prevention were associated with significant differences in levels of grit when assessment, locomotion, and promotion were all strong. Notably, in the motivation profile (i.e., C3) where the prevention focus was weak, prevention could neither play an important self-regulatory role nor constrain the negative tendencies associated with strong promotion and strong locomotion (Cornwell et al., 2015; Higgins, 2012). Consequently, when encountering difficulties, these students were unlikely to be very gritty in goal pursuits because their strong promotion and strong locomotion made them less committed to maintaining original goals and pursue other goals that could offer opportunities for advancement and progress.

In contrast, for the motivation profiles where assessment, locomotion, and promotion were comparable and of moderate magnitudes, variability in the strength of prevention was not associated with significant differences with regard to grit. In light of these findings and 
consistent with Higgins' (2012) theorizing, prevention alone could not influence the extent to which individuals are perseverant when encountering difficulties and committed to a longterm goal; rather, its role and function in shaping grit depended on the strength of the other orientations.

In sum, different patterns of interrelations among these motivation orientations, which were reflected by distinct motivation profiles, were associated with different levels of grit. Notably, when the four motivation orientations were simultaneously strong, they could interact effectively to support strong grit. These findings enhance understanding of interindividual differences in grit.

Theories of intelligence. This outcome measured students' beliefs regarding the nature of intelligence. Two different constructs capture this criterion, namely, incremental theory of intelligence and entity theory of intelligence. The quantitative findings indicated that with regard to both incremental and entity theories of intelligence, $\mathrm{C} 4$ had the highest mean levels among all profiles; however, the differences among the five profiles were generally small and, most often, not statistically significant. These findings suggest that for the sample in this research, differences in the motivation structures often did not translate into significant differences with regard to the theories of intelligence.

Critical thinking. This research used self-reported critical thinking and Cognitive Reflection Test (CRT) to evaluate similarities and differences among the motivation profiles. I discuss these two aspects in the following.

The quantitative findings revealed that $\mathrm{C} 3$ and $\mathrm{C} 1$ were associated with the highest mean levels of self-reported critical thinking among the five profiles. This suggests that $\mathrm{C} 3$ and $\mathrm{C} 1$ were the profiles most supportive of critical thinking in this research. Considering that these two motivation profiles were both characterized by having strong assessment, locomotion, and promotion, these findings indicate that high levels on these three orientations were associated with strong critical thinking. This conclusion is consistent with the results reported by Manalo et al. (2013). These authors examined the relationships among regulatory mode orientations (i.e., locomotion and assessment) and critical thinking. They reported that both assessment and locomotion were robust positive predictors of strong critical thinking (Manalo et al., 2013). 
For participants exhibiting the $\mathrm{C} 3$ and $\mathrm{C} 1$ profiles, strong locomotion was likely to motivate them to have a learning orientation (Higgins et al., 2003). Consistent with this orientation, to acquire knowledge and improve skills, they were likely to engage in deep processing, which characterizes critical thinking (e.g., critically analyzing new ideas and developing relationships and distinguishing between new ideas and previous concepts; Miele $\&$ Wigfield, 2014). At the same time, having strong assessment, they were likely to expend time and effort comparing and evaluating critically the knowledge they learned in their courses (Kruglanski et al., 2000; Manalo et al., 2013). Finally, having a strong promotion focus, these individuals were likely to connect topics of interests with information presented in their courses, and, therefore, to synthesize previous and new knowledge acquired (Crowe \& Higgins, 1997; Lockwood et al., 2002, 2004; Molden \& Miele, 2008). Taken together, these tendencies support strong critical thinking.

Similar to the case of grit, significant differences in self-reported critical thinking among the motivation profiles were also related with differences in motivation structures (see details in the sections on inter-profile differences). Specifically, for the motivation profiles in which assessment, locomotion and promotion were virtually equal (at high/average levels), variability in the magnitude of prevention was linked to significant differences in selfreported critical thinking. It is noteworthy that for these profiles, stronger prevention was associated with lower levels of self-reported critical thinking. The Critical Thinking Scale of the Motivated Strategies for Learning Questionnaire (used in this research to measure critical thinking) mainly focuses on how respondents use strategies of "applying previous knowledge to new situations in order to solve problems, reach decisions, or make critical evaluations with respect to standards of excellence" (Pintrich et al., 1993, p. 22). The findings suggest that employment of these types of critical thinking strategies (e.g., "Whenever I read or hear an assertion or conclusion in the class, I think about possible alternatives") was likely to negatively relate to prevention. A possible explanation is that during problem solving or decision making, prevention-oriented individuals are likely to narrow down the number of information they take into account, because considering a smaller amount of information helps them increase the likelihood of excluding incorrect information and minimize the chances of making errors (Molden \& Rosenzweig, 2016). Hence, they are less likely to use 
critical thinking strategies measured by the scale (e.g., thinking about alternatives when they learn an assertion in class).

Interestingly, these findings were not in line with the hypothesis enunciated by Miele and Wigfield (2014). These authors proposed that prevention is likely to have a positive association with critical thinking. This is the case because individuals having strong prevention orientations prefer slow, cautious, and analytical styles of information processing; therefore, they are inclined to use convergent thinking, which characterizes critical thinking. Moreover, deliberate and vigilant strategies that are involved in critical thinking could help prevention-oriented students protect against losses (Miele \& Wigfiled, 2014).

There were no significant differences among the five profiles with regard to CRT. As noted previously, the CRT may have been too easy for the participants in this research, perhaps leading to a ceiling effect. Nevertheless, the findings showed that the mean levels of CRT were the largest for the motivation profiles that were characterized by having high levels of prevention (i.e., C2 and C1), although the differences between these classes and the others were not statistically significant. This finding was in the direction proposed by Miele and Wigfield (2014).

To sum up, the results in this research provide new information regarding how different configurations of regulatory focus and mode orientations (i.e., motivation profiles) relate to students' reported use of critical thinking in learning-related tasks. Specifically, a combination of stronger (vs. weaker) assessment, locomotion, and promotion supported stronger self-reported critical thinking. When assessment, locomotion and promotion were comparable, regardless of whether they were at high or average levels, stronger prevention was associated with lower self-reported critical thinking.

Effort regulation. This criterion measured students' management of effort when engaged in challenging or uninteresting learning tasks. As it was the case for self-reported critical thinking, C3 and C1 had the highest mean levels of effort regulation among the five profiles. These two profiles are both characterized by having strong assessment, locomotion, and promotion. These results imply that high levels on these three orientations were linked to high levels of effort regulation. Particularly, strong locomotion was likely to motivate these individuals to have a strong task orientation (i.e., attending to a task and persisting until 
completion; Higgins et al., 2003; Kruglanski et al., 2000). Therefore, they tended to devote more effort and resist distractions when engaging in difficult or uninteresting learning related tasks (Pierro et al., 2011). Moreover, having a strong promotion focus, they were also more likely to study eagerly to maximize gains and advancement in these learning tasks (Higgins, 2012; Molden, 2012). All of these tendencies are conducive to strong effort regulation.

Importantly, for the motivation profiles where prevention was identical, regardless of whether it was at high or average levels, differences in the strengths of assessment, locomotion, and promotion were associated with significant differences in effort regulation. Therefore, it is likely that having concomitantly strong assessment, locomotion, and promotion orientations support effort regulation. This conclusion is similar to the corresponding one pertaining to self-reported critical thinking.

In this research, for the motivation profiles where assessment, locomotion, and promotion were almost identical and all strong (i.e., $\mathrm{C} 1$ and $\mathrm{C} 3$ ), large differences in the magnitude of prevention were not associated with significant differences in effort regulation. Variability in the magnitude of prevention was likely to translate into significant differences in effort regulation only when the remaining orientations were not simultaneously strong (i.e., $\mathrm{C} 2$ and $\mathrm{C} 5)$. Intriguingly, for the $\mathrm{C} 2$ and C5 profiles where the assessment, locomotion, and promotion were almost comparable and at average levels, stronger prevention (in the $\mathrm{C} 2$ profile) was associated with a lower level of effort regulation. A possible explanation of this finding is that in the $\mathrm{C} 2$ profile prevention dominated in strength over the other orientations. "When prevention motivation is extremely strong, it is associated with maladaptive responses that actually make it difficult for a person to achieve even their prevention goals" (Cornwell et al., 2015, p. 557). In addition, in C2 the other three orientations were too weak to engender positive self-regulatory tendencies (Cornwell et al., 2015; Higgins, 2012). Therefore, the students having the $\mathrm{C} 2$ profile were likely to have maladaptive responses (e.g., feeling overly anxious) when engaging in challenging tasks, and, thus, give up their effort. This is a plausible reason why these individuals displayed weak effort regulation.

To sum up, these findings highlight that differences in motivation structures (i.e., in motivation profiles) are related to differences in effort regulation. Specifically, a combination 
of higher levels on assessment, locomotion and promotion orientations was linked to higher levels of effort regulation. When none of these three orientations was strong, higher levels of prevention translated in lower levels of effort regulation. These findings provide support for theorizing that highlights the potential detrimental effects of having a dominant motivation orientation (Cornwell et al., 2015; Higgins, 2012; Scholer \& Higgins, 2012).

Perceptions/evaluations of academic success. This criterion captured students' selfreported evaluations of their academic achievement during the previous years at university. The highest average scores of perceptions/evaluations of academic success were associated with the motivation profiles characterized by having very high levels of prevention (i.e., C2 and $\mathrm{C} 1$ ); differences between these classes and the remaining ones were mostly significant and characterized by medium to large effect sizes. These findings suggest that the $\mathrm{C} 2$ and $\mathrm{C} 1$ profiles were the most supportive of having high perceptions/evaluations of academic achievement among the five profiles.

This conclusion is in the line with some empirical research on the relationships between regulatory focus and individuals' performance on analytical tasks (i.e., proofreading and reading comprehension; Förster et al., 2003; Miele, Molden, \& Gardner, 2009; Miele \& Wigfiled, 2014) and on standardized tests and college course exams (Rosenzweig \& Miele, 2016). Particularly, Rosenzweig and Miele (2016) reported that when prevention-focused college students had adequate time to complete the tests, they scored higher on the academic tests than did their counterparts who had strong promotion orientations. These authors also found that in the math part of a test, students having strong prevention orientations revisited more frequently the questions they had answered to check accuracy. Strong prevention motivated individuals to ensure against any potential losses. Consequently, they were more likely to employ vigilant strategies (e.g., being cautious and accurate; Higgins, 2012). In turn, this increased vigilance could contribute to better academic achievement, which then could be reflected in more elevated self-evaluations of academic success.

Importantly, when prevention was comparable (at high or average levels) in two profiles (i.e., C1 vs. C2 and C4 vs. C5), variations in the strengths of promotion, assessment, and locomotion were not associated with significant differences with regard to perceptions/evaluations of academic success. Moreover, high prevention was a stronger 
contributor to inter-profile differences in this outcome when the remaining orientations were not simultaneously strong than when they were all at high levels. Specifically, the C2 and C5 profiles had similar and average levels of promotion, assessment, and locomotion, but differed markedly in prevention. The quantitative results showed that $\mathrm{C} 2$ (characterized by having very high levels of prevention) was associated with much higher levels of perceptions/evaluations of academic success than C5 (encompassing below average levels of prevention). In contrast, C1 and C3 had comparable and high levels of assessment, locomotion, and promotion; prevention was very strong in $\mathrm{C} 1$, but was very weak in $\mathrm{C} 3$. The quantitative findings revealed that there was no statistically significant difference between these two profiles in perceptions/evaluations of academic success. Again, these findings are consistent with Higgins' (2012) theoretical proposition that the roles and effects of each motivation orientation depend on the strengths of the other three orientations; that is, promotion, prevention, assessment, and locomotion interact to shape motivation effects (Higgins, 2012; Hodis, 2018a; Hodis et al., 2017).

To conclude, the findings add to the knowledge about how the interrelationships among the motivation orientations influenced students' perceptions/evaluations of academic success. Notably, adopting stronger prevention is likely to be associated with stronger perceptions/evaluations of academic achievement.

The findings discussed in this section not only advance the theoretical and substantive knowledge on motivation and motivation effects, but also suggest some important implications for education practice. First, as discussed above, patterns of the associations among the motivation orientations (i.e., motivation profiles) could account for interindividual differences in key learning outcomes. Information provided by students' motivation profiles can help teachers/educators to more precisely predict individual differences in these outcomes. Second, knowledge of student's motivation profiles will be helpful to design intervention programmes that target and improve the key learning outcomes. For instance, inducing students to adopt a prevention orientation (e.g., by means of priming or task framing) while taking an untimed test can help them to be more vigilant against mistakes and possibly have a better test performance (Molden \& Rosenzweig, 2016; Wigfield et al., 2015). As it is relatively easy to temporarily induce individuals to adopt a 
motivation orientation (Higgins, 1997, 2012; Molden \& Rosenzweig, 2016), manipulations of students' motivation orientations could be "effective, viable ways to enhance achievement" (Wigfield et al., 2015, p. 15).

\section{Chapter Summary}

In this chapter, I integrated the quantitative and qualitative findings to provide an indepth description of the characteristics of each of the five motivation profiles identified in this study. Following, I evaluated the commonalities and differences among the profiles that had similar elements (i.e., having comparable levels on one or more of the motivation orientations). I also highlighted that the motivation profiles identified in the quantitative phase were collective profiles for the participants classified in the same class. Therefore, the interview participants shared some (but not all) of the characteristics associated with the profile in which they were classified. In addition, the qualitative findings showed that there were individual differences regarding tendencies associated with the motivation orientations among the interviewees who had the same motivation profile. In the final section of the chapter, I discussed the contributions the research makes to the existing literature and highlighted the important theoretical and practical implications of the findings. In the next chapter, I will conclude the thesis by summarizing the main findings and contributions of the research, discussing limitations of the study, and making recommendations for future research. 


\section{Chapter Seven Conclusion}

In this chapter, I provide first an overall summary of the research. Then, I review the limitations of the study and discuss potential avenues for future research. Finally, I highlight the main contributions this research has made to the motivation literature and educational practice.

\section{Summary of the Study}

In this section, the objectives, methodology, and key findings of the study are summarized briefly. This research sought to explore the number and nature of the motivation profiles, which reflected the interrelationships among the regulatory focus and mode orientations (i.e., promotion, prevention, assessment, and locomotion), for a sample of Chinese undergraduate students. An explanatory mixed methods approach was adopted. This research consisted of two distinct phases, with a quantitative study preceding a qualitative study.

Quantitative data were collected using paper questionnaires at a university in mainland China. Five distinct motivation profiles were identified. The characteristics of each of the profiles are summarized in Table 41. 
Table 41

A Summary of the Characteristics of the Five Motivation Profiles

\begin{tabular}{|c|c|c|c|c|c|}
\hline Profile & Assessment & Locomotion & Promotion & Prevention & Features of profiles \\
\hline $\mathrm{C} 1$ & High & High & High & Very high & $\begin{array}{l}\text { High/very high levels on all four orientations. Assessment, } \\
\text { locomotion, and promotion were comparable in strength, whereas } \\
\text { prevention was the strongest in this class; locomotion and promotion } \\
\text { were the strongest among all five profiles. }\end{array}$ \\
\hline $\mathrm{C} 2$ & $\begin{array}{l}\text { Below } \\
\text { average }\end{array}$ & $\begin{array}{l}\text { Below } \\
\text { average }\end{array}$ & $\begin{array}{l}\text { Below } \\
\text { average }\end{array}$ & Very high & $\begin{array}{l}\text { Below average and comparable levels of assessment, locomotion, } \\
\text { and promotion and very high levels of prevention. Prevention was } \\
\text { the strongest orientation in this class and had the highest level among } \\
\text { all five profiles. }\end{array}$ \\
\hline $\mathrm{C} 3$ & Very high & High & High & Very low & $\begin{array}{l}\text { High/very high assessment, locomotion, and promotion, but very low } \\
\text { prevention. Assessment, locomotion, and promotion were } \\
\text { comparable in strength in this profile. Prevention was the weakest } \\
\text { among all five profiles. }\end{array}$ \\
\hline $\mathrm{C} 4$ & Low & Very low & Very low & $\begin{array}{l}\text { Below } \\
\text { average }\end{array}$ & $\begin{array}{l}\text { Low/very low levels of assessment, locomotion, and promotion and } \\
\text { below average levels of prevention. Prevention was the strongest of } \\
\text { all orientations in this profile; assessment was slightly stronger than } \\
\text { locomotion and promotion which were of similar magnitude; } \\
\text { assessment, locomotion and promotion in C4 were the weakest } \\
\text { among all profiles. }\end{array}$ \\
\hline $\mathrm{C} 5$ & $\begin{array}{l}\text { Below } \\
\text { average }\end{array}$ & $\begin{array}{l}\text { Below } \\
\text { average }\end{array}$ & $\begin{array}{l}\text { Below } \\
\text { average }\end{array}$ & $\begin{array}{l}\text { Below } \\
\text { average }\end{array}$ & $\begin{array}{l}\text { Below average levels on all four orientations. Prevention was slightly } \\
\text { stronger than the other orientations; assessment, locomotion, and } \\
\text { promotion were virtually identical in strength in this profile. }\end{array}$ \\
\hline
\end{tabular}


Further, this research mapped the motivational significance of the five motivation profiles by comparing similarities/differences among the profiles with respect to key motivation factors that play critical roles in learning settings (i.e., grit, theories of intelligence, critical thinking, effort regulation, and perceptions/evaluations of academic success). The quantitative findings revealed that there were important differences among the five profiles with regard to most of these factors; the corresponding differences were characterized by moderate to large/very large effect sizes. Nevertheless, the differences among the five profiles with regard to theories of intelligence were generally small and, most often, not statistically significant. Moreover, there were no statistically significant differences among the profiles with regard to the Cognitive Reflection Test (CRT).

Several motivation profiles were found to support adaptive outcomes in learning settings. Generally, the $\mathrm{C} 1$ profile, which was characterized by high levels on the four orientations, was associated with relatively high (mean) levels on most criteria (with the exception of theories of intelligence). In addition, the $\mathrm{C} 2$ profile, which included very strong prevention and average levels on the other orientations, was associated with the strongest perceptions/evaluations of academic success. Moreover, the C3 profiles, which encompassed relatively strong promotion, locomotion, and assessment and very weak prevention, was most conducive to strong self-reported critical thinking and effort regulation. In contrast, the $\mathrm{C} 4$ profile, which was characterized by the low levels on the four tendencies, was associated with the lowest (mean) levels on most criteria among the five profiles. The C5 profile, which encompassed average levels on all four orientations, was related to medium or low levels on all motivation factors examined.

The qualitative phase of the research used a multiple-case study approach. Qualitative data were collected through semi-structured interviews; participants were selected from each motivation profile identified in the quantitative phase. The qualitative data added to the quantitative findings in that interview participants discussed how they approached learning at university. These data provided clear evidence that the learning approaches reported by the interview participants were generally congruent with the characteristics of their motivation profiles uncovered in the quantitative phase. 


\section{Limitations of the Study}

This research has several limitations that need to be noted. First, the data collected for this study were cross-sectional in nature; therefore, no causal inferences can be made. Another related limitation derives from the sampling methods. For convenience, participants in this study were drawn from a single provincial university in mainland China. Students' motivations may differ due to economic and other contextual factors that vary across geographical areas within a country (Chen \& Wong, 2015). Moreover, more female students participated in the survey than male students. This is why the sample in this research may not be representative of the entire population of Chinese undergraduate students. However, four motivation profiles identified in this study were structurally similar to the ones uncovered in Harring and Hodis (2016) and Hodis et al. (2017), which collected data from secondary school students in New Zealand. For example, the C3 profile in this research and the $\mathrm{C} 4$ profile in Hodis et al. (2017) were both characterized by strong/very strong assessment, locomotion, and promotion, but very weak prevention. This similarity suggests that this study captured appropriately configurations of general motivation tendencies. Hence, the results may generalize to undergraduate students from other universities in China. In addition, as noted before, interview participants in the qualitative phase of this study were volunteers who were willing to share their learning experiences; the majority of them reported high academic achievements. Thus, qualitative findings may reflect experiences of students who are relatively motivated and academically successful rather than the experiences of students who are less motivated/less successful. Further, more female students volunteered for the interviews than male students. Again, the sample in the qualitative phase may not be representative of the population of Chinese undergraduate students.

Some other limitations relate to the method of data collection. In particular, the questionnaires used in this study were administered in English. It is possible that as foreign language learners, some survey participants might have misunderstood some questionnaire items and given inaccurate responses. This might reduce the validity of the inferences made from quantitative data (Kelle, 2006). Nevertheless, the survey participants have been learning English as part of their degree programs and appeared to have little difficulty in 
understanding the English questionnaire items. In addition, the questionnaires were piloted with students who spoke English as a foreign language.

Second, this study used self-reports: questionnaires and interviews; therefore, the influence of method bias could not be ruled out (Creswell, 2008). For instance, it cannot be ruled out that some participants provided responses based on what they thought they should do, which may be different from what they actually did in academic settings. Thus, the study could have been strengthened if it had complemented the self-reported information with data from other sources (e.g., teachers, peers, and school records of grades). This aspect notwithstanding, some of the constructs examined in this study (e.g., regulatory focus/mode and theories of intelligence) can only be measured with self-reports.

Third, the Cognitive Reflection Test (CRT) appeared to have been too easy for the survey participants, which caused a ceiling effect. This is the most likely reason that the research did not find significant differences among the motivation profiles with regard to students' actual performance of critical thinking.

Finally, the quantitative findings in this study captured a snapshot of participants' motivation profiles at the moment when they filled in the questionnaires. During data collection at the target university, participants completed the questionnaires at different times within a two months period. This aspect could have affected the strength of some of their motivation orientations. For example, some students participated in the survey when a national English test was approaching. The upcoming academic test might have primed stronger prevention tendencies in these participants.

\section{Recommendations for Future Research}

In this section, I suggest some potentially productive directions for future research that could advance the motivation literature and address some of the limitations noted above. First, findings from this study provided strong empirical support for investigating the interrelationships among regulatory focus and regulatory mode orientations. Specifically, this study used latent profile analysis (LPA) to identify five distinct motivation profiles; these profiles captured the complex interplays among these motivation tendencies for a sample of 
Chinese undergraduate students. "As yet, there has been little research on the concept of motivational profiles or patterns (but see Hodis, Hattie, \& Hodis, 2017 for an exception). Nonetheless, the profiles concept offers multiple interesting ideas for future studies" (Pierro et al., 2018, p. 289). In light of this assertion, future research could investigate patterns of regulatory focus and mode orientations (i.e., motivation profiles) in other samples of Chinese undergraduate students. This future work could strengthen the generalizability of the findings.

As discussed in the previous chapter, a set of structurally similar motivation profiles has been consistently identified in this study and previous research (e.g., Harring \& Hodis, 2016; Hodis et al., 2017; Liu \& Yao, 2018), which collected data from populations with different cultural and/or developmental backgrounds. Hence, it would also be interesting to examine in future studies whether these structurally similar motivation profiles are replicated in other cultures/countries (e.g., the U.S. and Japan) and over a wider range of developmental ages (e.g., preadolescents and older adults).

Second, the English versions of Regulatory Focus Questionnaire (RFQ; Higgins et al., 2001) and Regulatory Mode Questionnaire (RMQ; Kruglanski et al., 2000) were adopted in this study. Future research could examine whether the translated versions of RFQ and RMQ are valid and reliable measures of the regulatory focus and mode orientations in Asian countries, where English is a second/foreign language (e.g., China). It would also be productive for future research to use these translated instruments to investigate whether Chinese undergraduate students exhibit motivation profiles similar to those uncovered in this research.

Third, as discussed previously, qualitative findings from this research showed that interview participants who had a combination of strong promotion and prevention reported being motivated by both success/positive feedback and failure/negative feedback. These findings are not in line with previous research (i.e., Shu \& Lam, 2011). Shu and Lam (2011) did not find that participants who exhibited both strong promotion and prevention were motivated by success and failure-related feedback. These researchers discussed that a likely reason for this case was that the self-regulatory influences of one regulatory focus dimension (e.g., promotion) could mitigate the effects of the other (e.g., prevention). For example, the motivation effects associated with the elation-related feelings experienced by individuals who 
have strong promotion and prevention levels and succeed at goal pursuits might be constrained by the accompanying calmness (which is connected to their high levels of prevention). Similarly, the anxiety and worry experienced by these individuals after failure might also be dampened by the sadness experienced at the same time (which is associated with their high levels of promotion) (Shu \& Lam, 2011). Relevant to this discussion, Higgins (2012) theorized that strong motivation orientations can both support and constrain one another in ways that lead to adaptive outcomes. Future research needs to examine how individuals who have high levels of both promotion and prevention respond to success/failure and positive/negative feedback. In so doing, these future studies could uncover new information about important motivational trade-offs among these motivation tendencies. Moreover, as noted before, this study used participants' self-reports. The interview participants might have reported what they thought they should do when facing success/failure and positive/negative feedback rather than what they actually did. To address this limitation, future research may use experimental designs or complement participants' self-reports with information from other sources (e.g., teachers and peers) to examine participants' responses to success/failure and positive/negative feedback.

Fourth, findings from this research showed that differences among the motivation profiles identified with respect to incremental and entity theories of intelligence were generally small and, most often, not statistically significant. It would be interesting for future studies to investigate whether these findings generalize to other samples from Chinese undergraduate students and other populations of students. No previous research has provided empirical evidence on the relationships between regulatory focus-mode motivation profiles and theories of intelligence. As such, future studies may examine the relationships between regulatory focus/mode orientations and theories of intelligence. This future work could shed light on motivation processes underlying individual differences with regard to theories of intelligence.

Fifth, the findings regarding similarities/differences among profiles with respect to self-reported critical thinking are not in the direction hypothesized by previous research (e.g., Miele \& Wigfield, 2014). Specifically, Miele and Wigfield (2014) proposed that prevention is likely to relate positively to critical thinking. In contrast, the findings in this study suggested 
that when assessment, locomotion, and promotion are virtually equal, stronger prevention is likely to be associated with lower levels of self-reported critical thinking. In this light, future studies are needed to use other validated scales of critical thinking to examine the relationships between the motivation profiles and critical thinking. In addition, as Cognitive Reflection Test appeared to have been too easy for this sample, researchers need to use more appropriate tests to measure students' actual performance of critical thinking in academic settings (e.g., in authentic classroom tasks).

Sixth, the construct of perceptions/evaluations of academic success examined in this study pertained to students' overall academic achievement during university years. Further work is needed to examine which patterns of motivation orientations support optimal academic performance in particular learning disciplines (e.g., English) or change during the cycle of the university year. To minimize the potential method bias, researchers could use objective data, (e.g., the school records of grades) in addition to self-reported information.

Finally, as motivation profiles capture the significant motivational states across individuals and cultures (Higgins, 2012; Hodis, 2018a), information on motivation profiles could help advance the knowledge on how the universal motivation orientations of promotion, prevention, locomotion, and assessment interact to shape individual differences in motivation effects. For example, in learning settings, investigating the between-profile differences with regard to outcomes of interest could shed light on the extent to which different patterns of interrelations among promotion, prevention, locomotion, and assessment relate to individual differences in these outcomes. Recent research has revealed significant relationships among regulatory focus/mode orientations and other important motivation constructs (e.g., expectancy of success, utility value, autonomous and controlled reasons for pursuing a goal, goal self-concordance, intrinsic and extrinsic motivations, and satisfaction of basic psychological needs; Hodis, 2018a, 2018b; Hodis \& Hodis, 2015, Koletzko, Herrmann, \& Brandstätter, 2015; Pierro et al., 2006; Vaughn, 2017). Hence, it would be productive for future research to explore the extent to which distinct patterns of regulatory focus and mode orientations (i.e., motivation profiles) account for significant differences with regard to other criteria (e.g., expectancies of success, utility value, intrinsic and extrinsic motivation). In addition, future work could identify which motivation profiles support adaptive outcomes in 
academic settings (e.g., strong and realistic expectancies of success and elevated intrinsic or utility value).

\section{Contributions of the Study}

This research explored the number and nature of the motivation configurations (i.e., motivation profiles) that mapped the interrelationships among four key motivation tendencies (i.e., promotion, prevention, assessment, and locomotion) for a sample of Chinese undergraduate students. In doing so, it contributes to the motivation literature and educational practice. In this section, I summarize these important contributions along three main foci: conceptual, substantive, and pedagogical.

At the conceptual level, this research advances understanding of motivation processes and attendant effects. The five motivation profiles uncovered in this study provided salient information on the interplays among promotion, prevention, locomotion, and assessment, which measured the three types of strivings for effectiveness. Moreover, distinct patterns of associations of the four motivation orientations (i.e., motivation profiles) accounted for individual differences regarding important motivation factors and outcomes.

Findings from this research also highlighted the important motivational trade-offs that shape motivation and self-regulation. As discussed in the previous chapter, the findings support two key theoretical tenets proposed by Higgins (2012), namely, that (a) motivation and motivation effects are shaped by the interrelationships among value, control, and truth effectiveness strivings; and (b) the self-regulatory roles and effects of each type of striving for effectiveness depend on the strengths of the other types of strivings. In addition, this study adds to the existing knowledge of characteristics/tendencies associated with the four motivation orientations. Specifically, findings in this study provide novel information regarding the cognitive, behavioral, and affective manifestations of exhibiting an average level of a given motivation orientation.

This study also makes important substantive contributions to the motivation literature. In particular, findings from the research suggest that the complex interrelationships among Chinese students' strivings for value, truth, and control effectiveness can be mapped by a set 
of distinct motivation profiles defined by the regulatory focus and mode orientations (for similar conclusions, see Hodis, 2018a). In addition, the motivation profiles identified in this study were similar in shape with their counterparts uncovered in studies in which data were collected from different cultural contexts and/or developmental ages (i.e., Harring \& Hodis, 2016; Hodis et al., 2017; Liu \& Yao, 2018). For example, the C3 profile in this research and the C4 profile in Hodis et al. (2017) both encompassed high levels of assessment, locomotion, and promotion, but very low levels of prevention. Moreover, in both my study and Hodis et al.'s (2017) research, these two profiles had the strongest assessment and the weakest prevention among all profiles identified in each respective sample. The identification of structurally similar profiles in two populations that differ with regard to both culture and developmental age suggests that these profiles capture appropriately key configurations that reflect the associations among these four general motivation tendencies. This is why, it is likely that these pattern of the motivation orientations could be identified in other countries/cultures (e.g., the U.S., Japan). Notably, there is a dearth of empirical research that explores the interrelationships among regulatory focus and regulatory mode orientations in Chinese or Asian contexts, which are at the center of this research. Hence, this study also advances substantive research by providing important insights into how these key orientations interacted to shape other motivation factors/effects for Chinese undergraduate students.

Apart from conceptual and substantive contributions, this study has important implications for pedagogy. Information on students' motivation profiles could help teachers understand better their students' motivation and identify effective pedagogical strategies. First, teachers who try to help students strengthen their academic performance may design features of learning environments (e.g., feedback and task instructions) in ways that support the characteristics of the learners exhibiting a given motivation profile. For example, for students who have a predominant prevention focus (e.g., the C2 profile that was characterized by very strong prevention and average levels of the remaining orientations), teachers may offer critical feedback and frame tasks in a loss/non-loss framework. As a consequence, these students could experience regulatory fit, and, thus, enhance their valuation of a given task, task engagement, and performance (Higgins, 2012; Molden \& Rosenzweig, 2016). 
In addition, teachers can use strategies to help students adjust productively their motivation orientations (e.g., temporarily enhancing one or more motivation tendencies that are adaptive for their learning and performance). By doing so, students are likely to "maintain the level and type of motivation needed to optimally pursue some goal" (Miele \& Scholer, 2018, p. 3). Case in point, results of this study showed that students having the C3 profile had high promotion, locomotion, and assessment, but a very weak prevention, which could made them less committed to a long-term goal, especially when they face difficulties or failure. Therefore, teachers may strengthen these students' prevention by using strategies such as asking them to think about the consequences of abandoning the important long-term goal. In doing so, students could stick to their important goals (rather than giving up) when encountering difficulties. Consequently, teachers may help students ensure that all motivation orientations that are relevant to a goal pursuit are strong enough to support optimal outcomes in learning settings (e.g., being gritty, persisting in difficult/uninteresting tasks).

Lastly, the findings from this study also suggest practical implications to learners. As I noted previously, it is helpful for students to monitor and control the strength (e.g., strong/weak) and type (e.g., promotion and prevention, assessment, and locomotion orientations) of their motivation during task engagement (Miele \& Scholer, 2018). As not all motivation tendencies are effective in supporting the requirements of a learning-related task, students who regulate their motivation tendencies to best suit particular task demands are likely to achieve optimal academic performance (Miele \& Scholer, 2018). For example, when these students feel that they are not strongly motivated or (when they experience a feelings of non-fit; i.e., a feeling that their current motivation does not match the task demands), they could adopt strategies that enhance their motivation or change into a motivation orientation that optimally supports their goal pursuits (Miele \& Scholer, 2018). In doing so, students are likely to be more engaged in the given task and perform better (Higgins, 2012; Miele \& Scholer, 2018).

In conclusion, this study has extended the existing literature on motivation and motivation effects. The findings from the research have supported Higgins' (2012) theorizing that motivation and its effects are influenced by the interrelationships among people's strivings for value, truth, and control effectiveness (which are reflected by promotion and 
prevention, assessment, and locomotion orientations, respectively). Moreover, the findings of the study are in line with some important results of the previous empirical studies (i.e., Harring \& Hodis, 2016; Hodis et al., 2017; Liu \& Yao, 2018) and bridged important gaps regarding the interrelationships among key motivation tendencies in Chinese/Asian contexts. The findings from this study could also inform teachers in their teaching practices and help learners attain success in their learning-related goal pursuits. Taken together, this research has made important contributions to both the knowledge of motivation and educational practice. 


\section{References}

Akaike, H. (1973). Information theory and an extension of the maximum likelihood principle. In B. N. Petrov, \& F. Csaki (Eds.), Second international symposium on information theory (pp. 267-281). Budapest, Hungary: Akademiai Kiado.

Akhtar, S., \& Lee, J. S. Y. (2014). Assessing factor structure and convergent validity of the work regulatory focus scale. Psychological Reports, 115, 133-147. doi: 10.2466/08.01.PR0.115c13z5

Arbuckle, J. L. (1996). Full information in the presence of incomplete data. In G. A. Marcoulides \& R. E. Schumacker (Eds.), Advanced structural equation modeling: Issues and techniques (pp. 243-277). Mahwah, NJ: Lawrence Erlbaum Associates.

Asparouhov, T., \& Muthén, B. O. (2014). Auxiliary variables in mixture modeling: Three step approaches using Mplus. Structural Equation Modeling: A Multidisciplinary Journal, 21,329-341. doi:10.1080/10705511.2014.915181

Barrick, M. R., \& Mount, M. K. (1991). The big five personality dimensions and job performance: A metaanalysis. Personnel Psychology, 44, 1-26. doi: 10.1111/j.1744-6570.1991.tb00688.x

Bentler, P. M. (1990). Comparative fit indexes in structural models. Psychological Bulletin, 107, 238-246. doi:10.1037//0033-2909.107.2.238

Bentler, P.M. (1995). EQS: Structural equations program manual. Encino, CA: Multivariate Software.

Bidjerano, T., \& Dai, D. Y. (2007). The relationship between the big-five model of personality and self-regulated learning strategies. Learning and Individual Differences, 17, 69-81. doi: 10.1016/j.lindif.2007.02.001

Braun, V., \& Clarke, V. (2006). Using thematic analysis in psychology. Qualitative Research in Psychology, 3, 77-101. doi: 10.1191/1478088706qp063oa

Brown, T. A. (2006). Confirmatory factor analysis for applied research. New York, NY: Guilford.

Byrne, B. M. (2011). Structural equation modeling with Mplus. New York. NY: Routledge. 
Byrnes, J. P. \& Dunbar, K. N. (2014). The nature and development of critical-analytic thinking. Educational Psychology Review, 26, 477-493.

doi: $10.1007 / \mathrm{s} 10648-014-9284-0$

Carver, C. S., \& Scheier, M. F. (1981). Attention and self-regulation: A control theory approach to human behavior. New York, NY: Springer.

Chan, S. (1999). The Chinese learner: A question of style. Education \& Training, 41, 294304. doi: 10.1108/00400919910285345

Chen, W. W., \& Wong, Y. L. (2015). Chinese mindset: Theories of intelligence, goal orientation and academic achievement in Hong Kong students. Educational Psychology, 35, 714-725. doi: 10.1080/01443410.2014.893559

Chen, Y., Wen, Z., \& Ye, M. (2017). Exploring profiles of work regulatory focus: A personcentered approach. Personality and Individual Differences, 116, 16-21. doi: $10.1016 / j$. paid.2017.04.019

Cheung, A. C. K. (2013). Language, academic, socio-cultural and financial adjustments of mainland Chinese students studying in Hong Kong. International Journal of Educational Management, 27, 221-241. doi: 10.1108/09513541311306459

Clark, L. A., \& Watson, D. (1995). Constructing validity: Basic issues in objective scale development. Psychological Assessment, 7, 309-319. doi:10.1037/1040-3590.7.3.309

Cohen, L., Manion, L., \& Morrison, K. (2011). Research methods in education. New York, NY: Routledge.

Conley, A. M. (2012). Patterns of motivation beliefs: Combining achievement goal and expectancy-value perspectives. Journal of Educational Psychology, 104, 32-47. doi: $10.1037 / \mathrm{a} 0026042$

Cormier, G. (2018). The language variable in educational research: An exploration of researcher positionality, translation, and interpretation. International Journal of Research and Method in Education, 41, 328-341. doi: 10.1080/1743727X.2017.1307335

Cornwell, J. F., Franks, B., \& Higgins, E. T. (2015). Distress from motivational disintegration: When fundamental motives are too weak or too strong. In E. H. Simpson \& P. D. Balsam (Series Eds.), Current topics in behavioral neuroscience: Vol. 27. 
Behavioral neuroscience of motivation (pp. 547-568). doi: 10.1007/7854_2015_389

Cortazzi, M., Pilcher, N. \& Jin, L. (2011). Language choices and "blind shadows":

Investigating interviews with Chinese participants. Qualitative Research, 11, 505535. doi: $0.1177 / 1468794111413225$

Costa, P. T., \& McCrae, R. R. (1992). Normal personality assessment in clinical practice: The NEO personality inventory. Psychological Assessment, 4, 5-13.

doi: $10.1037 / 1040-3590.4 .1 .5$

Credé, M. A. (2018). What shall we do about grit? A critical review of what we know and what we don't know. Educational Researcher, 47, 606-611. doi: 10.3102/0013189X18801322

Credé, M. A., \& Phillips, L. A. (2011). A meta-analytic review of the Motivated Strategies for Learning Questionnaire. Learning and Individual Differences, 21, 337-346. doi: 10.1016/j.lindif.2011.03.002

Credé, M. A., Tynan, M. C., \& Harms, P. D. (2017). Much ado about grit: A meta-analytic synthesis of the grit literature. Journal of Personality and Social Psychology, 113, 492-511. doi: 10.1037/pspp0000102

Creswell, J. W. (2008). Educational research: Planning, conducting, and evaluating quantitative and qualitative research (3rd ed.). Upper Saddle River, NJ: Pearson Prentice Hall.

Creswell, J. W. (2014). Research design: Qualitative, quantitative, and mixed methods approaches (4th ed.). Thousand Oaks, CA: Sage.

Creswell, J. W., \& Plano Clark, V. L. (2011). Designing and conducting mixed method research (2nd ed.). Thousand Oaks, CA: Sage.

Crowe, E., \& Higgins, E. T. (1997). Regulatory focus and strategic inclinations: Promotion and prevention in decision-making. Organizational Behavior and Human Decision Processes, 69, 117-132. doi: 10.1006/obhd.1996.2675

Curran, P. J., West, S. G., \& Finch, J. F. (1996). The robustness of test statistics to nonnormality and specification error in confirmatory factor analysis. Psychological Methods, 1, 16-29. doi: 10.1037/1082989X.1.1.16

Duckworth, A. L., \& Gross, J. J. (2014). Self-control and grit: Related but separable 
determinants of success. Current Directions in Psychological Science, 23, 319-325. doi: $10.1177 / 0963721414541462$

Duckworth, A. L., Peterson, C., Matthews, M. D., \& Kelly, D. R. (2007). Grit:

Perseverance and passion for long-term goals. Journal of Personality and Social Psychology, 92, 1087-1101. doi: 10.1037/0022-3514.92.6.1087

Duckworth, A. L., \& Quinn, P. (2009). Development and validation of the Short Grit Scale (GRIT-S). Journal of Personality Assessment, 91, 166-174.

doi: 10.1080/00223890802634290

Dweck, C. S. (1999). Self-theories: Their role in motivation, personality, and development. Philadelphia, PA: Psychology Press.

Dweck, C. S., Chiu, C. \& Hong, Y. (1995). Implicit theories and their role in judgments and reactions: A world from two perspectives. Psychological Inquiry, 6, 267-285. doi: 10.1207/s15327965pli0604_1

Dweck, C. S., Higgins, E. T., \& Grant-Pillow, H. (2003). Self-systems give unique meaning to self-variables. In M. R. Leary \& J. P. Tangney (Eds.), Handbook of self and identity (pp. 239-252). New York, NY: Guilford.

Dweck, C. S. \& Leggett, E. L. (1988). A social-cognitive approach to motivation and personality. Psychological Review, 95, 256-273. doi: 10.1037/0033-295X.95.2.256

Eccles, J. S., Adler, T. F., Futterman, R., Goff, S. B., Kaczala, C. M., Meece, J. I., \& Midgley, C. (1983). Expectancies, values, and academic behaviors. In J. T. Spence (Ed.), Achievement and achievement motives: Psychological and sociological approaches (pp. 75-145). San Francisco, CA: Freeman.

Eccles, J. S., \& Wigfield, A. (2002). Motivational beliefs, values, and goals. Annual Review of Psychology, 53, 109-132. doi: 10.1146/annurev. psych.53.100901.135153

Eitam, B., Miele, D. B. \& Higgins, E. T. (2013). Motivated remembering: Remembering as accessibility and accessibility as motivational relevance. In D. Carlston (Ed.), The Oxford handbook of social cognition (pp. 464-474). doi: 10.1093/oxfordhb/9780199730018.013.0022

Elliot, A., \& Covington, M. (2001). Approach and avoidance motivation. Educational 
Psychology Review, 13, 73-92. doi: 10.1023/A:1009009018235

Elliott, E. S., \& Dweck, C. S. (1988). Goals: An approach to motivation and achievement. Journal of Personality and Social Psychology, 54, 5-12. doi: 10.1037/0022-3514.54.1.5

Eskreis-Winkler, L., Shulman, E. P., Beal, S. A., \& Duckworth, A. L. (2014). The grit effect: Predicting retention in the military, the workplace, school and marriage. Frontiers in Psychology, 5, 1-12. doi: 10.3389/fpsyg.2014.00036

Fan, X., \& Wang, L. (1998). Effects of potential confounding factors on fit indices and parameter estimates on true and misspecified SEM models. Educational and Psychological Measurement, 58, 701-735. doi:10.1177/0013164498058005001

Feng, A. (2011). Introduction: The apex of 'the third wave' - English language across Greater China. In A. Feng (Ed.), English language education cross Greater China. New York, NY: Multilingual Matters.

Förster, J., Higgins, E. T., \& Bianco, A. T. (2003). Speed/accuracy decisions in task performance: Built-in trade-off or separate strategic concerns? Organizational Behavior and Human Decision Processes, 90, 148-164.

doi: 10.1016/S0749-5978(02)00509-5

Franks, B., \& Higgins, E. T. (2012). Effectiveness in humans and other animals: A common basis for well-being and welfare. In J. M. Olson \& M. P. Zanna (Eds.), Advances in experimental social psychology (Vol. 46, pp. 285-346). New York, NY: Academic Press.

Frederick, S. (2005). Cognitive reflection and decision making. The Journal of Economic Perspectives, 19, 25-42. doi: 10.1257/089533005775196732

Friedman, R. S., \& Förster, J. (2001). The effects of promotion and prevention cues on creativity. Journal of Personality and Social Psychology, 81, 1001-1013. doi: 10.1037/0022-3514.81.6.1001

Geiser, C. (2013). Data analysis with Mplus. New York, NY: Guilford.

Gorges, J., \& Göke, T. (2015). How do I know what I can do? Anticipating expectancy of success regarding novel academic tasks. British Journal of Educational Psychology, 85, 75-90. doi: 10.1111/bjep.12064 
Grant, H., \& Dweck, C. S. (2003). Clarifying achievement goals and their impact. Journal of Personality and Social Psychology, 85, 541-553. doi: 10.1037/0022-3514.85.3.541

Greene, J. A., Costa, L.-J., Robertson, J., Pan, Y., \& Deekens, V. M. (2010). Exploring relations among college students' prior knowledge, implicit theories of intelligence, and selfregulated learning in a hypermedia environment. Computers \& Education, 55, $1027-$ 1043. doi: 10.1016/j.compedu.2010.04.013

Harring, J. R., \& Hodis, F. A. (2016). Mixture modeling: Applications in educational psychology. Educational Psychologist, 51, 354-367. doi: $10.1080 / 00461520.2016 .1207176$

Hennan, M. R., Dornbusch, S. M., Herron, M. C., \& Herting, J. R. (1997). The influence of family regulation, connection, and psychological autonomy on six measures of adolescent functioning. Journal of Adolescent Research, 12, 34-67. doi: $10.1177 / 0743554897121004$

Hesse-Biber, S. N. (2010). Mixed methods research: Merging theory with practice. New York, NY: Guilford.

Higgins, E. T. (1987). Self-discrepancy: A theory relating self and affect. Psychological Review, 94, 319-340. doi: 10.1037/0033-295X.94.3.319

Higgins, E. T. (1997). Beyond pleasure and pain. American Psychologist, 52, 1280-1300. doi: 10.1037/0003-066X.52.12.1280

Higgins, E. T. (2008). Culture and personality: Variability across universal motives as the missing link. Social and Personality Psychology Compass, 2, 608-634. doi: 10.1111/j. 1751-9004.2007.00075.x

Higgins, E. T. (2012). Beyond pleasure and pain: How motivation works. New York, NY: Oxford University Press.

Higgins, E. T. (2013). Truth motivation. In K. D. Markman, T. Proulx, \& M. J. Lindberg (Eds.), The psychology of meaning (pp. 91-114). doi: 10. 1037/14040-005

Higgins, E. T. (2014). Promotion and prevention: How “0” can create dual motivational forces. In J. W. Sherman, B. Gawronski, \& Y. Trope (Eds.), Dual-process theories of the social mind (pp. 423-435). New York, NY: Guilford.

Higgins, E. T., Cornwell, J. F. M., \& Franks, B. (2014). "Happiness" and "the good life" as 
motives working together effectively. In A. J. Elliot (Ed.), Advances in motivation science (Vol. 1, pp. 135-179). doi: 10. 1016/bs.adms. 2014. 08.004

Higgins, E. T., Friedman, R. S., Harlow, R. E., Idson, L. C., Ayduk, O. N., \& Taylor, A. (2001). Achievement orientations from subjective histories of success: Promotion pride versus prevention pride. European Journal of Social Psychology, 31, 3-23. doi: $10.1002 /$ ejsp. 27

Higgins, E. T., Kruglanski, A. W., \& Pierro, A. (2003). Regulatory mode: Locomotion and assessment as distinct orientations. In M. P. Zanna (Ed.), Advances in experimental social psychology (Vol. 35, pp. 293-344). New York, NY: Academic Press.

Higgins, E. T., Pierro, A., \& Kruglanski, A. W. (2008). Re-thinking culture and personality: How self-regulatory universals create cross-cultural differences. In R. Sorrentino \& A. Yamaguchi (Eds.), Handbook of motivation and cognition across cultures (pp. 161190). San Diego, CA: Elsevier.

Higgins, E. T., \& Scholer, A. A. (2015). Goal pursuit functions: Working together. In M. Mikulincer \& P. R. Shaver (Eds.), APA handbook of personality and social psychology: Vol. 1. Attitudes and social cognition (pp. 843-889). doi: 10. 1037/14341-027

Ho, E., Holmes, P., \& Cooper, J. (2004). Review and evaluation of international literature on managing cultural diversity in the classroom. Wellington, New Zealand: Ministry of Education.

Hodis, F. A. (2015). A multi-sample investigation of the assessment and locomotion scales in a population of secondary school students. Journal of Psychoeducational Assessment, 33, 130-140. doi: 10.1177/0734282914547871

Hodis, F. A. (2017). Investigating the structure of regulatory focus: A bifactor analysis. Personality and Individual Differences, 109, 192-200. doi: 10.1016/j.paid.2017.01.004

Hodis, F. A. (2018a). Examining individuals' strivings for value, control, and truth effectiveness: Implications for educational psychology research. Educational Psychological Review, 30, 1001-1030. doi: 10.1007/s10648-018-9439-5

Hodis, F. A. (2018b). Underpinnings of expectancies of success in mathematics: An analysis 
of general, school-related, and domain-specific motivation antecedents. Journal of Educational Psychology, 110, 407-430. doi: 10.1037/edu0000218

Hodis, F. A., Hattie, J. A.C., Hodis, G. M. (2017). Investigating student motivation at the confluence of multiple effectiveness strivings: A study of promotion, prevention, locomotion, assessment, and their interrelationships. Personality and Individual Differences, 109, 181-191. doi: 10.1016/j.paid.2017.01.009

Hodis, F. A., \& Hodis, G. M. (2013). Latent growth modeling for communication research: Opportunities and perspectives. Annals of the International Communication Association, 37, 183-218. doi:10.1080/23808985.2013.11679150

Hodis, F. A., \& Hodis, G. M. (2015). Expectancy, value, promotion, and prevention: An integrative account of regulatory fit vs. non-fit with student satisfaction in communicating with teachers. Annals of the International Communication Association, 39, 339-370. doi: 10.1080/23808985.2015.11679180

Hodis, F. A., \& Hodis, G. M. (2017). Assessing motivation of secondary school students: An analysis of promotion and prevention orientations as measured by the regulatory focus questionnaire. Journal of Psychoeducational Assessment, 35, 670-682. doi: $10.1177 / 0734282916658385$

Hodis, F. A., Meyer, L. H., McClure, J., Weir, K. F., \& Walkey, F. (2011). A longitudinal investigation of motivation and secondary school achievement using growth mixture modeling. Journal of Educational Psychology, 103, 312-323. doi: 10.1037/a0022547

Hong, R. Y., Tan, M. S., \& Chang, W. C. (2004). Locomotion and assessment: Self-regulation and subjective well-being. Personality and Individual Differences, 37, 325-332. doi: 10.1016/j.paid.2003.09.006

Hong, Y., Chiu, C., Dweck, C. S., Lin, D., M. S., \& Wan, W. (1999). Implicit theories, attributions, and coping: A meaning system approach. Journal of Personality and Social Psychology, 77, 588-599. doi: 10.1037/0022-3514.77.3.588

Ip, G. W. M., Chen, J., \& Chiu, C. Y. (2006). The relationship of promotion focus, need for cognitive closure, and categorical accessibility in American and Hong Kong Chinese university students. Journal of Creative Behavior, 40, 201-215. doi: 10.1002/j.2162-6057.2006.tb01273.x 
Janosz, M., Archambault, I., Morizot, J., \& Pagani, L. (2008). School engagement trajectories and their differential predictive relations to dropout. Journal of Social Issues, 64, 2140. doi: 10.1111/j.1540-4560.2008.00546.x

Jin, X., Wang, L., \& Dong, H. (2016). The relationship between self-construal and creativity—Regulatory focus as moderator. Personality and Individual Differences, 97, 282-288. doi: 10.1016/j.paid.2016.03.044

Johnson, B., \& Christensen, L. (2014). Educational research: Quantitative, qualitative, and mixed approaches (5th ed.). Thousand Oaks, CA: Sage.

Johnson, B., \& Christensen, L. (2017). Educational research: Quantitative, qualitative, and mixed approaches (6th ed.). Thousand Oaks, CA: Sage.

Jones, B. D., Wilkins, J. L. M., Long, M. H., \& Wang, F. (2012). Testing a motivational model of achievement: How students' mathematical beliefs and interests are related to their achievement. European Journal of Psychology of Education, 27, 1-20. doi: 10.1007/s10212-011-0062-9

Jöreskog, K.G. (1969). A general approach to confirmatory maximum likelihood factor analysis. Psychometrika, 34, 182-202.

Jung, T., \& Wickrama, K.A.S. (2008). An introduction to latent class growth analysis and growth mixture modeling. Social and Personality Psychology Compass, 2, 302-17. doi: 10.1111/j.1751-9004.2007.00054.x

Kelle, U. (2006). Combining qualitative and quantitative methods in research practice: Purposes and advantages. Qualitative Research in Psychology, 3, 293-311. doi: $10.1177 / 1478088706070839$

Kline, R. B. (2005). Principles and practice of structural equation modeling (2nd ed.). New York, NY: Guilford.

Koletzko, S. H., Herrmann, M., \& Brandstätter, V. (2015). Unconflicted goal striving: Goal ambivalence as mediator between goal self-concordance and well-being. Personality and Social Psychology Bulletin, 41, 140-156. doi: 10.1177/0146167214559711

Komarraju, M., \& Nadler, D. (2013). Self-efficacy and academic achievement: Why do implicit beliefs, goals, and effort regulation matter? Learning and Individual Differences, 25, 67-72. doi: 10.1016/j.lindif.2013.01.005 
Kooij, D. T. A. M., Bal, P. M., \& Kanfer, R. (2014). Future time perspective and promotion focus as determinants of intraindividual change in work motivation. Psychology and Aging, 29, 319-328. doi: 10.1037/a0036768

Kruglanski, A. W., Orehek, E. , Higgins, E. T., Pierro, A., \& Shalev, I. (2010). Modes of selfregualtion: Assessment and locomotion as independent determinants in goal-pursuit. In R. Hoyle (Ed.), Handbook of personality and self-regulation (pp. 375-402). Oxford, UK: Wiley-Blackwell. doi: 10.1002/9781444318111.ch17

Kruglanski, A. W., Pierro, A., \& Higgins, E. T. (2016). Experience of time by people on the go: A theory of the locomotion-temporality interface. Personality and Social Psychology Review, 20, 100-117. doi: 10.1177/1088868315581120

Kruglanski, A. W., Thompson, E. P., Higgins, E. T., Atash, M. N., Pierro, A., Shah, J. Y., \& Spiegel, S. (2000). To "do the right thing" or to "just do it": Locomotion and assessment as distinct self-regulatory imperatives. Journal of Personality and Social Psychology, 79, 793-815. doi: 10.1037//0022-3514.79.5.793

Kurman, J., \& Hui, C. M. (2012). Cultural regulatory fit and strategies for coping with unsuccessful outcomes. European Journal of Social Psychology, 42, 482-489. doi: $10.1002 /$ ejsp.1838

Kurman, J., Liem, G. A., Ivancovsky, T., Morio, H., \& Lee, J. (2015). Regulatory focus as an explanatory variable for cross-cultural differences in achievement-related behavior. Journal of Cross-Cultural Psychology, 46, 171-190. doi: $10.1177 / 0022022114558090$

Lam, T. W. H., \& Chiu, C. Y. (2002). The motivational function of regulatory focus in creativity. The Journal of Creative Behavior, 36, 138-150.

Lee, A. Y., Aaker, J. L., \& Gardner, W. L. (2000). The pleasures and pains of distinct selfconstruals: The role of interdependence in regulatory focus. Journal of Personality and Social Psychology, 78, 1122-1134. doi: 10.1037/0022-3514.78.6.1122

Li, C. H., \& Wu, J. J. (2011). The structural relationships between optimism and innovative behavior: Understanding potential antecedents and mediating effects. Creativity Research Journal, 23, 119-128. doi: 10.1080/10400419.2011.571184

Lisjak, M., Molden, D. C., \& Lee, A. Y. (2012). Primed interference: The cognitive and 
behavioral costs of an incongruity between chronic and primed motivational orientations. Journal of Personality and Social Psychology, 102, 889-909. doi: $10.1037 / \mathrm{a} 0027594$

Liu, H., \& Yao, M. (2018). Regulatory focus profiles among Chinese pre-adolescents and adolescents and their relationship to personality and psychological outcomes. Journal of Happiness Studies, 1-18. doi: 10.1007/s10902-018-0025-9

Lo, Y., Mendall, N. R., \& Rubin, D. B. (2001). Testing the number of components in a normal mixture. Biometrika, 88, 767-778. doi: 10.1093/biomet/88.3.767

Lochmiller, C. R., \& Lester, J. N. (2015). An introduction to educational research: Connecting methods to practice. Singapore: Sage.

Lockwood, P., Jordan, C. H., \& Kunda, Z. (2002). Motivation by positive or negative role models: Regulatory focus determines who will best inspire us. Journal of Personality and Social Psychology, 83, 854-864. doi: 10.1037/0022-3514.83.4.854

Lockwood, P., Marshall, T. C., \& Sadler, P. (2005). Promoting success or preventing failure: Cultural differences in motivation by positive and negative role models. Personality \& Social Psychology Bulletin, 31, 379-392. doi: 10.1177/0146167204271598

Lockwood, P., Sadler, P., Fyman, K., \& Tuck, S. (2004). To do or not to do: Using positive or negative role models to harness motivation. Social Cognition, 22, 422-450. doi: $10.1521 /$ soco.22.4.422.38297

Manalo, E., Kusumi, T., Koyasu, M., Michita, Y., \& Tanaka, Y. (2013). To what extent do culture-related factors influence university students' critical thinking use? Thinking Skills and Creativity, 10, 121-132. doi: 10.1016/j.tsc.2013.08.003

Mann, S. (2016). The research interview: Reflective practice and reflexivity in research processes. London, UK: Palgrave Macmillan.

Mann, T., de Ridder, D., \& Fujita, K. (2013). Self-regulation of health behavior: Social psychological approaches to goal setting and goal striving. Health Psychology, 32, 487-498. doi:10.1037/a0028533

Markus, H. R., \& Kitayama, S. (1991). Culture and the self: Implications for cognition, emotion, and motivation. Psychological Review, 98, 224-253. doi: 10.1037/0033-295X.98.2.224 
Mathur, P., Chun, H. H. \& Maheswaran, D. (2016). Consumer mindsets and self-enhancement: Signaling versus learning. Journal of Consumer Psychology, 26, 142-152. doi: 10.1016/j.jcps.2015.06.007

McLachlan, G. J., \& Peel, D. (2000). Finite mixture models. New York, NY: Wiley.

Merriam, S.B. (1998). Qualitative research and case study applications in education. Revised and expanded from "Case study research in education”. San Francisco, CA: JosseyBass.

Mertens, D. (2006). Research and evaluation in education and psychology: Integrating diversity with quantitative, qualitative and mixed methods (3rd ed.). Thousand Oaks, CA: Sage.

Meyer, L. H., McClure, J., Walkey, F., Weir, K. F., \& McKenzie, L. (2009). Secondary student motivation orientations and standards-based achievement outcomes. British Journal of Educational Psychology, 79, 273-293. doi: 10.1348/000709908X354591

Miele, D.B., Molden, D.C., \& Gardner, W.L. (2009). Motivated comprehension regulation: Vigilant versus eager metacognitive control. Memory and Cognition, 37, 779-795. doi: 10.3758/MC.37.6.779

Miele, D. B., \& Scholer, A. A. (2018). The role of metamotivational monitoring in motivation regulation. Educational Psychologist, 53, 1-21. doi:10.1080/00461520.2017.1371601

Miele, D. B., \& Wigfield, A. W. (2014). Quantitative and qualitative relations between motivation and critical-analytic thinking. Educational Psychology Review, 26, 519541. doi: 10.1007/s10648-014-9282-2

Molden, D. C. (2012). Motivated strategies for judgment: How preferences for particular judgment processes can affect judgment outcomes. Social and Personality Psychology Compass, 6, 156-169. doi: 10.1111/j.1751-9004.2011.00424.x

Molden, D. C., \& Higgins, E. T. (2012). Motivated thinking. In K. J. Holyoak \& R. G. Morrison (Eds.), The Oxford handbook of thinking and reasoning (pp. 390-409). New York, NY: Oxford University Press.

Molden, D. C., Lee, A. Y., \& Higgins, E. T. (2008). Motivation for promotion and prevention. In J. Y. Shah \& W. L. Gardner (Eds.), Handbook of motivation science (pp. 169-187). New York, NY: Guilford. 
Molden, D. C., \& Miele, D. B. (2008). The origins and influences of promotion-focused and prevention-focused achievement motivations. In M. Maehr, S. Karabenick, \& T. Urdan (Eds.), Advances in motivation and achievement: Vol. 15. Social psychological perspectives (pp. 81-118). Bingley, UK: Emerald.

Molden, D. C., \& Rosenzweig, E. Q. (2016). The origins and educational implications of promotion-focused and prevention-focused achievement motivations. In K. R. Wentzel \& D. B. Miele (Eds.), Handbook of motivation at school (2nd ed., pp. 477503). New York, NY: Routledge.

Morgan, D. L. (2007). Paradigms lost and pragmatism regained methodological implications of combining qualitative and quantitative methods. Journal of Mixed Methods Research, 1, 48-76. doi: 10.1177/2345678906292462

Mueller, B. A., Wolfe, M. T., \& Syed, I. (2017). Passion and grit: An exploration of the pathways leading to venture success. Journal of Business Venturing, 32, 260-279. doi:10.1016/j.jbusvent.2017.02.001

Muenks, K., Wigfield, A., Yang, J. S., \& O’Neal, C. R. (2017). How true is grit? Assessing its relations to high school and college students' personality characteristics, selfregulation, engagement, and achievement. Journal of Educational Psychology, 109, 599-620. doi: 10.1037/edu0000153

Muenks, K., Yang, J. S., \& Wigfield, A. (2018). Associations between grit, motivation, and achievement in high school students. Motivation Science, 4, 158-176. doi:10.1037/mot0000076

Musu-Gillette, L. E., Wigfield, A., Harring, J. R., \& Eccles, J. S. (2015). Trajectories of change in students' self-concepts of ability and values in math and college major choice. Educational Research and Evaluation, 21, 343-370.

doi: $10.1080 / 13803611.2015 .1057161$

Muthén, L.K., \& Muthén, B.O. (2017). Mplus user's guide (8th ed.). Los Angeles, CA: Muthén \& Muthén.

Ning, H. K. \& Downing. K. (2015). A latent profile analysis of university students' selfregulated learning strategies. Studies in Higher Education, 40, 1328-1346. doi: 10.1080/03075079.2014.880832 
Nylund, K. L., Asparouhov, T., \& Muthén, B. O. (2007). Deciding on the number of classes in latent class analysis and growth mixture modeling: A Monte Carlo simulation study. Structural Equation Modeling: A Multidisciplinary Journal, 14, 535-569. doi: 10.1080/10705510701575396

Pastor, D. A., \& Gagné, P. (2013). Mean and covariance structure mixture models. In G. R. Hancock, \& R. O. Mueller (Eds.), Structural equation modeling: A second course (2nd ed., pp. 343-393). Charlotte, NC: Information Age.

Pennington, G. L., \& Roese, N. J. (2003). Regulatory focus and temporal distance. Journal of Experimental Social Psychology, 39, 563-576. doi: 10.1016/S0022 -1031(03)00058 -1

Perez, T., Cromley, J. G., \& Kaplan, A. (2014). The role of identity development, values, and costs in college STEM retention. Journal of Educational Psychology, 106, 315-329. doi: $10.1037 / \mathrm{a} 0034027$

Peugh, J., \& Fan, X. (2013). Modeling unobserved heterogeneity using latent profile analysis: A Monte Carlo simulation. Structural Equation Modeling: A Multidisciplinary Journal, 20, 616-639. doi: 10.1080/10705511.2013.824780

Pierro, A., Chernikova, M., Lo Destro, C., Higgins, E. T., \& Kruglanski, A. W. (2018). Assessment and locomotion conjunction: How looking complements leaping... But not always. In J. M. Olson (Ed.), Advances in experimental social psychology (Vol. 58, pp. 243-299). San Diego, CA: Academic Press. doi: 10.1016/bs.aesp.2018.02.001

Pierro, A., Giacomantonio, M., Pica, G., Kruglanski, A. W., \& Higgins, E. T. (2011). On the psychology of time in action: Regulatory mode orientations and procrastination. Journal of Personality and Social Psychology, 101, 1317-1331. doi: $10.1037 / \mathrm{a} 0025943$

Pierro, A., Giacomantonio, M., Pica, G., Kruglanski, A. W., \& Higgins, E. T. (2013). Locomotion and the preference for multi-tasking: Implications for well-being. Motivation and Emotion, 37, 213-223. doi: 10.1007/s11031-012-9300-y

Pierro, A., Kruglanski, A. W., \& Higgins, E. T. (2006). Regulatory mode and the joys of doing: Effects of 'locomotion' and 'assessment' on intrinsic and extrinsic task motivation. European Journal of Personality, 20, 355-375. 
doi: 10.1002/per.600

Pintrich, P. R. (1999). The role of motivation in promoting and sustaining self-regulated learning. International Journal of Educational Research, 31, 459-470. doi: 10.1016/S0883-0355(99)00015-4

Pintrich, P. R. (2004). A conceptual framework for assessing motivation and self-regulated learning in college students. Educational Psychology Review, 16, 385-407. doi: 10.1007/s10648-004-0006-x

Pintrich, P. R., \& De Groot, E. V. (1990). Motivational and self-regulated learning components of classroom academic performance. Journal of Educational Psychology, 82, 33-40. doi: 10.1037/0022-0663.82.1.33

Pintrich, P. R., Smith, D. A. F., Garcia, T., \& McKeachie, W. J. (1991). A manual for the use of the Motivated Strategies for Learning Questionnaire (MSLQ). Ann Arbor, MI: National Center for Research to Improve Postsecondary Teaching and Learning.

Pintrich, P. R., Smith, D. A., Garcia, T., \& McKeachie, W. J. (1993). Reliability and predictive validity of the Motivated Strategies for Learning Questionnaire (MSLQ). Educational and Psychological Measurement, 53, 801-813. doi: 10.1177/0013164493053003024

Raykov, T., \& Marcoulides, G. A. (2006). A first course in structural equation modeling (2nd ed.). Mahwah, NJ: Lawrence Erlbaum Associates.

Reeve, J., Ryan, R., Deci, E. L., \& Jang, H. (2008). Understanding and promoting autonomous self-regulation: A self-determination theory perspective. In D. H. Schunk, \& B. J. Zimmerman (Eds.), Motivation and self-regulated learning: Theory, research, and applications (pp. 223-244). New York, NY: Lawrence Erlbaum Associates.

Richardson, M., Abraham, C., \& Bond, R. (2012). Psychological correlates of university students' academic performance: A systematic review and meta-analysis. Psychological Bulletin, 138, 353-387. doi: 10.1037/a0026838

Robertson-Kraft, C., \& Duckworth, A. L. (2014). True grit: Trait-level perseverance and passion for long-term goals predicts effectiveness and retention among novice teachers. Teachers College Record, 116, 1-27.

Robson, C., (2011). Real world research: A resource for users of social research methods in applied settings (3rd ed.). Chichester, UK: Wiley. 
Rosenzweig, E. Q., \& Miele, D. B. (2016). Do you have an opportunity or an obligation to score well? The influence of regulatory focus on academic test performance. Learning and Individual Differences, 45, 114-127. doi: 10.1016/j.lindif.2015.12.005

Ryan, R. M., \& Deci, E. L. (2017). Self-determination theory: Basic psychological needs in motivation, development, and wellness. New York, NY: Guilford.

Ryan, R. M., \& Moller, A. C. (2017). Competence as a necessary but not sufficient condition for high quality motivation: A self-determination theory perspective. In A. J. Elliot, C. S. Dweck, \& D. S. Yeager, Handbook of competence and motivation (2nd ed., pp. 230-247). New York, NY: Guilford Press.

Saldaña, J. (2013). The coding manual for qualitative researchers (2nd ed.). Thousand Oaks, CA: Sage.

Scheel, M., Madabhushi, S., \& Backhaus, A. (2009). The academic motivation of at-risk students in a counseling prevention program. Counseling Psychologist, 37, 11471178. doi: 10.1177/0011000009338495

Scholer, A. A., \& Higgins, E. T. (2008). Distinguishing levels of approach and avoidance: An analysis using regulatory focus theory. In A. J. Elliot (Ed.), Handbook of approach and avoidance motivation (pp. 489-503). New York, NY: Psychology Press.

Scholer, A. A., \& Higgins, E. T. (2010). Conflict and control at different levels of selfregulation. In R. Hassin, K. Ochsner, \& Y. Trope (Eds.), Self-control in society, mind, and brain (pp. 312-334). New York, NY: Oxford University Press.

Scholer, A. A., \& Higgins, E. T. (2011). Promotion and prevention systems: Regulatory focus dynamics within self-regulatory hierachies. In K. Vohs \& R. Baumeister (Eds.), Handbook of self-regulation: Research, theory, and applications (2nd ed., pp. 143161). New York, NY: Guilford.

Scholer, A. A., \& Higgins, E. T. (2012). Too much of a good thing? Trade-offs in promotion and prevention focus. In R.M. Ryan (Ed.), The Oxford handbook of human motivation (pp. 65-84). New York, NY: Oxford University Press.

Scholer, A. A., Miele, D. B., Murayama, K., \& Fujita, K. (2018). New directions in selfregulation: The role of metamotivational beliefs. Current Directions in Psychological Science, 27, 437-442. doi: 10.1177/0963721418790549 
Schunk, D. H., Pintrich, P. R., Meece, J. L. (2008). Motivation in education: Theory, research, and applications (3rd ed.). Upper Saddle River, NJ: Pearson/Merrill Prentice Hall.

Schwarz, G. (1978). Estimating the dimension of a model. The Annals of Statistics, 6, 461-464. doi: 10.1007/BF02294360

Schwinger, M., Steinmayr, R., \& Spinath, B. (2009). How do motivational regulation strategies affect achievement: Mediated by effort management and moderated by intelligence? Learning and Individual Differences, 19, 621-627. doi: 10.1016/j.lindif.2009.08.006

Sclove, L. S. (1987). Application of model selection criteria to some problems in multivariate analysis. Psychometrika, 52, 333-343. doi: 10.1007/BF02294360

Seidman, I. (2013). Interviewing as qualitative research: A guide for researchers in education and the social sciences (4th ed.). New York, NY: Teachers College Press.

Senko, C., Hulleman, C. S., \& Harackiewicz, J. M. (2011). Achievement goal theory at the crossroads: Old controversies, current challenges, and new directions. Educational psychologist, 46, 26-47. doi: 10.1080/00461520.2011.538646

Simpkins, S. D., Davis-Kean, P., and Eccles, J. S. (2006). Math and science motivation: A longitudinal examination of the links between choices and beliefs, Developmental Psychology, 42, 70-83. doi: 10.1037/0012-1649.42.1.70

Shu, A. \& Lam, S. F. (2011). Are success and failure experiences equally motivational? An investigation of regulatory focus and feedback. Learning and Individual Differences, 21, 724-727. doi: 10.1016/j.lindif.2011.08.002

Shu, T. M, \& Lam, S. F. (2016). Is it always good to provide positive feedback to students? The moderating effects of culture and regulatory focus. Learning and Individual Differences, 49, 171-177. doi: 10.1016/j.lindif.2016.06.012

Soenens, B., \& Vansteenkiste, M. (2005). Antecedents and outcomes of self-determination in 3 life domains: The role of parents' and teachers' autonomy support. Journal of Youth and Adolescence, 34, 589-604. doi: 10.1007/s10964-005-8948-y

Sörbom, D. (1989). Model modification. Psychometrika, 54, 371-384. doi: 10.1007/BF02294623

Stake, R. E. (1995). The art of case study research. Thousand Oaks, CA: Sage. 
Stake, R. E. (2006). Multiple case study analysis. New York, NY: Guilford.

Steiger, J. H. (1990). Structural model evaluation and modification: An interval estimation approach. Multivariate Behavioral Research, 25, 173-180.

doi:10.1207/s15327906mbr2502_4

Steinmayr, R., Weidinger, A. F., \& Wigfield, A. (2018). Does students' grit predict their school achievement above and beyond their personality, motivation, and engagement? Contemporary Educational Psychology, 53, 106-122. doi: 10.1016/j.cedpsych.2018.02.004

Sungur, S. (2007). Modeling the relationships among students' motivational beliefs, metacognitive strategy use, and effort regulation. Scandinavian Journal of Educational Research, 51, 315-326. doi: 10.1080/00313830701356166

Tashakkori, A., \& Creswell, J. (2007). Editorial: The new era of mixed methods. Journal of Mixed Methods Research, 1, 3-7. doi: 10.1177/2345678906293042

Taylor, G., Jungert, T., Mageau, G. A., Schattke, K., Dedic, H., Rosenfield, S., \& Koestner, R. (2014). A self-determination theory approach to predicting school achievement over time: The unique role of intrinsic motivation. Contemporary Educational Psychology, 39, 342-358. doi: 10.1016/j.cedpsych.2014.08.002

Taylor, S. J., Bogdan, R., \& Devault, M. (2015). Introduction to qualitative research methods: A guidebook and resource. Hoboken, NJ: John Wiley \& Sons.

Teddlie, C., \& Yu, F. (2007). Mixed methods sampling a typology with examples. Journal of Mixed Methods Research, 1, 77-100. doi: 10.1177/2345678906292430

Tein, J-Y., Coxe, S., \& Cham, H. (2013). Statistical power to detect the correct number of classes in latent profile analysis. Structural Equation Modeling: A Multidisciplinary Journal, 20, 640-657. doi: 10.1080/10705511.2013.824781

Temple, B. \& Yong, A. (2004). Qualitative research and translation dilemmas. Qualitative Research, 4, 161-178. doi: 10.1177/1468794104044430

Tucker, L. R., \& Lewis, C. (1973). A reliability coefficient for maximum likelihood factor analysis. Psychometrika, 38, 1-10. doi:10.1007/BF02291170

Vaughn, L. A. (2017). Foundational tests of the need-support model: a framework for 
bridging regulatory focus theory and self-determination theory. Personality and Social Psychology Bulletin, 43, 313-328. doi: 10.1177/0146167216684132

Wang, J., \& Wang, X. (2012). Structural equation modeling: Applications using Mplus. Beijing, China: Higher Education Press.

Watt, H. M. G., Shapka, J. D., Morris, Z. A., Durik, A. M., Keating, D. P., \& Eccles, J. S. (2012). Gendered motivational processes affecting high school mathematics participation, educational aspirations, and career plans: A comparison of samples from Australia, Canada, and the United States. Developmental Psychology, 48, 1594-1611. doi: $10.1037 / \mathrm{a} 0027838$

Weiner, B. (1979). A theory of motivation for some classroom experiences. Journal of Educational Psychology, 71, 3-25. doi: 10.1037/0022-0663.71.1.3

Weiner, B. (1985). An attributional theory of achievement motivation and emotion. Psychological Review, 92, 548-573. doi: 10.1037/0033-295X.92.4.548

Weiner, B. (2010). The development of an attribution-based theory of motivation: A history of ideas. Educational Psychologist, 45, 28-36. doi: 10.1080/00461520903433596

West, R. F., Toplak, M. E., \& Stanovich, K. E. (2008). Heuristics and biases as measures of critical thinking: Associations with cognitive ability and thinking dispositions. Journal of Educational Psychology, 100, 930-941. doi: 10.1037/a0012842

Widaman, K. F., Little, T. D., Preacher, K. J., \& Sawalani, G. M. (2011). On creating and using short forms of scales in secondary research. In K. H. Trzesniewski, M. B. Donnellan, \& R. E. Lucas (Eds.), Secondary data analysis: An introduction for psychologists (pp. 39-61). Washington, DC: American Psychological Association.

Wigfield, A., \& Cambria, J. (2010). Students' achievement values, goal orientations, and interest: Definitions, development, and relations to achievement outcomes. Developmental Review, 30, 1-35. doi: 10.1016/j.dr.2009.12.001

Wigfield, A., \& Eccles, J. S. (2000). Expectancy-value theory of achievement motivation. Contemporary Educational Psychology, 25, 68-81. doi: 10.1006/ceps.1999.1015

Wigfield, A., Muenks, K., \& Rosenzweig, E. Q. (2015). Children's achievement motivation in school. In C. M. Rubie-Davies, J. M. Stephenson, \& P. Watson (Eds.), Routledge iternational handbook of social psychology of the classroom (pp. 9-20). New York, 
NY: Routledge.

Wigfield, A., Tonks, S., \& Klauda, S. L. (2009). Expectancy-value theory. In K. A. Wentzel \& A. Wigfield (Eds.), Handbook of motivation at school (pp. 55-75). New York, NY: Routledge.

Yeager, D. S. \& Dweck, C. S. (2012). Mindsets that promote resilience: When students believe that personal characteristics can be developed. Educational Psychologist, 47, 302-314. doi: $10.1080 / 00461520.2012 .722805$

Yin, R.K. (2009). Case study research: Design and methods (4th ed.), Thousand Oaks, CA: Sage.

Yip, M.C.W. (2007). Differences in learning and study strategies between high and low achieving university students: A Hong Kong study. Educational Psychology 27, 597606. doi: 10.1080/01443410701309126

Zhang, J. (2016). Can MOOCs be interesting to students? An experimental investigation from regulatory focus perspective. Computers \& Education, 95, 340-351. doi:10.1016/j.compedu.2016.02.003

Zhang, S., Shi, R., Yun, L., Li, X., Wang, Y., He, H., \& Miao, D. (2015). Self-regulation and study-related health outcomes: A structural equation model of regulatory mode orientations, academic burnout and engagement among university students. Social Indicators Research, 123, 585-599. doi: 10.1007/s11205-014-0742-3

Ziegler, N., \& Opdenakker, M. C. (2018). The development of academic procrastination in first-year secondary education students: The link with metacognitive self-regulation, self-efficacy, and effort regulation. Learning and Individual Differences, 64, 71-82. doi: 10.1016/j.lindif.2018.04.009

Zimmerman, B. J., \& D. H. Schunk. (2008). Motivation: An essential dimension of selfregulated learning. In D. H. Schunk, \& B. J. Zimmerman (Eds.), Motivation and selfregulated learning: Theory, research, and applications (pp. 1-30). New York, NY: Lawrence Erlbaum Associates. 


\title{
Appendices
}

\section{Appendix A: Information Sheet for the Questionnaire Participants}

\author{
TE WHARE WĀNANGA O TE ŨPOKO O TE IKA A MĀUI

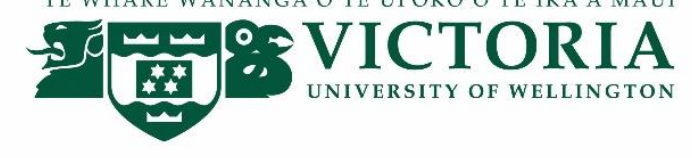 \\ CHINESE UNDERGRADUATE STUDENTS'MOTIVATION \\ PROFILES: \\ IDENTIFICATION AND SIGNIFICANCE
}

INFORMATION SHEET FOR SURVEY PARTICIPANTS

Thank you for your interest in this project. Please read this information before deciding whether or not to take part. If you decide to participate, thank you. If you decide not to take part, thank you for considering my request.

\section{Who am I?}

My name is Jie Gao and I am a Doctoral student in Faculty of Education at Victoria University of Wellington. This research project is work towards my thesis.

\section{What is the aim of the project?}

Motivation is related to important aspects of students' learning experiences. This research aims to identify the motivation profiles of Chinese undergraduate students and establish the motivational significance of each profile identified. This information will contribute to the existing literature on motivation and motivation effects. In addition, it could provide potentially important information regarding the motivation factors supporting academic success of Chinese undergraduate students. This research has been approved by the Victoria University of Wellington Human Ethics Committee [No. 24379]. Consent to carry out this 
research has been granted by the Head of School/Department and the course lecturer.

\section{How can you help?}

To participate in this study, you will need to be (a) enrolled in full time undergraduate programmes at a university in mainland China; and (b) born and partially educated in mainland China. The survey takes approximately 20 minutes to complete and includes questions regarding your motivation. There will be an opportunity to participate in an online interview about your motivation and learning experiences after the survey. If you are willing to be interviewed, you will be provided with a further information sheet relating specifically to the interview. Your decision to participate in this research project is completely voluntary. Submission of this survey implies consent for participating in the research. There will be no consequences whatsoever associated with your decision not to participate in this study.

\section{What will happen to the information you give?}

Participation in this research is anonymous unless you agree to be contacted for a follow-up online interview. In case you agree to be contacted with regard to the interview, this research is confidential. This means that my supervisors and I will be aware of your identity but the research data will be aggregated and your identity will not be disclosed in any reports, presentations, or presentations associated with this research. If you agree to participate in the interview and are selected to do so, your name will not be used in any reports, publications, or presentations; a pseudonym will be used instead. All material will be kept secure and confidential. Only my supervisors and I will have access to the data from this research. All data collected will be destroyed seven years after the research ends.

\section{What will the project produce?}

The information from my research will be used in my $\mathrm{PhD}$ thesis and for publication in academic journals, book chapters, or presentations at conferences.

\section{If you accept this invitation, what are your rights as a research participant?}


You do not have to accept this invitation if you don't want to. If you do decide to participate, you have the right to:

- choose not to answer any question;

- $\quad$ ask any questions about the study at any time;

- be able to read a summary report of findings from this research by emailing the researcher to request a copy.

\section{If you have any questions or problems, who can you contact?}

If you have any questions, either now or in the future, please feel free to contact either:

\section{Student:}

Name: Jie Gao

University email address:

Jie.Gao@vuw.ac.nz

\section{Supervisors:}

Name: Dr. Flaviu-Adrian Hodis Name: Dr. Carolyn Frances Tait

Role: primary supervisor

School: Education

Phone: + 64-4-463-9550

flaviu.hodis@vuw.ac.nz
Role: secondary supervisor

School: Education

Phone: +64-4-463-9590

Carolyn.Tait@vuw.ac.nz

\section{Human Ethics Committee information}

If you have any concerns about the ethical conduct of the research you may contact the Victoria University HEC Convener: Associate Professor Susan Corbett. Email susan.corbett@vuw.ac.nz or telephone +64-4-463 5480. 


\section{Appendix B: Modifications of the Questionnaire Items}

\begin{tabular}{|c|c|c|c|}
\hline Number & Acronym & Original version & Revised version \\
\hline Q1 & H_PRO_5 & $\begin{array}{l}\text { I feel like I have made progress toward being successful } \\
\text { in my life. }\end{array}$ & \\
\hline Q2 & K_LOCO_1 & $\begin{array}{l}\text { I don't mind doing things even if they involve extra } \\
\text { effort. }\end{array}$ & \\
\hline Q3 & DW_EN_1 & $\begin{array}{l}\text { You have a certain amount of intelligence, and you can't } \\
\text { really do much to change it. }\end{array}$ & $\begin{array}{l}\text { I have a certain amount of intelligence, and I can't } \\
\text { really do much to change it. }\end{array}$ \\
\hline Q4 & H_PRE_2 & $\begin{array}{l}\text { Did you get on your parents' nerves often when you } \\
\text { were growing up? }\end{array}$ & When I grew up, I often annoyed my parents \\
\hline Q5 & H_PRO_1 & $\begin{array}{l}\text { Compared to most people, are you typically unable to } \\
\text { get what you want out of life? }\end{array}$ & $\begin{array}{l}\text { Compared to most people, I am typically able to get } \\
\text { what I want out of life. }\end{array}$ \\
\hline Q6 & K_LOCO_2 & I am a "workaholic". & \\
\hline Q7 & K_ASSE_3 & I like evaluating other people's plans. & I often evaluate other people's plans. \\
\hline Q8 & DU_G_S_C_1 & $\begin{array}{l}\text { I often set a goal but later choose to pursue a different } \\
\text { one. }\end{array}$ & \\
\hline Q9 & P_CRT_1 & $\begin{array}{l}\text { I often find myself questioning things I hear or read in } \\
\text { this course to decide if I find them convincing. }\end{array}$ & $\begin{array}{l}\text { I often find myself questioning things I hear or read in } \\
\text { my classes to decide if I find them convincing. }\end{array}$ \\
\hline
\end{tabular}




\begin{tabular}{|c|c|c|c|}
\hline Number & Acronym & Original version & Revised version \\
\hline Q10 & DW_IN_4 & $\begin{array}{l}\text { You can change even your basic intelligence level } \\
\text { considerably. }\end{array}$ & $\begin{array}{l}\text { I can change even my basic intelligence level } \\
\text { considerably. }\end{array}$ \\
\hline Q11 & H_PRO_6 & $\begin{array}{l}\text { I have found very few hobbies or activities in my life } \\
\text { that capture my interest or motivate me to put effort into } \\
\text { them. }\end{array}$ & $\begin{array}{l}\text { There are activities in my life that capture my interest } \\
\text { or motivate me to put effort into them. }\end{array}$ \\
\hline Q12 & K_LOCO_3 & I feel excited just before I am about to reach a goal. & \\
\hline Q13 & K_ASSE_5 & $\begin{array}{l}\text { I don't spend much time thinking about ways others } \\
\text { could improve themselves. }\end{array}$ & $\begin{array}{l}\text { I spend a lot of time thinking about ways others could } \\
\text { improve themselves. }\end{array}$ \\
\hline Q14 & K_LOCO_4 & $\begin{array}{l}\text { I enjoy actively doing things, more than just watching } \\
\text { and observing. }\end{array}$ & \\
\hline Q15 & H_PRE_1 & $\begin{array}{l}\text { Growing up, would you ever "cross the line" by doing } \\
\text { things that your parents would not tolerate? }\end{array}$ & $\begin{array}{l}\text { Growing up, I often "crossed the line" by doing things } \\
\text { that my parents would not tolerate. }\end{array}$ \\
\hline Q16 & K_ASSE_1 & $\begin{array}{l}\text { I never evaluate my social interactions with others after } \\
\text { they occur. }\end{array}$ & $\begin{array}{l}\text { I usually evaluate my social interactions with others } \\
\text { after they occur. }\end{array}$ \\
\hline Q17 & DW_IN_1 & $\begin{array}{l}\text { No matter who you are, you can significantly change } \\
\text { your intelligence level. }\end{array}$ & $\begin{array}{l}\text { No matter who I am, I can significantly change my } \\
\text { intelligence level. }\end{array}$ \\
\hline Q18 & K_LOCO_7 & $\begin{array}{l}\text { When I decide to do something, I can't wait to get } \\
\text { started. }\end{array}$ & \\
\hline
\end{tabular}




\begin{tabular}{|c|c|c|c|}
\hline Number & Acronym & Original version & Revised version \\
\hline Q19 & H_PRO_4 & $\begin{array}{l}\text { When it comes to achieving things that are important to } \\
\text { me, I find that I don't perform as well as I ideally would } \\
\text { like to do. }\end{array}$ & $\begin{array}{l}\text { When it comes to achieving things that are important } \\
\text { to me, I find that I perform as well as I ideally would } \\
\text { like to do. }\end{array}$ \\
\hline Q20 & K_LOCO_9 & I am a "low energy" person. & I usually have a lot of energy. \\
\hline Q21 & H_PRE_3 & $\begin{array}{l}\text { How often did you obey rules and regulations that were } \\
\text { established by your parents? }\end{array}$ & $\begin{array}{l}\text { I usually obeyed rules and regulations that were } \\
\text { established by my parents. }\end{array}$ \\
\hline Q22 & K_LOCO_11 & $\begin{array}{l}\text { When I get started on something, I usually persevere } \\
\text { until I finish it. }\end{array}$ & \\
\hline Q23 & K_ASSE_6 & I often critique work done by myself or others. & $\begin{array}{l}\text { I often evaluate critically work done by myself or } \\
\text { others. }\end{array}$ \\
\hline Q24 & P_ERE_2 & $\begin{array}{l}\text { I work hard to do well in this class even if I don't like } \\
\text { what we are doing. }\end{array}$ & $\begin{array}{l}\text { I work hard to do well in my classes even if I don't } \\
\text { like what we are doing. }\end{array}$ \\
\hline Q25 & DW_EN_3 & $\begin{array}{l}\text { To be honest, you can't really change how intelligent } \\
\text { you are. }\end{array}$ & $\begin{array}{l}\text { To be honest, I can't really change how intelligent I } \\
\text { am. }\end{array}$ \\
\hline Q26 & K_LOCO_5 & I am a "doer". & \\
\hline Q27 & K_ASSE_4 & I often compare myself with other people. & \\
\hline Q28 & H_PRE_5 & $\begin{array}{l}\text { Not being careful enough has gotten me into trouble at } \\
\text { times. }\end{array}$ & \\
\hline Q29 & K_ASSE_7 & I often feel that I am being evaluated by others. & \\
\hline
\end{tabular}




\begin{tabular}{|c|c|c|c|}
\hline Number & Acronym & Original version & Revised version \\
\hline Q31 & DW_EN_4 & $\begin{array}{l}\text { You can learn new things, but you can't really change } \\
\text { your basic intelligence. }\end{array}$ & $\begin{array}{l}\text { I can learn new things, but I can't really change my } \\
\text { basic intelligence. }\end{array}$ \\
\hline Q32 & P_CRT_5 & $\begin{array}{l}\text { Whenever I read or hear an assertion or conclusion in } \\
\text { this class, I think about possible alternatives. }\end{array}$ & $\begin{array}{l}\text { Whenever I read or hear an assertion (观点) or } \\
\text { conclusion in my classes, I think about possible } \\
\text { alternatives. }\end{array}$ \\
\hline Q33 & H_PRE_4 & $\begin{array}{l}\text { Growing up, did you ever act in ways that your parents } \\
\text { thought were objectionable? }\end{array}$ & $\begin{array}{l}\text { Growing up, I often acted in ways that my parents } \\
\text { thought were objectionable. }\end{array}$ \\
\hline Q34 & DU_G_S_P_2 & Setbacks don’t discourage me. & \\
\hline Q35 & K_ASSE_12 & $\begin{array}{l}\text { When I meet a new person I usually evaluate how well } \\
\text { he or she is doing on various dimensions (e.g., looks, } \\
\text { achievements, social status, clothes). }\end{array}$ & \\
\hline Q36 & K_LOCO_10 & $\begin{array}{l}\text { Most of the time my thoughts are occupied with the task } \\
\text { I wish to accomplish. }\end{array}$ & \\
\hline Q37 & K_ASSE_2 & $\begin{array}{l}\text { I spend a great deal of time taking inventory of my } \\
\text { positive and negative characteristics. }\end{array}$ & $\begin{array}{l}\text { I spend a lot of time evaluating my positive and } \\
\text { negative characteristics. }\end{array}$ \\
\hline
\end{tabular}




\begin{tabular}{|c|c|c|c|}
\hline Number & Acronym & Original version & Revised version \\
\hline Q38 & P_ERE_4 & $\begin{array}{l}\text { Even when course materials are dull and uninteresting, I } \\
\text { manage to keep working until I finish. }\end{array}$ & \\
\hline Q39 & DU_G_S_P_3 & I am a hard worker. & \\
\hline Q40 & K_ASSE_11 & $\begin{array}{l}\text { I rarely analyze the conversations I have had with others } \\
\text { after they occur. }\end{array}$ & $\begin{array}{l}\text { I usually analyze the conversations I have had with } \\
\text { others after they occur. }\end{array}$ \\
\hline Q41 & P_CRT_3 & $\begin{array}{l}\text { I treat the course material as a starting point and try to } \\
\text { develop my own ideas about it. }\end{array}$ & \\
\hline Q42 & DU_G_S_C_4 & $\begin{array}{l}\text { I have difficulty maintaining my focus on projects that } \\
\text { take more than a few months to complete. }\end{array}$ & \\
\hline Q43 & DU_G_S_P_4 & I am diligent. & \\
\hline Q44 & P_CRT_2 & $\begin{array}{l}\text { When a theory, interpretation, or conclusion is presented } \\
\text { in class or in the readings, I try to decide if there is good } \\
\text { supporting evidence. }\end{array}$ & \\
\hline Q45 & H_PRO_3 & Do you often do well at different things that you try? & I often do well at different things that I try. \\
\hline Q46 & DW_EN_2 & $\begin{array}{l}\text { Your intelligence is something about you that you can't } \\
\text { change very much. }\end{array}$ & $\begin{array}{l}\text { My intelligence is something about me that I can't } \\
\text { change very much. }\end{array}$ \\
\hline Q47 & H_PRO_2 & $\begin{array}{l}\text { How often have you accomplished things that got you } \\
\text { "psyched" to work even harder? }\end{array}$ & $\begin{array}{l}\text { Accomplishing things motivates me to work even } \\
\text { harder. }\end{array}$ \\
\hline
\end{tabular}




\begin{tabular}{|c|c|c|c|}
\hline Number & Acronym & Original version & Revised version \\
\hline Q48 & DU_G_S_P_1 & I finish whatever I begin. & \\
\hline Q49 & P_ERE_1 & $\begin{array}{l}\text { I often find myself questioning things I hear or read in } \\
\text { this course to decide if I find them convincing. }\end{array}$ & $\begin{array}{l}\text { I often feel so lazy or bored when I study for my } \\
\text { classes that I quit before I finish what I planned to do. }\end{array}$ \\
\hline Q50 & K_LOCO_8 & $\begin{array}{l}\text { By the time I accomplish a task, I already have the next } \\
\text { one in mind. }\end{array}$ & \\
\hline Q51 & K_ASSE_10 & $\begin{array}{l}\text { I often think that other people's choices and decisions } \\
\text { are wrong. }\end{array}$ & I often evaluate other people's choices and decisions. \\
\hline Q52 & P_ERE_3 & $\begin{array}{l}\text { When course work is difficult, I gave up or only study } \\
\text { the easy parts. }\end{array}$ & \\
\hline Q53 & DW_IN_2 & $\begin{array}{l}\text { You can always substantially change how intelligent you } \\
\text { are. }\end{array}$ & $\begin{array}{l}\text { I can always substantially change how intelligent I } \\
\text { am. }\end{array}$ \\
\hline Q54 & P_CRT_4 & $\begin{array}{l}\text { I try to play around with ideas of my own related to } \\
\text { what I am learning in this course. }\end{array}$ & $\begin{array}{l}\text { I try to develop ideas of my own that are related to } \\
\text { what I learn in my classes. }\end{array}$ \\
\hline Q55 & K_LOCO_12 & I am a "go-getter". & I am a “go-getter" (乐于行动的人). \\
\hline Q56 & DU_G_S_C_3 & $\begin{array}{l}\text { I have been obsessed with a certain idea or project for a } \\
\text { short time but later lost interest. }\end{array}$ & \\
\hline Q57 & K_ASSE_8 & I am a critical person. & \\
\hline
\end{tabular}




\begin{tabular}{lcll}
\hline Number & Acronym & \multicolumn{1}{c}{ Original version } & \multicolumn{1}{c}{ Revised version } \\
\hline Q58 & K_LOCO_6 & $\begin{array}{l}\text { When I finish one project, I often wait awhile before } \\
\text { getting started on a new one. }\end{array}$ & $\begin{array}{l}\text { When I finish one project, I immediately start a new } \\
\text { one. }\end{array}$ \\
Q59 & K_ASSE_9 & $\begin{array}{l}\text { I am very self-critical and self-conscious about what I } \\
\text { am saying. }\end{array}$ & $\begin{array}{l}\text { I am very self-critical and self-conscious (谨慎)about } \\
\text { what I am saying. }\end{array}$ \\
Q60 & DW_IN_3 & $\begin{array}{l}\text { No matter how much intelligence you have, you can } \\
\text { always change it quite a bit. }\end{array}$ & $\begin{array}{l}\text { No matter how much intelligence I have, I can always } \\
\text { change it quite a bit. }\end{array}$ \\
\hline
\end{tabular}

Note. 1. Each number denotes the position of the item in the original instrument. For example, H_PRO_1 denotes the first promotion indicator in the Regulatory Focus Questionnaire scale (RFQ; Higgins et al., 2001).

2. Meanings of the acronyms.

H_PRO_5 = The fifth promotion indicator in the RFQ scale (Higgins et al., 2001).

K_LOCO_1 = The first locomotion indicator in the Regulatory Mode Questionnaire scale (RMQ; Kruglanski et al., 2000).

DW_EN_1 = The first item in the subscale of Entity Theory in the Theories of Intelligence Scale (Dweck, 1999).

H_PRE_2 = The second prevention indicator in the RFQ scale (Higgins et al., 2001).

K_ASSE_3 = The third assessment indicator in the RMQ scale (Kruglanski et al., 2000).

DU_G_S_C_1 = The first indicator of the subscale of consistency of interest in the Short Grit Scale (Grit-S; Duckworth \& Quinn, 2009).

P_CRT_1 = The first indicator of critical thinking in the Critical Thinking Scale of the Motivated Strategies for Learning Questionnaire (MSLQ; Pintrich et al., 1991).

DW_IN_4 = The fourth item in the subscale of Incremental Theory in the Theories of Intelligence Scale (Dweck, 1999).

P_ERE_2 = The second indicator of effort regulation in the Effort Regulation Scale of MSLQ (Pintrich et al., 1991). 
3. Item composition of the scales.

H_PRO items: Q5; Q47; Q45; Q19; Q1; Q11

H_PRE items: Q15; Q4; Q21; Q33; Q28

K_LOCO items: Q2; Q6; Q12; Q14; Q26; Q58; Q18; Q50; Q20; Q36; Q22; Q55

K_ASSE items: Q16; Q37; Q7; Q27; Q13; Q23; Q29; Q57; Q59; Q51; Q40; Q35

DW_EN items: Q3; Q46; Q25; Q31

DW_IN items: Q17; Q53; Q60; Q10

DU_G_S_C items: Q8; Q30; Q56; Q42

DU_G_S_P items: Q48; Q34; Q39; Q43

P_CRT items: Q9; Q44; Q41; Q54; Q32

P_ERE items: Q49; Q24; Q52; Q38 


\section{Appendix C: Questionnaire}

\section{What motivates you to study?}

This survey aims to assist the researcher in gaining a better understanding of your motivation and studies. Please read it carefully and give your answer sincerely. The information will be kept entirely confidential. Thank you very much for your help! 亲爱的同学, 欢迎参加本次问卷调查! 本次调查将关注您的学习动机以及学习情况。请认真阅 读问卷的内容, 根据自身实际情况和真实感受如实填写。您所填写的所有信息都会得到严格保密。

\section{Part One: Your motivation.}

Please indicate how much you agree or disagree with each of the following statements by simply circling the corresponding number for each question.

请选择并圈出一个合适的数字，表明您对该陈述的认可程度。

1. I feel like I have made progress toward being successful in my life.

2. I don't mind doing things even if they involve extra effort.

$\begin{array}{ll}\text { strongly } & \text { strongly } \\ \text { disagree } & \text { agree }\end{array}$

$\begin{array}{lllllll}1 & 2 & 3 & 4 & 5 & 6 & 7\end{array}$

$\begin{array}{lllllll}1 & 2 & 3 & 4 & 5 & 6 & 7\end{array}$

3. I have a certain amount of intelligence, and I can't really do much to change it.

4. When I grew up, I often annoyed my parents

5. Compared to most people, I am typically able to get what I want out of life.

\section{$\begin{array}{lllllll}1 & 2 & 3 & 4 & 5 & 6 & 7\end{array}$}

$\begin{array}{lllllll}1 & 2 & 3 & 4 & 5 & 6 & 7\end{array}$

$\begin{array}{lllllll}1 & 2 & 3 & 4 & 5 & 6 & 7\end{array}$

6. I am a "workaholic".

$\begin{array}{lllllll}1 & 2 & 3 & 4 & 5 & 6 & 7\end{array}$

7. I often evaluate other people's plans.

$\begin{array}{lllllll}1 & 2 & 3 & 4 & 5 & 6 & 7\end{array}$

8. I often set a goal but later choose to pursue a different one.

$\begin{array}{lllllll}1 & 2 & 3 & 4 & 5 & 6 & 7\end{array}$

9. I often find myself questioning things I hear or read in my classes to decide if I find them convincing.

$\begin{array}{lllllll}1 & 2 & 3 & 4 & 5 & 6 & 7\end{array}$

10. I can change even my basic intelligence level considerably. $\begin{array}{lllllll}1 & 2 & 3 & 4 & 5 & 6 & 7\end{array}$ 


\section{strongly \\ disagree \\ strongly \\ agree}

11. There are activities in my life that capture my interest or motivate me to put effort into them.

12. I feel excited just before I am about to reach a goal.

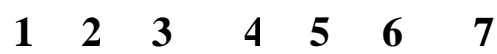

13. I spend a lot of time thinking about ways others could improve themselves.

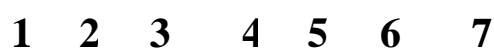

14. I enjoy actively doing things, more than just watching and observing.

15. Growing up, I often "crossed the line" by doing things that my parents would not tolerate.

16. I usually evaluate my social interactions with others after they occur.

$\begin{array}{lllllll}1 & 2 & 3 & 4 & 5 & 6 & 7\end{array}$

$\begin{array}{lllllll}1 & 2 & 3 & 4 & 5 & 6 & 7\end{array}$

$\begin{array}{lllllll}1 & 2 & 3 & 4 & 5 & 6 & 7\end{array}$

17. No matter who I am, I can significantly change my intelligence level.

18. When I decide to do something, I can't wait to get started.

19. When it comes to achieving things that are important to me, I find that I perform as well as I ideally would like to do.

$\begin{array}{lllllll}1 & 2 & 3 & 4 & 5 & 6 & 7\end{array}$

$\begin{array}{lllllll}1 & 2 & 3 & 4 & 5 & 6 & 7\end{array}$

$\begin{array}{lllllll}1 & 2 & 3 & 4 & 5 & 6 & 7\end{array}$

20. I usually have a lot of energy.

$\begin{array}{lllllll}1 & 2 & 3 & 4 & 5 & 6 & 7\end{array}$

21. I usually obeyed rules and regulations that were established by my parents.

$\begin{array}{lllllll}1 & 2 & 3 & 4 & 5 & 6 & 7\end{array}$

22. When I get started on something, I usually persevere until I finish it.

$\begin{array}{lllllll}1 & 2 & 3 & 4 & 5 & 6 & 7\end{array}$

$\begin{array}{lllllllll}\text { 23. I often evaluate critically work done by myself or others. } & 1 & \mathbf{2} & \mathbf{3} & \mathbf{4} & \mathbf{5} & \mathbf{6} & \mathbf{7}\end{array}$

24. I work hard to do well in my classes even if I don't like what we are doing.

$\begin{array}{lllllll}1 & 2 & 3 & 4 & 5 & 6 & 7\end{array}$

25. To be honest, I can’t really change how intelligent I am. $\quad \begin{array}{lllllll}\mathbf{1} & \mathbf{2} & \mathbf{3} & \mathbf{4} & \mathbf{5} & \mathbf{6} & \mathbf{7}\end{array}$

26. I am a "doer".

$\begin{array}{lllllll}1 & 2 & 3 & 4 & 5 & 6 & 7\end{array}$

27. I often compare myself with other people.

$\begin{array}{lllllll}1 & 2 & 3 & 4 & 5 & 6 & 7\end{array}$ 


$\begin{array}{ll}\text { strongly } & \text { strongly } \\ \text { disagree } & \text { agree }\end{array}$

28. Not being careful enough has gotten me into trouble at times.

29. I often feel that I am being evaluated by others.

30. New ideas and projects sometimes distract me from previous ones.

31. I can learn new things, but I can't really change my basic intelligence.

32. Whenever I read or hear an assertion (观点) or conclusion in my classes, I think about possible alternatives.

33. Growing up, I often acted in ways that my parents thought were objectionable.

34. Setbacks don't discourage me.

35. When I meet a new person I usually evaluate how well he or she is doing on various dimensions (e.g., looks, achievements, social status, clothes).

36. Most of the time my thoughts are occupied with the task I wish to accomplish.

37. I spend a lot of time evaluating my positive and negative characteristics.

38. Even when course materials are dull and uninteresting, I manage to keep working until I finish.

39. I am a hard worker.

40. I usually analyze the conversations I have had with others after they occur.

41. I treat the course material as a starting point and try to develop my own ideas about it.

42. I have difficulty maintaining my focus on projects that take more than a few months to complete.

43. I am diligent. $\begin{array}{lllllll}1 & 2 & 3 & 4 & 5 & 6 & 7\end{array}$

$\begin{array}{lllllll}1 & 2 & 3 & 4 & 5 & 6 & 7\end{array}$

$\begin{array}{lllllll}1 & 2 & 3 & 4 & 5 & 6 & 7\end{array}$

$\begin{array}{lllllll}1 & 2 & 3 & 4 & 5 & 6 & 7\end{array}$

$\begin{array}{lllllll}1 & 2 & 3 & 4 & 5 & 6 & 7\end{array}$

$\begin{array}{lllllll}1 & 2 & 3 & 4 & 5 & 6 & 7\end{array}$

$\begin{array}{lllllll}1 & 2 & 3 & 4 & 5 & 6 & 7\end{array}$

$\begin{array}{lllllll}1 & 2 & 3 & 4 & 5 & 6 & 7\end{array}$

$\begin{array}{lllllll}1 & 2 & 3 & 4 & 5 & 6 & 7\end{array}$

$\begin{array}{lllllll}1 & 2 & 3 & 4 & 5 & 6 & 7\end{array}$

$\begin{array}{lllllll}1 & 2 & 3 & 4 & 5 & 6 & 7\end{array}$

$\begin{array}{lllllll}1 & 2 & 3 & 4 & 5 & 6 & 7\end{array}$

$\begin{array}{lllllll}1 & 2 & 3 & 4 & 5 & 6 & 7\end{array}$

$\begin{array}{lllllll}1 & 2 & 3 & 4 & 5 & 6 & 7\end{array}$

$\begin{array}{lllllll}1 & 2 & 3 & 4 & 5 & 6 & 7\end{array}$

$\begin{array}{lllllll}1 & 2 & 3 & 4 & 5 & 6 & 7\end{array}$ 


$\begin{array}{ll}\text { strongly } & \text { strongly } \\ \text { disagree } & \text { agree }\end{array}$

44. When a theory, interpretation, or conclusion is presented in class or in the readings, I try to decide if there is good $\begin{array}{lllllll}1 & 2 & 3 & 4 & 5 & 6 & 7\end{array}$ supporting evidence.

45. I often do well at different things that I try.

$\begin{array}{lllllll}1 & 2 & 3 & 4 & 5 & 6 & 7\end{array}$

46. My intelligence is something about me that I can't change very much.

$\begin{array}{lllllll}1 & 2 & 3 & 4 & 5 & 6 & 7\end{array}$

47. Accomplishing things motivates me to work even harder.

48. I finish whatever I begin.

$\begin{array}{lllllll}1 & 2 & 3 & 4 & 5 & 6 & 7\end{array}$

49. I often feel so lazy or bored when I study for my classes that I quit before I finish what I planned to do.

$\begin{array}{lllllll}1 & 2 & 3 & 4 & 5 & 6 & 7\end{array}$

50. By the time I accomplish a task, I already have the next one in mind.

51. I often evaluate other people's choices and decisions.

$\begin{array}{lllllll}1 & 2 & 3 & 4 & 5 & 6 & 7\end{array}$

52. When course work is difficult, I gave up or only study the easy parts.

$\begin{array}{lllllll}1 & 2 & 3 & 4 & 5 & 6 & 7\end{array}$

53. I can always substantially change how intelligent I am.

$\begin{array}{lllllll}1 & 2 & 3 & 4 & 5 & 6 & 7\end{array}$

54. I try to develop ideas of my own that are related to what I learn in my classes.

$\begin{array}{lllllll}1 & 2 & 3 & 4 & 5 & 6 & 7\end{array}$

55. I am a “go-getter” (乐于行动的人).

$\begin{array}{lllllll}1 & 2 & 3 & 4 & 5 & 6 & 7\end{array}$

56. I have been obsessed with a certain idea or project for a short time but later lost interest.

57. I am a critical person.

$\begin{array}{lllllll}1 & 2 & 3 & 4 & 5 & 6 & 7\end{array}$

$\begin{array}{lllllll}1 & 2 & 3 & 4 & 5 & 6 & 7\end{array}$

58. When I finish one project, I immediately start a new one. $\begin{array}{llllllll} & \mathbf{1} & \mathbf{2} & \mathbf{3} & \mathbf{4} & \mathbf{5} & \mathbf{6} & \mathbf{7}\end{array}$

59. I am very self-critical and self-conscious (谨慎)about what I am saying.

$\begin{array}{lllllll}1 & 2 & 3 & 4 & 5 & 6 & 7\end{array}$

60. No matter how much intelligence I have, I can always change it quite a bit. 


\section{Part Two. Descriptive information about you.}

请填写以下个人信息。

1. Gender: $\square \mathrm{M} \quad \square \mathrm{F} \quad \square$ Other

2. Major:

3. How long have you been studying in your degree programme at this university?

$\square$ Less than a year

$\square$ Between one and two years

$\square$ Between two and three years

$\square$ More than three years

4. How do you evaluate your success with your studies so far at university? On a scale from 0 to 100 , where 0 is "not at all successful" and 100 is "extremely successful", please rate your success with your studies at the university so far (write here the number between 0 and 100 that reflects best your rating).

Part Three. Please answer the following short puzzles by writing your answer in the space provided.

请完成下面的脑筋急转弯, 并把答案写在划线处。

1. A bat and a ball cost $\$ 1.10$ in total. The bat costs $\$ 1.00$ more than the ball. How much does the ball cost? cents

2. If it takes 5 machines to make 5 phones, how long would it take 100 machines to make 100 phones? minutes

3. In a lake, there is a patch of lily pads. Every day, the patch doubles in size. If it takes 48 days for the patch to cover the entire lake, how long would it take for the patch of to cover half of the lake? days

\section{Thank you again for taking your time to compete this survey!}

This research includes a second phase in which I will interview some students regarding their motivation. If you are interested to get some more information about the interview and are considering participation, please leave your contact details below. 
再次感谢您的参与!

本研究还包括第二阶段的访谈部分。我将邀请部分同学针对他们的学习动机进行访谈。如果您想了 解访谈的更多相关信息，并考虑参加接下来的访谈，请留下您的姓名和联系方式。

Name:

Email:

Phone number:

QQ or Wechat ID: 


\section{Appendix D: Information Sheet for Interview Participants}

\section{CHINESE UNDERGRADUATE STUDENTS' MOTIVATION PROFILES:

\author{
IDENTIFICATION AND SIGNIFICANCE
} INFORMATION SHEET FOR INTERVIEW PARTICIPANTS}

Thank you for your interest in this project. Please read this information before deciding whether or not to take part. If you decide to participate, thank you. If you decide not to take part, thank you for considering my request.

\section{Who am I?}

My name is Jie Gao and I am a Doctoral student in Faculty of Education at Victoria University of Wellington. This research project is work towards my thesis.

\section{What is the aim of the project?}

Motivation is related to important aspects of students' learning experiences. This research aims to identify the motivation profiles of Chinese undergraduate students and establish the motivational significance of each profile identified. This information will contribute to the existing literature on motivation and motivation effects. In addition, it could provide potentially important information regarding the motivation factors supporting academic success of Chinese undergraduate students. This research has been approved by the Victoria University of Wellington Human Ethics Committee [No. 24379]. 


\section{How can you help?}

If you agree to take part and you are selected to participate, I will interview you online via Wechat or QQ. I will ask you questions about your motivation and learning experiences. The interview will take approximately 45 minutes. I will audio record the interview and transcribe it later. You can stop the interview at any time, without giving a reason. I will return the transcript of the interview to you and you can make comments, add clarifications, and/or suggest changes. You can withdraw from the study by contacting me at any point before January 15, 2018. If you withdraw, the information you provided will be destroyed.

\section{What will happen to the information you give?}

This research is confidential. This means that the researcher will be aware of your identity but the research data will be aggregated and your identity will not be disclosed in any reports, publications, or presentations. Only my supervisors and I will read the notes or transcript of the interview. The interview transcripts, summaries and any recordings will be kept securely and destroyed seven years after the research ends.

\section{What will the project produce?}

The information from my research will be used in my $\mathrm{PhD}$ thesis and for publication in academic journals, book chapters, or presentations at conferences.

\section{If you accept this invitation, what are your rights as a research participant?}

You do not have to accept this invitation if you don't want to. If you do decide to participate, you have the right to:

- choose not to answer any question;

- $\quad$ ask for the recorder to be turned off at any time during the interview;

- withdraw from the study before January 15, 2018 and inform the researcher via email;

- $\quad$ ask any questions about the study at any time;

- receive a copy of the transcript of your interview recording;

- read over and comment on the transcript of your interview; 
- agree on another name for me to use rather than your real name;

- be able to read a summary report of findings from this research by emailing the researcher to request a copy.

\section{If you have any questions or problems, who can you contact?}

If you have any questions, either now or in the future, please feel free to contact either:

\section{Student:}

Name: Jie Gao

University email address: Role: primary supervisor

Jie.Gao@vuw.ac.nz

\section{Supervisors:} School: Education

Name: Dr. Flaviu-Adrian Hodis Name: Dr. Carolyn Frances Tait Role: secondary supervisor

Phone: + 64-4-463-9550 Phone: +64-4-463-9590

flaviu.hodis@vuw.ac.nz

\section{Human Ethics Committee information}

If you have any concerns about the ethical conduct of the research you may contact the Victoria University HEC Convener: Associate Professor Susan Corbett. Email susan.corbett@vuw.ac.nz or telephone +64-4-463 5480. 


\section{Appendix E: Consent Form for Interview Participants}

TE WHARE WĀNANGA O TE ŪPOKO O TE IKA A MĀUI

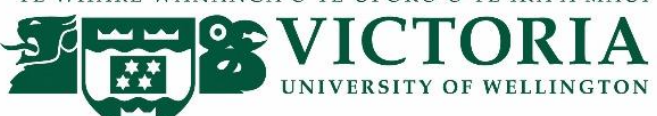

\section{CHINESE UNDERGRADUATE STUDENTS' MOTIVATION PROFILES: IDENTIFICATION AND SIGNIFICANCE CONSENT FOR INTERVIEW PARTICIPANTS}

This consent form will be held for seven years.

Researcher: Jie Gao, Faculty of Education, Victoria University of Wellington.

- I have read the Information Sheet and the project has been explained to me. My questions have been answered to my satisfaction. I understand that I can ask further questions at any time.

- I agree to take part in an audio recorded online interview.

I understand that:

- I may withdraw from this study at any point before January 15, 2018, without giving any reason, and any information that I have provided will be destroyed.

- The information I have provided will be destroyed seven years after the research is finished.

- Any information I provide will be kept confidential to the researcher and the supervisors. I understand that the results will be used for a $P h D$ thesis and a summary of the results may be used in academic reports, journal articles, book chapters, or presentations at conferences. 
- My name will not be used in reports, nor will any information that would identify me.

- I would like a copy of the transcript of my interview:

Yes $\square \quad$ No

- I would like a summary of my interview:

Yes $\square \quad$ No

- I would like to receive a summary report of findings from this

Yes $\square \quad$ No research and have added my email address below.

Signature of participant:

Name of participant:

Date:

Contact details:

Email:

QQ number or Wechat ID: 


\section{Appendix F: Samples of Interview Questions}

1. Tell me about your studying in this university?

- What is your major and why did you choose this major?

2. What do you do when you are required to write an essay or class report?

- Can you think of an example?

3. Tell me about a time when you found a learning task difficult.

- What was the difficulty or challenge?

- What did you do?

4. What do you do when you learn new knowledge in a class?

- Can you think of a specific example?

5. What do you do if there are parts of a course that are not interesting?

- Can you think of a specific example?

- What motivated you to work on it or what motivated you to give up and start to do something else? 